\title{
Light-Enabled Enantiodivergence: Stereospecific Reduction of Activated Alkenes using a Single Organocatalyst Enantiomer
}

Theresa Hostmann, John J. Molloy, Kathrin Bussmann and Ryan Gilmour* Organisch-Chemisches Institut, Westfälische Wilhelms-Universität Münster, Corrensstrasse 40, 48149 Münster, Germany

- General information 2

- Synthesis of alkenes 4

- Synthesis of E-nitrostyrenes 5

- Synthesis of Z-nitrostyrenes 9

- Synthesis of nitroalkanes 13

- Synthesis of thiourea catalysts 32

- Reaction optimization 35

- Synthesis of drug scaffolds 39

- References 49

- UV/Vis Absorption spectra 50

- NMR spectra of key compounds 51 


\section{General information}

All chemicals were purchased as reagent grade and used without further purification. Solvents for purification (extraction and column chromatography) were purchased as technical grade and distilled on the rotary evaporator prior to use. Solvents for analytical HPLC were purchased as HPLC grade (isopropanol), per analysis grade ( $n$-hexane, methanol, ethanol, dibutylamine). As stationary phase for column chromatography $\mathrm{SiO}_{2}-60$ (230-400 mesh ASTM; Fluka) was used. Analytical thin layer chromatography (TLC) was performed on glass plates pre-coated with $\mathrm{SiO}_{2}-$ 60 F254 (Merck) and visualized with a UV-lamp $(254 \mathrm{~nm})$ and $\mathrm{KMnO}_{4}$ solution or $\mathrm{I}_{2}$ on silica gel. Concentration in vacuo was performed at ca. 10 mbar and $50^{\circ} \mathrm{C}$, drying at ca. $10^{-2}$ mbar at room temperature. NMR spectra were measured by the NMR service of the OrganischChemisches Institut, Westfälische Wilhelms-Universität Münster on a Bruker Avance II 300, a Bruker Avance II 400, an Agilent DD2 500 or an Agilent DD2 600 spectrometer at room temperature. ${ }^{1} \mathrm{H}$-NMR spectra are reported as follows: chemical shift $\delta$ in ppm (multiplicity, coupling constant $J$ in $\mathrm{Hz}$, number of protons, assignment of proton). The deuterated solvent residual peak was used as internal reference: $\mathrm{CHCl}_{3}\left(\delta_{\mathrm{H}}=7.26 \mathrm{ppm}\right) .{ }^{13} \mathrm{C}-\mathrm{NMR}$ spectra are reported as follows: chemical shift $\delta$ in ppm (multiplicity if different from singlet, coupling constant $J$ in $\mathrm{Hz}$, assignment of carbon). The solvent residual peak was used as internal reference: $\mathrm{CDCl}_{3}\left(\delta_{\mathrm{C}}=77.16 \mathrm{ppm}\right) .{ }^{19} \mathrm{~F}-\mathrm{NMR}$ spectra are reported as follows: chemical shift $\delta$ in ppm (multiplicity, coupling constant $J$ in $\mathrm{Hz}$, assignment of fluorine [if more than one fluorine present]). The resonance multiplicity is abbreviated as: $s$ (singlet), $d$ (doublet), $t$ (triplet), $q$ (quartet), $\mathrm{m}$ (multiplet), sext (sextet) and br (broad). Assignments of unknown compounds are based on COSY $(\mathrm{HH}), \mathrm{HMBC}, \mathrm{HSQC}$ and NOESY spectra. Melting points were measured on a Büchi B-545 melting-point apparatus in open capillaries and are uncorrected. IR spectra were recorded on a Perkin-Elmer 100 FT-IR spectrometer, selected adsorption bands are reported in wavenumbers $\mathrm{v}\left(\mathrm{cm}^{-1}\right)$ and intensities are reported as: $\mathrm{w}$ (weak), $\mathrm{m}$ (medium) and $\mathrm{s}$ (strong). Mass spectra (HR-ESI, GC-EI) were measured by the MS service of the Organisch-Chemisches Institut, Westfälische Wilhelms-Universität Münster on a Bruker Daltonics MicrOTof ESI, a Thermo Fisher Scientific Orbitrap LTQ XL or a Thermo Fisher Scientific ISQ 7000 GC-MS. Isomerization reactions at $402 \mathrm{~nm}$ were performed with a set-up of 4 Winger WEPUV3-S2 UV Power LED Star (Schwarzlicht) $1.2 \mathrm{~W}$ (emission spectrum see Figure 1) . The forward current per chip was set to $700 \mathrm{~mA}$ with a resulting forward voltage of $3.4 \mathrm{~V}$ and a radiant flux of $1200 \mathrm{~mW}$. The distance between the reaction vessel and the UV-lamp was set to $1 \mathrm{~cm}$ (see Figure 2). Determination of the Z:E-ratio was done by integration of the ${ }^{1} \mathrm{H}-\mathrm{NMR}$ signal of the methyl group a to the aromatic ring. UV Absorption spectra were recorded on a Agilent Cary 60 $U V$-Vis Spectrophotometer. Optical rotations were identified with a JASCO P-200 polarimeter. Enantiomeric ratios were determined by analytical HPLC using the following chiral columns:

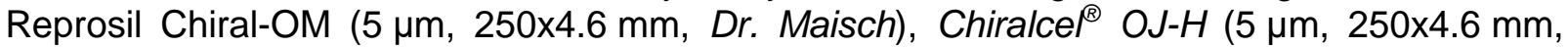
Daicel Chemical Industries, Ltd.), Reprosil Chiral-AM (10 $\mu \mathrm{m}, 250 \times 4.6 \mathrm{~mm}$, Dr. Maisch) or Chiralpac ${ }^{\circledR}$ IA (5 $\mu \mathrm{m}, 250 \times 4.6 \mathrm{~mm}$, Daicel Chemical Industries, Ltd.). 


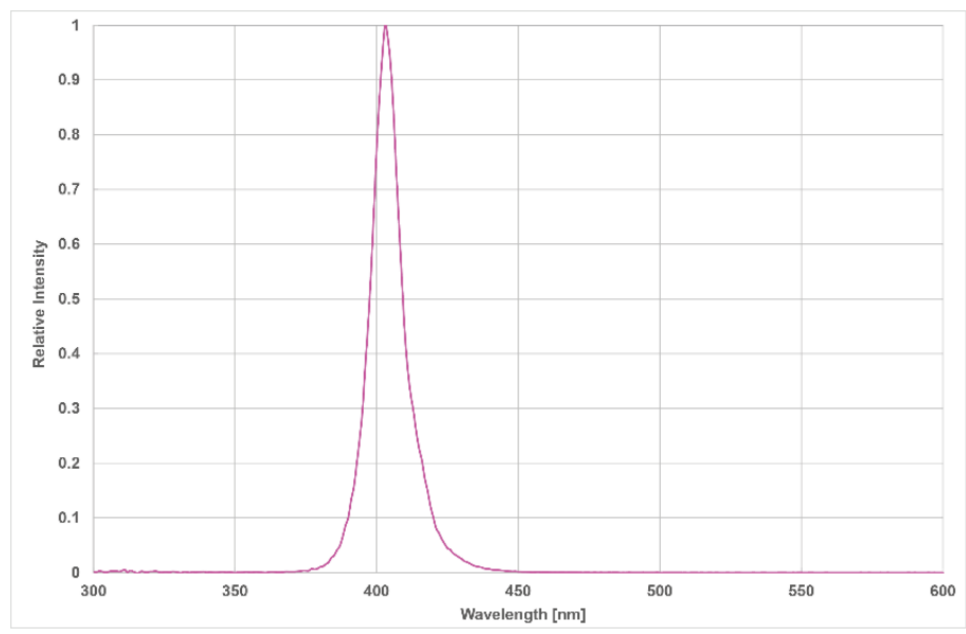

Figure 1: Emission spectrum of the utilized UV-lamp Winger WEPUV3-S2 UV Power LED Star (Schwarzlicht) 1.2 W.
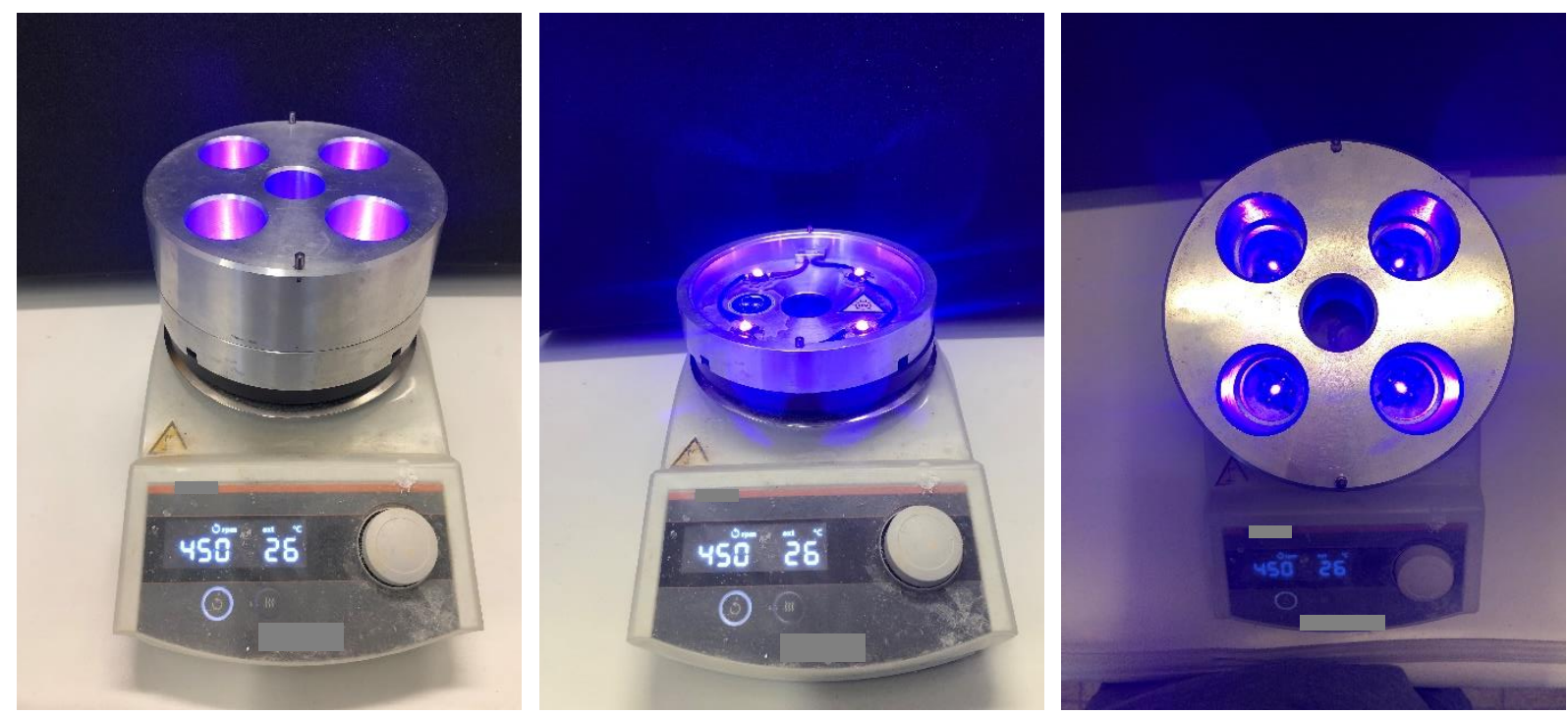

Figure 2: Reaction set-up for the isomerization of $E$-nitrostyrenes to $Z$-nitrostyrenes.

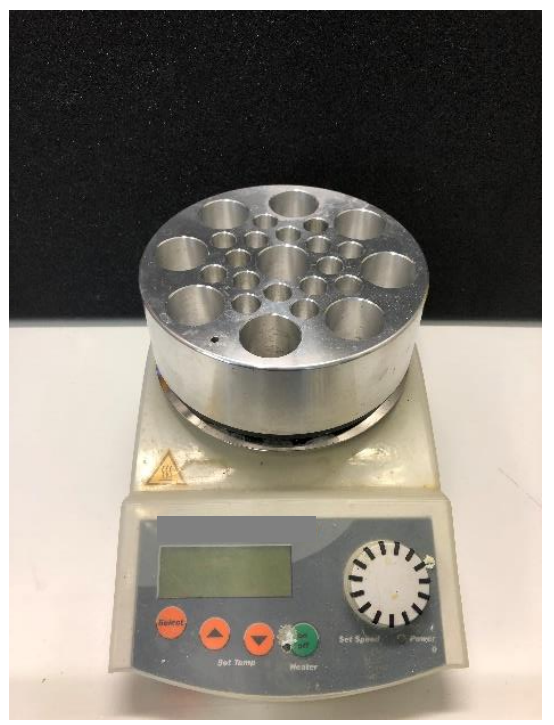

Figure 3: Reaction set up for the hydrogenation of nitrostyrenes to nitroalkanes. 


\section{Synthesis of alkenes}

\section{General procedure A for the preparation of terminal alkenes}

Following a modified literature procedure, ${ }^{[1]} \mathrm{PPh}_{3} \mathrm{MeBr}$ (2 eq.) was suspended in dry $\mathrm{Et}_{2} \mathrm{O}$ $(0.1 \mathrm{M})$ in a flame dried Schlenk flask under argon and cooled to $0{ }^{\circ} \mathrm{C}$ before $\mathrm{KO}$ tBu (2 eq.) was added. The resulting yellow suspension was stirred at $0^{\circ} \mathrm{C}$ for $1 \mathrm{~h}$ and the specified ketone (1 eq.) was added. While warming up to room temperature, the reaction mixture was monitored by TLC and upon conversion of the starting material or after stirring over night it was quenched with a sat. aq. $\mathrm{NH}_{4} \mathrm{Cl}$ solution. The separated organic layer was washed with water $(3 \mathrm{x})$, dried over $\mathrm{MgSO}_{4}$, filtered and concentrated under reduced pressure in a cold rotavap bath (ca. $20^{\circ} \mathrm{C},>200 \mathrm{mbar}$ ) due to the volatility of most of the terminal alkenes. Purification by column chromatography $\left(\mathrm{SiO}_{2}, n\right.$-pentane) afforded the terminal alkene.

\section{1-Methyl-2-(prop-1-en-2-yl)benzene (S1)}

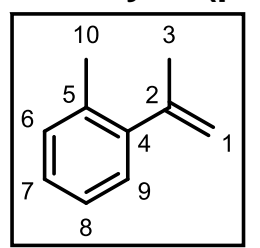

Prepared according to general procedure A, 2'-methylacetophenone $(2.6 \mathrm{~mL}$, $20 \mathrm{mmol})$ was converted to $\mathbf{S 1}$ in $4 \mathrm{~h}$, yielding a colorless liquid $(2.1 \mathrm{~g}$, $11.8 \mathrm{mmol}, 59 \%)$ after purification by column chromatography $\left(\mathrm{SiO}_{2}, n-\right.$ pentane).

$\mathbf{R}_{f}=0.79$ (n-pentane); ${ }^{1} \mathrm{H}-\mathrm{NMR}\left(400 \mathrm{MHz}, \mathrm{CDCl}_{3}\right): \delta=7.21-7.09(\mathrm{~m}, 4 \mathrm{H}, \mathrm{H} 6$, $\mathrm{H} 7, \mathrm{H} 8, \mathrm{H} 9$ ), $5.22-5.17$ ( $\mathrm{m}, 1 \mathrm{H}, \mathrm{H} 1$-cis), $4.88-4.82$ ( $\mathrm{m}, 1 \mathrm{H}, \mathrm{H} 1$-trans), 2.32 (s, 3H, H10), $2.07-2.02(\mathrm{~m}, 3 \mathrm{H}, \mathrm{H} 3) \mathrm{ppm} ;{ }^{13} \mathrm{C}\left\{{ }^{1} \mathrm{H}\right\}-\mathrm{NMR}\left(101 \mathrm{MHz}, \mathrm{CDCl}_{3}\right): \delta=146.0(\mathrm{C} 2)$, 144.0 (C4), 134.6 (C5), 130.2 (C6/7/8/9), 128.0 (C6/7/8/9), 126.9 (C6/7/8/9), 125.7 (C6/7/8/9), 114.8 (C1), 24.5 (C3), 19.9 (C10) ppm; IR (ATR): v = 3078 (w), 3018 (w), 2967 (w), 1639 (w), $1489(\mathrm{~m}), 1435$ (m), 1371 (w), 1303 (w), 1095 (w), 1048 (w), 1005 (w), 897 (s), 763 (s), 729 (s) $\mathrm{cm}^{-1}$; GC-El-MS: $\mathrm{m} / \mathrm{z}$ : [M] ${ }^{+}$calcd. for $\mathrm{C}_{10} \mathrm{H}_{12}{ }^{+}$: 132.10; found: 132.14 . The analytical data are in agreement with the literature. ${ }^{[2]}$

\section{(1-Cyclopropylvinyl)benzene (S2)}

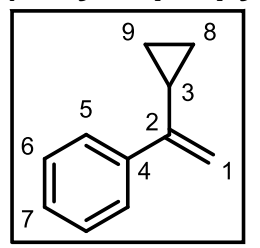

Prepared according to general procedure A, cyclopropyl(phenyl)methanone $(4.2 \mathrm{~mL}, 30 \mathrm{mmol})$ was converted to $\mathbf{S} 2$ over night, yielding a colorless liquid $(3.96 \mathrm{~g}, 27.4 \mathrm{mmol}, 92 \%)$ after purification by column chromatography $\left(\mathrm{SiO}_{2}\right.$, $n$-pentane).

$\mathbf{R}_{\boldsymbol{f}}=0.76$ ( $n$-pentane); ${ }^{1} \mathrm{H}-\mathrm{NMR}\left(600 \mathrm{MHz}, \mathrm{CDCl}_{3}\right): \delta=7.65-7.61(\mathrm{~m}, 2 \mathrm{H}, \mathrm{H} 5)$, $7.39-7.34(\mathrm{~m}, 2 \mathrm{H}, \mathrm{H} 6), 7.33-7.28(\mathrm{~m}, 1 \mathrm{H}, \mathrm{H} 7), 5.30(\mathrm{~m}, 1 \mathrm{H}, \mathrm{H} 1$-trans), 4.96 (m, 1H, H1-cis), $1.72-1.64(\mathrm{~m}, 1 \mathrm{H}, \mathrm{H} 3), 0.89-0.84(\mathrm{~m}, 2 \mathrm{H}, \mathrm{H} 8 / 9), 0.66-0.59(\mathrm{~m}, 2 \mathrm{H}$, $\mathrm{H} 8 / 9)$ ppm; ${ }^{13} \mathrm{C}\left\{{ }^{1} \mathrm{H}\right\}-N M R\left(151 \mathrm{MHz}, \mathrm{CDCl}_{3}\right): \delta=149.5(\mathrm{C} 2), 141.8(\mathrm{C} 4), 128.3(\mathrm{C} 6), 127.6(\mathrm{C} 7)$, 126.3 (C5), 109.1 (C1), 15.8 (C3), 6.82 (C8, C9) ppm; IR (ATR): v = 3083 (w), 3057 (w), 3004 (w), $2927(w), 1624(w), 1601(w), 1574(w), 1495(m), 1445(w), 1427(w), 1382(w), 1288(w)$, $1261(\mathrm{w}), 1173(\mathrm{w}), 1072(\mathrm{w}), 1051(\mathrm{w}), 1021(\mathrm{~m}), 939(\mathrm{~m}), 889(\mathrm{~s}), 837(\mathrm{w}), 820(\mathrm{~m}), 773(\mathrm{~s})$, 700 (s) $\mathrm{cm}^{-1}$; GC-EI-MS: $\mathrm{m} / \mathrm{z}$ : [M] ${ }^{+}$calcd. for $\mathrm{C}_{11} \mathrm{H}_{12}{ }^{+}: 144.10$; found: 144.13 . The analytical data are in agreement with the literature. ${ }^{[3]}$ 


\section{Synthesis of E-nitrostyrenes}

\section{General procedure B for the preparation of the $(E)$-nitrostyrenes}

Following a modified literature procedure, ${ }^{[1]} \mathrm{Ce}\left(\mathrm{NH}_{4}\right)_{2}\left(\mathrm{NO}_{3}\right)_{6}$ (1 eq.) and $\mathrm{NaNO}_{2}$ (10 eq.) were suspended in $\mathrm{CHCl}_{3}(0.08 \mathrm{M})$. Subsequently the specified terminal alkene (1 eq.) and $\mathrm{AcOH}$ (12 eq.) were added. The flask was connected to a bubbler containing a sat. aq. $\mathrm{NaHCO}_{3}$ solution and sonicated while the sonicator bath slowly heated itself up to ca. $50^{\circ} \mathrm{C}$. The reaction was monitored by TLC and upon conversion of the starting material the remaining solids were filtered off. The filtrate was quenched with a sat. aq. $\mathrm{NaHCO}_{3}$ solution and washed two additional times with $\mathrm{NaHCO}_{3}$ and brine. The separated organic layer was dried over $\mathrm{MgSO}_{4}$, filtered and concentrated in vacuo. Purification by column chromatography $\left(\mathrm{SiO}_{2}, \mathrm{Et}_{2} \mathrm{O}: n\right.$-pentane) afforded the $(E)$-nitrostyrene.

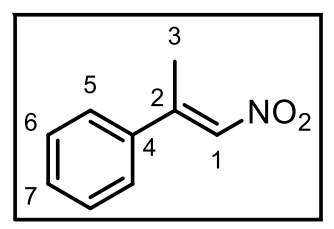

\section{(E)-(1-Nitroprop-1-en-2-yl)benzene ( $E$-1)}

Prepared according to general procedure $\mathbf{B}$, a-methyl styrene $(3.9 \mathrm{~mL}$, $30 \mathrm{mmol})$ was converted to $E-1$ in $5 \mathrm{~h}$, yielding a yellow oil $(3.2 \mathrm{~g}$, $19.6 \mathrm{mmol}, 65 \%)$ after purification by column chromatography $\left(\mathrm{SiO}_{2}\right.$, $\mathrm{Et}_{2} \mathrm{O}: n$-pentane, $\left.5: 95\right)$.

$\mathbf{R}_{\boldsymbol{f}}=0.58\left(\mathrm{Et}_{2} \mathrm{O}: n\right.$-pentane, 5:95); ${ }^{1} \mathrm{H}-\mathrm{NMR}\left(600 \mathrm{MHz}, \mathrm{CDCl}_{3}\right): \delta=7.47-$ $7.43(\mathrm{~m}, 5 \mathrm{H}, \mathrm{H} 5, \mathrm{H} 6, \mathrm{H} 7), 7.31\left(\mathrm{q},{ }^{4} J_{\mathrm{HH}}=1.4 \mathrm{~Hz}, 1 \mathrm{H}, \mathrm{H} 1\right), 2.65\left(\mathrm{~d},{ }^{4} J_{\mathrm{HH}}=\right.$ $1.4 \mathrm{~Hz}, 3 \mathrm{H}, \mathrm{H} 3) \mathrm{ppm} ;{ }^{13} \mathrm{C}\left\{{ }^{1} \mathrm{H}\right\}-\mathrm{NMR}\left(101 \mathrm{MHz}, \mathrm{CDCl}_{3}\right): \delta=150.1$ (C2), 138.4 (C4), 136.5 (C1), 130.5 (C7), 129.2 (C5/6), 127.0 (C5/6), 18.7 (C3) ppm; IR (ATR): $\tilde{v}=3105$ (w), 3060 (w), 2841 $(\mathrm{w}), 1622(\mathrm{~m}), 1575(\mathrm{w}), 1508(\mathrm{~s}), 1444(\mathrm{~m}), 1374(\mathrm{~m}), 1334(\mathrm{~s}), 1255(\mathrm{~m}), 1186(\mathrm{w}), 1160(\mathrm{w})$, 1078 (w), 1028 (w), 1000 (w), $919(\mathrm{~m}), 833(\mathrm{~m}), 804$ (w), 765 (s), 737 (s), 639 (s) cm-1; GC-EI-MS: $m / z$ : [M] ${ }^{+}$calcd. for $\mathrm{C}_{9} \mathrm{H}_{9} \mathrm{NO}_{2}{ }^{+}:$163.10; found: 163.11 . The analytical data are in agreement with the literature. ${ }^{[1]}$

\section{(E)-1-Methyl-4-(1-nitroprop-1-en-2-yl)benzene (E-2)}

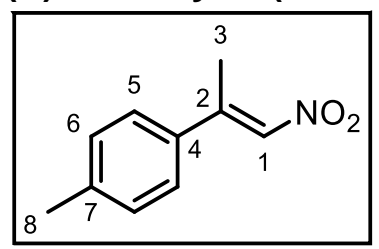

Prepared according to general procedure B, 1-methyl-4-(prop-1-en-2yl)benzene $(2.6 \mathrm{~g}, 20 \mathrm{mmol})$ was converted to $E-2$ in $5 \mathrm{~h}$, yielding a yellow oil $(1.5 \mathrm{~g}, 8.5 \mathrm{mmol}, 42 \%)$ after purification by column chromatography $\left(\mathrm{SiO}_{2}, \mathrm{Et}_{2} \mathrm{O}: n\right.$-pentane, 5:95).

$\mathbf{R}_{\boldsymbol{f}}=0.82\left(\mathrm{Et}_{2} \mathrm{O}: n\right.$-pentane, 5:95); ${ }^{1} \mathrm{H}-\mathrm{NMR}\left(600 \mathrm{MHz}, \mathrm{CDCl}_{3}\right): \delta=7.39$ $-7.33(\mathrm{~m}, 2 \mathrm{H}, \mathrm{H} 5), 7.32\left(\mathrm{q},{ }^{4} \mathrm{~J}_{\mathrm{HH}}=1.4 \mathrm{~Hz}, 1 \mathrm{H}, \mathrm{H} 1\right), 7.26-7.23(\mathrm{~m}, 2 \mathrm{H}$, $\mathrm{H} 6), 2.64\left(\mathrm{~d},{ }^{4} \mathrm{~J}_{\mathrm{HH}}=1.4 \mathrm{~Hz}, 3 \mathrm{H}, \mathrm{H} 3\right), 2.40(\mathrm{~s}, 3 \mathrm{H}, \mathrm{H} 8) \mathrm{ppm} ;{ }^{13} \mathrm{C}\left\{{ }^{1} \mathrm{H}\right\}-\mathrm{NMR}\left(151 \mathrm{MHz}, \mathrm{CDCl}_{3}\right)$ : $\delta=150.1$ (C2), 141.0 (C7), 135.9 (C1), 135.5 (C4), 129.9 (C6), 126.9 (C5), 21.4 (C8), 18.6 (C3) ppm; IR (ATR): $\tilde{v}=3678(w), 3107(w), 2924(w), 1618(\mathrm{~m}), 1607(\mathrm{~m}), 1568(\mathrm{w}), 1506(\mathrm{~s})$, 1441 (w), 1410 (w), $1374(w), 1332$ (s), $1258(\mathrm{~m}), 1216(\mathrm{w}), 1190(\mathrm{w}), 1126(\mathrm{w}), 1077(\mathrm{w}), 1038$ (w), 1018 (w), 920 (m), 836 (w), 811 (s), 709 (s) $\mathrm{cm}^{-1}$; GC-EI-MS: m/z: [M] ${ }^{+}$calcd. for $\mathrm{C}_{10} \mathrm{H}_{11} \mathrm{NO}_{2}{ }^{+}: 177.10$; found: 177.12 . The analytical data are in agreement with the literature. ${ }^{[4]}$

\section{(E)-1-Methoxy-4-(1-nitroprop-1-en-2-yl)benzene (E-3)}

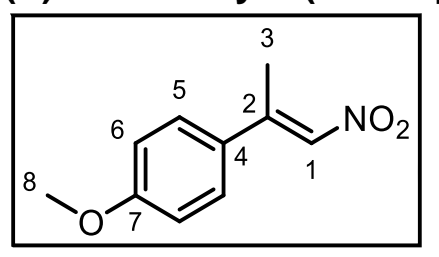

Prepared according to general procedure B, 1-methoxy-4-(prop-1en-2-yl)benzene $(2.96 \mathrm{~g}, 20 \mathrm{mmol})$ was converted to $E-3$ in $4 \mathrm{~h}$, yielding a yellow solid (936 $\mathrm{mg}, 4.85 \mathrm{mmol}, 24 \%$ ) after purification by column chromatography $\left(\mathrm{SiO}_{2}, \mathrm{Et}_{2} \mathrm{O}: n\right.$-pentane, 5:95).

$\mathbf{R}_{\boldsymbol{f}}=0.24\left(\mathrm{Et}_{2} \mathrm{O}: n\right.$-pentane, 5:95); ${ }^{1} \mathrm{H}-\mathrm{NMR}\left(600 \mathrm{MHz}, \mathrm{CDCl}_{3}\right)$ : $\delta=7.45-7.42(\mathrm{~m}, 2 \mathrm{H}, \mathrm{H} 5), 7.34\left(\mathrm{q},{ }^{4} \mathrm{~J}_{\mathrm{HH}}=1.4 \mathrm{~Hz}, 1 \mathrm{H}, \mathrm{H} 1\right), 6.97-$ $6.93(\mathrm{~m}, 2 \mathrm{H}, \mathrm{H} 6), 3.86(\mathrm{~s}, 3 \mathrm{H}, \mathrm{H} 8), 2.64\left(\mathrm{~d},{ }^{4} \mathrm{~J}_{\mathrm{HH}}=1.4 \mathrm{~Hz}, 3 \mathrm{H}, \mathrm{H} 3\right) \mathrm{ppm} ;{ }^{13} \mathbf{C}\left\{{ }^{1} \mathrm{H}\right\}-\mathbf{N M R}(151 \mathrm{MHz}$, $\left.\mathrm{CDCl}_{3}\right): \delta=161.8(\mathrm{C} 2), 149.8(\mathrm{C} 7), 135.3(\mathrm{C} 1), 130.4(\mathrm{C} 4), 128.6(\mathrm{C} 5), 114.6(\mathrm{C} 6), 55.6(\mathrm{C} 8)$, 18.5 (C3) ppm; IR (ATR): v = 3105 (w), 3012 (w), 2937 (w), 2840 (w), 1598 (s), 1571 (w), 1556 (w), 1505 (s), 1462 (m), 1441 (w), 1418 (w), 1374 (w), 1328 (s), 1291 (s), 1245 (s), 1179 (s), 1121 (w), 1078 (w), 1026 (s), $920(\mathrm{~m}), 823$ (s), $784(\mathrm{~m}), 734$ (w), 710 (w) cm-1; GC-EI-MS: $\mathrm{m} / \mathrm{z}$ : [M] ${ }^{+}$calcd. for $\mathrm{C}_{10} \mathrm{H}_{11} \mathrm{NO}_{3}{ }^{+}: 193.07334$; found: 193.07328 ; m.p.: $33-34{ }^{\circ} \mathrm{C}$. The analytical data are in agreement with the literature. ${ }^{[4]}$ 

yl)benzene $(3.23 \mathrm{~g}, 23.7 \mathrm{mmol})$ was converted to $E-4$ in $4 \mathrm{~h}$, yielding a yellow oil $(2.49 \mathrm{~g}, 13.8 \mathrm{mmol}, 58 \%)$ after purification by column chromatography $\left(\mathrm{SiO}_{2}, \mathrm{Et}_{2} \mathrm{O}: n\right.$-pentane, 5:95).

$\mathbf{R}_{f}=0.46\left(\mathrm{Et}_{2} \mathrm{O}: n\right.$-pentane, $\left.5: 95\right) ;{ }^{1} \mathrm{H}-\mathrm{NMR}\left(600 \mathrm{MHz}, \mathrm{CDCl}_{3}\right): \delta=7.47-$ $7.43(\mathrm{~m}, 2 \mathrm{H}, \mathrm{H} 5), 7.28\left(\mathrm{q},{ }^{4} \mathrm{JHH}_{\mathrm{HH}}=1.4 \mathrm{~Hz}, 1 \mathrm{H}, \mathrm{H} 1\right), 7.15-7.11(\mathrm{~m}, 2 \mathrm{H}$, $\mathrm{H6}), 2.63\left(\mathrm{~d},{ }^{4} \mathrm{~J}_{\mathrm{HH}}=1.4 \mathrm{~Hz}, 3 \mathrm{H}, \mathrm{H} 3\right) \mathrm{ppm} ;{ }^{13} \mathrm{C}\left\{{ }^{1} \mathrm{H}\right\}-\mathbf{N M R}\left(151 \mathrm{MHz}, \mathrm{CDCl}_{3}\right): \delta=164.1\left(\mathrm{~d},{ }^{1} J_{\mathrm{CF}}=\right.$ $251.6 \mathrm{~Hz}, \mathrm{C} 7), 148.8(\mathrm{C} 2), 136.4(\mathrm{C} 1), 134.4\left(\mathrm{~d},{ }^{4} J_{\mathrm{CF}}=3.4 \mathrm{~Hz}, \mathrm{C} 4\right), 129.0$ (d, $\left.{ }^{3} \mathrm{~J}_{\mathrm{CF}}=8.5 \mathrm{~Hz}, \mathrm{C} 5\right)$, $116.4\left(\mathrm{~d},{ }^{2} J_{\mathrm{CF}}=21.9 \mathrm{~Hz}, \mathrm{C} 6\right), 18.73(\mathrm{C} 3) \mathrm{ppm} ;{ }^{19} \mathrm{~F}-\mathrm{NMR}(564 \mathrm{MHz}):-109.8$ (tt, ${ }^{3} J_{\mathrm{FH}}=8.3 \mathrm{~Hz}$, $\left.{ }^{4} J_{\mathrm{FH}}=5.1 \mathrm{~Hz}\right) \mathrm{ppm}$; IR (ATR): $\tilde{v}=3108(\mathrm{w}), 2967(\mathrm{w}), 2849(\mathrm{w}), 1621(\mathrm{~m}), 1601(\mathrm{~m}), 1505(\mathrm{~s})$, 1437 (w), 1409 (w), 1375 (w), 1335 (s), 1283 (s), 1233 (s), 1163 (s), 1104 (w), 1077 (w), 1014 (w), 920 (m), 825 (s), 791 (m), 729 (w), $712(w) \mathrm{cm}^{-1}$; GC-El-MS: m/z: [M] ${ }^{+}$calcd. for $\mathrm{C}_{9} \mathrm{H}_{8} \mathrm{FNO}_{2}{ }^{+}$: 181.10; found: 181.10 . The analytical data are in agreement with the literature. ${ }^{[4]}$

\section{(E)-1-Chloro-4-(1-nitroprop-1-en-2-yl)benzene (E-5)}

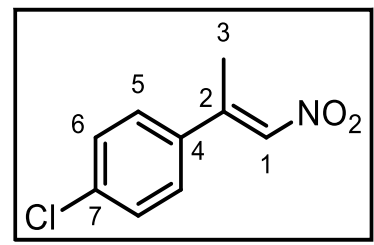

Prepared according to general procedure B, 1-chloro-4-(prop-1-en-2yl)benzene $(3.05 \mathrm{~g}, 20 \mathrm{mmol})$ was converted to $E-5$ in $4 \mathrm{~h}$, yielding a yellow oil $(1.88 \mathrm{~g}, 9.5 \mathrm{mmol}, 48 \%)$ after purification by column chromatography $\left(\mathrm{SiO}_{2}, \mathrm{Et}_{2} \mathrm{O}: n\right.$-pentane, 5:95).

$\mathbf{R}_{\boldsymbol{f}}=0.46\left(\mathrm{Et}_{2} \mathrm{O}: n\right.$-pentane, $\left.5: 95\right) ;{ }^{1} \mathrm{H}-\mathrm{NMR}\left(600 \mathrm{MHz}, \mathrm{CDCl}_{3}\right): \delta=7.45$ $-7.36(\mathrm{~m}, 4 \mathrm{H}, \mathrm{H} 5, \mathrm{H} 6), 7.28\left(\mathrm{q},{ }^{4} \mathrm{~J}_{\mathrm{HH}}=1.4 \mathrm{~Hz}, 1 \mathrm{H}, \mathrm{H} 1\right), 2.62\left(\mathrm{~d},{ }^{4} \mathrm{~J}_{\mathrm{HH}}=\right.$ $1.4 \mathrm{~Hz}, 3 \mathrm{H}, \mathrm{H} 3) \mathrm{ppm} ;{ }^{13} \mathrm{C}\left\{{ }^{1} \mathrm{H}\right\}-\mathrm{NMR}\left(151 \mathrm{MHz}, \mathrm{CDCl}_{3}\right): \delta=148.6(\mathrm{C} 2), 136.8$ (C4, C7), 136.6 (C1), 129.7 (C5/6), 128.3 (C5/6), 18.6 (C3) ppm; IR (ATR): v = $3106(\mathrm{w}), 1621(\mathrm{~m}), 1591(\mathrm{~m})$, $1564(\mathrm{w}), 1510$ (s), $1489(\mathrm{~m}), 1436(\mathrm{w}), 1401(\mathrm{~m}), 1374(\mathrm{w}), 1331(\mathrm{~s}), 1254(\mathrm{w}), 1185(\mathrm{w}), 1095$ (s), 1011 (s), 920 (w), 819 (s), 754 (s), 722 (w), 662 (w) cm ${ }^{-1}$; GC-EI-MS: m/z: [M] ${ }^{+}$calcd. for $\mathrm{C}_{9} \mathrm{H}_{8} \mathrm{CINO}_{2}{ }^{+}$: 197.02; found: 197.08 . The analytical data are in agreement with the literature. ${ }^{[4]}$

\section{(E)-1-(1-Nitroprop-1-en-2-yl)-4-(trifluoromethyl)benzene (E-6)}

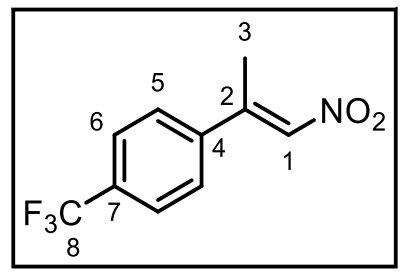

Prepared according to general procedure B, 1-(prop-1-1en-2-yl)-4(trifluoromethyl)benzene $(2.95 \mathrm{~g}, 20 \mathrm{mmol})$ was converted to $E-6$ in $5 \mathrm{~h}$, yielding a yellow oil $(1.34 \mathrm{~g}, 5.8 \mathrm{mmol}, 37 \%)$ after purification by column chromatography $\left(\mathrm{SiO}_{2}, \mathrm{Et}_{2} \mathrm{O}: n\right.$-pentane, $\left.5: 95\right)$.

$\mathbf{R}_{\boldsymbol{f}}=0.43$ (Et $\mathrm{E}_{2} \mathrm{O}: n$-pentane, 5:95); ${ }^{1} \mathrm{H}-\mathrm{NMR}\left(600 \mathrm{MHz}, \mathrm{CDCl}_{3}\right)$ : $\delta=7.71\left(\mathrm{~d},{ }^{2}{ }_{\mathrm{HHH}}=8.3 \mathrm{~Hz}, 2 \mathrm{H}, \mathrm{H} 6\right), 7.56\left(\mathrm{~d},{ }^{2}{ }_{\mathrm{HH}}=8.3 \mathrm{~Hz}, 2 \mathrm{H}, \mathrm{H} 5\right)$, $7.29\left(\mathrm{~d},{ }^{4} J_{\mathrm{HH}}=1.4 \mathrm{~Hz}, 1 \mathrm{H}, \mathrm{H} 1\right), 2.65\left(\mathrm{~d},{ }^{4} \mathrm{~J}_{\mathrm{HH}}=1.5 \mathrm{~Hz}, 3 \mathrm{H}, \mathrm{H} 3\right) \mathrm{ppm}$; ${ }^{13} \mathrm{C}\left\{{ }^{1} \mathrm{H}\right\}-N M R\left(151 \mathrm{MHz}, \mathrm{CDCl}_{3}\right): \delta=148.2(\mathrm{C} 2), 142.0\left(\mathrm{~d},{ }^{5} \mathrm{~J}_{\mathrm{CF}}=1.3 \mathrm{~Hz}, \mathrm{C} 4\right), 137.4$ (C1), 132.3 (d, $\left.{ }^{2} J_{\mathrm{CF}}=33.0 \mathrm{~Hz}, \mathrm{C7}\right), 127.4(\mathrm{C} 5), 126.2\left(\mathrm{q},{ }^{3} J_{\mathrm{CF}}=3.7 \mathrm{~Hz}, \mathrm{C6}\right), 123.8\left(\mathrm{~d},{ }^{1} J_{\mathrm{CF}}=272.3 \mathrm{~Hz}, \mathrm{C} 8\right)$, 18.7 (C3) ppm; ${ }^{19} \mathrm{~F}-\mathrm{NMR}\left(470 \mathrm{MHz}, \mathrm{CDCl}_{3}\right) \delta=-62.97$ (s) ppm; IR (ATR): $\tilde{v}=3107$ (w), 2961 $(w), 1625(w), 1574(w), 1518(m), 1437(w), 1409(w), 1377(w), 1346(m), 1319(s), 1257(w)$, $1167(\mathrm{~m}), 1115$ (s), $1082(\mathrm{~m}), 1063$ (s), 1015 (s), 959 (w), 919 (w), 829 (s), 777 (w), 727 (s) cm-1; GC-EI-MS: $\mathrm{m} / \mathrm{z}$ : [M-H] ${ }^{+}$calcd. for $\mathrm{C}_{10} \mathrm{H}_{7} \mathrm{NO}_{2} \mathrm{~F}_{3}{ }^{+}$: 230.04234; found: 230.04250 . The analytical data are in agreement with the literature..$^{[5]}$

\section{(E)-1-Methyl-2-(1-nitroprop-1-en-2-yl)benzene (E-7)}

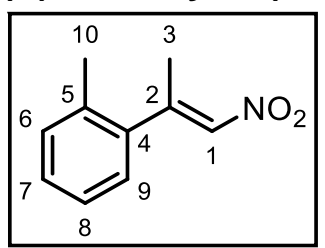

Prepared according to general procedure B, S1 $(2.0 \mathrm{~g}, 15.1 \mathrm{mmol})$ was converted to $E-7$ in $4 \mathrm{~h}$, yielding a yellow oil (557 mg, $3.14 \mathrm{mmol}, 21 \%$ ) after purification by column chromatography $\left(\mathrm{SiO}_{2}, \mathrm{Et}_{2} \mathrm{O}: n\right.$-pentane, 5:95). $\mathbf{R}_{\boldsymbol{f}}=0.53\left(\mathrm{Et}_{2} \mathrm{O}: n\right.$-pentane, $\left.5: 95\right) ;{ }^{1} \mathrm{H}-\mathrm{NMR}\left(500 \mathrm{MHz}, \mathrm{CDCl}_{3}\right): \delta=7.30$ (td, $\left.{ }^{3} J_{\mathrm{HH}}=7.4 \mathrm{~Hz},{ }^{4} J_{\mathrm{HH}}=1.4 \mathrm{~Hz}, 1 \mathrm{H}, \mathrm{H} 7\right), 7.26-7.20(\mathrm{~m}, 2 \mathrm{H}, \mathrm{H} 8, \mathrm{H} 6), 7.10$ $\left(\mathrm{dd},{ }^{3} J_{\mathrm{HH}}=7.6 \mathrm{~Hz},{ }^{4} J_{\mathrm{HH}}=1.4 \mathrm{~Hz}, 1 \mathrm{H}, \mathrm{H} 9\right), 7.00\left(\mathrm{q},{ }^{4} J_{\mathrm{HH}}=1.5 \mathrm{~Hz}, 1 \mathrm{H}, \mathrm{H} 1\right)$, $2.54\left(\mathrm{~d},{ }^{4} \mathrm{~J}_{\mathrm{HH}}=1.5 \mathrm{~Hz}, 3 \mathrm{H}, \mathrm{H} 3\right), 2.32(\mathrm{~s}, 3 \mathrm{H}, \mathrm{H} 10) \mathrm{ppm} ;{ }^{13} \mathrm{C}\left\{{ }^{1} \mathrm{H}\right\}-\mathrm{NMR}\left(126 \mathrm{MHz}, \mathrm{CDCl}_{3}\right)$ : $\delta=151.8$ (C2), 139.1 (C4), 137.7 (C1), 134.5 (C5), 131.0 (C8), 129.1 (C7), 127.4 (C9), 126.2 (C6), 21.0 (C3), 19.8 (C10) ppm; IR (ATR): $\tilde{v}=3100$ (w), 2962 (w), 1627 (w), 1513 (s), 1486 (m), 1455 (w), 1371 (w), 1341 (s), 1288 (w), 1248 (w), 1161 (w), 1123 (w), $1072(w), 1046(w)$, 
919 (w), $838(\mathrm{~m}), 788(\mathrm{w}), 761$ (s), 728 (s), $712(\mathrm{~m}) \mathrm{cm}^{-1}$; GC-EI-MS: $\mathrm{m} / \mathrm{z}$ : [M-H] ${ }^{+}$calcd. for $\mathrm{C}_{10} \mathrm{H}_{10} \mathrm{NO}_{2}{ }^{+}:$173.07061; found: 176.07063. The analytical data are in agreement with the literature. ${ }^{[5]}$

\section{(E)-1-Methyl-3-(1-nitroprop-1-en-2-yl)benzene (E-8)}

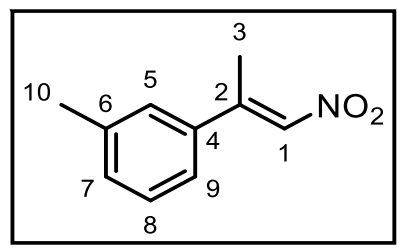

Prepared according to general procedure B, 1-methyl-3-(prop-1-en2-yl)benzene $(4.0 \mathrm{~g}, 30.3 \mathrm{mmol})$ was converted to $E-8$ in $4 \mathrm{~h}$, yielding a yellow oil $(2.52 \mathrm{~g}, 14.2 \mathrm{mmol}, 47 \%)$ after purification by column chromatography $\left(\mathrm{SiO}_{2}, \mathrm{Et}_{2} \mathrm{O}: n\right.$-pentane, 5:95).

$\mathbf{R}_{\boldsymbol{f}}=0.45\left(\mathrm{Et}_{2} \mathrm{O}: n\right.$-pentane, 5:95); ${ }^{1} \mathrm{H}-\mathrm{NMR}\left(500 \mathrm{MHz}, \mathrm{CDCl}_{3}\right)$ : $\delta=7.35-7.31(\mathrm{~m}, 1 \mathrm{H}, \mathrm{H} 5), 7.31\left(\mathrm{q},{ }^{4} \mathrm{~J}_{\mathrm{HH}}=1.5 \mathrm{~Hz}, 1 \mathrm{H}, \mathrm{H} 1\right), 7.28-$ $7.24(\mathrm{~m}, 3 \mathrm{H}, \mathrm{H} 7, \mathrm{H} 8, \mathrm{H} 9), 2.64\left(\mathrm{~d},{ }^{4} \mathrm{~J}_{\mathrm{HH}}=1.5 \mathrm{~Hz}, 3 \mathrm{H}, \mathrm{H} 3\right), 2.41(\mathrm{~s}, 3 \mathrm{H}, \mathrm{H} 10) \mathrm{ppm} ;{ }^{13} \mathrm{C}\left\{{ }^{1} \mathrm{H}\right\}-\mathrm{NMR}$ (126 MHz, $\mathrm{CDCl}_{3}$ ): $\delta=150.3$ (C2), 139.0 (C6), 138.5 (C4), 136.3 (C1), $131.3(\mathrm{C} 7 / 8 / 9), 129.1$ (C5), 127.6 (C7/8/9), 124.1 (C7/8/9), 21.5 (C10), 18.7 (C3) ppm; IR (ATR): v = 3107 (w), 3026 $(w), 2924(w), 1619(w), 1603(w), 1582(w), 1508(s), 1442(w), 1374(w), 1334(s), 1268(m)$, $1192(w), 1101(w), 1040(w), 999(w), 938$ (w), $882(w), 860(w), 833(\mathrm{~m}), 784(s), 715(\mathrm{~m}), 694$ (m) $\mathrm{cm}^{-1}$; GC-El-MS: $\mathrm{m} / \mathrm{z}$ : [M-H] ${ }^{+}$calcd. for $\mathrm{C}_{10} \mathrm{H}_{10} \mathrm{NO}_{2}{ }^{+}$: 176.07061; found: 176.07066. The analytical data are in agreement with the literature. ${ }^{[6]}$

\section{(E)-1-Chloro-3-(1-nitroprop-1-en-2-yl)benzene (E-9)}

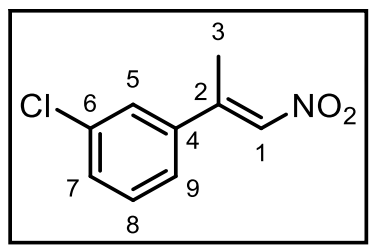

Prepared according to general procedure B, 1-chloro-3-(prop-1-en-2yl)benzene $(3.0 \mathrm{~g}, 15.2 \mathrm{mmol}$ ) was converted to $E-9$ in $5 \mathrm{~h}$, yielding a yellow oil $(2.07 \mathrm{~g}, 10.5 \mathrm{mmol}, 69 \%)$ after purification by column chromatography $\left(\mathrm{SiO}_{2}, \mathrm{Et}_{2} \mathrm{O}, n\right.$-pentane, 5:95).

$\mathbf{R}_{\boldsymbol{f}}=0.39\left(\mathrm{Et}_{2} \mathrm{O}: n\right.$-pentane, $\left.5: 95\right) ;{ }^{1} \mathrm{H}-\mathrm{NMR}\left(400 \mathrm{MHz}, \mathrm{CDCl}_{3}\right): \delta=7.47$ $-7.40(\mathrm{~m}, 2 \mathrm{H}, \mathrm{H} 5, \mathrm{H} 7), 7.42-7.34(\mathrm{~m}, 1 \mathrm{H}, \mathrm{H} 8), 7.33\left(\mathrm{dt},{ }^{3} \mathrm{~J}_{\mathrm{HH}}=7.6 \mathrm{~Hz}\right.$, $\left.{ }^{4} J_{\mathrm{HH}}=1.6 \mathrm{~Hz}, 1 \mathrm{H}, \mathrm{H} 9\right), 7.28\left(\mathrm{q},{ }^{4} \mathrm{~J}_{\mathrm{HH}}=1.4 \mathrm{~Hz}, 1 \mathrm{H}, \mathrm{H} 1\right), 2.62\left(\mathrm{~d},{ }^{4} J_{\mathrm{HH}}=1.4 \mathrm{~Hz}, 3 \mathrm{H}, \mathrm{H} 3\right) \mathrm{ppm}$; ${ }^{13} \mathrm{C}\left\{{ }^{1} \mathrm{H}\right\}-N M R\left(101 \mathrm{MHz}, \mathrm{CDCl}_{3}\right): \delta=148.4(\mathrm{C} 2), 140.2$ (C4), 137.0 (C1), 135.3 (C6), 130.5 (C7, C8), 127.2 (C5), 125.1 (C9), 18.6 (C3) ppm; IR (ATR): v = 3110 (w), 3080 (w) 3060 (w), 1619 (w), $1563(w), 1504(m), 1473(w), 1408(w), 1377(w), 1336(s), 1267(w), 1248(m), 1171(w)$, 1102 (w), 1082 (w), 1017 (w), 996 (w), 929 (w), 900 (w), 839 (m), 796 (s), 767 (w), 720 (w), 682 (s) $\mathrm{cm}^{-1}$; GC-El-MS: $\mathrm{m} / \mathrm{z}$ : [M] ${ }^{+}$calcd. for $\mathrm{C}_{9} \mathrm{H}_{8} \mathrm{CINO}_{2}{ }^{+}$: 197.02; found: 197.07. The analytical data are in agreement with the literature. ${ }^{[7]}$

\section{(E)-(1-Cyclopropyl-2-nitrovinyl)benzene (E-10)}

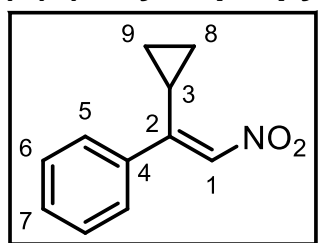

Prepared according to general procedure B, S2 $(2.0 \mathrm{~g}, 15.1 \mathrm{mmol})$ was converted to $E-10$ in $5 \mathrm{~h}$, yielding a yellow oil (557 mg, $3.14 \mathrm{mmol}, 21 \%$ ) after purification by column chromatography $\left(\mathrm{SiO}_{2}, \mathrm{Et}_{2} \mathrm{O}: n\right.$-pentane, 5:95). $\mathbf{R}_{\boldsymbol{f}}=0.45\left(\mathrm{Et}_{2} \mathrm{O}: n\right.$-pentane, 5:95); ${ }^{1} \mathrm{H}-\mathrm{NMR}\left(400 \mathrm{MHz}, \mathrm{CDCl}_{3}\right): \delta=7.40-$ $7.35(\mathrm{~m}, 3 \mathrm{H}, \mathrm{H} 6, \mathrm{H} 7), 7.20-7.16(\mathrm{~m}, 2 \mathrm{H}, \mathrm{H} 5), 7.07\left(\mathrm{~d},{ }^{4} \mathrm{~J}_{\mathrm{HH}}=0.8 \mathrm{~Hz}, 1 \mathrm{H}\right.$, $\mathrm{H} 1), 3.11\left(\mathrm{ttd},{ }^{3} \mathrm{~J}_{\mathrm{HH}}=8.4,5.3 \mathrm{~Hz},{ }^{4} \mathrm{~J}_{\mathrm{HH}}=0.9 \mathrm{~Hz}, 1 \mathrm{H}, \mathrm{H} 3\right), 1.09-1.04(\mathrm{~m}$, $2 \mathrm{H}, \mathrm{H} 8 / 9), 0.62-0.58(\mathrm{~m}, 2 \mathrm{H}, \mathrm{H} 8 / 9) \mathrm{ppm} ;{ }^{13} \mathrm{C}\left\{{ }^{1} \mathrm{H}\right\}-N M R\left(101 \mathrm{MHz}, \mathrm{CDCl}_{3}\right): \delta=157.2(\mathrm{C} 2)$, 137.5 (C1), 134.3 (C4), 129.3 (C7), 128.5 (C5), 128.4 (C6), 13.5 (C3), 8.2 (C8, C9) ppm; IR $($ ATR): $\tilde{v}=3100(w), 3016(w), 2928(w), 2854(w), 1597(\mathrm{~m}), 1575(w), 1552(w), 1509(s), 1489$ $(\mathrm{m}), 1443(\mathrm{~m}), 1427(\mathrm{w}), 1365(\mathrm{~m}), 1335(\mathrm{~s}), 1279(\mathrm{w}), 1258(\mathrm{w}), 1195(\mathrm{w}), 1178(\mathrm{w}), 1159(\mathrm{w})$, $1123(w), 1103(w), 1075(w), 1061(w), 1032(w), 1001(w), 920(m), 824(m), 772(s), 729(s)$, 701 (s), 667 (m) cm${ }^{-1}$; GC-El-MS: $m / z$ : $[\mathrm{M}-\mathrm{H}]^{+}$calcd. for $\mathrm{C}_{11} \mathrm{H}_{11} \mathrm{NO}_{2}{ }^{+}$: 189.0789; found: 188.07063 .

\section{(E)-1-Fluoro-2-(2-nitrovinyl)benzene (E-12)}

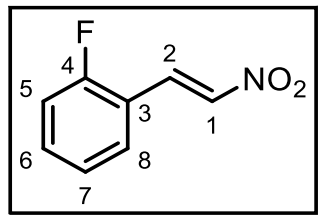

Following a modified literature procedure, ${ }^{[8]}$ a solution of 2fluorobenzaldehyde $\left(1.24 \mathrm{~g}, 10 \mathrm{mmol}\right.$, 1 eq.) in $\mathrm{MeNO}_{2}(0.65 \mathrm{~mL}$, $12 \mathrm{mmol}, 1.2$ eq.) and $\mathrm{MeOH}(16 \mathrm{~mL})$ was added at ca. $5^{\circ} \mathrm{C}$ to a solution of $\mathrm{NaOH}\left(2.5 \mathrm{M}\right.$ in $\mathrm{H}_{2} \mathrm{O}, 4.8 \mathrm{~mL}, 12 \mathrm{mmol}, 1.2$ eq.) and $\mathrm{MeOH}(4.8 \mathrm{~mL})$ over $30 \mathrm{~min}$. The reaction mixture was stirred for $30 \mathrm{~min}$, before a solution of $\mathrm{ZnCl}_{2}$ (6.82 g, $50 \mathrm{mmol}, 5$ eq.), aq. $\mathrm{HCl}$ (12 M, $2.1 \mathrm{~mL}, 25 \mathrm{mmol}, 2.5$ eq.) 
and $\mathrm{H}_{2} \mathrm{O}(2.7 \mathrm{~mL})$ was added at ca. $5^{\circ} \mathrm{C}$ over $30 \mathrm{~min}$. While warming up to room temperature, stirring was continued for further $3 \mathrm{~h}$. The light yellow precipitate was collected by filtration and washed with a solution of $\mathrm{MeOH}$ in $\mathrm{H}_{2} \mathrm{O}(40 \%, 3 \times 5 \mathrm{~mL})$ to yield $E-11$ as a light yellow solid (763 mg, $4.56 \mathrm{mmol}, 46 \%$ ).

${ }^{1} \mathrm{H}-\mathrm{NMR}\left(500 \mathrm{MHz}, \mathrm{CDCl}_{3}\right): \delta=8.06\left(\mathrm{~d},{ }^{3} \mathrm{~J}_{\mathrm{HH}}=13.8 \mathrm{~Hz}, 1 \mathrm{H}, \mathrm{H} 1\right), 7.73\left(\mathrm{~d},{ }^{3} \mathrm{~J}_{\mathrm{HH}}=13.8 \mathrm{~Hz}, 1 \mathrm{H}\right.$, $\mathrm{H} 2), 7.54-7.46(\mathrm{~m}, 2 \mathrm{H}, \mathrm{H} 8, \mathrm{H} 6), 7.27-7.16(\mathrm{~m}, 2 \mathrm{H}, \mathrm{H} 7, \mathrm{H} 5) \mathrm{ppm} ;{ }^{13} \mathbf{C}\left\{{ }^{1} \mathrm{H}\right\}-\mathrm{NMR}(126 \mathrm{MHz}$, $\left.\mathrm{CDCl}_{3}\right): \delta=162.0\left(\mathrm{~d},{ }^{1} J_{\mathrm{CF}}=256.4 \mathrm{~Hz}, \mathrm{C} 4\right), 139.6\left(\mathrm{~d},{ }^{3} J_{\mathrm{CF}}=11.5 \mathrm{~Hz}, \mathrm{C} 2\right), 133.8\left(\mathrm{~d},{ }^{3} J_{\mathrm{CF}}=9.1 \mathrm{~Hz}\right.$, C6), $132.6\left(\mathrm{~d},{ }^{4} J_{\mathrm{CF}}=0.7 \mathrm{~Hz}, \mathrm{C} 1\right), 131.5\left(\mathrm{~d},{ }^{3} \mathrm{~J}_{\mathrm{CF}}=2.5 \mathrm{~Hz}, \mathrm{C} 8\right), 125.2\left(\mathrm{~d},{ }^{4} J_{\mathrm{CF}}=3.6 \mathrm{~Hz}, \mathrm{C} 7\right), 118.6$ (d, $\left.{ }^{2} J_{\mathrm{CF}}=12.0 \mathrm{~Hz}, \mathrm{C} 3\right), 116.8\left(\mathrm{~d},{ }^{2} J_{\mathrm{CF}}=21.7 \mathrm{~Hz}, \mathrm{C} 5\right) \mathrm{ppm} ;{ }^{19} \mathrm{~F}-\left\{{ }^{1} \mathrm{H}\right\}-\mathrm{NMR}\left(282 \mathrm{MHz}, \mathrm{CDCl}_{3}\right)$ : $\delta=-109.11$ ppm; IR (ATR): $\tilde{v}=3163$ (w), 3049 (w), 2841 (w), 1633 (w), 1611 (m), 1507 (s), $1493(\mathrm{~s}), 1450(\mathrm{~m}), 1381(\mathrm{w}), 1346(\mathrm{~s}), 1305(\mathrm{w}), 1287(\mathrm{w}), 1262(\mathrm{w}), 1248(\mathrm{w}), 1234(\mathrm{w}), 1215$ $(\mathrm{m}), 1203(\mathrm{~m}), 1189(\mathrm{w}), 1160(\mathrm{w}), 1106(\mathrm{w}), 1037(\mathrm{w}), 967(\mathrm{~s}), 889(\mathrm{w}), 865(\mathrm{w}), 842(\mathrm{~m}), 809$ (w), 780 (w), 755 (s), 732 (s) cm${ }^{-1}$; GC-El-MS: $\mathrm{m} / \mathrm{z}$ : [M] ${ }^{+}$calcd. for $\mathrm{C}_{8} \mathrm{H}_{6} \mathrm{FNO}_{2}{ }^{+}$: 167.04; found: $167.07 ;$ m.p. $=50-53^{\circ} \mathrm{C}$. The analytical data are in agreement with the literature. ${ }^{[9]}$ 


\section{Synthesis of Z-nitrostyrenes}

\section{General procedure $\mathrm{C}$ for the preparation of the (Z)-nitrostyrenes}

In a flame dried $10 \mathrm{~mL}$ round bottom flask, the $(E)$-nitrostyrene $(0.5 \mathrm{mmol}, 1$ eq. $)$ was dissolved in dry $\mathrm{MeCN}(0.5 \mathrm{~mL}, 1 \mathrm{M})$. The flask was sealed with a rubber septum and the mixture was degassed by bubbling argon through it with a needle for ca. 2 min. The flask was connected to a balloon filled with argon and placed in the shown irradiation set up (Figure 2). After irradiation at $402 \mathrm{~nm}$ for $7 \mathrm{~h}$, the solvent was removed under reduced pressure and the residue was filtered over a plug of $\mathrm{SiO}_{2}$. Subsequent elution of the products with $\mathrm{Et}_{2} \mathrm{O}: n$-pentane $1: 9$ and concentration in vacuo yielded the mixture of the $(Z)$ - and $(E)$-isomer. The yield was determined by mass recovery based on NMR purity and the $Z$ : $E$-ratio was determined by integration of the ${ }^{1} \mathrm{H}-\mathrm{NMR}$ signal of the methyl group a to the aromatic ring (for $\mathbf{Z - 1}-\mathbf{Z}-\mathbf{- 9}$ ), the $\mathrm{CH}$-proton of the cyclopropyl (for Z-10) or the methanylyliden protons (for Z-11 and Z-12). A second purification by column chromatography $\left(\mathrm{SiO}_{2}, \mathrm{Et}_{2} \mathrm{O}: n\right.$-pentane) yielded the pure $(Z)$-nitrostyrene. Reported yields and $Z: E$ stereoselectivities are an average of two duplicate reactions.

\section{(Z)-(1-Nitroprop-1-en-2-yl)benzene (Z-1)}

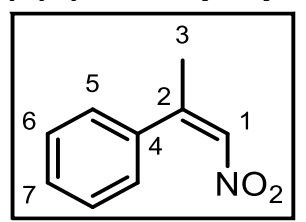

Prepared according to general procedure C, $\mathbf{E}-\mathbf{1}(82 \mathrm{mg})$ was converted to $Z-1$ yielding a yellow oil as a mixture of isomers $(58.8 \mathrm{mg}, 0.36 \mathrm{mmol}, 72 \%$, $Z: E=92: 08)$.

$\mathbf{R}_{\boldsymbol{f}}=0.42\left(\mathrm{Et}_{2} \mathrm{O}: n\right.$-pentane, 5:95); ${ }^{1} \mathrm{H}-\mathrm{NMR}\left(600 \mathrm{MHz}, \mathrm{CDCl}_{3}\right): \delta=7.43-$ $7.35(\mathrm{~m}, 3 \mathrm{H}, \mathrm{H} 6, \mathrm{H} 7), 7.20\left(\mathrm{dd},{ }^{3} \mathrm{~J}_{\mathrm{HH}}=7.7 \mathrm{~Hz},{ }^{4} J_{\mathrm{HH}}=1.8 \mathrm{~Hz}, 2 \mathrm{H}, \mathrm{H} 5\right), 7.06$ $\left(\mathrm{q},{ }^{4} J_{\mathrm{HH}}=1.4 \mathrm{~Hz}, 1 \mathrm{H}, \mathrm{H} 1\right), 2.19\left(\mathrm{~d},{ }^{4} J_{\mathrm{HH}}=1.4 \mathrm{~Hz}, 3 \mathrm{H}, \mathrm{H} 3\right) \mathrm{ppm}$; ${ }^{13} \mathrm{C}\left\{{ }^{1} \mathrm{H}\right\}-N M R\left(151 \mathrm{MHz}, \mathrm{CDCl}_{3}\right): \delta=148.5$ (C2), 137.3 (C4), 134.6 (C1), 129.0 (C7), 128.7 (C6), 126.4 (C5), 24.2 (C3) ppm; IR (ATR): $\tilde{v}=3099$ (w), 2987 (w), 2916 (w), 1635 (w), 1600 (w), $1576(\mathrm{w}), 1515$ (s), $1431(\mathrm{~m}), 1395$ (w), $1374(\mathrm{w}), 1337(\mathrm{~s}), 1209(\mathrm{w}), 1182(\mathrm{w}), 1158(\mathrm{w}), 1077$ (w), 1027 (w), 1001 (w), 956 (w), 916 (w), 823 (m), 774 (s), 752 (m), 696 (s) cm ${ }^{-1}$; GC-EI-MS: $\mathrm{m} / \mathrm{z}$ : $[\mathrm{M}-\mathrm{H}]^{+}$calcd. for $\mathrm{C}_{9} \mathrm{H}_{8} \mathrm{NO}_{2}{ }^{+}:$162.05496; found: 162.05493 . The analytical data are in agreement with the literature. ${ }^{[10]}$

\section{(Z)-1-Methyl-4-(1-nitroprop-1-en-2-yl)benzene (Z-2)}

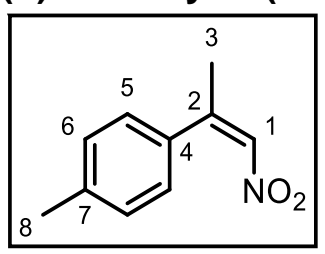

Prepared according to general procedure C, E-2 $(89 \mathrm{mg})$ was converted to $\mathbf{Z - 2}$ yielding a yellow oil as a mixture of isomers $(72.9 \mathrm{mg}, 0.41 \mathrm{mmol}$, $82 \%, Z: E=88: 12)$.

$\mathbf{R}_{\boldsymbol{f}}=0.45\left(\mathrm{Et}_{2} \mathrm{O}: n\right.$-pentane, 5:95); ${ }^{1} \mathrm{H}-\mathbf{N M R}\left(600 \mathrm{MHz}, \mathrm{CDCl}_{3}\right): \delta=7.22-$ $7.19(\mathrm{~m}, 2 \mathrm{H}, \mathrm{H} 6), 7.12-7.10(\mathrm{~m}, 2 \mathrm{H}, \mathrm{H} 5), 7.05\left(\mathrm{q},{ }^{4} \mathrm{~J}_{\mathrm{HH}}=1.4 \mathrm{~Hz}, 1 \mathrm{H}, \mathrm{H} 1\right)$, $2.38(\mathrm{~s}, 3 \mathrm{H}, \mathrm{H} 8), 2.17\left(\mathrm{~d},{ }^{4} \mathrm{JHH}_{\mathrm{HH}} 1.5 \mathrm{~Hz}, 3 \mathrm{H}, \mathrm{H} 3\right) \mathrm{ppm} ;{ }^{13} \mathrm{C}\left\{{ }^{1} \mathrm{H}\right\}-N M R(151$

$\mathrm{MHz}, \mathrm{CDCl}_{3}$ ): $\delta=148.5$ (C2), 139.2 (C7), 134.3 (C1), 134.2 (C4), 129.4 (C5/6), 126.5 (C5/6), 24.2 (C3), 21.5(C8) ppm; IR (ATR): $\tilde{v}=3099$ (w), 3029 (w), $2982(w), 2922(w), 1736(w), 1687$ $(\mathrm{w}), 1632(\mathrm{~m}), 1556(\mathrm{w}), 1518(\mathrm{~s}), 1432(\mathrm{~m}), 1398(\mathrm{w}), 1374(\mathrm{~m}), 1335(\mathrm{~s}), 1216(\mathrm{w}), 1203(\mathrm{w})$, 1187 (w), 1155 (w), 1085 (w), $1033(\mathrm{~m}), 1020$ (w), $955(\mathrm{~m}), 817(\mathrm{~s}), 764(\mathrm{~m}), 711(\mathrm{~m}) \mathrm{cm}^{-1}$; GC-EI-MS: $m / z$ : $[\mathrm{M}-\mathrm{H}]^{+}$calcd. for $\mathrm{C}_{10} \mathrm{H}_{10} \mathrm{NO}_{2}{ }^{+}:$176.07061; found: 176.07058 .

\section{(Z)-1-Methoxy-4-(1-nitroprop-1-en-2-yl)benzene (Z-3)}

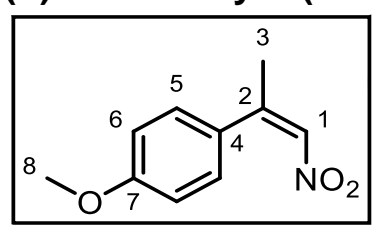

Prepared according to general procedure C, $E-3 \quad(97 \mathrm{mg})$ was converted to $Z \mathbf{Z} 3$ yielding a yellow oil as a mixture of isomers $(86.7 \mathrm{mg}$, $0.45 \mathrm{mmol}, 90 \%, Z: E=88: 12)$.

$\mathbf{R}_{\boldsymbol{f}}=0.16\left(\mathrm{Et}_{2} \mathrm{O}: n\right.$-pentane, 5:95); ${ }^{1} \mathrm{H}-\mathrm{NMR}\left(500 \mathrm{MHz}, \mathrm{CDCl}_{3}\right): \delta=7.20$ $-7.16(\mathrm{~m}, 2 \mathrm{H}, \mathrm{H} 5), 7.03\left(\mathrm{~d},{ }^{4} \mathrm{~J}_{\mathrm{HH}}=1.4 \mathrm{~Hz}, 1 \mathrm{H}, \mathrm{H} 1\right), 6.93-6.90(\mathrm{~m}, 2 \mathrm{H}$, $\mathrm{H} 6), \quad 3.83(\mathrm{~s}, 3 \mathrm{H}, \mathrm{H} 8), 2.17\left(\mathrm{~d},{ }^{4} \mathrm{~J}_{\mathrm{HH}}=1.4 \mathrm{~Hz}, 3 \mathrm{H}, \mathrm{H} 3\right) \mathrm{ppm}$; ${ }^{13} \mathrm{C}\left\{{ }^{1} \mathrm{H}\right\}-N M R\left(126 \mathrm{MHz}, \mathrm{CDCl}_{3}\right): \delta=160.4$ (C7), 147.9 (C2), 134.0 (C1), 129.0 (C4), 128.5 (C5), 114.1 (C6), 55.5 (C8), 24.0 (C3) ppm; IR (ATR): $\tilde{v}=3098$ (w), 2958 (w), 2840 (w), 1606 (m), 1575 (w), 1513 (s), 1463 (m), 1441 (w), 1417 (w), 1334 (s), 1292 (s), 1246 (s), 1178 (s), 1115 (w), 1085 (w), 1028 (s), 955 (w), 831 (s), 808 (s), 764 (m), 728 (w), 709 (w) cm-1; GC-EI-MS: $\mathrm{m} / \mathrm{z}:[\mathrm{M}-\mathrm{H}]^{+}$calcd. for $\mathrm{C}_{10} \mathrm{H}_{11} \mathrm{NO}_{3}{ }^{+}$: 193.07334; found: 193.07329. 
(Z)-1-Fluoro-4-(1-nitroprop-1-en-2-yl)benzene (Z-4)

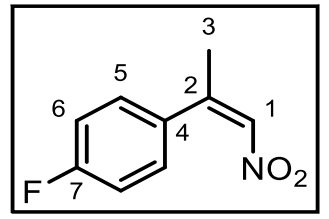

Prepared according to general procedure C, $\boldsymbol{E}-4(91 \mathrm{mg})$ was converted to Z-4 yielding a yellow oil as a mixture of isomers $(68.0 \mathrm{mg}, 0.38 \mathrm{mmol}$, $75 \%, Z: E=88: 12)$.

$\mathbf{R}_{f}=0.22\left(\mathrm{Et}_{2} \mathrm{O}: n\right.$-pentane, 5:95); ${ }^{1} \mathrm{H}-\mathrm{NMR}\left(500 \mathrm{MHz}, \mathrm{CDCl}_{3}\right): \delta=7.22-$ $7.17(\mathrm{~m}, 2 \mathrm{H}, \mathrm{H} 5), 7.12-7.07(\mathrm{~m}, 2 \mathrm{H}, \mathrm{H} 6), 7.06\left(\mathrm{q},{ }^{4} \mathrm{~J}_{\mathrm{HH}}=1.4 \mathrm{~Hz}, 1 \mathrm{H}, \mathrm{H} 1\right)$, $2.18\left(\mathrm{~d},{ }^{4} \mathrm{~J}_{\mathrm{HH}}=1.4 \mathrm{~Hz}, 3 \mathrm{H}, \mathrm{H} 3\right) \mathrm{ppm} ;{ }^{13} \mathrm{C}\left\{{ }^{1} \mathrm{H}\right\}-\mathrm{NMR}\left(126 \mathrm{MHz}, \mathrm{CDCl}_{3}\right)$ : $\delta=163.0\left(\mathrm{~d},{ }^{1} J_{\mathrm{CF}}=249.0 \mathrm{~Hz}, \mathrm{C} 7\right), 147.4(\mathrm{C} 2), 134.8(\mathrm{C} 1), 133.0\left(\mathrm{~d},{ }^{4} J_{\mathrm{CF}}=3.6 \mathrm{~Hz}, \mathrm{C} 4\right), 128.6$ $\left(\mathrm{d},{ }^{3} \mathrm{~J}_{\mathrm{CF}}=8.4 \mathrm{~Hz}, \mathrm{C} 5\right), 115.8\left(\mathrm{~d},{ }^{2} \mathrm{~J}_{\mathrm{CF}}=21.9 \mathrm{~Hz}, \mathrm{C} 6\right), 24.2(\mathrm{C} 3)$ ppm; ${ }^{19} \mathrm{~F}-\mathrm{NMR}\left(470 \mathrm{MHz}, \mathrm{CDCl}_{3}\right)$ : $\delta=-112.06(\mathrm{~m})$ ppm; IR (ATR): $\tilde{v}=3105(\mathrm{w}), 2985(\mathrm{w}), 2866(\mathrm{w}), 1635(\mathrm{w}), 1603(\mathrm{~m}), 1557(\mathrm{w})$, $1516(\mathrm{~s}), 1433(\mathrm{w}), 1405(\mathrm{w}), 1336(\mathrm{~s}), 1302(\mathrm{w}), 1223(\mathrm{~s}), 1160(\mathrm{~m}), 1099(\mathrm{w}), 1032(\mathrm{w}), 1015$ (w), $956(\mathrm{w}), 834(\mathrm{~s}), 765(\mathrm{~m}), 713(\mathrm{~m}) \mathrm{cm}^{-1}$; GC-El-MS: $\mathrm{m} / \mathrm{z}$ : [M-H] ${ }^{+}$calcd. for $\mathrm{C}_{9} \mathrm{H}_{8} \mathrm{NO}_{2} \mathrm{~F}^{+}$: 181.05336; found: 181.05328 .

\section{(Z)-1-Chloro-4-(1-nitroprop-1-en-2-yl)benzene (Z-5)}

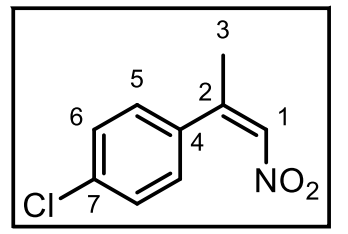

Prepared according to general procedure C, $\mathbf{E}-5(99 \mathrm{mg})$ was converted to $Z-5$ yielding a yellow oil as a mixture of isomers $(80.5 \mathrm{mg}, 0.41 \mathrm{mmol}$, $82 \%, Z: E=92: 08)$.

$\mathbf{R}_{\boldsymbol{f}}=0.22\left(\mathrm{Et}_{2} \mathrm{O}: n\right.$-pentane, $\left.5: 95\right) ;{ }^{1} \mathrm{H}-\mathrm{NMR}\left(600 \mathrm{MHz}, \mathrm{CDCl}_{3}\right): \delta=7.39-$ $7.36(\mathrm{~m}, 2 \mathrm{H}, \mathrm{H} 6), 7.16-7.13(\mathrm{~m}, 2 \mathrm{H}, \mathrm{H} 5), 7.06\left(\mathrm{q},{ }^{4} \mathrm{~J}_{\mathrm{HH}}=1.5 \mathrm{~Hz}, 1 \mathrm{H}, \mathrm{H} 1\right)$, $2.17\left(\mathrm{~d},{ }^{4} \mathrm{JHH}_{\mathrm{HH}}=1.5 \mathrm{~Hz}, 3 \mathrm{H}, \mathrm{H} 3\right) \mathrm{ppm} ;{ }^{3} \mathrm{C}\left\{{ }^{1} \mathrm{H}\right\}-\mathrm{NMR}\left(151 \mathrm{MHz}, \mathrm{CDCl}_{3}\right)$ : $\delta=147.3$ (C2), 135.6 (C4), 135.1 (C7), 134.9 (C1), 129.0 (C6), 128.0 (C5), 24.1 (C3) ppm; IR $($ ATR): $\tilde{v}=3101(w), 2984(w), 1641(w), 1595(w), 1556(w), 1516(s), 1491(\mathrm{~m}), 1432(w), 1399$ (w), $1372(\mathrm{w}), 1335(\mathrm{~s}), 1209(\mathrm{w}), 1093(\mathrm{~m}), 1078(\mathrm{w}), 1031(\mathrm{w}), 1013(\mathrm{~m}), 956(\mathrm{w}), 827(\mathrm{~s}), 772$ (m), $763(\mathrm{~m}), 718(\mathrm{w}) \mathrm{cm}^{-1}$; GC-El-MS: $\mathrm{m} / \mathrm{z}$ : [M-H] ${ }^{+}$calcd. for $\mathrm{C}_{9} \mathrm{H}_{8} \mathrm{NO}_{2} \mathrm{Cl}^{+}$: 197.02381; found: 197.02384. The analytical data are in agreement with the literature. ${ }^{[11]}$

\section{(Z)-1-(1-Nitroprop-1-en-2-yl)-4-(trifluoromethyl)benzene (Z-6)}

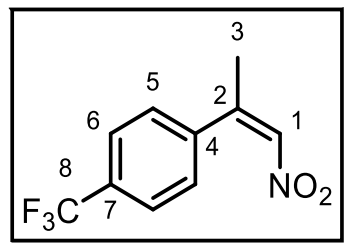

Prepared according to general procedure C, $\boldsymbol{E}-\mathbf{6}(116 \mathrm{mg})$ was converted to $Z \mathbf{Z}-6$ yielding a yellow oil as a mixture of isomers $(101.9 \mathrm{mg}, 0.44 \mathrm{mmol}$, $88 \%, Z: E=89: 11)$.

$\mathbf{R}_{\boldsymbol{f}}=0.14\left(\mathrm{Et}_{2} \mathrm{O}: n\right.$-pentane, 5:95); ${ }^{1} \mathrm{H}-\mathrm{NMR}\left(500 \mathrm{MHz}, \mathrm{CDCl}_{3}\right): \delta=7.67$ $\left(\mathrm{d},{ }^{3} \mathrm{~J}_{\mathrm{HH}}=8.0 \mathrm{~Hz}, 2 \mathrm{H}, \mathrm{H6}\right), 7.31\left(\mathrm{~d},{ }^{3} \mathrm{~J}_{\mathrm{HH}}=8.0 \mathrm{~Hz}, 2 \mathrm{H}, \mathrm{H} 5\right), 7.10\left(\mathrm{q},{ }^{4} \mathrm{~J}_{\mathrm{HH}}=\right.$ $1.4 \mathrm{~Hz}, 1 \mathrm{H}, \mathrm{H} 1), 2.20\left(\mathrm{~d},{ }^{4} \mathrm{JHH}_{\mathrm{HH}}=1.4 \mathrm{~Hz}, 3 \mathrm{H}, \mathrm{H} 3\right) \mathrm{ppm} ;{ }^{13} \mathbf{C}\left\{{ }^{1} \mathrm{H}\right\}-\mathbf{N M R}(126$ $\left.\mathrm{MHz}, \mathrm{CDCl}_{3}\right): \delta=147.2(\mathrm{C} 2), 141.2\left(\mathrm{q},{ }^{5} J_{\mathrm{CF}}=1.2 \mathrm{~Hz}, \mathrm{C} 4\right), 135.3(\mathrm{C} 1), 130.9\left(\mathrm{q},{ }^{2} J_{\mathrm{CF}}=32.7 \mathrm{~Hz}\right.$, C7), 126.8 (C5), 125.8 (q, $\left.{ }^{3} J_{\mathrm{CF}}=3.8 \mathrm{~Hz}, \mathrm{C} 6\right), 122.9$ (q, ${ }^{1} \mathrm{~J}_{\mathrm{CF}}=272.6 \mathrm{~Hz}, \mathrm{C} 8$ ), 24.1 (C3) ppm; ${ }^{19}$ F-NMR (470 MHz, CDCl $): \delta=-62.83$ (s) ppm; IR (ATR): $\tilde{v}=3110(w), 2987(w), 2859(w)$, 1647 (w), $1617(\mathrm{w}), 1525(\mathrm{~s}), 1512(\mathrm{~m}), 1434(\mathrm{w}), 1408(\mathrm{w}), 1352(\mathrm{~m}), 1319(\mathrm{~s}), 1208(\mathrm{w}), 1164$ $(\mathrm{m}), 1110(\mathrm{~s}), 1089(\mathrm{~m}), 1062(\mathrm{~s}), 1032(\mathrm{w}), 1016(\mathrm{~m}), 956(\mathrm{w}), 840(\mathrm{~s}), 767(\mathrm{w}), 730(\mathrm{~m})$; GC-EI-MS: $m / z$ : [M-H] ${ }^{+}$calcd. for $\mathrm{C}_{10} \mathrm{H}_{7} \mathrm{NO}_{2} \mathrm{~F}_{3}{ }^{+}$: 230.04234 ; found: 230.04245 .

\section{(Z)-1-Methyl-2-(1-nitroprop-1-en-2-yl)benzene (Z-7)}

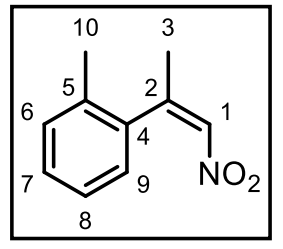

Prepared according to general procedure C, $\boldsymbol{E}-7(89 \mathrm{mg})$ was converted to Z-7 yielding a yellow oil as a mixture of isomers $(53.4 \mathrm{mg}, 0.30 \mathrm{mmol}, 60 \%$, $Z: E=88: 12$ ). The $Z$-isomer could not be isolated cleanly and was not progressed for further hydrogenation analysis.

$\mathbf{R}_{\boldsymbol{f}}=0.29\left(\mathrm{Et}_{2} \mathrm{O}: n\right.$-pentane, 5:95); ${ }^{1} \mathrm{H}-\mathrm{NMR}\left(500 \mathrm{MHz}, \mathrm{CDCl}_{3}\right): \delta=7.27-7.18$ $(\mathrm{m}, 3 \mathrm{H}, \mathrm{H} 6, \mathrm{H} 7, \mathrm{H} 8), 7.16\left(\mathrm{q},{ }^{4} \mathrm{~J}_{\mathrm{HH}}=1.5 \mathrm{~Hz}, 1 \mathrm{H}, \mathrm{H} 1\right), 6.97\left(\mathrm{~d},{ }^{3} \mathrm{~J}_{\mathrm{HH}}=7.6 \mathrm{~Hz}\right.$, $\left.1 \mathrm{H}, \mathrm{H} 9), 2.22(\mathrm{~s}, 3 \mathrm{H}, \mathrm{H} 10), 2.14\left(\mathrm{~d},{ }^{4} \mathrm{~J}_{\mathrm{HH}}=1.5 \mathrm{~Hz}, 3 \mathrm{H}, \mathrm{H} 3\right) \mathrm{ppm} ;{ }^{13} \mathrm{C}^{1}{ }^{1} \mathrm{H}\right\}-\mathrm{NMR}(126 \mathrm{MHz}$, $\left.\mathrm{CDCl}_{3}\right): \delta=149.6$ (C2), 137.5 (C4), 135.6 (C1), 133.6 (C5), 130.4 (C6), 128.3 (C7), 126.2 (C8), 125.3 (C9), 24.1 (C3), 19.2 (C10) ppm; IR (ATR): $\tilde{v}=2925$ (w), 1724 (w), $1642(w), 1601(w)$, $1556(\mathrm{~m}), 1519$ (s), $1488(\mathrm{w}), 1457(\mathrm{w}), 1429(\mathrm{w}), 1372(\mathrm{w}), 1348(\mathrm{~s}), 1335(\mathrm{~m}), 1195(\mathrm{w}), 1125$ (w), 1075 (w), 1045 (w), 958 (w), 830 (w), $773(\mathrm{~m}), 752(\mathrm{~m}), 724(\mathrm{~m}) \mathrm{cm}^{-1}$; GC-El-MS: m/z: $[\mathrm{M}-\mathrm{H}]^{+}$calcd. for $\mathrm{C}_{10} \mathrm{H}_{10} \mathrm{NO}_{2}{ }^{+}:$176.07061; found: 176.07086 . 


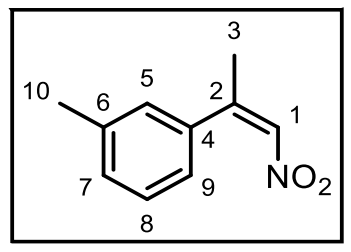

$\left.1.4 \mathrm{~Hz}, 3 \mathrm{H}, \mathrm{H3}) \mathrm{ppm} \cdot{ }^{13} \mathrm{C}^{1} \mathrm{H}\right\}$ NMR $\left(151 \mathrm{MHz}, \mathrm{CDl}_{3}\right): \delta=148.8(\mathrm{C} 2), 138.4(\mathrm{C} 6), 137.3(\mathrm{C} 4)$ 134.4 (C1), 129.8 (C5), 128.6 (C8), 126.9 (C7), 123.5 (C9), 24.3 (C3), 21.6 (C10) ppm; IR $(A T R): \tilde{v}=3099(w), 2922(w), 2864(w), 1635(w), 1603(w), 1583(w), 1556(w), 1517(s), 1431$ (w), $1374(w), 1337(\mathrm{~s}), 1261(\mathrm{w}), 1236(\mathrm{w}), 1101(\mathrm{w}), 1084(\mathrm{w}), 1034(\mathrm{w}), 957(\mathrm{w}), 909(\mathrm{w}), 884$ (w), 788 (s), 763 (w), 700 (m) cm $\mathrm{cm}^{-1}$ GC-El-MS: $\mathrm{m} / \mathrm{z}$ : [M-H] $]^{+}$calcd. for $\mathrm{C}_{10} \mathrm{H}_{10} \mathrm{NO}_{2}{ }^{+}:$176.07061; found: 176.07054 .

\section{(Z)-1-Chloro-3-(1-nitroprop-1-en-2-yl)benzene (Z-9)}

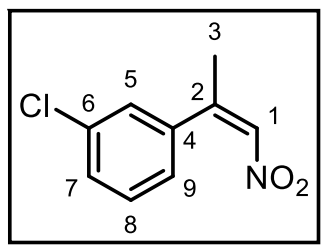

Prepared according to general procedure C, $\boldsymbol{E}-9$ (99 mg) was converted to Z-9 yielding a yellow oil as a mixture of isomers $(80.4 \mathrm{mg}, 0.41 \mathrm{mmol}$, $82 \%, Z: E=93: 07)$.

$\mathbf{R}_{\boldsymbol{f}}=0.21\left(\mathrm{Et}_{2} \mathrm{O}: n\right.$-pentane, 5:95); ${ }^{1} \mathrm{H}-\mathbf{N M R}\left(400 \mathrm{MHz}, \mathrm{CDCl}_{3}\right): \delta=7.38-$ $7.29(\mathrm{~m}, 2 \mathrm{H}, \mathrm{H} 5, \mathrm{H} 7), 7.20-7.17(\mathrm{~m}, 1 \mathrm{H}, \mathrm{H} 8), 7.07-7.05(\mathrm{~m}, 2 \mathrm{H}, \mathrm{H} 9$, $\mathrm{H} 1), 2.18\left(\mathrm{~d},{ }^{4} \mathrm{~J}_{\mathrm{HH}}=1.5 \mathrm{~Hz}, 3 \mathrm{H}, \mathrm{H} 3\right) \mathrm{ppm}$;

${ }^{13} \mathrm{C}\left\{{ }^{1} \mathrm{H}\right\}-N M R\left(151 \mathrm{MHz}, \mathrm{CDCl}_{3}\right): \delta=147.1$ (C2), 139.1 (C4), 135.1 (C1), 130.1 (C6), 129.0 (C5), 126.5 (C7, C8), 124.6 (C9), 24.1 (C3) ppm; IR (ATR): $\tilde{v}=3102$ (w), 2920 (w), 1642 (w), 1594 $(\mathrm{w}), 1556(\mathrm{~m}), 1519(\mathrm{~s}), 1473(\mathrm{w}), 1432(\mathrm{w}), 1415(\mathrm{w}), 1373(\mathrm{w}), 1345(\mathrm{~s}), 1334(\mathrm{~s}), 1301(\mathrm{w})$, $1209(\mathrm{w}), 1168(\mathrm{w}), 1106(\mathrm{w}), 1077(\mathrm{~m}), 1034(\mathrm{w}), 999(\mathrm{w}), 957(\mathrm{w}), 934(\mathrm{w}), 882(\mathrm{~m}), 819(\mathrm{~m})$, 787 (s), $763(\mathrm{~m}), 719$ (w), 691 (s), $678(\mathrm{~m}) \mathrm{cm}^{-1}$; GC-EI-MS: $\mathrm{m} / \mathrm{z}:[\mathrm{M}-\mathrm{H}]^{+}$calcd. for $\mathrm{C}_{9} \mathrm{H}_{7} \mathrm{NO}_{2} \mathrm{Cl}^{+}$: 196.01598; found: 196.01592.

\section{(Z)-(1-Cyclopropyl-2-nitrovinyl)benzene (Z-10)}

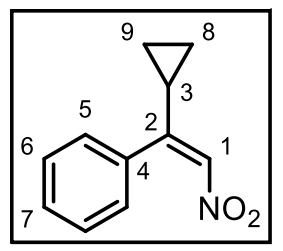

Prepared according to general procedure C, $\boldsymbol{E}-10(95 \mathrm{mg})$ was converted to Z-10 yielding a yellow oil as a mixture of isomers $(78.3 \mathrm{mg}, 0.41 \mathrm{mmol}, 83 \%$, $Z: E=86: 14)$.

$\mathbf{R}_{\boldsymbol{f}}=0.18\left(\mathrm{Et}_{2} \mathrm{O}: n\right.$-pentane, 5:95); ${ }^{1} \mathrm{H}-\mathrm{NMR}\left(500 \mathrm{MHz}, \mathrm{CDCl}_{3}\right): \delta=7.41-7.36$ $(\mathrm{m}, 3 \mathrm{H}, \mathrm{H} 6, \mathrm{H} 7), 7.13(\mathrm{~s}, 1 \mathrm{H}, \mathrm{H} 1), 7.11-7.08(\mathrm{~m}, 2 \mathrm{H}, \mathrm{H} 5), 1.76-1.70(\mathrm{~m}$, $1 \mathrm{H}, \mathrm{H} 3), 0.97-0.92(\mathrm{~m}, 2 \mathrm{H}, \mathrm{H} 8 / 9), 0.69-0.62(\mathrm{~m}, 2 \mathrm{H}, \mathrm{H} 8 / 9) \mathrm{ppm}$; ${ }^{13} \mathrm{C}\left\{{ }^{1} \mathrm{H}\right\}-N M R\left(126 \mathrm{MHz}, \mathrm{CDCl}_{3}\right): \delta=156.1$ (C2), $134.0(\mathrm{C} 4), 133.9(\mathrm{C} 1), 128.6(\mathrm{C} 7), 128.4(\mathrm{C} 6)$, 127.1 (C5), 17.6 (C3), 7.3 (C8, C9) ppm; IR (ATR): v = 3092 (w), 3019 (w), $2972(w), 2926(w)$, $2856(w), 1621(w), 1597(w), 1576(w), 1514(s), 1443(w), 1424(w), 1369(w), 1335(s), 1216$ $(\mathrm{m}), 1176(\mathrm{w}), 1158(\mathrm{w}), 1115(\mathrm{w}), 1075(\mathrm{w}), 1060(\mathrm{w}), 1035(\mathrm{w}), 995(\mathrm{~m}), 915(\mathrm{w}), 871(\mathrm{~m}), 815$ $(\mathrm{m}), 772(\mathrm{~m}), 744(\mathrm{~m}), 721(\mathrm{~m}), 699$ (s), 669 (w) $\mathrm{cm}^{-1}$; GC-EI-MS: m/z: [M-O] ${ }^{+}$calcd. for $\mathrm{C}_{11} \mathrm{H}_{11} \mathrm{NO}^{+}:$173.08352; found: 178.08346 .

\section{(Z)-(2-Nitrovinyl)benzene (Z-11)}

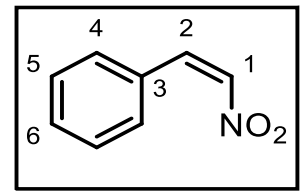

Prepared according to general procedure $\mathbf{C},(E)$-(2-nitrovinyl)benzene (75 mg) was converted to Z-11 yielding a yellow oil as an inseparable mixture of isomers $(49.9 \mathrm{mg}, 0.33 \mathrm{mmol}, 67 \%, Z: E=46: 54)$.

$\mathbf{R}_{\boldsymbol{f}}=0.68\left(\mathrm{Et}_{2} \mathrm{O}: n\right.$-pentane, 5:95);

${ }^{1} \mathrm{H}-\mathrm{NMR}\left(500 \mathrm{MHz}, \mathrm{CDCl}_{3}\right): \delta=7.53-7.43(\mathrm{~m}, 5 \mathrm{H}, \mathrm{H} 4, \mathrm{H} 5, \mathrm{H} 6), 6.97$ (d, $\left.{ }^{3} J_{\mathrm{HH}}=9.5 \mathrm{~Hz}, 1 \mathrm{H}, \mathrm{H} 1\right), 6.78\left(\mathrm{~d},{ }^{3} \mathrm{~J}_{\mathrm{HH}}=9.5 \mathrm{~Hz}, 1 \mathrm{H}, \mathrm{H} 2\right) \mathrm{ppm} ;{ }^{13} \mathbf{C}\left\{{ }^{1} \mathrm{H}\right\}-\mathrm{NMR}\left(126 \mathrm{MHz}, \mathrm{CDCl}_{3}\right)$ : $\delta=135.9$ (C1), 133.9 (C2), 130.8 (C3), 130.6 (C5), 130.0 (C4), 128.7 (C6) ppm; IR (ATR): $\tilde{v}=3110(w), 3035(w), 1634(w), 1602(w), 1577(w), 1549(w), 1516(s), 1495(m), 1449(w)$, $1374(w), 1339(\mathrm{~s}), 1260(\mathrm{w}), 1201(\mathrm{w}), 1180(\mathrm{w}), 1072(\mathrm{w}), 1029(\mathrm{w}), 964(\mathrm{~m}), 837(\mathrm{w}), 792(\mathrm{w})$, $764(\mathrm{~m}), 737(\mathrm{w}), 696(\mathrm{~s}), 680(\mathrm{~m}) \mathrm{cm}^{-1}$; GC-El-MS: $\mathrm{m} / \mathrm{z}$ : [M] ${ }^{+}$calcd. for $\mathrm{C}_{8} \mathrm{H}_{7} \mathrm{NO}_{2}{ }^{+}$: 149.05; found: 149.08. The analytical data are in agreement with the literature. ${ }^{[12]}$ 


\section{(Z)-1-Fluoro-2-(2-nitrovinyl)benzene (E-12)}

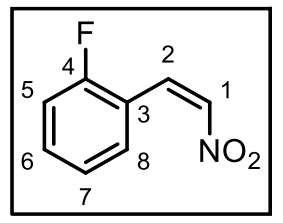

Prepared according to general procedure C, E-12 $(84 \mathrm{mg})$ was converted to Z-12 yielding a yellow oil as an inseparable mixture of isomers $(23.1 \mathrm{mg}$, $0.14 \mathrm{mmol}, 28 \%, Z: E=67: 33)$.

$\mathbf{R}_{\boldsymbol{f}}=0.65\left(\mathrm{Et}_{2} \mathrm{O}: n\right.$-pentane, $\left.5: 95\right) ;{ }^{1} \mathrm{H}-\mathrm{NMR}\left(600 \mathrm{MHz}, \mathrm{CDCl}_{3}\right): \delta=7.50-7.46$ (m, 1H, H8), $7.44-7.39(\mathrm{~m}, 1 \mathrm{H}, \mathrm{C} 6), 7.20-7.15(\mathrm{~m}, 1 \mathrm{H}, \mathrm{C} 7), 7.15-7.09$ $(\mathrm{m}, 1 \mathrm{H}, \mathrm{C}), 7.07\left(\mathrm{~d},{ }^{3} \mathrm{~J}_{\mathrm{HH}}=9.2 \mathrm{~Hz}, 1 \mathrm{H}, \mathrm{C} 2\right), 6.89\left(\mathrm{~d},{ }^{3} \mathrm{JHH}_{\mathrm{HH}}=9.2 \mathrm{~Hz}, 1 \mathrm{H}\right.$, C1) ppm; ${ }^{13} \mathrm{C}\left\{{ }^{1} \mathrm{H}\right\}-\mathrm{NMR}\left(151 \mathrm{MHz}, \mathrm{CDCl}_{3}\right): \delta=160.5\left(\mathrm{~d},{ }^{1} \mathrm{JFF}_{\mathrm{CF}}=251.8 \mathrm{~Hz}, \mathrm{C} 4\right), 138.0\left(\mathrm{~d},{ }^{3} \mathrm{~J}_{\mathrm{CF}}=\right.$ $1.1 \mathrm{~Hz}, \mathrm{C} 2), 132.3\left(\mathrm{~d},{ }^{3} \mathrm{~J}_{\mathrm{CF}}=8.8 \mathrm{~Hz}, \mathrm{C} 6\right), 130.6\left(\mathrm{~d},{ }^{3} \mathrm{~J}_{\mathrm{CF}}=1.9 \mathrm{~Hz}, \mathrm{C} 8\right), 127.0\left(\mathrm{~d},{ }^{4} \mathrm{~J}_{\mathrm{CF}}=3.6 \mathrm{~Hz}\right.$, C1), $124.3\left(\mathrm{~d},{ }^{4} \mathrm{~J}_{\mathrm{CF}}=3.5 \mathrm{~Hz}, \mathrm{C} 7\right), 119.3\left(\mathrm{~d},{ }^{2} \mathrm{~J}_{\mathrm{CF}}=13.5 \mathrm{~Hz}\right), 115.9\left(\mathrm{~d},{ }^{2} \mathrm{~J}_{\mathrm{CF}}=21.6 \mathrm{~Hz}, \mathrm{C} 5\right) \mathrm{ppm}$; ${ }^{19} \mathrm{~F}-\left\{{ }^{1} \mathrm{H}\right\}-\mathrm{NMR}\left(470 \mathrm{MHz}, \mathrm{CDCl}_{3}\right): \delta=-111.60$ (s) ppm; IR (ATR): $\tilde{v}=3110(\mathrm{w}), 2925$ (w), 2855 (w), $1637(\mathrm{w}), 1611(\mathrm{w}), 1579(\mathrm{w}), 1517(\mathrm{~s}), 1486(\mathrm{~m}), 1456(\mathrm{~m}), 1372(\mathrm{w}), 1339(\mathrm{~s}), 1283(\mathrm{w})$, $1222(w), 1155(w), 1098(w), 1033(w), 964(m), 890(w), 865(w), 833(w), 820(w), 809(w)$, 755 (s), $732(\mathrm{~m}) \mathrm{cm}^{-1}$; GC-El-MS: $\mathrm{m} / \mathrm{z}:[\mathrm{M}]^{+}$calcd. for $\mathrm{C}_{8} \mathrm{H}_{8} \mathrm{FNO}_{2}{ }^{+}:$167.04; found: 167.07 . 


\section{Synthesis of nitroalkanes}

\section{General procedure $D$ for the preparation of the racemic nitroalkanes}

Following a modified literature procedure, ${ }^{[1]}$ the $E$-nitroalkane $E-1-10$ (1 eq.) was dissolved in THF:MeOH (10:1, $0.2 \mathrm{M}$ ) and $\mathrm{NaBH}_{4}$ (1.5 eq.) was added portionwise (gas evolution). The reaction mixture was stirred at room temperature and monitored by TLC. Upon conversion of the starting material and cease of the gas evolution, the reaction was quenched with $\mathrm{H}_{2} \mathrm{O}$. The aqueous layer was extracted with $\mathrm{CH}_{2} \mathrm{Cl}_{2}(3 \mathrm{x})$ and the combined organic layers were washed with brine, dried over $\mathrm{MgSO}_{4}$, filtered and concentrated in vacuo. No further purification was required.

\section{General procedure $E$ for the preparation of the (S)-nitroalkanes}

Following a modified literature procedure, ${ }^{[1]}$ the $E$-nitroalkene $(0.2 \mathrm{mmol}, 1$ eq. $)$ was weighed into a $2 \mathrm{~mL}$ vial. The specified catalyst (A: $3.8 \mathrm{mg}$ or B: $4.2 \mathrm{mg}, 0.01 \mathrm{mmol}, 0.05$ eq.) and the Hantzsch ester (di-tert-butyl 2,6-dimethyl-1,4-dihydropyridine-3,5-dicarboxylate, HE) (68 mg, $0.22 \mathrm{mmol}, 1.1$ eq.) were added and the walls of the vial were rinsed with toluene $(616 \mu \mathrm{L}$, $0.32 \mathrm{M})$. A rubber septum and parafilm were used to seal the vial, which was then placed in a preheated metal heating block at $40^{\circ} \mathrm{C}$ for $48 \mathrm{~h}$ (Figure 3). After that time, the crude reaction mixture was filtered through a plug of silica, which was rinsed with $\mathrm{Et}_{2} \mathrm{O}: n$-pentane $(2: 98)$ to remove the catalyst and most of the oxidized Hantzsch ester. The filtrate was concentrated in vacuo and purified by column chromatography to yield the $(S)$-nitroalkane. Reported yields and stereoselectivities are an average of two duplicate reactions. The enantiomeric ratio was determined via chiral HPLC and a spectrum of a single reaction is shown.<smiles>Cc1ccc(C)n1[C@H]1CCCC[C@H]1NC(=S)N[C@H](C(=O)N(C)C)C(C)C</smiles><smiles>CC(C)[C@H](NC(=S)Nc1cc(C(F)(F)F)cc(C(F)(F)F)c1)C(=O)N(C)C</smiles><smiles>CC1=C(C(=O)OC(C)(C)C)CC(C(=O)OC(C)(C)C)=C(C)N1</smiles>

Hantzsch ester

Figure 4: Structures of the used catalysts and Hantzsch ester.

\section{General procedure $F$ for the preparation of the $(R)$-nitroalkanes}

Following a modified literature procedure, ${ }^{[1]}$ the $Z$-nitroalkene $(0.2 \mathrm{mmol}, 1$ eq. $)$ was weighed into a $2 \mathrm{~mL}$ vial. The specified catalyst (A: $3.8 \mathrm{mg}$ or B: $4.2 \mathrm{mg}, 0.01 \mathrm{mmol}, 0.05$ eq.) and the Hantzsch ester (di-tert-butyl 2,6-dimethyl-1,4-dihydropyridine-3,5-dicarboxylate, HE) (68 mg, $0.22 \mathrm{mmol}, 1.1$ eq.) were added and the walls of the vial were rinsed with toluene $(616 \mu \mathrm{L}$, $0.32 \mathrm{M}$ ). A rubber septum and parafilm was used to seal the vial which was then placed in a preheated metal heating block at $40{ }^{\circ} \mathrm{C}$ for $48 \mathrm{~h}$ (Figure 3). After that time, the crude reaction mixture was filtered through a plug of silica, which was rinsed with $\mathrm{Et}_{2} \mathrm{O}: n$-pentane $(2: 98)$ to remove the catalyst and most of the oxidized Hantzsch ester. The filtrate was concentrated in vacuo and purified by column chromatography to yield the $(R)$-nitroalkane. Reported yields and stereoselectivities are an average of two duplicate reactions. The enantiomeric ratio was determined via chiral HPLC and a spectrum of a single reaction is shown.<smiles>Cc1ccc(C)n1[C@@H]1CCCC[C@H]1NC(=S)N[C@H](C(=O)N(C)C)C(C)C</smiles><smiles>CC(C)[C@H](NC(=S)Nc1cc(C(F)(F)F)cc(C(F)(F)F)c1)C(=O)N(C)C</smiles>

B<smiles>CC1=C(C(=O)OC(C)(C)C)CC(C(=O)OC(C)(C)C)=C(C)N1</smiles>

Hantzsch ester

Figure 5: Structures of the used catalysts and Hantzsch ester. 


\section{Preparation and analytical data of the nitroalkanes}

\section{rac-(1-Nitropropan-2-yl)benzene (rac-13)}

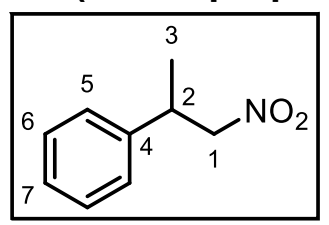

Following general procedure $\mathbf{D}, \boldsymbol{E}-1$ ( $33 \mathrm{mg}, 0.2 \mathrm{mmol}, 1 \mathrm{eq}$.) was treated with $\mathrm{NaBH}_{4}(11 \mathrm{mg}, 1.5 \mathrm{mmol}, 1.5$ eq.) in THF:MeOH $(10: 1,1 \mathrm{~mL})$ to obtain rac-13 after $4 \mathrm{~h}$ as a colorless oil (19.0 mg, $0.12 \mathrm{mmol}, 58 \%$ ).

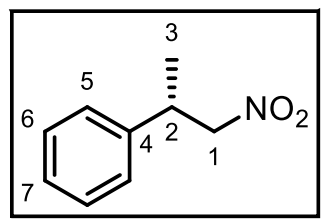

(S)-(1-Nitropropan-2-yl)benzene ((S)-13)

Prepared according to general procedure E, E-1 (33 mg) was converted to (S)-13, a colorless oil, using the catalyst stated in Table 1. The outcome for the given catalyst is summarized in Table 1 with the enantiomeric ratio determined by chiral HPLC (Reprosil OM, $n$-hexane:PrOH 97:3, $1.0 \mathrm{~mL} / \mathrm{min}, \lambda=210 \mathrm{~nm}$ ).

Table 1: Reaction outcome for the hydrogenation of $E-1$ to $(S)-13$.

\begin{tabular}{|c|c|c|c|c|c|c|c|}
\hline entry & catalyst & yield & & & er & $t_{R}$ (minor) & $t_{R}$ (major) \\
\hline 1 & $A$ & $31.6 \mathrm{mg}$ & $0.19 \mathrm{mmol}$ & $96 \%$ & $04: 96$ & $10.561 \mathrm{~min}$ & $13.396 \mathrm{~min}$ \\
\hline 2 & B & $28.5 \mathrm{mg}$ & $0.17 \mathrm{mmol}$ & $86 \%$ & 09:91 & $10.511 \mathrm{~min}$ & $13.350 \mathrm{~min}$ \\
\hline
\end{tabular}

(R)-(1-Nitropropan-2-yl)benzene $((R)$-13)

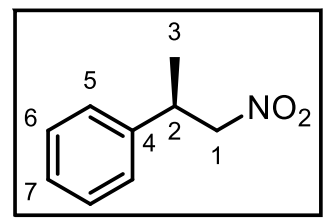

Prepared according to general procedure $\mathbf{F}, \mathbf{Z}-\mathbf{1}(33 \mathrm{mg}$ ) was converted to $(\boldsymbol{R})-13$, a colorless oil, using the catalyst stated in Table 2 . The outcome for the given catalyst is summarized in Table 2 with the enantiomeric ratio determined by chiral HPLC (Reprosil OM, $n$-hexane:IPrOH 97:3, $1.0 \mathrm{~mL} / \mathrm{min}, \lambda=210 \mathrm{~nm}$ ).

Table 2: Reaction outcome for the hydrogenation of $Z-1$ to $(\boldsymbol{R})-\mathbf{1 3}$.

\begin{tabular}{|c|c|c|c|c|c|c|c|}
\hline entry & catalyst & yield & & & er & $t_{R}($ major $)$ & $t_{R}($ minor $)$ \\
\hline 1 & A & $29.0 \mathrm{mg}$ & $0.18 \mathrm{mmol}$ & $88 \%$ & $84: 16$ & $10.544 \mathrm{~min}$ & $13.454 \mathrm{~min}$ \\
\hline 2 & B & $31.0 \mathrm{mg}$ & $0.19 \mathrm{mmol}$ & $94 \%$ & 93:07 & $10.491 \mathrm{~min}$ & $13.388 \mathrm{~min}$ \\
\hline
\end{tabular}

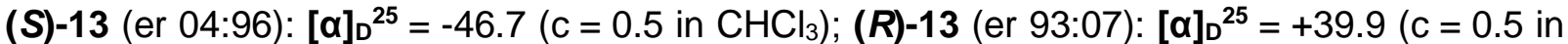
$\left.\mathrm{CHCl}_{3}\right) ; \mathbf{R}_{f}=0.53\left(\mathrm{Et}_{2} \mathrm{O}: n\right.$-pentane, 5:95); ${ }^{1} \mathrm{H}-\mathrm{NMR}\left(400 \mathrm{MHz}, \mathrm{CDCl}_{3}\right): \delta=7.39-7.30(\mathrm{~m}, 2 \mathrm{H}$, $\mathrm{H} 6), 7.30-7.21(\mathrm{~m}, 3 \mathrm{H}, \mathrm{H} 5, \mathrm{H} 7), 4.59-4.46(\mathrm{~m}, 2 \mathrm{H}, \mathrm{H} 1), 3.64\left(\mathrm{~h},{ }^{3} J_{\mathrm{HH}}=7.0 \mathrm{~Hz}, 1 \mathrm{H}, \mathrm{H} 2\right), 1.39$ (d, $\left.{ }^{3} J_{\mathrm{HH}}=7.0 \mathrm{~Hz}, 3 \mathrm{H}, \mathrm{H} 3\right) \mathrm{ppm} ;{ }^{13} \mathrm{C}\left\{{ }^{1} \mathrm{H}\right\}-N M R\left(101 \mathrm{MHz}, \mathrm{CDCl}_{3}\right): \delta=141.0(\mathrm{C} 4), 129.1$ (C6), 127.7 (C7), 127.1 (C5), 82.0 (C1), 38.8 (C2), 18.9 (C3) ppm; IR (ATR): $\tilde{v}=3673$ (w), 2972 (w), 1604 (w), $1546(\mathrm{~s}), 1495(\mathrm{w}), 1454(\mathrm{~m}), 1431(\mathrm{w}), 1377(\mathrm{~m}), 1332(\mathrm{w}), 1310(\mathrm{w}), 1291(\mathrm{w}), 1226$

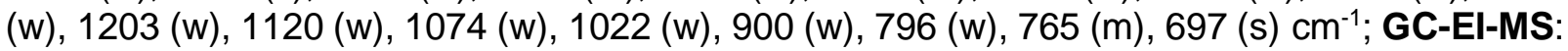
$\mathrm{m} / \mathrm{z}$ : [M] ${ }^{+}$calcd. for $\mathrm{C}_{9} \mathrm{H}_{11} \mathrm{NO}_{2}{ }^{+}: 165.10$; found: 165.16 . The analytical data are in agreement with the literature. ${ }^{[11]}$ 


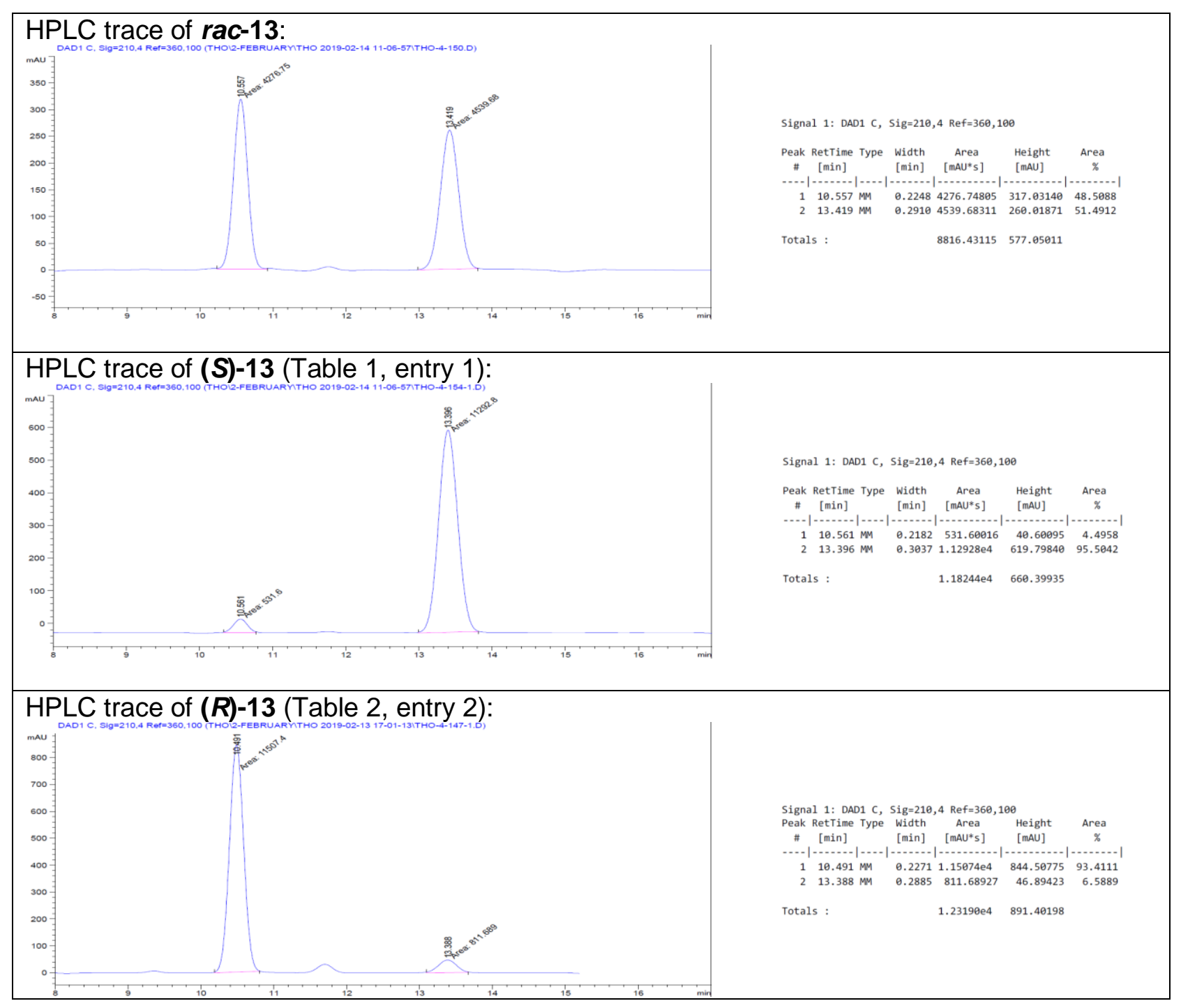

Figure 6: HPLC traces of 13; top: rac-13, middle: (S)-13 (Table 1, entry 1), bottom: $(\boldsymbol{R})$-13 (Table 2, entry 2). 


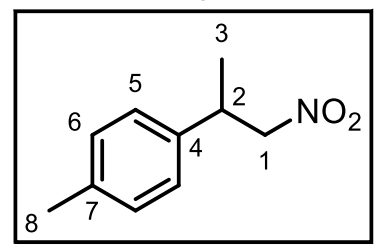
Following general procedure $\mathbf{D}, \mathbf{E}-2$ (35 mg, $0.2 \mathrm{mmol}$, 1 eq.) was treated with $\mathrm{NaBH}_{4}(11 \mathrm{mg}, 0.3 \mathrm{mmol}, 1.5$ eq.) in THF:MeOH (10:1, $1.4 \mathrm{~mL})$ to obtain rac-14 after $4 \mathrm{~h}$ as a colorless oil $(11.1 \mathrm{mg}$, $0.06 \mathrm{mmol}, 31 \%)$.

\section{(S)-1-Methyl-4-(1-nitropropan-2-yl)benzene ((S)-14)}

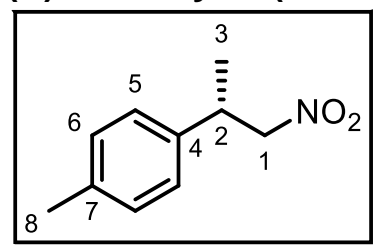

Prepared according to general procedure E, E-2 (35 mg) was converted to (S)-14, a colorless oil, using the catalyst stated in Table 3. The outcome for the given catalyst is summarized in Table 3 with the enantiomeric ratio determined by chiral HPLC (Reprosil OM, $n$-hexane:PrOH 99:1, $1.0 \mathrm{~mL} / \mathrm{min}, \lambda=210 \mathrm{~nm}$ ).

Table 3: Reaction outcome for the hydrogenation of E-2 to (S)-14.

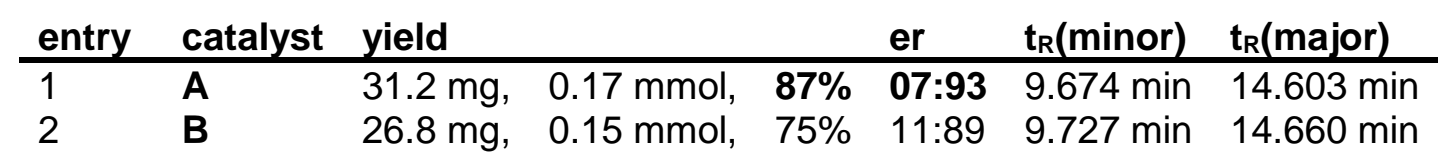

(R)-1-Methyl-4-(1-nitropropan-2-yl)benzene ((R)-14)<smiles>O=[N+]([O-])[C@H](I)c1ccc(CC2CC2)cc1</smiles>
Prepared according to general procedure F, Z-2 $(35 \mathrm{mg})$ was converted to $(\boldsymbol{R})-\mathbf{1 4}$, a colorless oil, using the catalyst stated in Table 4 . The outcome for the given catalyst is summarized in Table 4 with the enantiomeric ratio determined by chiral HPLC (Reprosil OM, $n$-hexane:PrOH 99:1, $1.0 \mathrm{~mL} / \mathrm{min}, \lambda=210 \mathrm{~nm}$ ).

Table 4: Reaction outcome for the hydrogenation of $\mathbf{Z - 2}$ to $(\boldsymbol{R})-\mathbf{1 4}$.

\begin{tabular}{|c|c|c|c|c|c|c|c|}
\hline entry & catalyst & yield & & & er & $t_{R}$ (major) & $t_{R}$ (minor) \\
\hline $1^{\mathrm{a}}$ & A & $26.3 \mathrm{mg}$ & $0.15 \mathrm{mmol}$, & $74 \%$ & $87: 13$ & $11.975 \mathrm{~min}$ & $20.508 \mathrm{~min}$ \\
\hline 2 & B & $33.8 \mathrm{mg}$, & $0.19 \mathrm{mmol}$, & $94 \%$ & $87: 13$ & $9.648 \mathrm{~min}$ & $14.607 \mathrm{~min}$ \\
\hline
\end{tabular}

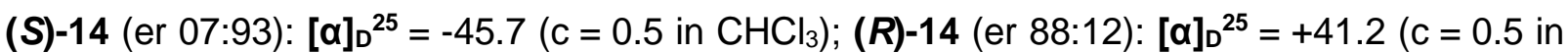
$\left.\mathrm{CHCl}_{3}\right) ; \mathbf{R}_{f}=0.55\left(\mathrm{Et}_{2} \mathrm{O}: n\right.$-pentane, 5:95); ${ }^{1} \mathrm{H}-\mathrm{NMR}\left(400 \mathrm{MHz}, \mathrm{CDCl}_{3}\right): \delta=7.17-7.09(\mathrm{~m}, 4 \mathrm{H}$, $\mathrm{H} 5, \mathrm{H} 6), 4.56-4.43(\mathrm{~m}, 2 \mathrm{H}, \mathrm{H} 1), 3.60\left(\mathrm{se},{ }^{3} \mathrm{~J}_{\mathrm{HH}}=7.0 \mathrm{~Hz}, 1 \mathrm{H}, \mathrm{H} 2\right), 2.33(\mathrm{~s}, 3 \mathrm{H}, \mathrm{H} 8), 1.36(\mathrm{~d}$, $\left.{ }^{3} J_{\mathrm{H}}=7.0 \mathrm{~Hz}, 3 \mathrm{H}\right) \mathrm{ppm} ;{ }^{13} \mathrm{C}\left\{{ }^{1} \mathrm{H}\right\}-\mathrm{NMR}\left(101 \mathrm{MHz}, \mathrm{CDCl}_{3}\right): \delta=138.0(\mathrm{C} 4), 137.4(\mathrm{C} 7), 129.8(\mathrm{C} 6)$, 126.9 (C5), 82.2 (C1), 38.5 (C2), 21.2 (C8), 18.9 (C3) ppm; IR (ATR): v = 2972 (w), 2922 (w), 1546 (s), $1515(\mathrm{~m}), 1455(\mathrm{w}), 1431(\mathrm{w}), 1377(\mathrm{~m}), 1310(\mathrm{w}), 1199(\mathrm{w}), 1122(\mathrm{w}), 1032(\mathrm{w}), 1019$ (w), 898 (w), 815 (s), $772(w), 719$ (w), 686 (w) cm ${ }^{-1}$; GC-El-MS: m/z: ([M] calcd. for $\mathrm{C}_{10} \mathrm{H}_{13} \mathrm{NO}_{2}{ }^{+}$: 179.09; found: 179.12 . The analytical data are in agreement with the literature. ${ }^{[13]}$ 


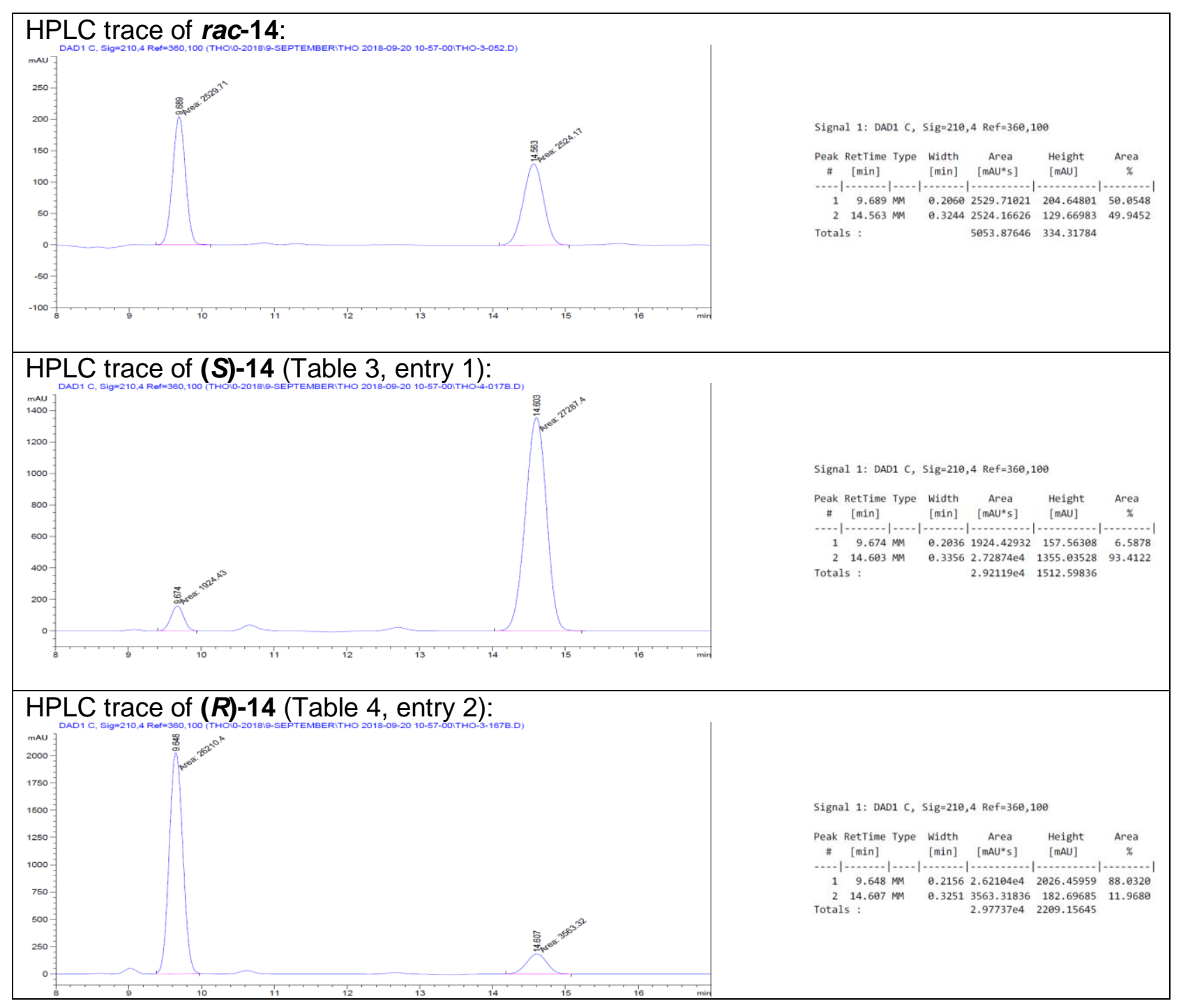

Figure 6: HPLC traces of 14; top: rac-14, middle: (S)-14 (Table 3, entry 1), bottom: $(\boldsymbol{R})$-14 (Table 4, entry 2). 

treated with $\mathrm{NaBH}_{4}$ (9 mg, $0.23 \mathrm{mmol}, 1.5$ eq.) in THF:MeOH (10:1, $1 \mathrm{~mL})$. After stirring for $4 \mathrm{~h}$, a second portion of $\mathrm{NaBH}_{4}(9 \mathrm{mg}$, $0.23 \mathrm{mmol}, 1.5 \mathrm{eq}$.) was added and the reaction mixture was stirred for $1 \mathrm{~h}$ to yield rac-15 as a colorless oil (18.6 mg, $0.10 \mathrm{mmol}, 64 \%)$.

(S)-1-Methoxy-4-(1-nitropropan-2-yl)benzene ((S)-15)

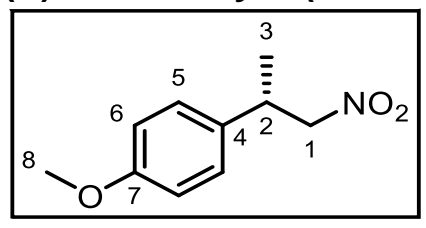

Prepared according to general procedure E, E-3 $(40 \mathrm{mg})$ was converted to (S)-15, a colorless oil, using the catalyst stated in Table 5. The outcome for the given catalyst is summarized in Table 5 with the enantiomeric ratio determined by chiral HPLC (Chiralce ${ }^{\circledR} \mathrm{OJ}-\mathrm{H}, n$-hexane:PrOH 98:2, $1.0 \mathrm{~mL} / \mathrm{min}, \lambda=210 \mathrm{~nm}$ ).

Table 5: Reaction outcome for the hydrogenation of E-3 to (S)-15.

\begin{tabular}{|c|c|c|c|c|c|c|c|}
\hline entry & catalyst & yield & & & er & $t_{R}($ minor $)$ & $t_{R}($ major $)$ \\
\hline 1 & A & $30.3 \mathrm{mg}$, & $0.16 \mathrm{mmol}$, & $78 \%$ & $06: 94$ & $30.029 \mathrm{~min}$ & $35.123 \mathrm{~min}$ \\
\hline
\end{tabular}

(R)-1-Methoxy-4-(1-nitropropan-2-yl)benzene ((R)-15)

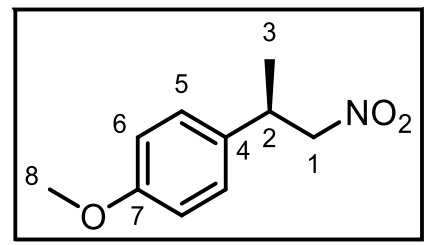

Prepared according to general procedure F, Z-3 (40 mg) was converted to $(\boldsymbol{R})-\mathbf{1 5}$, a colorless oil, using the catalyst stated in Table 6 . The outcome for the given catalyst is summarized in Table 6 with the enantiomeric ratio determined by chiral HPLC (Chiralcel ${ }^{\circledR} \mathrm{OJ}-\mathrm{H}, n$-hexane:iPrOH 98:2, $1.0 \mathrm{~mL} / \mathrm{min}, \lambda=210 \mathrm{~nm}$ ).

Table 6: Reaction outcome for the hydrogenation of $\mathbf{Z - 3}$ to $(\boldsymbol{R}) \mathbf{- 1 5}$.

\begin{tabular}{|c|c|c|c|c|c|c|c|}
\hline entry & catalyst & yield & & & er & $t_{R}($ major $)$ & $t_{R}($ minor $)$ \\
\hline 1 & $A$ & $35.0 \mathrm{mg}$, & $0.18 \mathrm{mmol}$, & $90 \%$ & $85: 15$ & $29.381 \mathrm{~min}$ & $34.895 \mathrm{~min}$ \\
\hline 2 & B & $31.7 \mathrm{mg}$, & $0.16 \mathrm{mmol}$, & $81 \%$ & $87: 13$ & 29:434 min & $34: 923 \mathrm{~min}$ \\
\hline
\end{tabular}

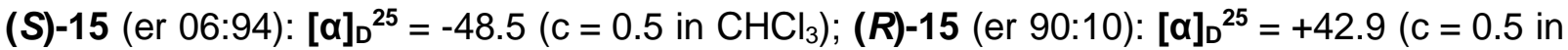
$\left.\mathrm{CHCl}_{3}\right) ; \mathbf{R}_{\boldsymbol{f}}=0.21\left(\mathrm{Et}_{2} \mathrm{O}: n\right.$-pentane, 5:95); ${ }^{1} \mathrm{H}-\mathrm{NMR}\left(400 \mathrm{MHz}, \mathrm{CDCl}_{3}\right): \delta=7.17-7.12(\mathrm{~m}, 2 \mathrm{H}$, $\mathrm{H} 5), 6.89-6.85(\mathrm{~m}, 2 \mathrm{H}, \mathrm{H} 6), 4.54-4.42(\mathrm{~m}, 2 \mathrm{H}, \mathrm{H} 1), 3.79(\mathrm{~s}, 3 \mathrm{H}, \mathrm{H} 8), 3.59\left(\mathrm{~h},{ }^{3} \mathrm{~J}_{\mathrm{HH}}=7.1 \mathrm{~Hz}\right.$, $1 \mathrm{H}, \mathrm{H} 2), 1.36\left(\mathrm{~d},{ }^{3} \mathrm{~J}_{\mathrm{HH}}=7.1 \mathrm{~Hz}, 3 \mathrm{H}, \mathrm{H} 3\right) \mathrm{ppm} ;{ }^{13} \mathrm{C}\left\{{ }^{1} \mathrm{H}\right\}-\mathrm{NMR}\left(101 \mathrm{MHz}, \mathrm{CDCl}_{3}\right): \delta=159.1(\mathrm{C} 7)$, 133.0 (C4), 128.1 (C5), 114.5 (C6), 82.3 (C1), 55.4 (C8), 38.1 (C2), 19.0 (C3) ppm; IR (ATR): $\tilde{v}=2969(w), 2936(w), 2838(w), 1612(w), 1585(w), 1550(s), 1515(s), 1459(w), 1384(w)$, $1300(\mathrm{w}), 1250(\mathrm{~m}), 1181(\mathrm{~m}), 1124(\mathrm{w}), 1038(\mathrm{w}), 1026(\mathrm{w}), 899(\mathrm{w}), 832(\mathrm{~m}), 773(\mathrm{w}), 726(\mathrm{w})$, 696 (w) $\mathrm{cm}^{-1}$; GC-El-MS: $\mathrm{m} / \mathrm{z}$ : [M] ${ }^{+}$calcd. for $\mathrm{C}_{10} \mathrm{H}_{13} \mathrm{NO}_{3}{ }^{+}:$195.08899; found: 195.08889 . The analytical data are in agreement with the literature. ${ }^{[13]}$ 


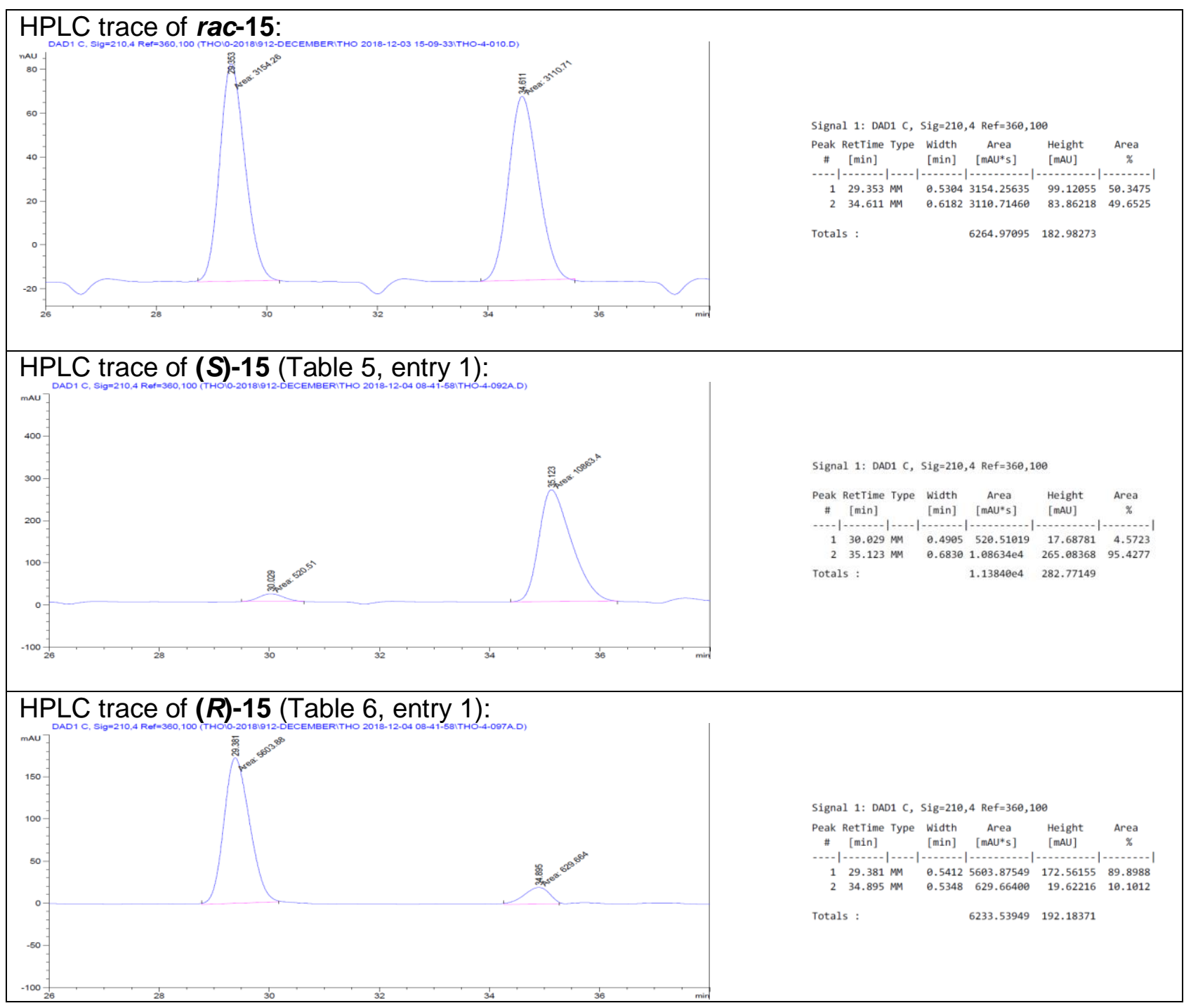

Figure 8: HPLC traces of 15; top: rac-15, middle: (S)-15 (Table 5, entry 1); bottom: $(\boldsymbol{R})$-15 (Table 6, entry 1$)$. 

$2.4 \mathrm{~mL}$ ) to obtain rac-16 after $5 \mathrm{~h}$ as a colorless oil $(14.4 \mathrm{mg}, 0.08 \mathrm{mmol}$, $22 \%)$.

(S)-1-Fluoro-4-(1-nitropropan-2-yl)benzene ((S)-16)

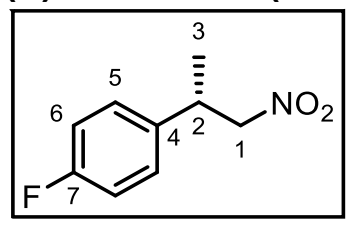

Prepared according to general procedure E, E-4 $(36 \mathrm{mg})$ was converted to (S)-16, a colorless oil, using the catalyst stated in Table 7. The outcome for the given catalyst is summarized in Table 7 with the enantiomeric ratio determined by chiral HPLC (Reprosil Chiral-OM, $n$-hexane:iPrOH 99.7:0.3, $1.0 \mathrm{~mL} / \mathrm{min}, \lambda=210 \mathrm{~nm}$ ).

Table 7: Reaction outcome for the hydrogenation of E-4 to (S)-16.

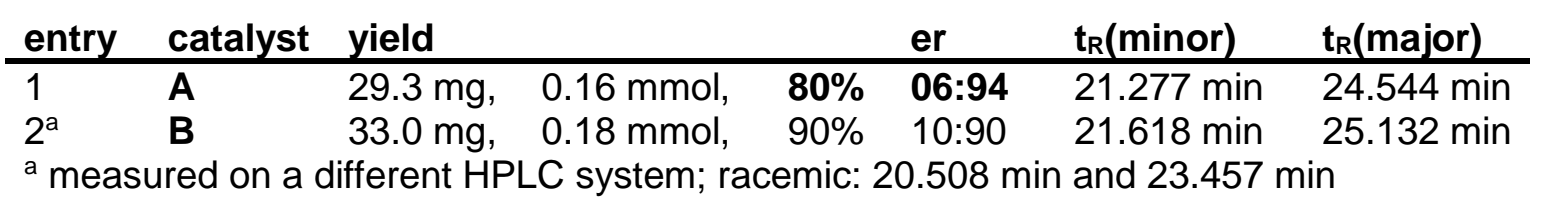

(R)-1-Fluoro-4-(1-nitropropan-2-yl)benzene ((R)-16)<smiles>C[C@H](c1ccc(F)cc1)[N+](=O)[O-]</smiles>
Prepared according to general procedure $\mathbf{F}, \boldsymbol{Z}-\mathbf{4}(36 \mathrm{mg})$ was converted to $(R)-16$, a colorless oil, using the catalyst stated in Table 8 . The outcome for the given catalyst is summarized in Table 8 with the enantiomeric ratio determined by chiral HPLC (Reprosil Chiral-OM, $n$-hexane: $\mathrm{PrOH}$ 99.7:0.3, $1.0 \mathrm{~mL} / \mathrm{min}, \lambda=210 \mathrm{~nm}$ ).

Table 8: Reaction outcome for the hydrogenation of $\mathbf{Z - 4}$ to $(\boldsymbol{R}) \mathbf{- 1 6}$.

\begin{tabular}{|c|c|c|c|c|c|c|c|}
\hline entry & catalyst & yield & & & er & $t_{R}($ major $)$ & $t_{R}($ minor $)$ \\
\hline 1 & A & $35.5 \mathrm{mg}$, & $0.19 \mathrm{mmol}$, & $97 \%$ & $65: 35$ & $21.159 \mathrm{~min}$ & $24.852 \mathrm{~min}$ \\
\hline 2 & B & $32.7 \mathrm{mg}$, & $0.18 \mathrm{mmol}$, & $89 \%$ & $85: 15$ & $21.489 \mathrm{~min}$ & $25.252 \mathrm{~min}$ \\
\hline
\end{tabular}

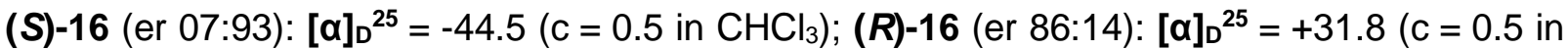
$\left.\mathrm{CHCl}_{3}\right) ; \mathbf{R}_{\boldsymbol{f}}=0.30\left(\mathrm{Et}_{2} \mathrm{O}: n\right.$-pentane, 5:95); ${ }^{1} \mathrm{H}-\mathrm{NMR}\left(400 \mathrm{MHz}, \mathrm{CDCl}_{3}\right): \delta=7.25-7.15(\mathrm{~m}, 2 \mathrm{H}$, H5), $7.08-6.98(\mathrm{~m}, 2 \mathrm{H}, \mathrm{H} 6), 4.55-4.44(\mathrm{~m}, 2 \mathrm{H}, \mathrm{H} 1), 3.63\left(\mathrm{~h},{ }^{3} \mathrm{~J}_{\mathrm{HH}}=7.1 \mathrm{~Hz}, 1 \mathrm{H}, \mathrm{H} 2\right), 1.37(\mathrm{~d}$, $\left.{ }^{3} \mathrm{~J}_{\mathrm{HH}}=7.1 \mathrm{~Hz}, 3 \mathrm{H}, \mathrm{H} 3\right) \mathrm{ppm} ;{ }^{13} \mathrm{C}\left\{{ }^{1} \mathrm{H}\right\}-\mathrm{NMR}\left(101 \mathrm{MHz}, \mathrm{CDCl}_{3}\right): \delta=162.1\left(\mathrm{~d},{ }^{1} \mathrm{~J}_{\mathrm{CF}}=267.1 \mathrm{~Hz}, \mathrm{C} 7\right)$, $136.7\left(\mathrm{~d},{ }^{4} J_{\mathrm{CF}}=3.3 \mathrm{~Hz}, \mathrm{C} 4\right), 128.6\left(\mathrm{~d},{ }^{3} J_{\mathrm{CF}}=8.1 \mathrm{~Hz}, \mathrm{C} 5\right), 116.0\left(\mathrm{~d},{ }^{2} J_{\mathrm{CF}}=21.5 \mathrm{~Hz}, \mathrm{C} 6\right), 82.0$ (C1), 38.1 (C2), 19.0 (C3) ppm; ${ }^{19} \mathrm{~F}-\left\{{ }^{1} \mathrm{H}\right\}-N M R\left(376 \mathrm{MHz}, \mathrm{CDCl}_{3}\right): \delta=-114.79$ ppm; IR (ATR): $\tilde{v}=2971(w), 2930(w), 1605(w), 1547(s), 1510(s), 1456(w), 1432(w), 1377(m), 1343(w)$, $1301(\mathrm{w}), 1223(\mathrm{~m}), 1161(\mathrm{~m}), 1118(\mathrm{w}), 1101(\mathrm{w}), 1028(\mathrm{w}), 1015(\mathrm{w}), 899(\mathrm{w}), 833(\mathrm{~s}), 778$ (w), $720(w), 697(w) \mathrm{cm}^{-1}$; GC-El-MS: $\mathrm{m} / \mathrm{z}$ : [M] ${ }^{+}$calcd. for $\mathrm{C}_{9} \mathrm{H}_{10} \mathrm{FNO}_{2}{ }^{+}$: 183.06; found: 183.03 . The analytical data are in agreement with the literature. ${ }^{[1]}$ 


\section{HPLC trace of rac-16:}

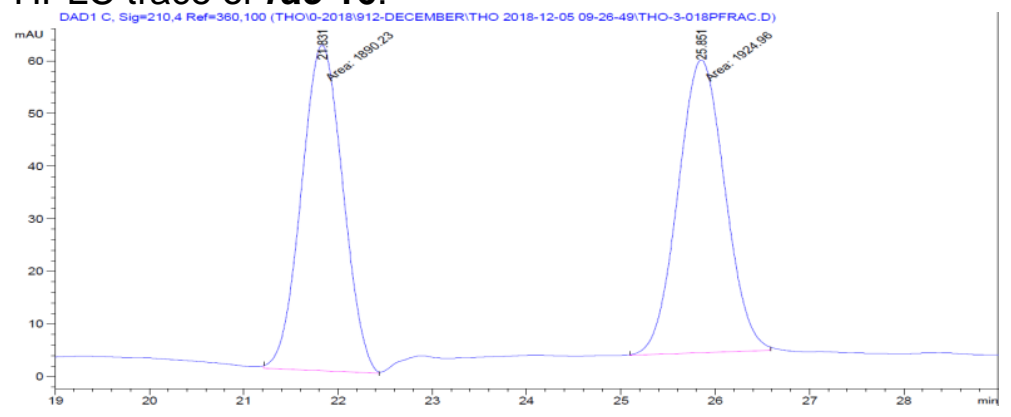

Signal 1: DAD1 C, Sig $=210,4$ Ref $=360,100$

Peak RetTime Type Width Area Height Area

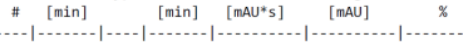

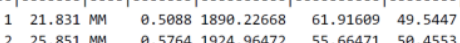

$\begin{array}{lll} & & \\ \text { Totals : } & 3815.19141 & 117.58880\end{array}$

HPLC trace of (S)-16 (Table 7, entry 1):

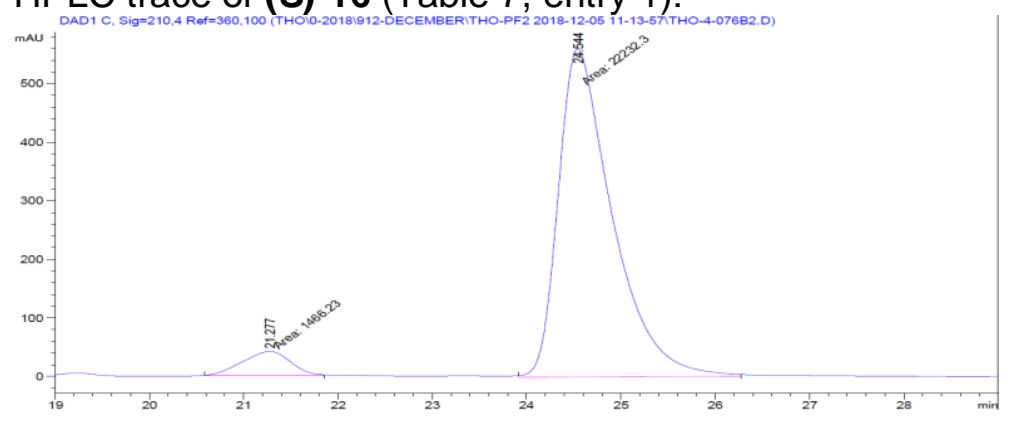

Signal 1: DAD1 C, Sig $=210,4$ Ref $=360,100$

Peak RetTime Type width Area Height Are

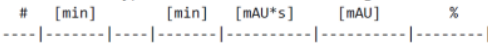

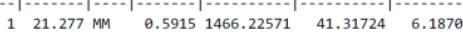

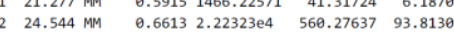

Totals :

$2.36985 e 4 \quad 601.59361$

\section{HPLC for $(\boldsymbol{R})-\mathbf{1 6}$ (Table 8, entry 2):}

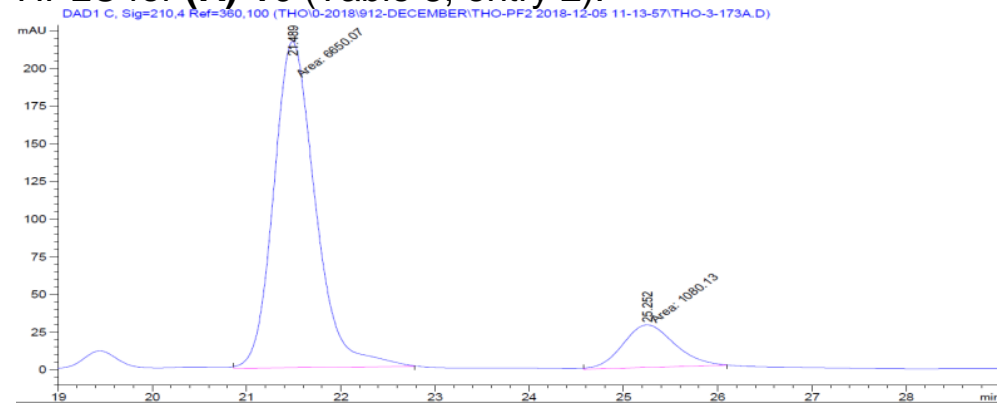

Signal 1: DAD1 C, Sig $=210,4$ Ref $=360,180$

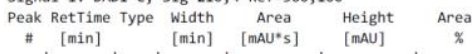

\# [min] 1 min] [mAU*s] [mAU]

$\begin{array}{lllllll}1 & 21.489 & \text { MM } & 0.5115 & 6650.06641 & 216.68570 & 86.0272\end{array}$

$\begin{array}{llllll}25.252 & \text { MM } & 0.6365 & 1080.12671 & 28.28345 & 13.9728\end{array}$

Totals :

$7730.19312 \quad 244.96915$

Figure 9: HPLC traces of 16; top: rac-16, middle: (S)-16 (Table 7, entry 1); bottom: $(\boldsymbol{R})-16$ (Table 8, entry 2). 

$2.3 \mathrm{~mL}$ ) to obtain rac-17 after $4 \mathrm{~h}$ as a colorless oil $(70.5 \mathrm{mg}$, $0.35 \mathrm{mmol}, 88 \%)$.

\section{(S)-1-Chloro-4-(1-nitropropan-2-yl)benzene ((S)-17)}

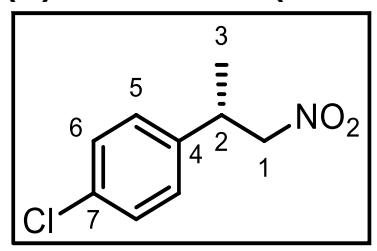

Prepared according to general procedure E, E-5 (40 mg) was converted to (S)-17, a colorless oil, using the catalyst stated in Table 9. The outcome for the given catalyst is summarized in Table 9 with the enantiomeric ratio determined by chiral HPLC (Reprosil Chiral-OM, $n$-hexane: $\mathrm{PrOH}$ 99:1, $1.0 \mathrm{~mL} / \mathrm{min}, \lambda=210 \mathrm{~nm}$ ).

Table 9: Reaction outcome for the hydrogenation of E-5 to (S)-17.

\begin{tabular}{|c|c|c|c|c|c|c|c|}
\hline entry & catalyst & yield & & & er & $t_{\mathrm{R}}($ minor $)$ & $t_{R}($ major $)$ \\
\hline $\begin{array}{l}1 \\
2\end{array}$ & $\begin{array}{l}\text { A } \\
\text { B }\end{array}$ & $\begin{array}{l}38.8 \mathrm{mg}, \\
38.1 \mathrm{mg}\end{array}$ & $\begin{array}{l}0.19 \mathrm{mmol} \text {, } \\
0.19 \mathrm{mmol}\end{array}$ & $\begin{array}{l}97 \% \\
95 \%\end{array}$ & $\begin{array}{l}\text { 05:95 } \\
11: 89\end{array}$ & $\begin{array}{l}13.753 \mathrm{~min} \\
13.910 \mathrm{~min}\end{array}$ & $\begin{array}{l}18.563 \mathrm{~min} \\
18.787 \mathrm{~min}\end{array}$ \\
\hline
\end{tabular}

(R)-1-Chloro-4-(1-nitropropan-2-yl)benzene ((R)-17)<smiles>C[C@H](c1ccc(Cl)cc1)[N+](=O)[O-]</smiles>
Prepared according to general procedure $\mathbf{F}, \mathbf{Z}-5(40 \mathrm{mg})$ was converted to $(\boldsymbol{R})-\mathbf{1 7}$, a colorless oil, using the catalyst stated in Table 10 . The outcome for the given catalyst is summarized in Table 10 with the enantiomeric ratio determined by chiral HPLC (Reprosil Chiral-OM, $n$-hexane: $\mathrm{PrOH}$ 99:1, $1.0 \mathrm{~mL} / \mathrm{min}, \lambda=210 \mathrm{~nm}$ ).

Table 10: Reaction outcome for the hydrogenation of $\mathbf{Z - 5}$ to $(\boldsymbol{R})-\mathbf{1 7}$.

\begin{tabular}{|c|c|c|c|c|c|c|c|}
\hline entry & catalyst & yield & & & er & $t_{R}$ (major) & $t_{R}($ minor $)$ \\
\hline 1 & $A$ & $36.4 \mathrm{mg}$, & $0.18 \mathrm{mmol}$, & $91 \%$ & 87:13 & $13.762 \mathrm{~min}$ & \\
\hline
\end{tabular}

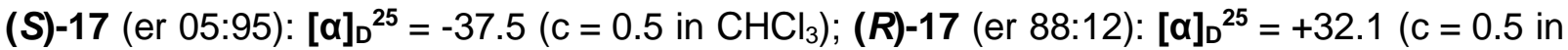
$\left.\mathrm{CHCl}_{3}\right) ; \mathbf{R}_{\boldsymbol{f}}=0.27\left(\mathrm{Et}_{2} \mathrm{O}: n\right.$-pentane, 5:95); ${ }^{1} \mathrm{H}-\mathrm{NMR}\left(600 \mathrm{MHz}, \mathrm{CDCl}_{3}\right): \delta=7.35-7.27(\mathrm{~m}, 2 \mathrm{H}$, $\mathrm{H} 6), 7.19-7.14(\mathrm{~m}, 2 \mathrm{H}, \mathrm{H} 5), 4.55-4.43(\mathrm{~m}, 2 \mathrm{H}, \mathrm{H} 1), 3.62\left(\mathrm{~h},{ }^{3} \mathrm{~J}_{\mathrm{HH}}=7.1 \mathrm{~Hz}, 1 \mathrm{H}, \mathrm{H} 2\right), 1.36(\mathrm{~d}$, $\left.{ }^{3} J_{\mathrm{HH}}=7.1 \mathrm{~Hz}, 3 \mathrm{H}, \mathrm{H} 3\right) \mathrm{ppm} ;{ }^{13} \mathrm{C}\left\{{ }^{1} \mathrm{H}\right\}-\mathrm{NMR}\left(151 \mathrm{MHz}, \mathrm{CDCl}_{3}\right): \delta=139.5(\mathrm{C} 4), 133.5$ (C7), 129.3 (C6), 128.4 (C5), 81.7 (C1), 38.2 (C2), 18.9 (C3) ppm; IR (ATR): $\tilde{v}=2974$ (w), 2934 (w), 1548 (s), $1494(\mathrm{~m}), 1456(\mathrm{w}), 1432(\mathrm{w}), 1413(\mathrm{w}), 1377(\mathrm{~m}), 1343(\mathrm{w}), 1302(\mathrm{w}), 1226(\mathrm{w}), 1199(\mathrm{w})$, 1120 (w), $1092(\mathrm{~m}), 1013(\mathrm{~m}), 900$ (w), 826 (s), 774 (w), $738(\mathrm{~m}), 717$ (w) cm-1; HR-ESI-MS: $\mathrm{m} / \mathrm{z}$ : $[\mathrm{M}-\mathrm{H}]^{+}$calcd. for $\mathrm{C}_{9} \mathrm{H}_{9} \mathrm{ClNO}_{2}{ }^{+}$: 198.0322; found: 198.73782. The analytical data are in agreement with the literature. ${ }^{[13]}$ 


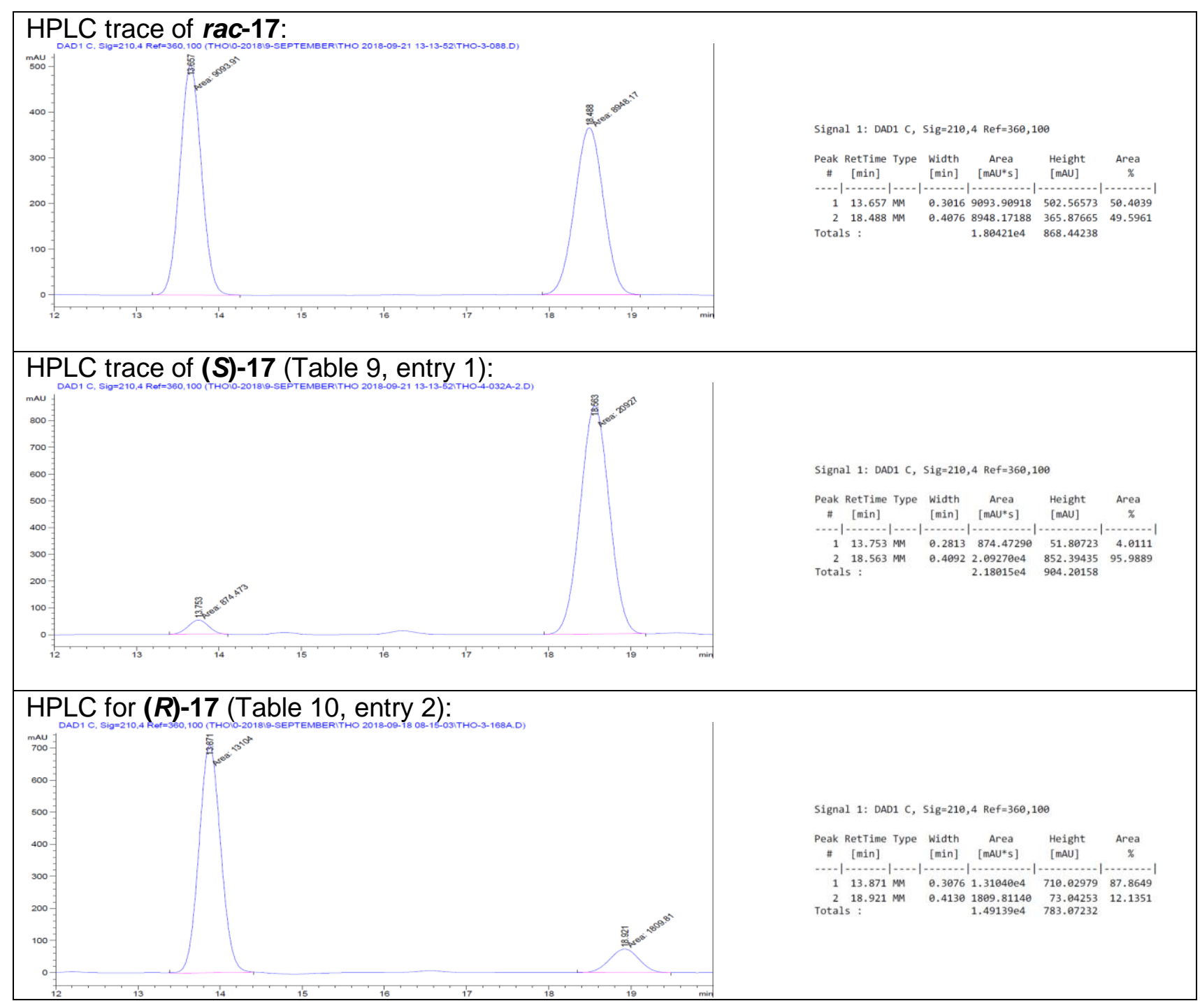

Figure 10: HPLC traces for 17; top: rac-17, middle: (S)-17 (Table 9, entry 1); bottom: $(\boldsymbol{R})$-17 (Table 10, entry 2). 


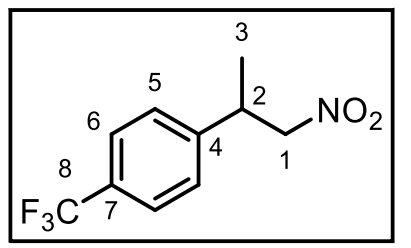
Following general procedure D, E-6 (46 mg, $0.2 \mathrm{mmol}, 1$ eq.) was treated with $\mathrm{NaBH}_{4}(11 \mathrm{mg}, 0.3 \mathrm{mmol}, 1.5$ eq.) in THF:MeOH (10:1, $1.4 \mathrm{~mL})$ to obtain rac-18 after $4 \mathrm{~h}$ as a colorless oil $(22.0 \mathrm{mg}$, $0.10 \mathrm{mmol}, 47 \%)$.

(S)-1-(1-Nitropropan-2-yl)-4-(trifluoromethyl)benzene ((S)-18)

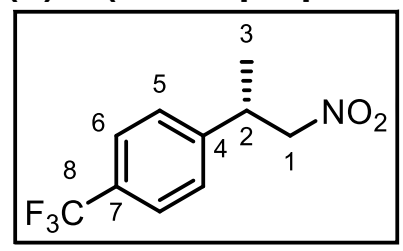

Prepared according to general procedure E, E-6 $(46 \mathrm{mg})$ was converted to (S)-18, a colorless oil, using the catalyst stated in Table 11. The outcome for the given catalyst is summarized in Table 11 with the enantiomeric ratio determined by chiral HPLC (Reprosil Chiral-OM, $n$-hexane:PrOH 97:3, $1.0 \mathrm{~mL} / \mathrm{min}, \lambda=210 \mathrm{~nm}$ ).

Table 11: Reaction outcome for the hydrogenation of $E-6$ to (S)-18.

\begin{tabular}{|c|c|c|c|c|c|c|c|}
\hline entry & catalyst & yield & & & er & $t_{R}($ minor $)$ & $t_{R}$ (major) \\
\hline $\begin{array}{l}1 \\
2\end{array}$ & $\begin{array}{l}A \\
B\end{array}$ & $\begin{array}{l}35.7 \mathrm{mg} \text {, } \\
39.0 \mathrm{mg}\end{array}$ & $\begin{array}{l}0.15 \mathrm{mmol}, \\
0.17 \mathrm{mmol}\end{array}$ & $\begin{array}{l}78 \% \\
84 \%\end{array}$ & $\begin{array}{l}10: 90 \\
12: 88\end{array}$ & & $\begin{array}{l}11.817 \mathrm{~min} \\
12.006 \mathrm{~min}\end{array}$ \\
\hline
\end{tabular}

(R)-1-(1-Nitropropan-2-yl)-4-(trifluoromethyl)benzene (( $R)$-18)<smiles>O=[N+]([O-])C(CC1CC2CCCC1C2)c1ccc(F)cc1</smiles>
Prepared according to general procedure F, Z-6 (46 mg) was converted to $(R)-18$, a colorless oil, using the catalyst stated in Table 12. The outcome for the given catalyst is summarized in Table 12 with the enantiomeric ratio determined by chiral HPLC Reprosil Chiral-OM, $n$-hexane:(PrOH 97:3, $1.0 \mathrm{~mL} / \mathrm{min}, \lambda=210 \mathrm{~nm}$ ).

Table 12: Reaction outcome for the hydrogenation of Z-18 to $(\boldsymbol{R}) \mathbf{- 1 8}$.

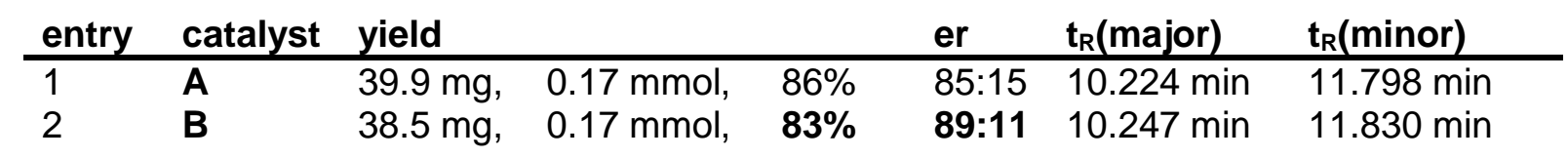

(S)-18 (er 10:90): $[\alpha]_{\mathrm{D}}{ }^{25}=-25.1\left(\mathrm{c}=0.5\right.$ in $\left.\mathrm{CHCl}_{3}\right) ;(\boldsymbol{R})-\mathbf{1 8}(\mathrm{er} 89: 11)$ : $[\alpha]_{\mathrm{D}}{ }^{25}=+30.0(\mathrm{c}=0.5$ in $\left.\mathrm{CHCl}_{3}\right) ; \mathbf{R}_{\boldsymbol{f}}=0.19\left(\mathrm{Et}_{2} \mathrm{O}: n\right.$-pentane, $\left.5: 95\right) ;{ }^{1} \mathrm{H}-\mathrm{NMR}\left(600 \mathrm{MHz}, \mathrm{CDCl}_{3}\right): \delta=7.61\left(\mathrm{~d},{ }^{3} \mathrm{~J}_{\mathrm{HH}}=8.1 \mathrm{~Hz}\right.$, $2 \mathrm{H}, \mathrm{H} 6), 7.36\left(\mathrm{~d},{ }^{3} \mathrm{JHH}_{\mathrm{HH}}=8.1 \mathrm{~Hz}, 2 \mathrm{H}, \mathrm{H} 5\right), 4.61-4.48(\mathrm{~m}, 2 \mathrm{H}, \mathrm{H} 1), 3.72\left(\mathrm{sext},{ }^{3} \mathrm{~J}_{\mathrm{HH}}=7.2 \mathrm{~Hz}, 1 \mathrm{H}\right.$, $\mathrm{H} 2), 1.41\left(\mathrm{~d},{ }^{3} \mathrm{JHH}_{\mathrm{HH}}=7.2 \mathrm{~Hz}, 3 \mathrm{H}, \mathrm{H} 3\right) \mathrm{ppm} ;{ }^{13} \mathrm{C}\left\{{ }^{1} \mathrm{H}\right\}-\mathrm{NMR}\left(101 \mathrm{MHz}, \mathrm{CDCl}_{3}\right): \delta=145.1$ (q, ${ }^{5} \mathrm{~J}_{\mathrm{CF}}=$ $1.2 \mathrm{~Hz}, \mathrm{C} 4), 130.1$ (q, $\left.{ }^{2} \mathrm{~J}_{\mathrm{CF}}=32.6 \mathrm{~Hz}, \mathrm{C} 7\right), 127.5(\mathrm{C} 5), 126.1$ (q, $\left.{ }^{3} \mathrm{~J}_{\mathrm{CF}}=3.8 \mathrm{~Hz}, \mathrm{C} 6\right), 124.1$ (q, $\left.{ }^{1} \mathrm{~J}_{\mathrm{CF}}=272.1 \mathrm{~Hz}, \mathrm{C} 8\right), 81.4(\mathrm{C} 1), 38.6$ (C2), 18.9 (C3) ppm; ${ }^{9} \mathrm{~F}-\mathrm{NMR}\left(376 \mathrm{MHz}, \mathrm{CDCl}_{3}\right)$ : $\delta=-62.63$ (s) ppm; IR (ATR): $\tilde{v}=2976$ (w), 2939 (w), 1621 (w), 1551 (s), 1459 (w), $1442(w)$, $1378(w), 1323(\mathrm{~s}), 1164(\mathrm{~m}), 1119(\mathrm{~s}), 1068(\mathrm{~s}), 1016(\mathrm{~m}), 956(\mathrm{w}), 901(\mathrm{w}), 839(\mathrm{w}), 768(\mathrm{w})$,

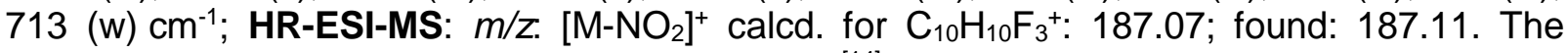
analytical data are in agreement with the literature. ${ }^{[14]}$ 


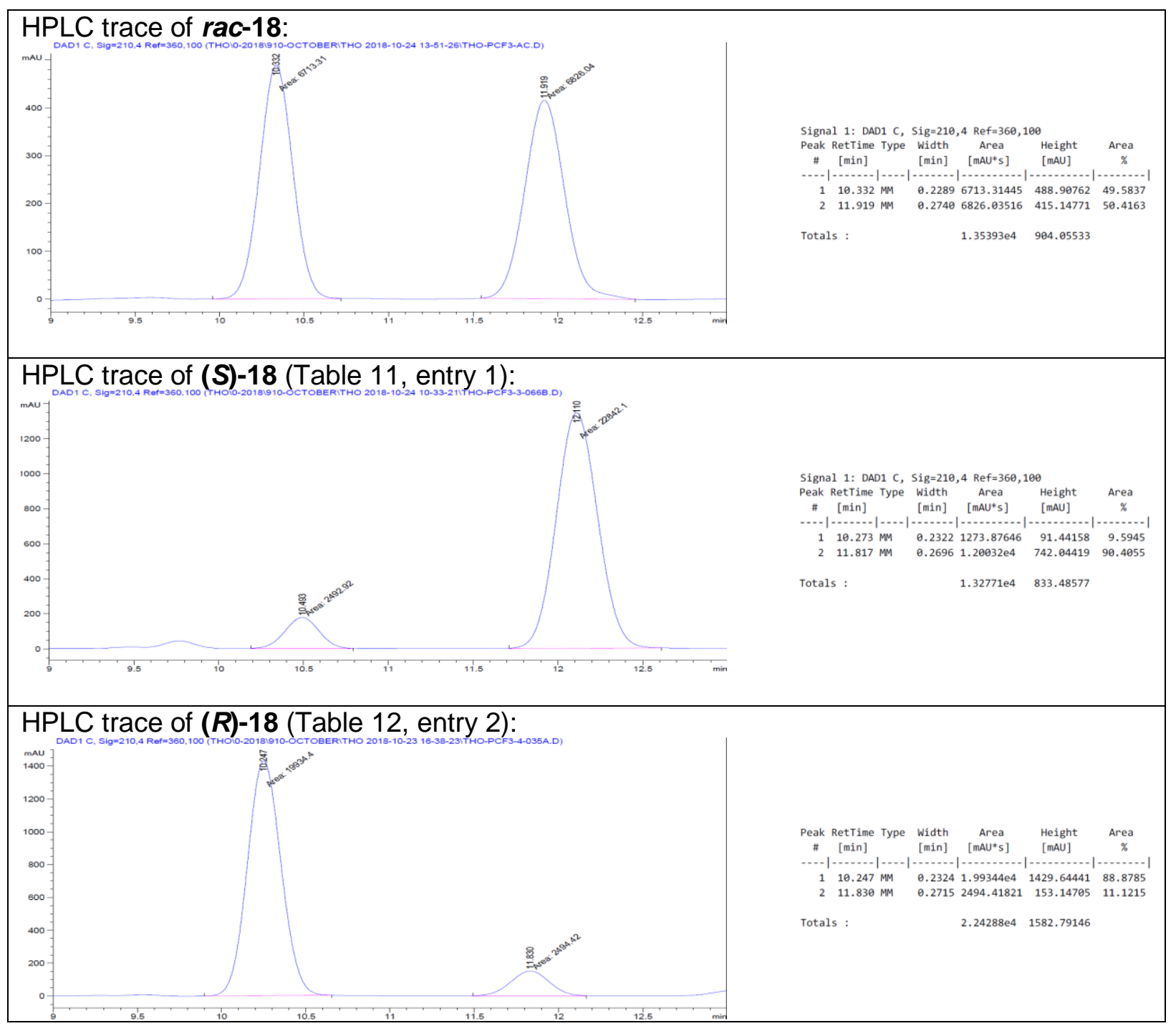

Figure 11: HPLC traces of 18; top: rac-18, middle: (S)-18 (Table 11, entry 1); bottom: $(\boldsymbol{R})$-18 (Table 12, entry 2). 


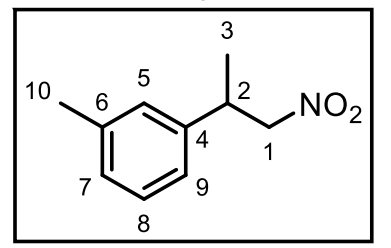

Following general procedure D, $E-8(89 \mathrm{mg}, 0.5 \mathrm{mmol}$, 1 eq.) was treated with $\mathrm{NaBH}_{4}(28 \mathrm{mg}, 0.75 \mathrm{mmol}, 1.5$ eq.) in THF:MeOH (10:1, $4 \mathrm{~mL}$ ) to obtain rac-19 after $4 \mathrm{~h}$ as a colorless oil $(58.5 \mathrm{mg}, 0.33 \mathrm{mmol}$, $65 \%)$.

\section{(S)-1-Methyl-3-(1-nitropropan-2-yl)benzene ((S)-19)}

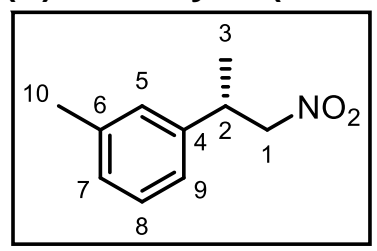

Prepared according to general procedure E, E-8 (35 mg) was converted to (S)-19, a colorless oil, using the catalyst stated in Table 13. The outcome for the given catalyst is summarized in Table 13 with the enantiomeric ratio determined by chiral HPLC (Reprosil ChiralOM, $n$-hexane: $\mathrm{PrOH}$ 97:3, $1.0 \mathrm{~mL} / \mathrm{min}, \lambda=210 \mathrm{~nm}$ ).

Table 13: Reaction outcome for the hydrogenation of $E-8$ to (S)-19.

\begin{tabular}{|c|c|c|c|c|c|c|c|}
\hline entry & catalyst & yield & & & er & $t_{R}($ minor $)$ & $t_{R}($ major $)$ \\
\hline 1 & A & $33.3 \mathrm{mg}$, & $0.19 \mathrm{mmol}$, & $93 \%$ & 05:95 & $7.708 \mathrm{~min}$ & $9 \min$ \\
\hline
\end{tabular}

(R)-1-Methyl-3-(1-nitropropan-2-yl)benzene $((R)$-19)

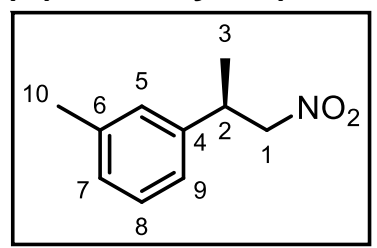

Prepared according to general procedure F, Z-8 $(35 \mathrm{mg})$ was converted to $(\boldsymbol{R})-\mathbf{1 9}$, a colorless oil, using the catalyst stated in Table 14 . The outcome for the given catalyst is summarized in Table 14 with the enantiomeric ratio determined by chiral HPLC Reprosil Chiral-OM, $n$-hexane: $\mathrm{PrOH}$ 97:3, $1.0 \mathrm{~mL} / \mathrm{min}, \lambda=210 \mathrm{~nm}$ ).

Table 14: Reaction outcome for the hydrogenation of $\mathbf{Z - 8}$ to $(\boldsymbol{R})-\mathbf{1 9}$.

\begin{tabular}{|c|c|c|c|c|c|c|c|}
\hline entry & catalyst & yield & & & er & $t_{R}$ (major) & $t_{R}($ minor $)$ \\
\hline $\begin{array}{l}1 \\
2\end{array}$ & $\begin{array}{l}A \\
B\end{array}$ & $\begin{array}{l}26.3 \mathrm{mg}, \\
29.0 \mathrm{mg},\end{array}$ & $\begin{array}{l}0.15 \mathrm{mmol} \text {, } \\
0.16 \mathrm{mmol}\end{array}$ & $\begin{array}{l}73 \% \\
81 \%\end{array}$ & $\begin{array}{l}87: 13 \\
91: 09\end{array}$ & $\begin{array}{l}7.372 \mathrm{~min} \\
7.648 \mathrm{~min}\end{array}$ & $\begin{array}{l}10.129 \mathrm{~min} \\
10.087 \mathrm{~min}\end{array}$ \\
\hline
\end{tabular}

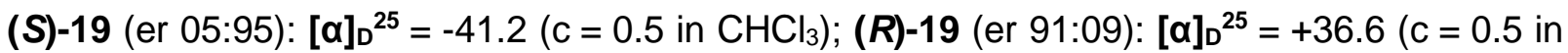
$\left.\mathrm{CHCl}_{3}\right) ; \mathbf{R}_{\boldsymbol{f}}=\left(\mathrm{Et}_{2} \mathrm{O}: n\right.$-pentane, 5:95); ${ }^{1} \mathrm{H}-\mathrm{NMR}\left(600 \mathrm{MHz}, \mathrm{CDCl}_{3}\right): \delta=7.49-7.42(\mathrm{~m}, 1 \mathrm{H}, \mathrm{H} 8)$, $7.31\left(\mathrm{~d},{ }^{3} \mathrm{~J}_{\mathrm{HH}}=7.8 \mathrm{~Hz}, 1 \mathrm{H}, \mathrm{H} 7\right), 7.27-7.23(\mathrm{~m}, 2 \mathrm{H}, \mathrm{H} 5, \mathrm{H} 9), 4.81-4.66(\mathrm{~m}, 2 \mathrm{H}, \mathrm{H} 1), 3.89-$ $3.75(\mathrm{~m}, 1 \mathrm{H}, \mathrm{H} 2), 2.57(\mathrm{~s}, 3 \mathrm{H}, \mathrm{H} 10), 1.59\left(\mathrm{~d},{ }^{3} \mathrm{JHH}_{\mathrm{HH}}=7.0 \mathrm{~Hz}, 3 \mathrm{H}, \mathrm{H} 3\right) \mathrm{ppm} ;{ }^{3} \mathbf{C}\left\{{ }^{1} \mathrm{H}\right\}-\mathbf{N M R}(151$ $\mathrm{MHz}, \mathrm{CDCl}_{3}$ ): $\delta=141.0$ (C4), 138.8 (C6), 129.0 (C8), 128.5 (C7), 127.8 (C5), 124.0 (C9), 82.1 (C1), 38.7 (C2), 21.6 (C10), 18.9 (C3) ppm; IR (ATR): $\tilde{v}=2973$ (w), 2922 (w), 1608 (w), 1547 (s), $1491(w), 1456(w), 1431(w), 1375(m), 1212(w), 1166(w), 1119$ (w), $1040(w), 904(w)$, $882(\mathrm{w}), 786(\mathrm{~m}), 703$ (s) cm${ }^{-1}$; GC-El-MS: $\mathrm{m} / \mathrm{z}:[\mathrm{M}-\mathrm{H}]^{+}$calcd. for $\mathrm{C}_{10} \mathrm{H}_{12} \mathrm{NO}_{2}{ }^{+}:$178.08626; found: 178.08632 . 


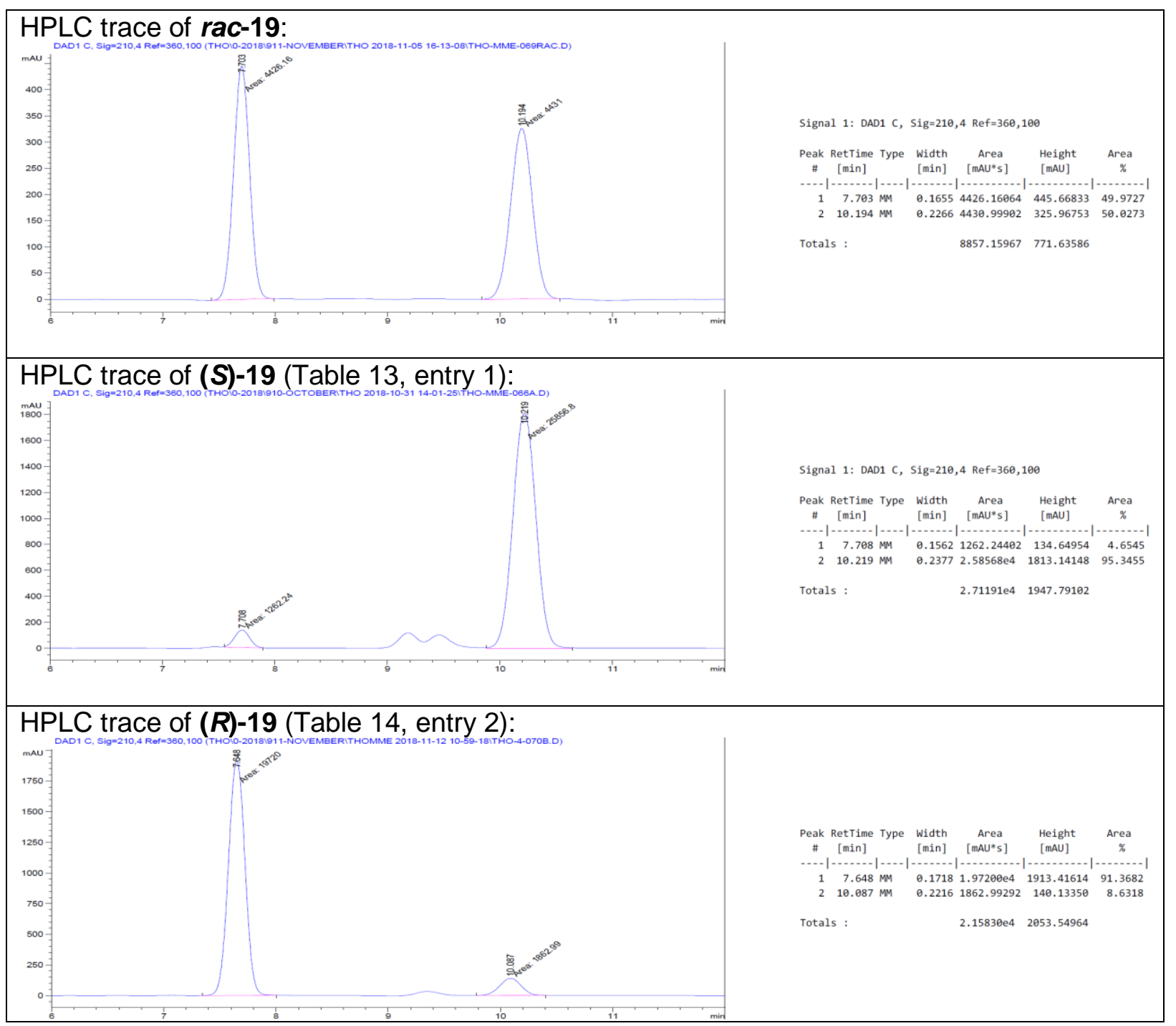

Figure 12: HPLC traces for 19; top: rac-19, middle: (S)-19 (Table 13, entry 1); bottom: $(\boldsymbol{R})$-19 (Table 14, entry 2). 
rac-1-Chloro-3-(1-nitropropan-2-yl)benzene (rac-20)

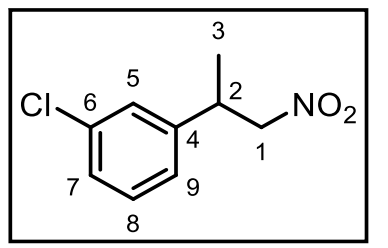

Following general procedure $\mathbf{D}, \boldsymbol{E}-\mathbf{9}(299 \mathrm{mg}, 1.5 \mathrm{mmol}, 1$ eq.) was treated with $\mathrm{NaBH}_{4}$ (85 mg, $2.3 \mathrm{mmol}$, 1.5 eq.) in THF:MeOH (10:1, $7.5 \mathrm{~mL}$ ) to obtain rac-20 after $4 \mathrm{~h}$ as a colorless oil $(266.2 \mathrm{mg}$, $1.33 \mathrm{mmol}, 89 \%)$.

\section{(S)-1-Chloro-3-(1-nitropropan-2-yl)benzene ((S)-20)}

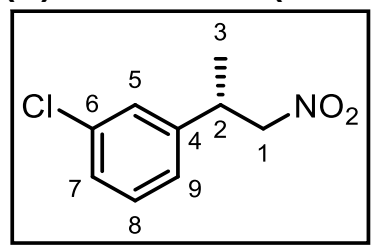

Prepared according to general procedure E, E-9 (40 mg) was converted to (S)-20, a colorless oil, using the catalyst stated in Table 15. The outcome for the given catalyst is summarized in Table 15 with the enantiomeric ratio determined by chiral HPLC (Reprosil ChiralOM, $n$-hexane: $\mathrm{PrOH}$ 97:3, $1.0 \mathrm{~mL} / \mathrm{min}, \lambda=210 \mathrm{~nm}$ ).

Table 15: Reaction outcome for the hydrogenation of $E-9$ to $(S)-20$.

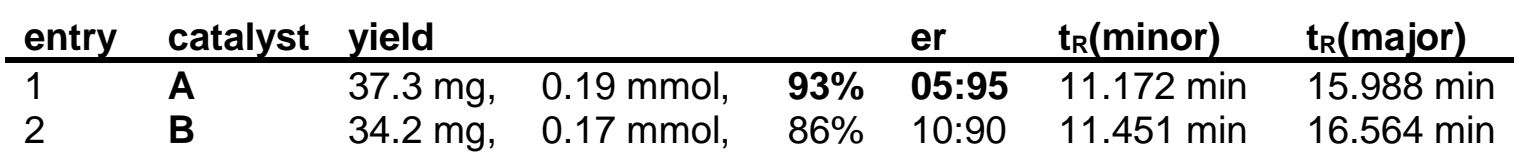

\section{(R)-1-Chloro-3-(1-nitropropan-2-yl)benzene $((R)$-20)}<smiles>C[C@H](c1cccc(Cl)c1)[N+](=O)[O-]</smiles>
Prepared according to general procedure $\mathbf{F}, \mathbf{Z}-\mathbf{9}(40 \mathrm{mg})$ was converted to $(\boldsymbol{R})-\mathbf{2 0}$, a colorless oil, using the catalyst stated in Table 16 . The outcome for the given catalyst is summarized in Table 16 with the enantiomeric ratio determined by chiral HPLC (Reprosil Chiral-OM, $n$-hexane:PrOH 97:3, $1.0 \mathrm{~mL} / \mathrm{min}, \lambda=210 \mathrm{~nm}$ ).

Table 16: Reaction outcome for the hydrogenation of $\mathbf{Z}-9$ to $(\boldsymbol{R})-20$.

\begin{tabular}{llllllll} 
entry & catalyst & yield & & & er & $\mathbf{t}_{\mathbf{R}}$ (major) & $\mathbf{t}_{\mathbf{R}}$ (minor) \\
\hline 1 & A & $28.5 \mathrm{mg}$, & $0.14 \mathrm{mmol}$, & $71 \%$ & $78: 22$ & $11.532 \mathrm{~min}$ & $16.756 \mathrm{~min}$ \\
2 & B & $26.8 \mathrm{mg}$, & $0.13 \mathrm{mmol}$, & $\mathbf{6 7 \%}$ & $\mathbf{9 3 : 0 7}$ & $11.401 \mathrm{~min}$ & $16.545 \mathrm{~min}$
\end{tabular}

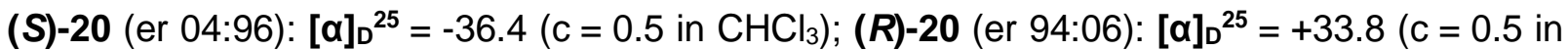
$\left.\mathrm{CHCl}_{3}\right) ; \mathbf{R}_{\boldsymbol{f}}=0.33\left(\mathrm{Et}_{2} \mathrm{O}: n\right.$-pentane, 5:95); ${ }^{1} \mathrm{H}-\mathrm{NMR}\left(600 \mathrm{MHz}, \mathrm{CDCl}_{3}\right): \delta=7.31-7.23(\mathrm{~m}, 2 \mathrm{H}$, $\mathrm{H} 7, \mathrm{H} 8), 7.23-7.21(\mathrm{~m}, 1 \mathrm{H}, \mathrm{H} 5), 7.14-7.09(\mathrm{~m}, 1 \mathrm{H}, \mathrm{H} 9), 4.57-4.44(\mathrm{~m}, 2 \mathrm{H}, \mathrm{H} 1), 3.62(\mathrm{~h}$, $\left.{ }^{3} J_{\mathrm{HH}}=7.2 \mathrm{~Hz}, 1 \mathrm{H}, \mathrm{H} 2\right), 1.37\left(\mathrm{~d},{ }^{3} \mathrm{~J}_{\mathrm{HH}}=7.2 \mathrm{~Hz}, 3 \mathrm{H}, \mathrm{H} 3\right) \mathrm{ppm} ;{ }^{13} \mathbf{C}\left\{{ }^{1} \mathrm{H}\right\}-\mathrm{NMR}\left(151 \mathrm{MHz}, \mathrm{CDCl}_{3}\right)$ : $\delta=143.0$ (C4), 134.9 (C6), 130.4 (C8), 128.0 (C7), 127.3 (C5), 125.3 (C9), 81.5 (C1), 38.5 (C2), 18.8 (C3) ppm; IR (ATR): $\tilde{v}=2974(\mathrm{w}), 2930$ (w), 1598 (w), $1547(\mathrm{~s}), 1479(\mathrm{w}), 1456(\mathrm{~m}), 1432$ $(\mathrm{m}), 1375(\mathrm{~m}), 1346(\mathrm{w}), 1265(\mathrm{w}), 1120(\mathrm{w}), 1084(\mathrm{~m}), 1039(\mathrm{w}), 999(\mathrm{w}), 881(\mathrm{w}), 815(\mathrm{w}), 786$ (s), 738 (w), 694 (s), 677 (m) cm ${ }^{-1}$; HR-ESI-MS: $m / z$ : [M-O] ${ }^{+}$calcd. for $\mathrm{C}_{9} \mathrm{H}_{10} \mathrm{CINO}^{+}:$183.04454; found: 183.04460 . The analytical data are in agreement with the literature. ${ }^{[15]}$ 


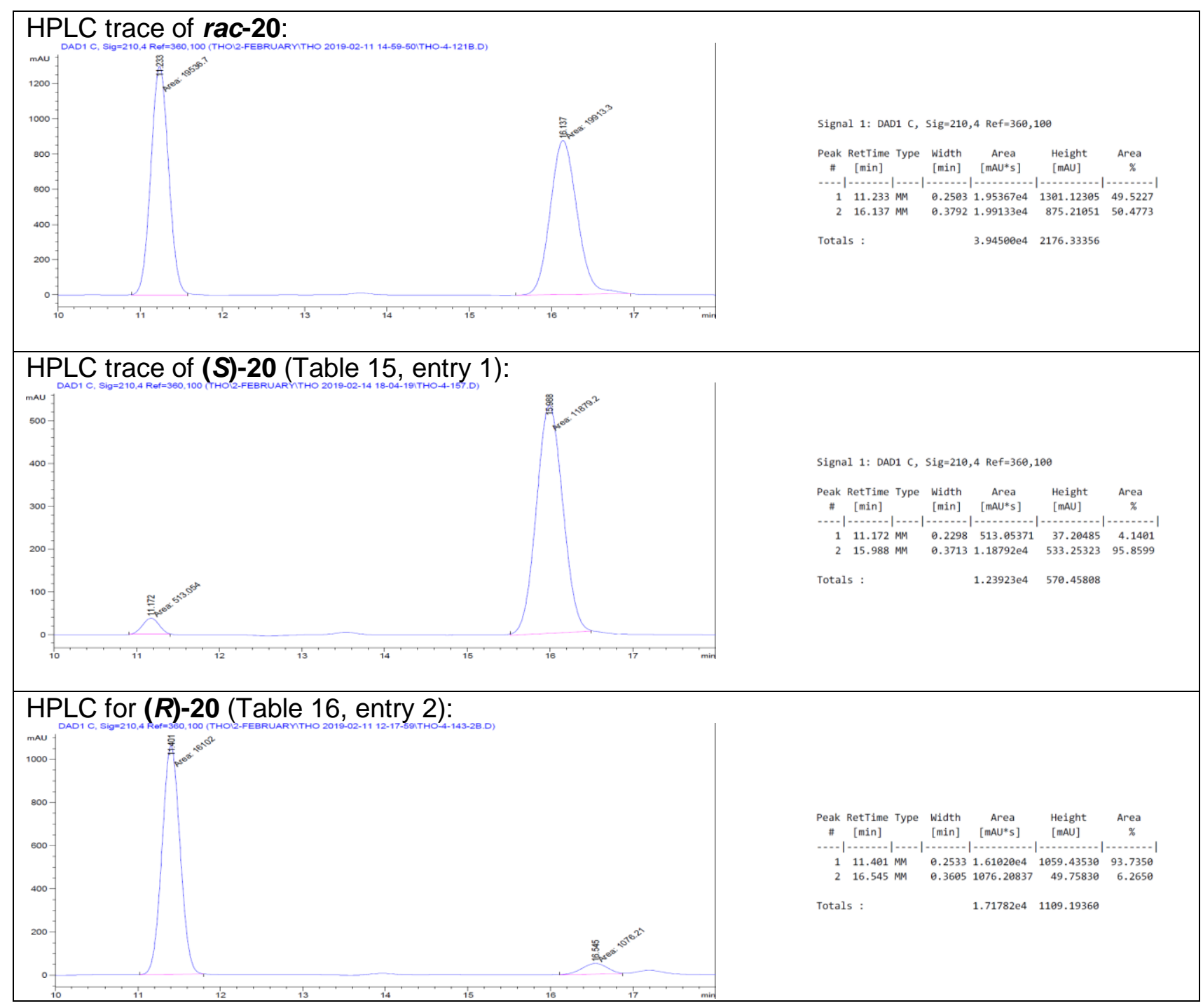

Figure 13: HPLC traces of 20; top: rac-20, middle: (S)-20 (Table 15, entry 1); bottom: $(\boldsymbol{R})-20$ (Table 16, entry 2). 


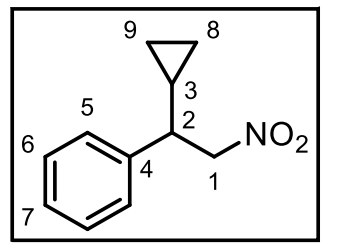

Following general procedure $\mathbf{D}, \boldsymbol{E}-10(34 \mathrm{mg}, 0.18 \mathrm{mmol}$, 1 eq.) was treated with $\mathrm{NaBH}_{4}$ (13 mg, $0.34 \mathrm{mmol}, 1.5$ eq.) in THF:MeOH (10:1, $2 \mathrm{~mL}$ ) to obtain rac-21 after $5 \mathrm{~h}$ as a colorless oil $(25.1 \mathrm{mg}, 0.13 \mathrm{mmol}$, $73 \%)$.

\section{(S)-(1-Cyclopropyl-2-nitroethyl)benzene ((S)-21)}

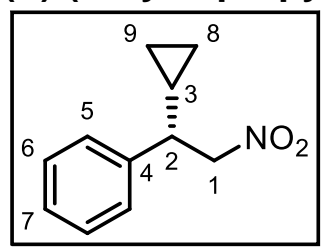

Prepared according to general procedure E, E-10 (38 mg) was converted to (S)-21, a colorless oil, using the catalyst stated in Table 17. The outcome for the given catalyst is summarized in Table 17 with the enantiomeric ratio determined by chiral HPLC (Reprosil Chiral-OM, n-hexane:PrOH 99:1, $1.0 \mathrm{~mL} / \mathrm{min}, \lambda=210 \mathrm{~nm}$ ).

Table 17: Reaction outcome for the hydrogenation of $\mathbf{E - 2 0}$ to (S)-21.

\begin{tabular}{|c|c|c|c|c|c|c|c|}
\hline entry & catalyst & yield & & & er & $t_{R}($ minor $)$ & $t_{R}$ (major) \\
\hline 1 & $A$ & $29.2 \mathrm{mg}$, & $0.15 \mathrm{mmol}$, & $76 \%$ & 03:97 & $16.587 \mathrm{~min}$ & \\
\hline 2 & B & $28.3 \mathrm{mg}$, & $0.15 \mathrm{mmol}$, & $74 \%$ & 07:93 & $16.571 \mathrm{~min}$ & $21.318 \mathrm{~min}$ \\
\hline
\end{tabular}

(R) -(1-Cyclopropyl-2-nitroethyl)benzene ((R)-21)

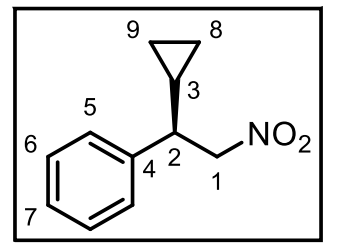

Prepared according to general procedure $\mathbf{F}, \mathbf{Z}-20(38 \mathrm{mg})$ was converted to $(\boldsymbol{R})-\mathbf{2 1}$, a colorless oil, using the catalyst stated in Table 18. The outcome for the given catalyst is summarized in Table 18 with the enantiomeric ratio determined by chiral HPLC (Reprosil Chiral-OM, $n$-hexane:PrOH 99:1, $1.0 \mathrm{~mL} / \mathrm{min}, \lambda=210 \mathrm{~nm}$ ).

Table 18: Reaction outcome for the hydrogenation of $\mathbf{Z - 1 0}$ to $(\boldsymbol{R})-\mathbf{2 1}$.

\begin{tabular}{|c|c|c|c|c|c|c|c|}
\hline entry & catalyst & yield & & & er & $t_{R}($ minor $)$ & $t_{R}$ (major) \\
\hline 1 & A & $25.7 \mathrm{mg}$ & $0.13 \mathrm{mmol}$, & $68 \%$ & 91:09 & $16.417 \mathrm{~min}$ & $21.312 \mathrm{~min}$ \\
\hline
\end{tabular}

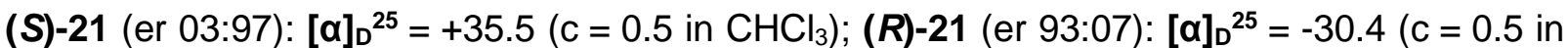
$\left.\mathrm{CHCl}_{3}\right) ; \mathbf{R}_{\boldsymbol{f}}=0.62\left(\mathrm{Et}_{2} \mathrm{O}: n\right.$-pentane 5:95); ${ }^{1} \mathrm{H}-\mathrm{NMR}\left(500 \mathrm{MHz}, \mathrm{CDCl}_{3}\right): \delta=7.37-7.32(\mathrm{~m}, 2 \mathrm{H}$, $\mathrm{H} 6), 7.30-7.22(\mathrm{~m}, 3 \mathrm{H}, \mathrm{H} 5, \mathrm{H} 7), 4.77-4.55(\mathrm{~m}, 2 \mathrm{H}, \mathrm{H} 1), 2.77-2.68(\mathrm{~m}, 1 \mathrm{H}, \mathrm{H} 2), 1.14-1.05$ (m, $1 \mathrm{H}, \mathrm{H} 3), 0.72-0.63\left(\mathrm{~m}, 1 \mathrm{H}, \mathrm{H} 8 / 8^{\prime} / 9 / 9^{\prime}\right), 0.55-0.46\left(\mathrm{~m}, 1 \mathrm{H}, \mathrm{H} 8 / 8^{\prime} / 9 / 9^{\prime}\right), 0.38-0.30(\mathrm{~m}, 1 \mathrm{H}$, $\left.\mathrm{H} 8 / 8^{\prime} / 9 / 9^{\prime}\right), 0.24-0.15$ (m, $\left.1 \mathrm{H}, \mathrm{H} 8 / 8^{\prime} / 9 / 9^{\prime}\right) \mathrm{ppm} ;{ }^{13} \mathbf{C}\left\{{ }^{1} \mathrm{H}\right\}-N M R\left(126 \mathrm{MHz}, \mathrm{CDCl}_{3}\right): \delta=139.9$ (C4), 129.0 (C6), 127.7 (C7), 127.5 (C5), 81.0 (C1), 49.4 (C2), 14.6 (C3), 5.4 (C8/9), 3.6 (C8/9) ppm; IR (ATR): v = 3083 (w), 3006 (w), 2917 (w), 1602 (w), 1547 (s), 1495 (w), 1455 (w), 1431 (w), $1377(\mathrm{~m}), 1260(\mathrm{w}), 1194(\mathrm{w}), 1145(\mathrm{w}), 1078(\mathrm{w}), 1051(\mathrm{w}), 1022(\mathrm{~m}), 987(\mathrm{w}), 946$ (w), 925 (w), 861 (w), 824 (w), 771 (w), 753 (m), 698 (s) cm ${ }^{-1}$; GC-El-MS: m/z: [M-NO2] $]^{+}$calcd. for $\mathrm{C}_{11} \mathrm{H}_{13}{ }^{+}:$: 145.10; found: 145.12 . 


\section{HPLC trace of rac-21:}

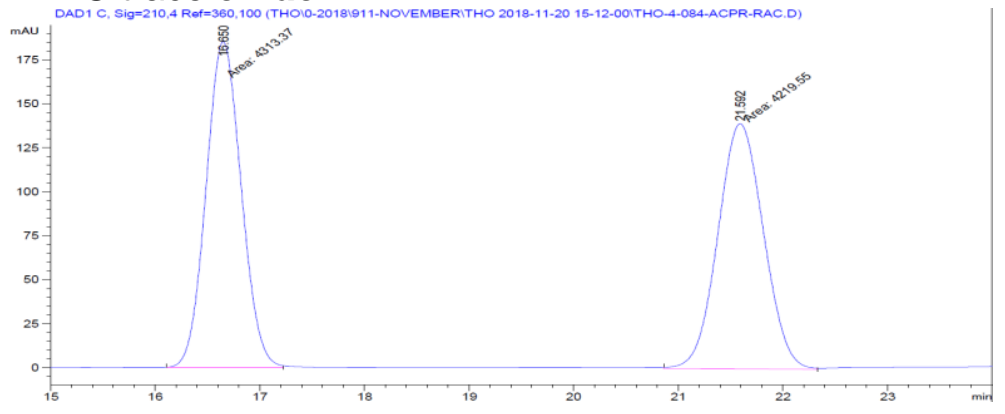

Signal 1: DAD1 C, Sig $=210,4$ Ref $=360,100$

Peak RetTime Type width Area Height Area \# [min] $[\mathrm{min}]\left[\mathrm{mAU}^{*} \mathrm{~s}\right][\mathrm{mAU}]$

$\begin{array}{llllll}1 & 16.650 \mathrm{Mm} & 0.3886 & 4313.37061 & 185.00455 & 50.5498\end{array}$ $\begin{array}{lllllll}2 & 21.592 \mathrm{MM} & 0.5847 & 4219.55029 & 139.34412 & 49.4502\end{array}$

Totals :

$8532.92990 \quad 324.34866$

\section{HPLC trace of (S)-21 (Table 17, entry 1):}

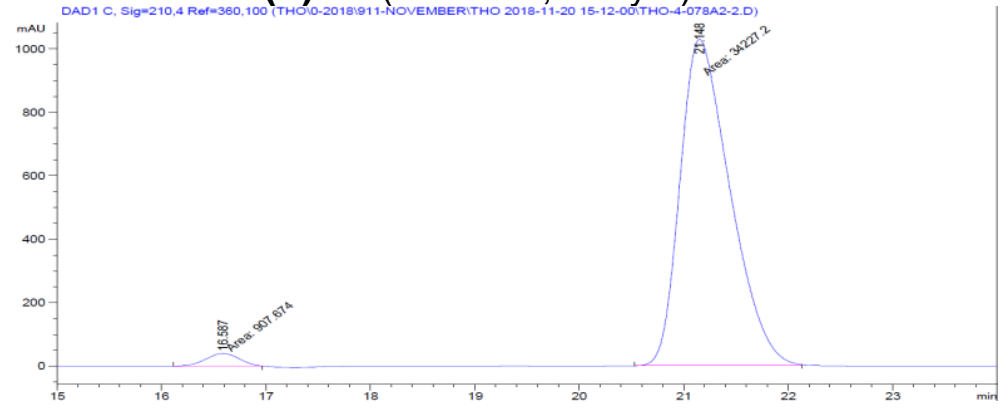

Signal 1: DAD1 C, Sig $=210,4$ Ref $=360,100$

Peak RetTime Type Width Area Height Are

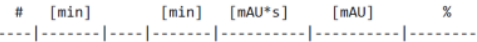

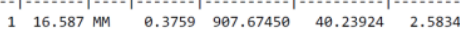

$\begin{array}{llllll}21.148 & M M & 0.5543 & 3.4227224 & 1029.23206 & 97.4166\end{array}$

Totals:

$3.51349 \mathrm{e} 4 \quad 1069.47130$

\section{HPLC trace of $(\boldsymbol{R})-21$ (Table 18, entry 2):}

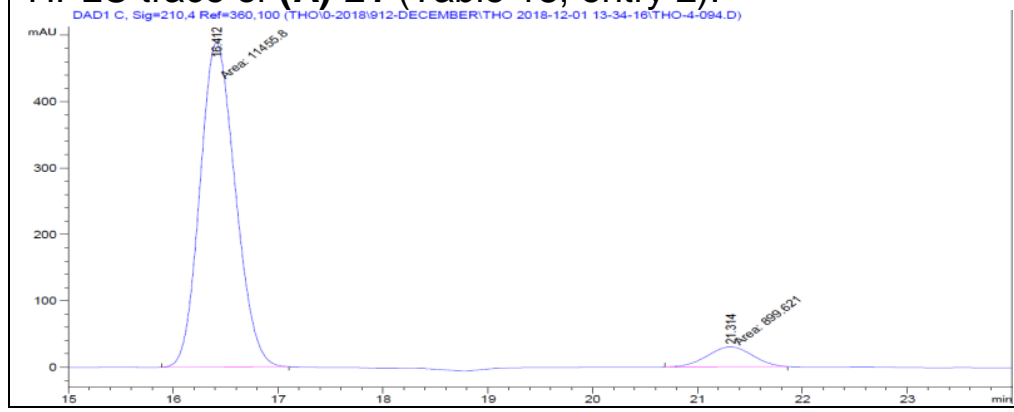

Signal 1: DAD1 C, Sig $=210,4$ Ref $=360,100$

Peak RetTime Type width Area Height Area
\# [min]

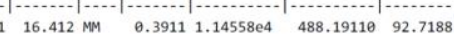

$\begin{array}{lllllll}2 & 21.314 & \text { MM } & 0.4996 & 899.62061 & 30.01181 & 7.2812\end{array}$

Totals :

$1.23554 \mathrm{e} 4 \quad 518.28291$

Figure 14: HPLC traces of 21; top: rac-21, middle: (S)-21 (Table 17, entry 1); bottom: $(\boldsymbol{R})-21$ (Table 18, entry 2). 


\section{Synthesis of thiourea catalysts}<smiles>CC(C)[C@H](NC(=O)OC(C)(C)C)C(=O)O</smiles><smiles>CC(C)(C)C1(C)CCCCC1</smiles>

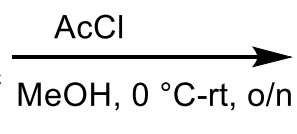

s3<smiles>CC(C)[C@H](N)C(=O)N(C)CCl</smiles>

S4

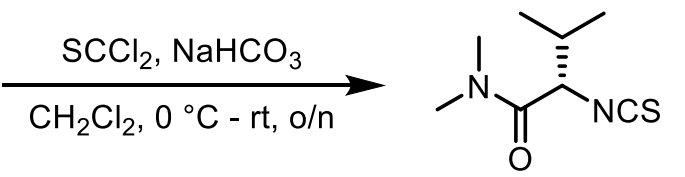

S5

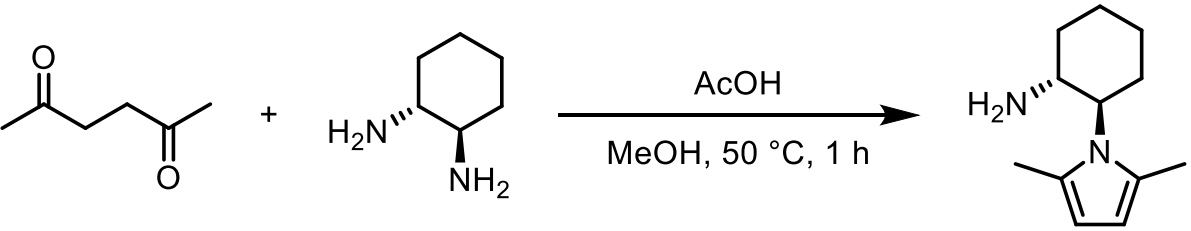

S6<smiles>CC(C)[C@H](NC(=O)[O-])C(=O)N(C)C</smiles>

S5<smiles>Cc1ccc(C)n1C1CCCC[C@H]1N</smiles>

S6

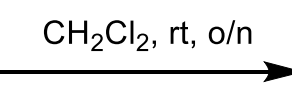

A<smiles>Cc1ccc(C)n1C1CCCC[C@H]1NC(=S)N[C@H](C(=O)N(C)C)C(C)C</smiles><smiles>CC(C)[C@H](N=CCl)C(=O)N(C)C</smiles>

S4

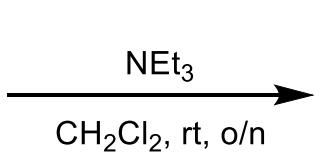

$\mathrm{CH}_{2} \mathrm{Cl}_{2}$, rt, o/n<smiles>CC(C)[C@H](NC(=S)Nc1cc(C(F)(F)F)cc(C(F)(F)F)c1)C(=O)N(C)C</smiles>

B

Scheme 1: Reaction Scheme for the preparation of the catalysts A and B.

tert-Butyl (S)-(1-(dimethylamino)-3-methyl-1-oxobutan-2-yl)carbamate (S3)

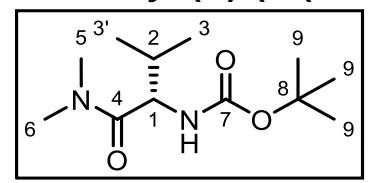

Following an adapted literature procedure ${ }^{[16]} \mathrm{N}$-Boc-L-Valine $(4.26 \mathrm{~g}$, $19.6 \mathrm{mmol}, 1$ eq.) was dissolved in dry $\mathrm{CH}_{2} \mathrm{Cl}_{2}(20 \mathrm{~mL})$ under an argon atmosphere and cooled to $0^{\circ} \mathrm{C}$ before $\mathrm{N}, \mathrm{N}$-dicyclohexyl carbodiimide (4.65 g, $22.6 \mathrm{mmol}, 1.15$ eq.) was added portionwise. $\mathrm{HNMe}_{2}(2 \mathrm{M}$ in THF, $19.6 \mathrm{~mL}, 39.2 \mathrm{mmol}, 2$ eq.) was added at $0^{\circ} \mathrm{C}$ and the reaction mixture was stirred over night while warming to room temperature. All volatiles were removed in vacuo and the crude product was purified by column chromatography $\left(\mathrm{SiO}_{2}, \mathrm{Et}_{2} \mathrm{O}: n\right.$-pentane $2: 3)$ yielding $\mathbf{S} 3$ as a viscous colorless oil $(3.50 \mathrm{~g}, 14.3 \mathrm{mmol}, 73 \%)$.

$[\alpha]_{\mathrm{D}}{ }^{25}=-10.5(\mathrm{c}=1.0$ in $\mathrm{MeOH}) ; \mathbf{R}_{\boldsymbol{f}}=0.50\left(\mathrm{Et}_{2} \mathrm{O}: n\right.$-pentane $\left.1: 1\right) ;{ }^{1} \mathrm{H}-\mathrm{NMR}\left(400 \mathrm{MHz}, \mathrm{CDCl}_{3}\right)$ : $\delta=5.29\left(\mathrm{~d},{ }^{3} \mathrm{~J}_{\mathrm{HH}}=9.2 \mathrm{~Hz}, 1 \mathrm{H}, \mathrm{NH}\right), 4.44\left(\mathrm{dd},{ }^{3} \mathrm{~J}_{\mathrm{HH}}=9.2,5.9 \mathrm{~Hz}, 1 \mathrm{H}, \mathrm{H} 1\right), 3.07(\mathrm{~s}, 3 \mathrm{H}, \mathrm{H} 5 / 6)$, $2.94(\mathrm{~s}, 3 \mathrm{H}, \mathrm{H} 5 / 6), 1.97-1.84(\mathrm{~m}, 1 \mathrm{H}, \mathrm{H} 2), 1.40(\mathrm{~s}, 9 \mathrm{H}, \mathrm{H} 9), 0.93\left(\mathrm{~d},{ }^{3} \mathrm{~J}_{\mathrm{HH}}=6.8 \mathrm{~Hz}, 3 \mathrm{H}, \mathrm{H} 3 / 3^{\prime}\right)$, $0.87\left(\mathrm{~d},{ }^{3} \mathrm{JHH}_{\mathrm{HH}}=6.8 \mathrm{~Hz}, 3 \mathrm{H}, \mathrm{H} 3 / 3^{\prime}\right) \mathrm{ppm} ;{ }^{13} \mathrm{C}\left\{{ }^{1} \mathrm{H}\right\}-\mathrm{NMR}\left(101 \mathrm{MHz}, \mathrm{CDCl}_{3}\right): \delta=172.3(\mathrm{C} 4), 156.0$ (C7), 79.5 (C8), 55.0 (C1), 37.5 (C2), 35.7 (C5/6), 31.6 (C5/6), 28.5 (C9), 19.6 (C3/3'), 17.4 (C3/3') ppm; IR (ATR): $\tilde{v}=3304(\mathrm{w}), 2967$ (w), 2933 (w), $1704(\mathrm{~m}), 1638(\mathrm{~s}), 1490(\mathrm{~m}), 1415$ (w), $1392(\mathrm{~m}), 1365(\mathrm{~m}), 1311(\mathrm{w}), 1245(\mathrm{~m}), 1167(\mathrm{~s}), 1086(\mathrm{w}), 1042(\mathrm{w}), 1015(\mathrm{~m}), 979(\mathrm{w})$, 933 (w), 880 (w), 845 (w), 779 (w), 745 (w), 698 (w) cm ${ }^{-1}$; HR-ESI-MS: m/z: [M+Na] ${ }^{+}$calcd. for 
$\mathrm{C}_{12} \mathrm{H}_{24} \mathrm{~N}_{2} \mathrm{O}_{3} \mathrm{Na}^{+}:$267.1679; found: 267.1697 . The analytical data are in agreement with the literature. ${ }^{[16]}$

\section{(S)-2-Amino-N,N,3-trimethylbutanamide hydrochloric acid (S4)}

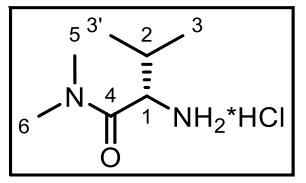

S3 (2.44 g, $10 \mathrm{mmol}, 1$ eq.) was dissolved in $\mathrm{MeOH}(100 \mathrm{~mL})$ and cooled to $0{ }^{\circ} \mathrm{C}$ before $\mathrm{AcCl}(4.2 \mathrm{~mL}, 60 \mathrm{mmol}, 6$ eq.) was added slowly. The reaction mixture was stirred for $6 \mathrm{~h}$ while warming up to room temperature. Removing of the volatiles in vacuo yielded $\mathbf{S} 4$ as a colorless hygroscopic solid (1.61 g, $8.90 \mathrm{mmol}, 89 \%)$.

$[\alpha]_{\mathrm{D}}{ }^{25}=+93.6(\mathrm{c}=1.0 \mathrm{in} \mathrm{MeOH}) ;{ }^{1} \mathrm{H}-\mathrm{NMR}\left(400 \mathrm{MHz},\left(\mathrm{CD}_{3}\right)_{2} \mathrm{SO}\right): \delta=8.21\left(\mathrm{~s}, 2 \mathrm{H}, \mathrm{NH}_{2}\right), 4.21-$ $4.13(\mathrm{~m}, 1 \mathrm{H}, \mathrm{H} 1), 3.04(\mathrm{~s}, 3 \mathrm{H}, \mathrm{H} 5), 2.89(\mathrm{~s}, 3 \mathrm{H}, \mathrm{H} 6), 2.12-2.00(\mathrm{~m}, 1 \mathrm{H}, \mathrm{H} 2), 0.97\left(\mathrm{~d},{ }^{3} J_{\mathrm{HH}}=\right.$ $\left.6.9 \mathrm{~Hz}, 3 \mathrm{H}, \mathrm{H} 3 / 3^{\prime}\right), 0.91\left(\mathrm{~d},{ }^{3} \mathrm{JHH}_{\mathrm{HH}}=6.9 \mathrm{~Hz}, 3 \mathrm{H}, \mathrm{H} 3 / 3^{\prime}\right) \mathrm{ppm} ;{ }^{13} \mathrm{C}\left\{{ }^{1} \mathrm{H}\right\}-\mathrm{NMR}\left(101 \mathrm{MHz},\left(\mathrm{CD}_{3}\right)_{2} \mathrm{SO}\right)$ : $\delta=168.1$ (C4), 53.9 (C1), 37.0 (C5), 35.3 (C6), 29.3 (C2), 18.5 (C3/3'), 17.2 (C3/3') ppm; IR (ATR): $\tilde{v}=2934(\mathrm{~m}), 2851(\mathrm{~m}), 2660(\mathrm{w}), 2618(\mathrm{w}), 1985(\mathrm{w}), 1640(\mathrm{~s}), 1564(\mathrm{~s}), 1504$ (s), 1482 (s), $1394(\mathrm{~m}), 1366(\mathrm{~s}), 1279(\mathrm{w}), 1249(\mathrm{w}), 1193(\mathrm{w}), 1176(\mathrm{w}), 1148(\mathrm{~m}), 1118(\mathrm{~m}), 1099(\mathrm{w})$, $1063(\mathrm{~m}), 1022(\mathrm{~m}), 932(\mathrm{w}), 893(\mathrm{w}), 819(\mathrm{w}), 796(\mathrm{w}), 717(\mathrm{~m}), 698(\mathrm{w}) \mathrm{cm}^{-1}$; HR-ESI-MS: $\mathrm{m} / \mathrm{z}$ : $[\mathrm{M}-\mathrm{Cl}]^{+}$calcd. for $\mathrm{C}_{7} \mathrm{H}_{17} \mathrm{~N}_{2} \mathrm{O}^{+}: 145.1335$; found: 145.1364 ; m.p. $=27-29^{\circ} \mathrm{C}$. The analytical data are in agreement with the literature. ${ }^{[16]}$

\section{(S)-2-Isothiocyanato- $N, N, 3$-trimethylbutanamide (S5)}

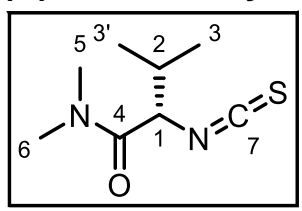

Following an adapted literature procedure, ${ }^{[17]} \mathbf{S 4}(1.08 \mathrm{~g}, 6 \mathrm{mmol}, 1 \mathrm{eq}$. was dissolved in $\mathrm{CH}_{2} \mathrm{Cl}_{2}(30 \mathrm{~mL})$ and a sat. aq. $\mathrm{NaHCO}_{3}(30 \mathrm{~mL})$ solution at $0^{\circ} \mathrm{C}$ and stirred for $5 \mathrm{~min}$ at $0^{\circ} \mathrm{C}$ before the stirring was stopped and $\mathrm{SCCl}_{2}(0.5 \mathrm{~mL}, 6.6 \mathrm{mmol}, 1.1 \mathrm{eq}$.) was added to the organic layer. After stirring for $2 \mathrm{~h}$ at $0{ }^{\circ} \mathrm{C}$, the organic layer was separated and the aqueous layer was extracted with $\mathrm{CH}_{2} \mathrm{Cl}_{2}(3 \times 20 \mathrm{~mL})$. The combined organic layers were dried over $\mathrm{Na}_{2} \mathrm{SO}_{4}$, filtered and the solvent and remaining thiophosgene was removed by distillation (do not remove the solvent outside a vented fume hood; thiophosgene is highly toxic). Purification by column chromatography $\left(\mathrm{SiO}_{2}, \mathrm{Et}_{2} \mathrm{O}: n\right.$-pentane $\left.3: 2\right)$ yielded $\mathbf{S} 5$ as a brown oil $(974 \mathrm{mg}$, $5.23 \mathrm{mmol}, 87 \%)$.

$[\alpha]_{\mathrm{D}}{ }^{25}=+61.6(\mathrm{c}=1.0$ in $\mathrm{MeOH}) ; \mathbf{R}_{f}=0.26\left(\mathrm{Et}_{2} \mathrm{O}\right.$ :n-pentane 3:2); ${ }^{1} \mathbf{H}-\mathrm{NMR}\left(500 \mathrm{MHz}, \mathrm{CDCl}_{3}\right)$ : $\delta=4.23\left(\mathrm{~d},{ }^{3} J_{\mathrm{HH}}=6.1 \mathrm{~Hz}, 1 \mathrm{H}, \mathrm{H} 1\right), 3.05(\mathrm{~s}, 3 \mathrm{H}, \mathrm{H} 5), 3.00(\mathrm{~s}, 3 \mathrm{H}, \mathrm{H} 6), 2.28-2.21(\mathrm{~m}, 1 \mathrm{H}, \mathrm{H} 2)$, $1.07\left(\mathrm{~d},{ }^{3} \mathrm{JHH}_{\mathrm{HH}}=6.7 \mathrm{~Hz}, 3 \mathrm{H}, \mathrm{H} 3 / 3^{\prime}\right), 1.02\left(\mathrm{~d},{ }^{3} \mathrm{JHH}_{\mathrm{HH}}=6.7 \mathrm{~Hz}, 3 \mathrm{H}, \mathrm{H} 3 / 3^{\prime}\right) \mathrm{ppm} ;{ }^{13} \mathrm{C}\left\{{ }^{1} \mathrm{H}\right\}-\mathrm{NMR}$ (126 MHz, $\mathrm{CDCl}_{3}$ ): $\delta$ = 166.9 (C4), 136.7 (C7), 63.7 (C1), 37.4 (C5), 36.5 (C6), 32.2 (C2), 20.0 (C3/3'), 18.0 (C3/3') ppm; IR (ATR): $\tilde{v}=2967$ (w), 2933 (w), 2875 (w), 2043 (s), 1652 (s), 1496 $(\mathrm{w}), 1464(\mathrm{w}), 1400(\mathrm{~m}), 1371(\mathrm{w}), 1351(\mathrm{~m}), 1319(\mathrm{w}), 1261(\mathrm{w}), 1166(\mathrm{w}), 1122(\mathrm{~m}), 1100(\mathrm{w})$, $1057(w), 976(w), 928(w), 853(\mathrm{~m}), 828(w), 742(\mathrm{~m}), 689(\mathrm{w}) \mathrm{cm}^{-1}$; HR-ESI-MS: $\mathrm{m} / \mathrm{z}:[\mathrm{M}+\mathrm{Na}]^{+}$ calcd. for $\mathrm{C}_{8} \mathrm{H}_{14} \mathrm{~N}_{2} \mathrm{OSNa} \mathrm{Na}^{+}$: 209.0719; found: 209.0731 .

\section{(1R,2R)-2-(2,5-Dimethyl-1H-pyrrol-1-yl)cyclohexan-1-amine (S6)}

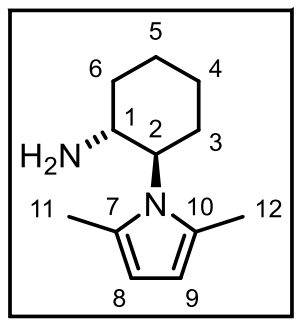

Following an adapted literature procedure, ${ }^{[18]}(R, R)$-diaminocyclohexane (1.14 g, $10 \mathrm{mmol}, 1$ eq.), 2,5-hexanedione (1.17 mL, $10 \mathrm{mmol}, 1$ eq.) and acetic acid $(0.57 \mathrm{~mL}, 10 \mathrm{mmol}, 1$ eq.) were dissolved in $\mathrm{MeOH}(50 \mathrm{~mL})$ and stirred at $50^{\circ} \mathrm{C}$ for $1 \mathrm{~h}$. The volatiles were removed under reduced pressure and the residue was redissolved in $\mathrm{CH}_{2} \mathrm{Cl}_{2}(50 \mathrm{~mL})$ and aq. $\mathrm{NaOH}$ solution $\left(4 \mathrm{M}, 50 \mathrm{~mL}\right.$ ). The separated aqueous layer was extracted with $\mathrm{CH}_{2} \mathrm{Cl}_{2}$ $(3 \times 30 \mathrm{~mL})$ and the combined organic layers were dried over $\mathrm{Na}_{2} \mathrm{SO}_{4}$, filtered and concentrated in vacuo. Purification by column chromatography $\left(\mathrm{SiO}_{2}, 1^{\text {st. }}\right.$ acetone: $\mathrm{CH}_{2} \mathrm{Cl}_{2}$ 10:90 - 30:70; $\left.2^{\text {nd }}: \mathrm{MeOH}: \mathrm{CH}_{2} \mathrm{Cl}_{2} 1: 99-3: 97\right)$ yielded $\mathbf{S} 6$ as an orange oil (1.18 g, $6.13 \mathrm{mmol}, 61 \%)$.

$[\alpha]_{\mathrm{D}}{ }^{25}=-48.8\left(\mathrm{c}=1.0\right.$ in $\left.\mathrm{CHCl}_{3}\right) ; \mathbf{R}_{\boldsymbol{f}}=0.10$ (TFA:MeOH: $\mathrm{CH}_{2} \mathrm{Cl}_{2}$ 1:4:95); ${ }^{1} \mathrm{H}-\mathrm{NMR}(500 \mathrm{MHz}$, $\left.\mathrm{CDCl}_{3}\right): \delta=5.78(\mathrm{~s}, 1 \mathrm{H}, \mathrm{H} 8 / 9), 5.75(\mathrm{~s}, 1 \mathrm{H}, \mathrm{H} 8 / 9), 3.60$ (td, $\left.{ }^{3} \mathrm{~J}_{\mathrm{HH}}=10.8,4.7 \mathrm{~Hz}, 1 \mathrm{H}, \mathrm{H} 2\right), 3.26$ $\left(\mathrm{td},{ }^{3} \mathrm{~J}_{\mathrm{HH}}=10.6,4.0 \mathrm{~Hz}, 1 \mathrm{H}, \mathrm{H} 1\right), 2.36(\mathrm{~s}, 3 \mathrm{H} ; \mathrm{H} 11 / 12), 2.23(\mathrm{~s}, 3 \mathrm{H}, \mathrm{H} 11 / 12), 2.10-2.01(\mathrm{~m}, 1 \mathrm{H}$, H6), $1.92-1.83\left(\mathrm{~m}, 3 \mathrm{H}, \mathrm{H} 3, \mathrm{H} 3\right.$ ', H4), $1.83-1.76(\mathrm{~m}, 1 \mathrm{H}, \mathrm{H} 5), 1.41-1.33\left(\mathrm{~m}, 2 \mathrm{H}, \mathrm{H} 4{ }^{\prime}, \mathrm{H} 5\right.$ '), $1.28-1.15\left(\mathrm{~m}, 1 \mathrm{H}, \mathrm{H} 6{ }^{\prime}\right) \mathrm{ppm} ;{ }^{13} \mathrm{C}\left\{{ }^{1} \mathrm{H}\right\}-N M R\left(126 \mathrm{MHz}, \mathrm{CDCl}_{3}\right): \delta=130.0(\mathrm{C} 7 / 10), 127.0$ 
(C7/10), 108.0 (C8/9), 105.4 (C8/9), 63.9 (C2), 53.1 (C1), 35.6 (C6), 31.5 (C3), 26.4 (C4), 25.3 (C5), 15.4 (C11/12), 13.9 (C11/12) ppm; IR (ATR): $\tilde{v}=2934$ (w), $2859(w), 1659(w), 1553(w)$, $1518(w), 1448(w), 1395(m), 1291(m), 1215(w), 1143(w), 1024(w), 979(w), 952(w), 870$ (w), 850 (w), 745 (s), 664 (m) cm ${ }^{-1}$; HR-ESI-MS: $m / z$ : $[\mathrm{M}+\mathrm{H}]^{+}$calcd. for $\mathrm{C}_{12} \mathrm{H}_{20} \mathrm{~N}_{2} \mathrm{H}^{+}:$193.1699; found: 193.1701 . The analytical data are in agreement with the literature. ${ }^{[18]}$

(S)-2-(3-((1R,2R)-2-(2,5-Dimethyl-1H-pyrrol-1-yl)cyclohexyl)thioureido)-N,N,3trimethylbutanamide $(\mathrm{A})$

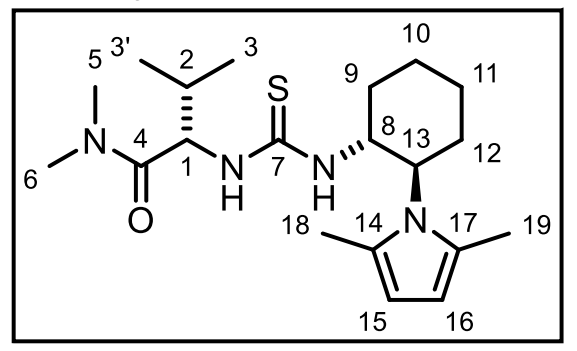

Following an adapted literature procedure ${ }^{[18]}$ S5 $(745 \mathrm{mg}$, $4 \mathrm{mmol}, 1.0$ eq.) and $\mathbf{S 6}$ (846 mg, $4.4 \mathrm{mmol}, 1.1$ eq.) were dissolved in $\mathrm{CH}_{2} \mathrm{Cl}_{2} \quad(35 \mathrm{~mL})$ and stirred at room temperature for $22 \mathrm{~h}$. The solvent was removed and purification by column chromatography (1 $1^{\text {st: }} \mathrm{MeOH}: \mathrm{CH}_{2} \mathrm{Cl}_{2}$ $0: 1-1.5: 98.5 ; 2^{\text {nd }}:$ EtOAc:cyclohexane $\left.3: 7-1: 1\right)$ yielded $\mathbf{A}$ (1.01 g, $2.68 \mathrm{mmol}, 67 \%)$ as an orange solid.

$[\alpha]_{\mathrm{D}}{ }^{25}=+44.8\left(\mathrm{c}=0.5\right.$ in $\left.\mathrm{CHCl}_{3}\right) ; \mathbf{R}_{\boldsymbol{f}}=0.14$ (EtOAc:cyclohexane 2:3); ${ }^{1} \mathbf{H}-\mathbf{N M R}(500 \mathrm{MHz}$, $\left.\mathrm{CDCl}_{3}\right): \delta=6.57\left(\mathrm{br} \mathrm{s}, 1 \mathrm{H}, \mathrm{NH}^{7-8}\right), 6.21\left(\mathrm{br} \mathrm{s}, 1 \mathrm{H}, \mathrm{NH}^{1-7}\right), 5.70(\mathrm{~s}, 2 \mathrm{H}, \mathrm{H} 15, \mathrm{H} 16), 5.27$ (br s, $1 \mathrm{H}$, $\mathrm{H} 13), 4.38$ (br s, 1H, H8), $3.83-3.73(\mathrm{~m}, 1 \mathrm{H}, \mathrm{H} 1), 3.14(\mathrm{~s}, 3 \mathrm{H}, \mathrm{H} 5), 2.94(\mathrm{~s}, 3 \mathrm{H}, \mathrm{H} 6), 2.57-$ 2.10 (m, 7H, H9, H18, H19), $2.10-1.68$ (m, 5H, H2, H10, H11, H12, H12'), $1.45-1.13$ (m, 3H, $\mathrm{H} 9$ ', $\left.\mathrm{H} 10, \mathrm{H} 11^{\prime}\right), 0.87\left(\mathrm{~d},{ }^{3} \mathrm{JHH}_{\mathrm{HH}}=6.8 \mathrm{~Hz}, 6 \mathrm{H}, \mathrm{H} 3\right) \mathrm{ppm} ;{ }^{13} \mathrm{C}\left\{{ }^{1} \mathrm{H}\right\}-\mathrm{NMR}\left(151 \mathrm{MHz}, \mathrm{CDCl}_{3}\right): \delta=182.1$ (C7), 172.8 (C4), 129.1 (C14), 127.3 (C17), 108.7 (C15/16), 106.1 (C15/16), 59.9 (C1), 59.6 (C13), 56.1 (C8), 38.0 (C5), 35.8 (C6), 34.2 (C9), 32.3 (C2), 32.1 (C12), 26.0 (C10/11), 24.9 (C10/11), 19.1 (C3/3'), 18.8 (C3/3'), 15.6 (C18/19), 13.7 (C18/19) ppm; IR (ATR): $\tilde{v}=3247$ (w), 3048 (w), 2930 (w), 2859 (w), 2053 (w), $1632(\mathrm{~s}), 1546(\mathrm{~s}), 1520(\mathrm{~m}), 1465(\mathrm{w}), 1447(\mathrm{w}), 1418$ (w), 1396 (s), $1316(w), 1291(w), 1260(w), 1230(w), 1180(w), 1130(w), 1093(w), 1069(w)$, 1012 (w), 978 (w), 954 (w), 871 (w), 843 (w), 801 (w), 739 (w) $\mathrm{cm}^{-1}$; HR-ESI-MS: m/z: [M+Na] $]^{+}$ calcd. for $\mathrm{C}_{20} \mathrm{H}_{34} \mathrm{~N}_{4} \mathrm{OSNa}{ }^{+}$: 401.2346; found: 401.2347 ; m.p.: $=141-143^{\circ} \mathrm{C}$. The analytical data are in agreement with the literature. ${ }^{[18]}$

\section{(S)-2-(3-((1R,2R)-2-(2,5-Dimethyl-1H-pyrrol-1-yl)cyclohexyl)thioureido)- N,N,3-}

trimethylbutanamide $(B)$

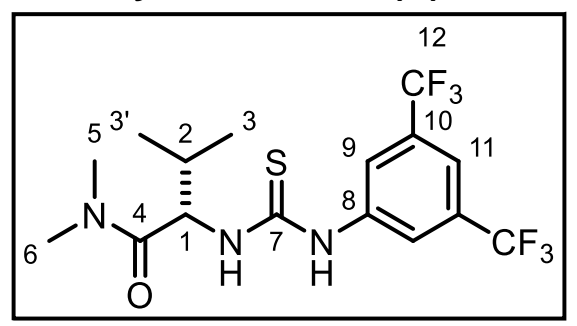

Following an adapted literature procedure, ${ }^{[18]} \mathbf{S 4}$ was converted to the free amine by dissolving in $\mathrm{CH}_{2} \mathrm{Cl}_{2}$ and washing with a sat. aq. $\mathrm{NaHCO}_{3}$ solution, drying over $\mathrm{Na}_{2} \mathrm{SO}_{4}$, filtering and concentration in vacuo. The free amine of $\mathbf{S 4}(115 \mathrm{mg}, 0.8 \mathrm{mmol}, 1 \mathrm{eq}$.) was dissolved in $\mathrm{CH}_{2} \mathrm{Cl}_{2}(5 \mathrm{~mL})$ and $\mathrm{NEt}_{3}(0.11 \mathrm{~mL}, 0.8 \mathrm{mmol}$, 1 eq.) was slowly added. After stirring for $5 \mathrm{~min}$ at room temperature, 3,5-bis(trifluoromethyl)phenyl isothiocyanate $(0.15 \mathrm{~mL}$, $0.8 \mathrm{mmol}, 1 \mathrm{eq}$.) was added and the reaction mixture was stirred at room temperature over night. The solvent was removed in vacuo and purification by column chromatography $\left(\mathrm{SiO}_{2}\right.$, EtOAc:cyclohexane 2:3) yielded $\mathbf{B}$ as an off-white solid (215 mg, $0.52 \mathrm{mmol}, 65 \%$ ).

$[\alpha]_{D}{ }^{25}=-93.8\left(\mathrm{C}=0.5\right.$ in $\left.\mathrm{CHCl}_{3}\right) ; \mathbf{R}_{\boldsymbol{f}}=0.13$ (EtOAc:cyclohexane 2:3); ${ }^{1} \mathrm{H}-\mathrm{NMR}$ (600 MHz, $\left.\mathrm{CDCl}_{3}\right): \delta=9.33\left(\mathrm{~s}, 1 \mathrm{H}, \mathrm{NH}^{7-8}\right), 8.37\left(\mathrm{~d},{ }^{3} \mathrm{~J}_{\mathrm{HH}}=7.7 \mathrm{~Hz}, 1 \mathrm{H}, \mathrm{NH}^{1-7}\right), 8.0(\mathrm{~s}, 2 \mathrm{H}, \mathrm{H} 9), 7.48(\mathrm{~s}, 1 \mathrm{H}$, $\mathrm{H} 11), 5.25\left(\mathrm{t},{ }^{3} \mathrm{JHH}_{\mathrm{H}}=8.0 \mathrm{~Hz}, 1 \mathrm{H}, \mathrm{H} 1\right), 3.39(\mathrm{~s}, 3 \mathrm{H}, \mathrm{H} 5 / 6), 3.05(\mathrm{~s}, 3 \mathrm{H}, \mathrm{H} 5 / 6), 2.08(\mathrm{~m}, 1 \mathrm{H}, \mathrm{H} 2)$, $1.13\left(\mathrm{~d},{ }^{3} \mathrm{~J}_{\mathrm{HH}}=6.8 \mathrm{~Hz}, 3 \mathrm{H}, \mathrm{H} 3 / 3^{\prime}\right), 1.09\left(\mathrm{~d},{ }^{3} \mathrm{JHH}_{\mathrm{HH}}=6.8 \mathrm{~Hz}, 3 \mathrm{H}, \mathrm{H} 3 / 3^{\prime}\right) \mathrm{ppm} ;{ }^{13} \mathrm{C}\left\{{ }^{1} \mathrm{H}\right\}-\mathrm{NMR}(151$ $\left.\mathrm{MHz}, \mathrm{CDCl}_{3}\right): \delta=181.6(\mathrm{C} 7), 174.9(\mathrm{C} 4), 140.7(\mathrm{C} 8), 131.4\left(\mathrm{q},{ }^{2} J_{\mathrm{CF}}=33.4 \mathrm{~Hz}, \mathrm{C} 10\right), 123.3(\mathrm{q}$, $\left.{ }^{1} J_{\mathrm{CF}}=272.8 \mathrm{~Hz}, \mathrm{C} 12\right), 122.7\left(\mathrm{q},{ }^{3} J_{\mathrm{CF}}=3.8 \mathrm{~Hz}, \mathrm{C} 9\right), 117.5\left(\mathrm{q},{ }^{3} \mathrm{~J}_{\mathrm{CF}}=3.9 \mathrm{~Hz}, \mathrm{C} 11\right), 59.7(\mathrm{C} 1)$, 38.5 (C5/6), 36.4 (C5/6), 31.9 (C2), 19.4 (C3/3'), 19.2 (C3/3') ppm; ${ }^{19} \mathrm{~F}-\mathrm{NMR}$ (564 MHz): $\delta=-63.13$ ppm; IR (ATR): $\tilde{v}=3315(w), 3268(w), 3099(w), 3975(w), 1645(w), 1613(w), 1597$ $(\mathrm{w}), 1541(\mathrm{~m}), 1474(\mathrm{w}), 1422(\mathrm{w}), 1407(\mathrm{w}), 1385(\mathrm{~m}), 1271(\mathrm{~s}), 1219(\mathrm{w}), 1170(\mathrm{~s}), 1120(\mathrm{~s})$, $1108(\mathrm{~s}), 1000(\mathrm{w}), 966(\mathrm{~m}), 929(\mathrm{w}), 907(\mathrm{w}), 879(\mathrm{~m}), 849(\mathrm{w}), 836(\mathrm{w}), 771(\mathrm{w}), 721(\mathrm{~m}), 697$ (w), $677(\mathrm{~m}) \mathrm{cm}^{-1}$; HR-ESI-MS: $\mathrm{m} / \mathrm{z}$ : [M+Na] ${ }^{+}$calcd. for $\mathrm{C}_{16} \mathrm{H}_{19} \mathrm{~N}_{3} \mathrm{OSF}_{6} \mathrm{Na}^{+}$: 438.1045; found: 438.1046; m.p.: $=142-144{ }^{\circ} \mathrm{C}$. The analytical data are in agreement with the literature. ${ }^{[18]}$ 


\section{Reaction optimization}

\section{Optimization and upscaling of the isomerization}

Isomerization reactions were performed similar to general procedure $\mathbf{C}$ with differences shown in Table 19.

Table 19: Optimization of the isomerization of $\boldsymbol{E}-1$ to $\mathbf{Z}-1$.

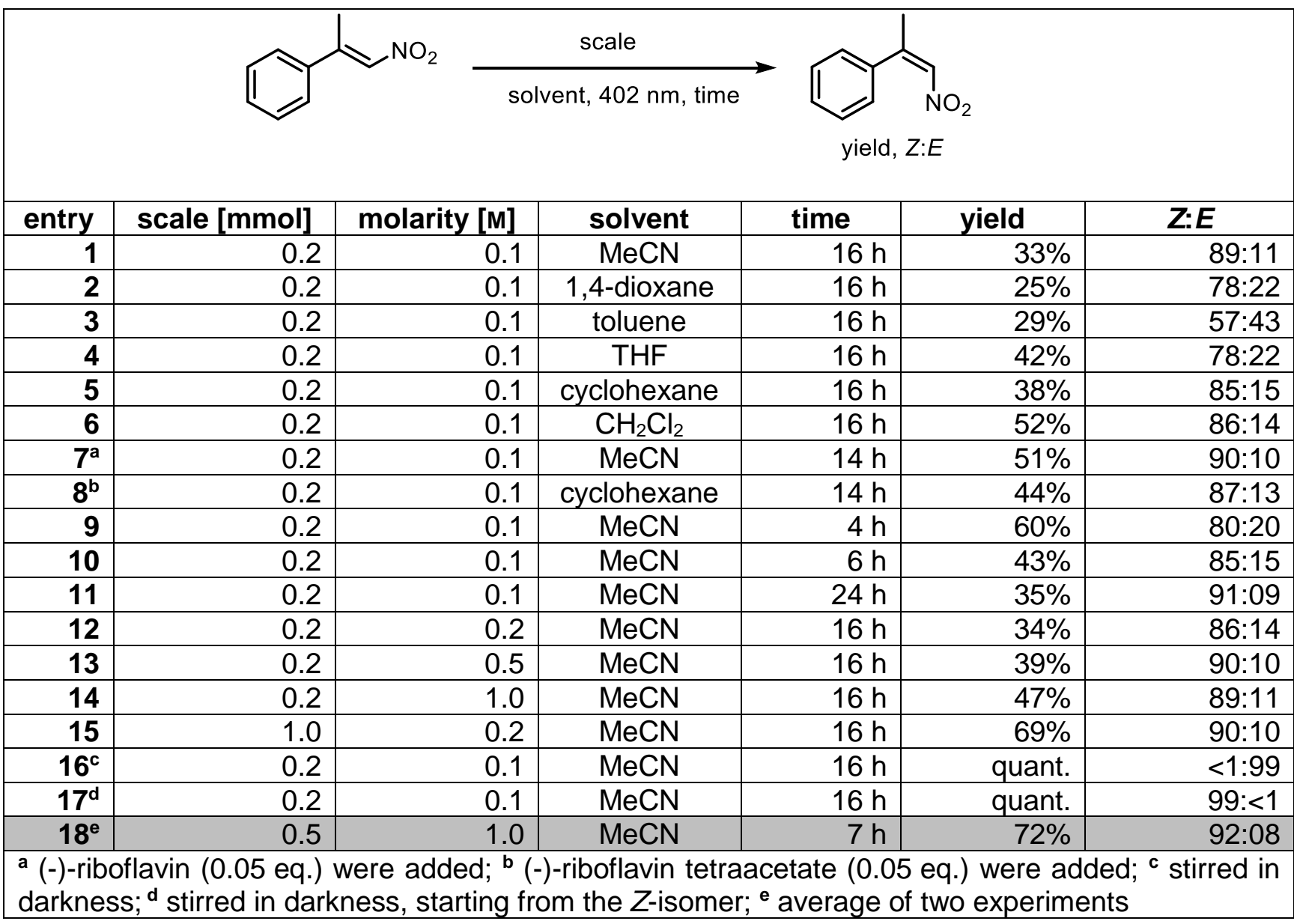

\section{Upscaling}

Isomerization reaction was performed in a $25 \mathrm{~mL}$ round bottom flask according to general procedure $\mathbf{C}$ using a small LED ( $402 \mathrm{~nm})$. The reaction was run on a $2.5 \mathrm{mmol}$ scale under irradiation for $18 \mathrm{~h}$.<smiles>C/C(=C\[N+](=O)[O-])c1cccc(Cl)c1</smiles><smiles>C/C(=C/[N+](=O)[O-])c1cccc(Cl)c1</smiles>

$82 \%$ yield

Scheme 2: Upscaling of the isomerization of $E-9$ to $Z-9$. 


\section{Optimization of the hydrogenation using catalyst $\mathrm{A}$}

Hydrogenations were performed similar to general procedure $\mathbf{E}$ with differences shown in Table 20.

Table 20: Optimization of hydrogenation of $E-1$ or $Z-1$ to $(S)-13$ or $(R)-13$.

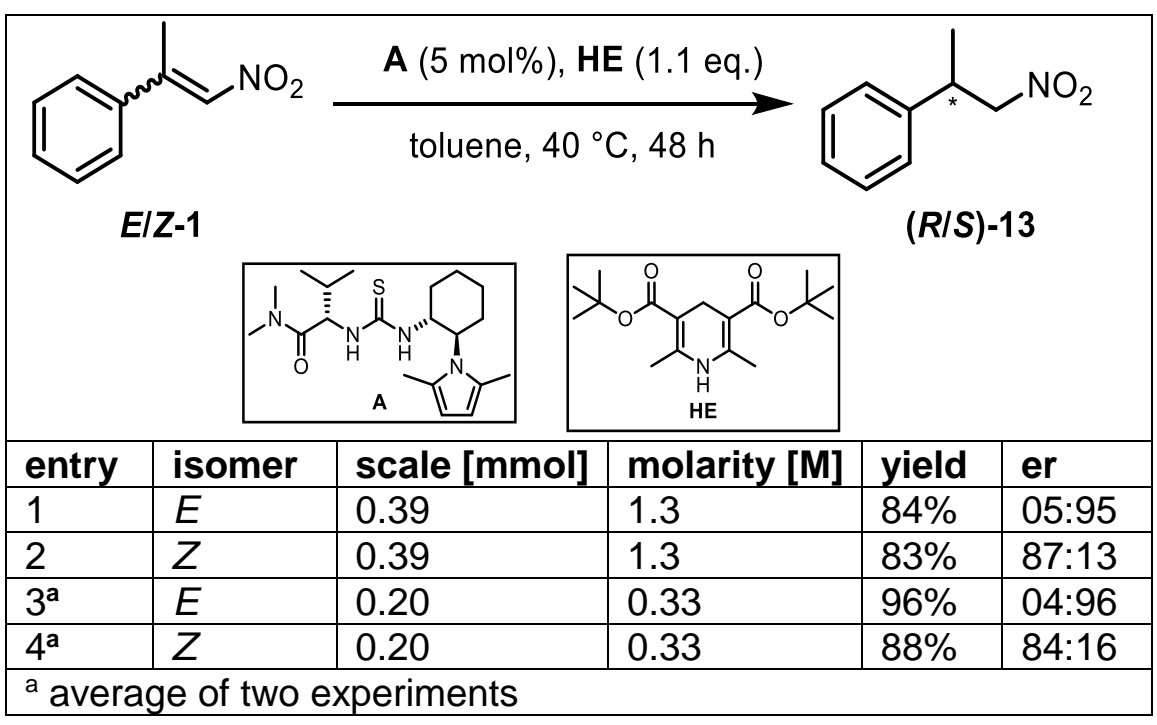




\section{Optimization of the hydrogenation using the Z-isomer}

Hydrogenations were performed similar to general procedure $\mathbf{F}$ with differences shown in Table 21.

Table 21: Optimization of the hydrogenation of $\boldsymbol{Z}-\mathbf{1}$ to $(\boldsymbol{R}) \mathbf{- 1 3}$ and control experiments.

\begin{tabular}{|c|c|c|c|c|c|}
\hline & & $\mathrm{NO}_{\mathrm{Z}-1}$ & $\frac{(x \mathrm{~mol} \%), \mathrm{HE}(\mathrm{x}}{\text { uene, } 40^{\circ} \mathrm{C}, 48}$ & $\overbrace{N}^{\prime \prime}$ & \\
\hline entry & cat. & cat. loading & HE loading & yield & $e^{a}$ \\
\hline $1^{b}$ & $A$ & $5.0 \%$ & $1.1 \mathrm{eq}$. & $88 \%$ & $84: 16$ \\
\hline 2 & A & $10.0 \%$ & $1.1 \mathrm{eq}$. & $61 \%$ & $65: 35$ \\
\hline 3 & $A$ & $20.0 \%$ & $1.1 \mathrm{eq}$. & $86 \%$ & $55: 45$ \\
\hline 4 & $A$ & $1.0 \%$ & $1.1 \mathrm{eq}$. & $71 \%$ & $61: 39$ \\
\hline 5 & $A$ & $2.5 \%$ & 1.1 eq. & $58 \%$ & $76: 24$ \\
\hline 6 & A & $5.0 \%$ & 3.0 eq. & $73 \%$ & $78: 22$ \\
\hline 7 & $A$ & $5.0 \%$ & 4.4 eq. & $75 \%$ & $77: 23$ \\
\hline 8 & $A$ & $5.0 \%$ & 5.0 eq. & $38 \%$ & $75: 25$ \\
\hline $9^{b}$ & B & $5.0 \%$ & $1.1 \mathrm{eq}$. & $94 \%$ & $93: 07$ \\
\hline $10^{b, c}$ & B & $5.0 \%$ & $1.1 \mathrm{eq}$. & $86 \%$ & 09:91 \\
\hline 11 & - & - & $1.1 \mathrm{eq}$. & $89 \%{ }^{d}$ & $50: 50$ \\
\hline $12^{c}$ & - & - & $1.1 \mathrm{eq}$. & $95 \%{ }^{\mathrm{e}}$ & $50: 50$ \\
\hline 13 & $A$ & $5.0 \%$ & - & - & - \\
\hline 14 & B & $5.0 \%$ & - & - & - \\
\hline $15^{c}$ & $A$ & $5.0 \%$ & - & - & - \\
\hline $16^{c}$ & $B$ & $5.0 \%$ & - & - & - \\
\hline $\begin{array}{l}\text { a where } \\
\text { (alkane:2 } \\
90:<1: 8: 2\end{array}$ & $\begin{array}{l}\text { able } \\
\text { giois }\end{array}$ & $\begin{array}{l}\text { iverage of two } \\
\text { er } \quad 40: 11: 35 \text { : }\end{array}$ & $\begin{array}{l}\text { experiments; } \\
\text { ); } \quad \text { combine }\end{array}$ & $\begin{array}{l}\text { g from } E-1 \\
\text { eld } \quad \text { (alkar }\end{array}$ & $\begin{array}{l}\text { nbined } \\
\text { :regiois }\end{array}$ \\
\hline
\end{tabular}




\section{Analysis of Regioisomer Hydrogenation}

Throughout the isomerization scope small quantities of regioisomer could not be separated from the corresponding Z-isomer for a small number of substrates. However, during hydrogenation reactions the regioisomer was retained in similar quantites. Despite this, we looked to analyse the hydrogenation of the regioisomer to disclude any negative or cooperative effects it may have. Hydrogenations were performed similar to general procedure $\mathbf{F}$ with differences shown in Scheme 3.<smiles>C=C(C[N+](=O)[O-])c1ccccc1</smiles>

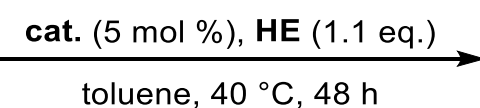<smiles>C/C(=C\[N+](=O)[O-])c1ccccc1</smiles>

\begin{tabular}{c|c|c} 
cat. & yield & $(\boldsymbol{R}: S)$ \\
\hline A & $77 \%$ & $29: 71$ \\
B & $49 \%$ & $57: 43$
\end{tabular}

Scheme 3: Hydrogenation of the regioisomer.

As observed hydrogenation did occur in much smaller quantities in comparison to $E$ - and $Z$-isomers. This is presumably due to a preliminary, in situ, positional isomerization to reform the conjugated system followed by a subsequent hydrogenation. The data supports this hypothesis as enantioselectivity is scrambled demonstrating a mixture of $E$ - and Z-isomer are formed and subsequently hydrogenated. Notably catalyst $\mathbf{A}$ prefers formation of the enantiomer derived from the $E$-isomer. As hydrogenation of the Z-isomer (in which small quantities of regioisomer persist) proceeded smoothly, this indicates that the presence of regioisomer has no effect on reaction output.

\section{One Pot Isomerization-Hydrogenation of $E-1$ to $(R)-13$}

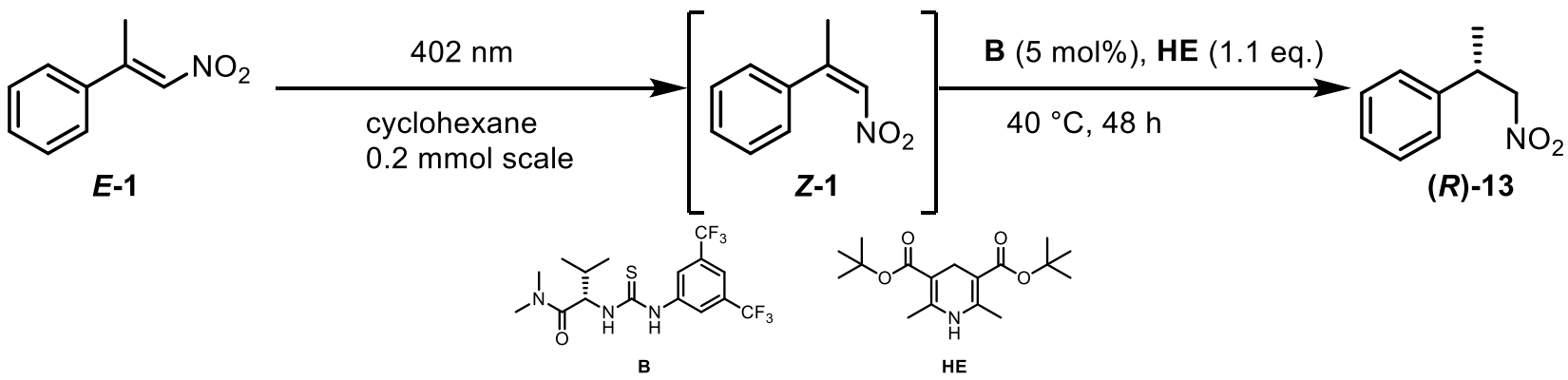

The one pot isomerization-hydrogenation was conducted as follows:

In a flame dried $2 \mathrm{~mL}$ vial, the $(E)$-nitrostyrene $(0.2 \mathrm{mmol}$, 1 eq.) was dissolved in dry cyclohexane $(616 \mu \mathrm{L}, 0.32 \mathrm{M})$. The vial was sealed with a rubber septum and parafilm and the mixture was degassed by bubbling argon through it with a needle for ca. $2 \mathrm{~min}$. The vial was connected to a balloon filled with argon and placed over the LED in the shown irradiation set up (Figure 2). After irradiation at $402 \mathrm{~nm}$ for $7 \mathrm{~h}$, catalyst B $(4.2 \mathrm{mg}, 0.01 \mathrm{mmol}, 0.05 \mathrm{eq}$.) and the Hantzsch ester (di-tert-butyl 2,6-dimethyl-1,4-dihydropyridine-3,5-dicarboxylate) (68 mg, $0.22 \mathrm{mmol}, 1.1$ eq.) were added. A rubber septum and parafilm were used to seal the vial, which was then placed in a preheated metal heating block at $40^{\circ} \mathrm{C}$ for $48 \mathrm{~h}$ (Figure 3). After that time, the crude reaction mixture was filtered through a plug of silica, which was rinsed with $\mathrm{Et}_{2} \mathrm{O}: n-$ pentane (2:98) to remove the catalyst and most of the oxidized Hantzsch ester. The filtrate was concentrated in vacuo and purified by column chromatography to yield the $(R)-13$ as a colorless oil $(26.7 \mathrm{mg}, 0.16 \mathrm{mmol}, 81 \%)$. The enantiomeric ratio was found to be $83: 17$, determined via chiral HPLC (Reprosil OM, $n$-hexane:iPrOH 97:3, $1.0 \mathrm{~mL} / \mathrm{min}, \lambda=210 \mathrm{~nm}$ ). 


\section{Synthesis of drug scaffolds}

\section{General procedure $\mathrm{G}$ for the synthesis of sulfonamides}<smiles>C/C(=C\[N+](=O)[O-])c1ccc(Cl)cc1</smiles>

$E / Z-5$

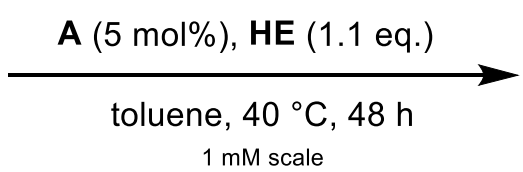

$1 \mathrm{mM}$ scale<smiles>CC(C[N+](=O)[O-])c1ccc(Cl)cc1</smiles>

(R/S)-17

from $E: 87 \%$, er $05: 95$

from Z: $87 \%$, er $91: 09$

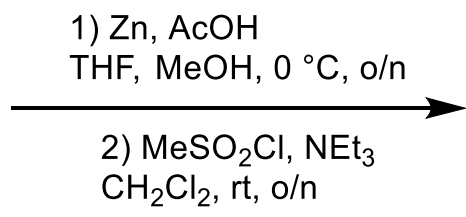

$\mathrm{CH}_{2} \mathrm{Cl}_{2}, \mathrm{rt}, \mathrm{o} / \mathrm{n}$<smiles>CC(CNS(C)(=O)=O)c1ccc(Cl)cc1</smiles>

(R/S)-22

from $E 55 \%$, er 08:92 from $Z 61 \%$, er $84: 16$

Scheme 4: Preparation of chiral sulfonamides.

Following an adapted literature procedure ${ }^{[7]}(\boldsymbol{R} / \boldsymbol{S})-17$ (1.0 eq.) was dissolved in THF:MeOH $(10: 1,0.25 \mathrm{M})$ and cooled to $0{ }^{\circ} \mathrm{C}$ before $\mathrm{Zn}$ dust (5 eq.) and $\mathrm{AcOH}$ (30 eq.) were added. The ice bath was removed and the reaction mixture was stirred at room temperature for $24 \mathrm{~h}$. $\mathrm{Zn}$ was removed by filtration and the residue was diluted with $\mathrm{H}_{2} \mathrm{O}$. The $\mathrm{pH}$ of the filtrate was adjusted to $\mathrm{pH} 11$ with $\mathrm{NaOH}(5 \mathrm{M})$ and organics were extracted with EtOAc $(3 \mathrm{x})$. The combined organic layers were washed with brine, dried over $\mathrm{Na}_{2} \mathrm{SO}_{4}$, filtered and concentrated in vacuo to yield the crude amine which was used for the next step without further analysis and purification. The amine from the previous step (1.0 eq.) was dissolved in $\mathrm{CH}_{2} \mathrm{Cl}_{2}(0.25 \mathrm{M})$, cooled to $0{ }^{\circ} \mathrm{C}$ before $\mathrm{NEt}_{3}$ (5.74 eq.) and methanesulfonylchloride (1.50 eq.) were added dropwise. After one hour at $0{ }^{\circ} \mathrm{C}$ the reaction mixture was stirred at room temperature over night. Then the reaction mixture was diluted with $\mathrm{Et}_{2} \mathrm{O}$ and $\mathrm{H}_{2} \mathrm{O}$ was added. The separated organic layer was washed with $\mathrm{HCl}(0.2 \mathrm{M})$, sat. aq. $\mathrm{NaHCO}_{3}$ and brine before being dried over $\mathrm{MgSO}_{4}$. After filtration and concentration in vacuo, the crude residue was purified by column chromatography $\left(\mathrm{SiO}_{2}, \mathrm{EtOAc:cyclohexane)}\right.$ yielding the sulfonamide.

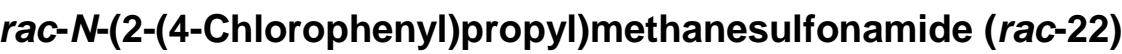

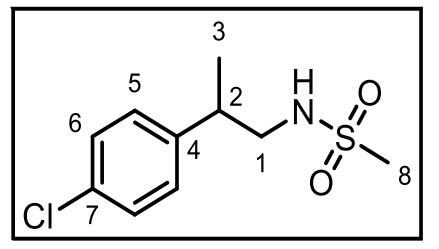

Prepared according to general procedure G, rac-17 $(250 \mathrm{mg}$, $1.25 \mathrm{mmol}, 1.0$ eq.) in THF:MeOH (10:1, $5 \mathrm{~mL})$ was reduced to the amine using $\mathrm{Zn}$ dust (409 mg, $6.25 \mathrm{mg}, 5$ eq.) and $\mathrm{AcOH}(2.14 \mathrm{~mL}$, $37.5 \mathrm{mmol}, 30$ eq.). The crude amine was treated with $\mathrm{NEt}_{3}(1.0 \mathrm{~mL}$, $7.17 \mathrm{mmol}, 5.74$ eq.) and methanesulfonyl chloride $(145 \mu \mathrm{L}$, $1.88 \mathrm{mmol}, 1.50$ eq.) in $\mathrm{CH}_{2} \mathrm{Cl}_{2}(5 \mathrm{~mL})$ to yield rac-22 as a colorless solid $(212.0 \mathrm{mg}, 0.86 \mathrm{mmol}, 68 \%)$ after purification by column chromatography $\left(\mathrm{SiO}_{2}, \mathrm{EtOAc}\right.$ : cyclohexane 15:85-30:70).

\section{(S)-N-(2-(4-Chlorophenyl)propyl)methanesulfonamide ((S)-22)}

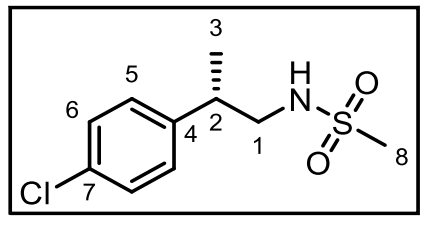

Prepared according to general procedure G, (S)-17 (er: 05:95, $50 \mathrm{mg}, 0.25 \mathrm{mmol}, 1.0$ eq.) in THF: $\mathrm{MeOH}(10: 1,5 \mathrm{~mL})$ was reduced to the amine using $\mathrm{Zn}$ dust (82 $\mathrm{mg}, 1.25 \mathrm{mg}, 5$ eq.) and $\mathrm{AcOH}$ $\left(0.43 \mathrm{~mL}, 7.5 \mathrm{mmol}, 30\right.$ eq.). The crude amine was treated with $\mathrm{NEt}_{3}$ (0.2 mL, $1.43 \mathrm{mmol}, 5.74$ eq.) and methanesulfonyl chloride $(29 \mu \mathrm{L}$, 
$0.38 \mathrm{mmol}, 1.50$ eq.) in $\mathrm{CH}_{2} \mathrm{Cl}_{2}(1 \mathrm{~mL})$ to yield $(S)-22$ as a colorless solid $(34.0 \mathrm{mg}, 0.14 \mathrm{mmol}$, $55 \%)$ after purification by column chromatography $\left(\mathrm{SiO}_{2}\right.$, EtOAc: cyclohexane 15:85 - 30:70). The enantiomeric ratio was found to be 08:92 by chiral HPLC (Reprosil Chiral-AM, $n$-hexane: $\mathrm{PrOH}$ 98:2, $1.0 \mathrm{~mL} / \mathrm{min}, \lambda=210 \mathrm{~nm}, \mathrm{t}_{\mathrm{R}}$ (major): $37.839 \mathrm{~min}, \mathrm{t}_{\mathrm{R}}$ (minor): $43.132 \mathrm{~min}$ ).

\section{(R)-N-(2-(4-Chlorophenyl)propyl)methanesulfonamide ((R)-22)}

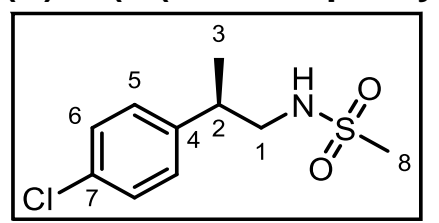

Prepared according to general procedure $\mathbf{G},(\boldsymbol{R}) \mathbf{- 1 7}$ (er: 91:09, $50 \mathrm{mg}, 0.25 \mathrm{mmol}, 1.0$ eq.) in THF:MeOH $(10: 1,5 \mathrm{~mL})$ was reduced to the amine using $\mathrm{Zn}$ dust ( $82 \mathrm{mg}, 1.25 \mathrm{mg}, 5$ eq.) and $\mathrm{AcOH}$ ( $0.43 \mathrm{~mL}, 7.5 \mathrm{mmol}, 30$ eq.). The crude amine was treated with $\mathrm{NEt}_{3}$ (0.2 mL, $1.43 \mathrm{mmol}, 5.74$ eq.) and methanesulfonyl chloride $(29 \mu \mathrm{L}$, $0.38 \mathrm{mmol}, 1.50$ eq. $)$ in $\mathrm{CH}_{2} \mathrm{Cl}_{2}(1 \mathrm{~mL})$ to yield $(\boldsymbol{R})-22$ as a colorless solid ( $38.0 \mathrm{mg}, 0.15 \mathrm{mmol}, 61 \%)$ after purification by column chromatography $\left(\mathrm{SiO}_{2}\right.$, EtOAc: cyclohexane 15:85 - 30:70). The enantiomeric ratio was found to be $84: 16$ by chiral HPLC Reprosil Chiral-AM, $n$-hexane: $/ \mathrm{PrOH}$ 98:2, $1.0 \mathrm{~mL} / \mathrm{min}, \lambda=210 \mathrm{~nm}, \mathrm{t}_{\mathrm{R}}$ (major): $37.161 \mathrm{~min}, \mathrm{t}_{\mathrm{R}}$ (minor): $42.785 \mathrm{~min}$ ).

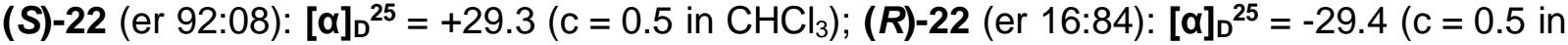
$\left.\mathrm{CHCl}_{3}\right) ; \mathbf{R}_{\boldsymbol{f}}=0.33$ (EtOAc:cyclohexane, 20:80); ${ }^{1} \mathrm{H}-\mathrm{NMR}\left(400 \mathrm{MHz}, \mathrm{CDCl}_{3}\right): \delta=7.34-7.29(\mathrm{~m}$, $2 \mathrm{H}, \mathrm{H} 6), 7.18-7.14(\mathrm{~m}, 2 \mathrm{H}, \mathrm{H} 5), 4.13-3.94(\mathrm{~m}, 1 \mathrm{H}, \mathrm{NH}), 3.38-3.30(\mathrm{~m}, 1 \mathrm{H}, \mathrm{H} 1), 3.25-3.15$ $\left(\mathrm{m}, 1 \mathrm{H}, \mathrm{H} 1^{\mathrm{c}}\right), 3.02-2.92(\mathrm{~m}, 1 \mathrm{H}, \mathrm{H} 2), 2.83(\mathrm{~s}, 3 \mathrm{H}, \mathrm{H} 8), 1.30\left(\mathrm{~d},{ }^{3} \mathrm{~J}_{\mathrm{HH}}=6.9 \mathrm{~Hz}, 3 \mathrm{H}, \mathrm{H} 3\right) \mathrm{ppm}$; ${ }^{13} \mathrm{C}\left\{{ }^{1} \mathrm{H}\right\}-N M R\left(101 \mathrm{MHz}, \mathrm{CDCl}_{3}\right): \delta=141.5$ (C4), 133.1 (C7), 129.2 (C6), 128.8 (C5), 50.0 (C1), 40.7 (C8), 40.1 (C2), 19.1 (C3) ppm; IR (ATR): v = 3244 (w), 2915 (w), 1491 (w), 1452 (w), 1436 $(w), 1411(w), 1384(w), 1345(w), 1335(w), 1311(s), 1270(w), 1196(w), 1155(m), 1125(\mathrm{~m})$, $1112(\mathrm{w}), 1090(\mathrm{~m}), 1070(\mathrm{~s}), 1023(\mathrm{~m}), 1011(\mathrm{~m}), 983(\mathrm{~m}), 925(\mathrm{w}), 894(\mathrm{w}), 847(\mathrm{~m}), 822(\mathrm{~s})$, $784(\mathrm{~m}), 760$ (s), 690 (w) cm ${ }^{-1}$; HR-ESI-MS: $\mathrm{m} / \mathrm{z}$ : $[\mathrm{M}+\mathrm{Na}]^{+}$calcd. for $\mathrm{C}_{10} \mathrm{H}_{14} \mathrm{NO}_{2} \mathrm{SCINa}^{+}$: 270.0326; found: 270.0320 ; m.p.: $=90-92^{\circ} \mathrm{C}$. 


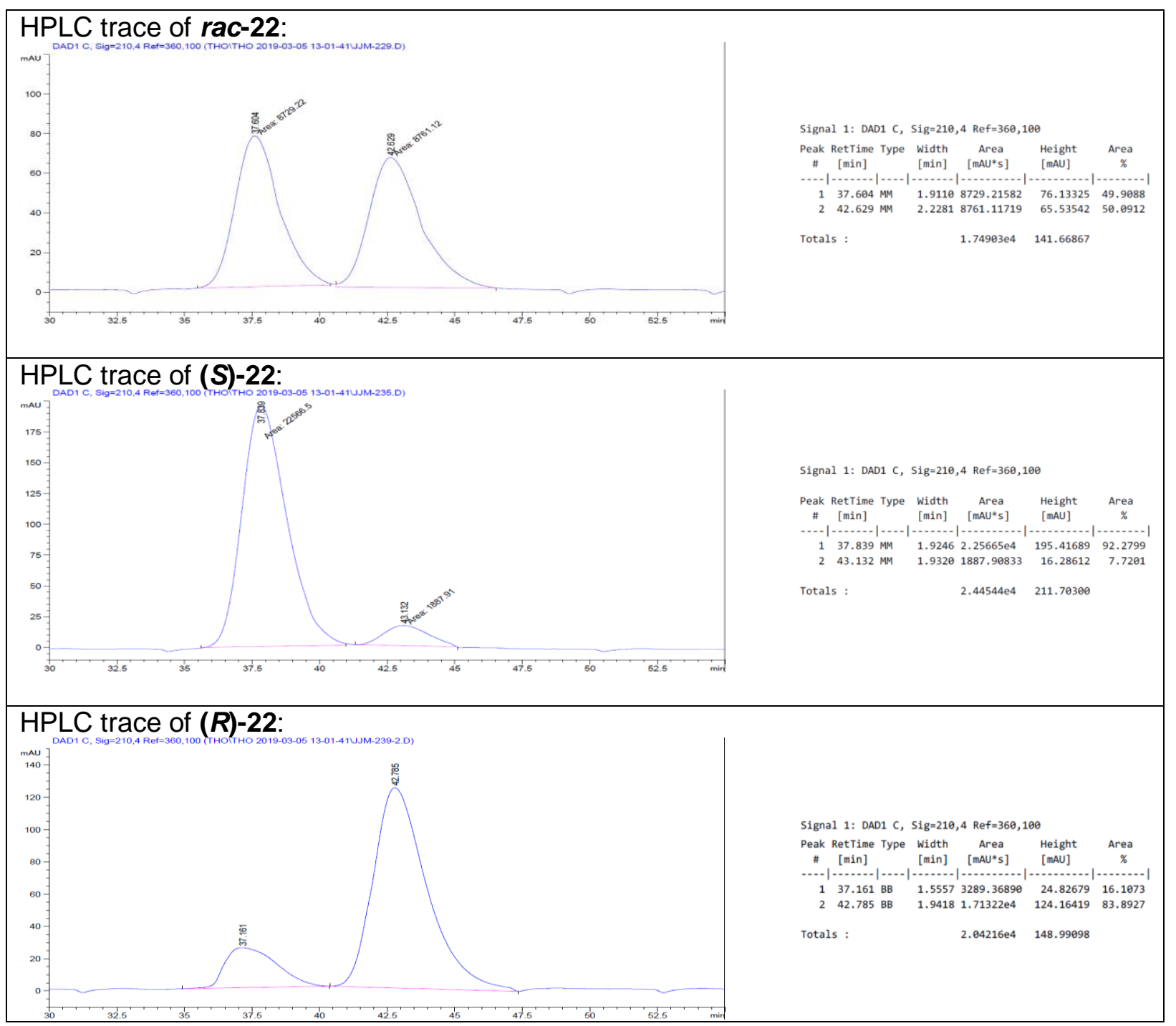

Figure 15: HPLC traces of 22; top: rac-22; middle: (S)-22 ; bottom: (R)-22. 
Synthesis of $(R)$-Lorcaserin $((R)-28)$

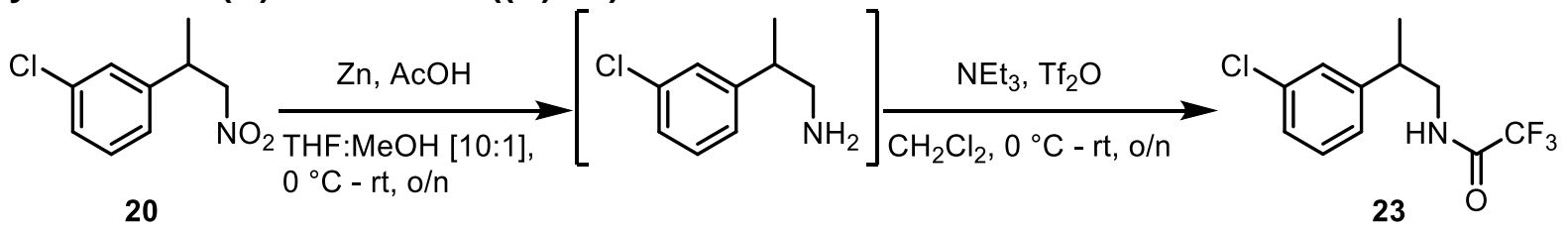

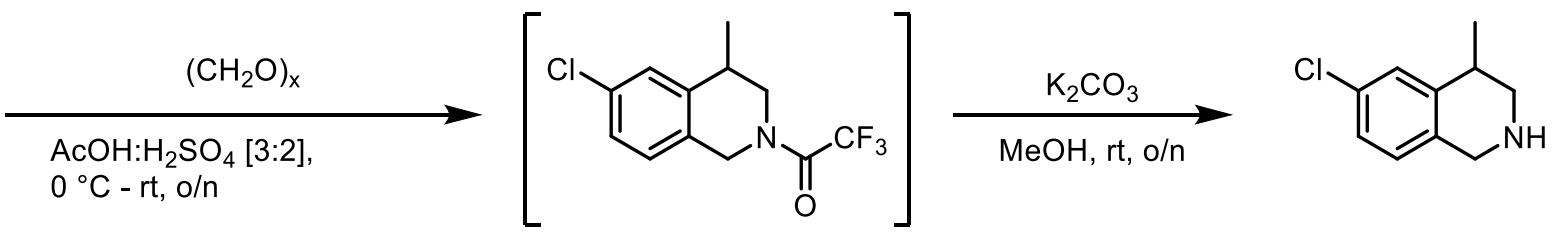

S7

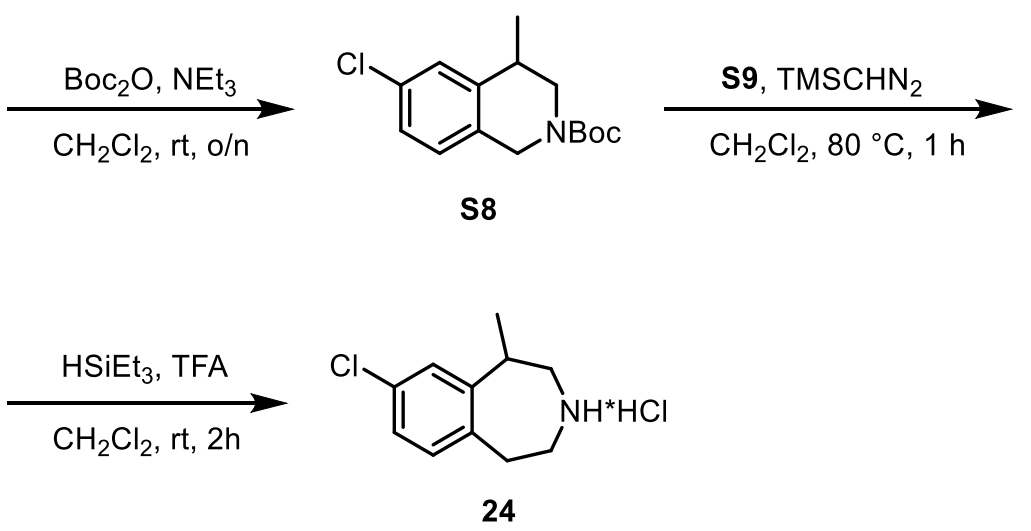<smiles>CC1CN(C(=O)OCc2ccccc2)C=Cc2ccc(Cl)cc21</smiles><smiles>CC1(C)C2CC3CC(C2)CC1(Br)C3</smiles>

Scheme 5: Preparation of racemic and $(R)$-Lorcaserin $((\boldsymbol{R})-\mathbf{2 4})$.

General procedure $\mathrm{H}$ for the synthesis of $\mathrm{N}$-(2-(3-Chlorophenyl)propyl)-2,2,2trifluoroacetamide (23)

Following an adapted literature procedure, ${ }^{[7]} 20$ (1.0 eq.) was dissolved in THF:MeOH (10:1, $0.25 \mathrm{M}$ ) and cooled to $0{ }^{\circ} \mathrm{C}$ before $\mathrm{Zn}$ dust (5 eq.) and $\mathrm{AcOH}$ (30 eq.) were added. The ice bath was removed and the reaction mixture was stirred at room temperature for $24 \mathrm{~h}$. $\mathrm{Zn}$ was removed by filtration and the residue was diluted with $\mathrm{H}_{2} \mathrm{O}$. The $\mathrm{pH}$ of the filtrate was adjusted to $\mathrm{pH} 11$ with $\mathrm{NaOH}(5 \mathrm{M})$ and was extracted with EtOAc $(3 \mathrm{x})$. The combined organic layers were washed with brine, dried over $\mathrm{Na}_{2} \mathrm{SO}_{4}$, filtered and concentrated in vacuo to yield the crude amine which was used for the next step without further analysis and purification. The amine from the previous step (1.0 eq.) was dissolved in $\mathrm{CH}_{2} \mathrm{Cl}_{2}(0.12 \mathrm{M})$, cooled to $0{ }^{\circ} \mathrm{C}$ before $\mathrm{NEt}_{3}$ (1.13 eq.) and trifluoroacetic anhydride (1.65 eq.) were added slowly. The reaction mixture was stirred over night while warming up to room temperature. After this time the reaction mixture was poured onto $\mathrm{H}_{2} \mathrm{O}$ and the separated aqueous layer was extracted with $\mathrm{CH}_{2} \mathrm{Cl}_{2}(3 \mathrm{x})$. The combined organic layers were dried over $\mathrm{Na}_{2} \mathrm{SO}_{4}$, filtered and concentrated in vacuo. Purification by column chromatography $\left(\mathrm{SiO}_{2}\right.$, EtOAc: cyclohexane) yielded the trifluoroacetamide.

\section{rac-N-(2-(3-Chlorophenyl)propyl)-2,2,2-trifluoroacetamide (rac-23)}

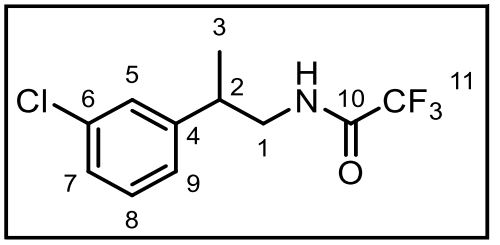

Prepared according to general procedure $\mathbf{H}$, rac-20 (405 mg, $2.03 \mathrm{mmol}, 1$ eq.) in THF:MeOH (10:1, $8.2 \mathrm{~mL})$ was reduced to the amine (321.0 $\mathrm{mg}, 1.89 \mathrm{mmol}, 93 \%)$ using Zn dust (663 $\mathrm{mg}$, $10.14 \mathrm{mmol}, 5$ eq.) and $\mathrm{AcOH}$ (3.48 mL, $60.86 \mathrm{mmol}, 30$ eq.). The crude amine (236 mg, $1.39 \mathrm{mmol}, 1.00$ eq.) was treated with $\mathrm{NEt}_{3}(0.22 \mathrm{~mL}, 1.57 \mathrm{mmol}, 1.13$ eq. $)$ and trifluoroacetic anhydride (0.32 mL, $2.30 \mathrm{mmol}, 1.65$ eq.) in $\mathrm{CH}_{2} \mathrm{Cl}_{2}(12 \mathrm{~mL})$ to yield rac-23 as a light yellow oil 
$(157.0 \mathrm{mg}, \quad 0.59 \mathrm{mmol}, 43 \%)$ after purification by column chromatography $\left(\mathrm{SiO}_{2}\right.$, EtOAc:cyclohexane, 5:95).

(R)-N-(2-(3-Chlorophenyl)propyl)-2,2,2-trifluoroacetamide ((R)-23)

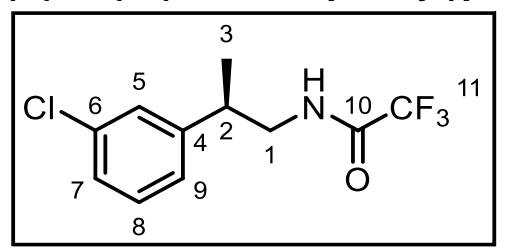
Prepared according to general procedure $\mathbf{H},(\boldsymbol{R})-\mathbf{2 0}$ (er 95:05, $349 \mathrm{mg}, 1.75 \mathrm{mmol}$, 1. eq.) in THF:MeOH (10:1, $7 \mathrm{~mL})$ was reduced to the amine (288 $\mathrm{mg}, 1.70 \mathrm{mmol}, 97 \%)$ using $\mathrm{Zn}$ dust (572 mg, $8.75 \mathrm{mmol}$, 5 eq.) and $\mathrm{AcOH}(3 \mathrm{~mL}, 52.5 \mathrm{mmol}$, 30 eq.). The crude amine ( $288 \mathrm{mg}, 1.70 \mathrm{mmol}, 1.00$ eq.) was treated with $\mathrm{NEt}_{3}(0.27 \mathrm{~mL}, 1.92 \mathrm{mmol}, 1.13$ eq. $)$ and trifluoroacetic anhydride $(0.39 \mathrm{~mL}, 2.81 \mathrm{mmol}, 1.65$ eq. $)$ in $\mathrm{CH}_{2} \mathrm{Cl}_{2}(8 \mathrm{~mL})$ to yield $(\boldsymbol{R})-23$ as a light yellow oil ( $372.9 \mathrm{mg}, 1.40 \mathrm{mmol}, 83 \%)$ after purification by column chromatography $\left(\mathrm{SiO}_{2}\right.$, EtOAc:cyclohexane, 5:95). The enantiomeric ratio was found to be 95:05 by chiral HPLC (Reprosil Chiral-OM, $n$-hexane: $\mathrm{PrOH}$ 97:03, $1.0 \mathrm{~mL} / \mathrm{min}, \lambda=210 \mathrm{~nm}$, $\mathrm{t}_{\mathrm{R}}$ (major): $11.640 \mathrm{~min}$, $t_{R}$ (minor): $14.254 \mathrm{~min}$ ).

(R)-23 (er 95:05): [a] $]_{D}^{25}=+54.2\left(\mathrm{c}=0.5\right.$ in $\left.\mathrm{CHCl}_{3}\right)$; $\mathbf{R}_{\boldsymbol{f}}=0.21$ (EtOAc:cyclohexane 5:95); ${ }^{1} \mathrm{H}-\mathrm{NMR}\left(600 \mathrm{MHz}, \mathrm{CDCl}_{3}\right): \delta=7.30-7.24(\mathrm{~m}, 2 \mathrm{H}, \mathrm{H} 8, \mathrm{H} 7), 7.20\left(\mathrm{t},{ }^{4} \mathrm{~J}_{\mathrm{HH}}=1.9 \mathrm{~Hz}, 1 \mathrm{H}, \mathrm{H} 5\right)$, $7.09-7.07(\mathrm{~m}, 1 \mathrm{H}, \mathrm{H} 9), 6.13(\mathrm{~s}, 1 \mathrm{H}, \mathrm{NH}), 3.69-3.63(\mathrm{~m}, 1 \mathrm{H}, \mathrm{H} 1), 3.39-3.31\left(\mathrm{~m}, 1 \mathrm{H}, \mathrm{H} 1^{\prime}\right)$, $3.06-2.97(\mathrm{~m}, 1 \mathrm{H}, \mathrm{H} 2), 1.31\left(\mathrm{~d},{ }^{3} \mathrm{JHH}_{\mathrm{HH}}=7.0 \mathrm{~Hz}, 3 \mathrm{H}, \mathrm{H} 3\right) \mathrm{ppm} ;{ }^{13} \mathrm{C}\left\{{ }^{1} \mathrm{H}\right\}-\mathrm{NMR}\left(151 \mathrm{MHz}, \mathrm{CDCl}_{3}\right)$ : $\delta=157.4\left(q,{ }^{2} J_{\mathrm{CF}}=36.5 \mathrm{~Hz}, \mathrm{C} 10\right), 145.0$ (C4), 135.0 (C6), 130.4 (C8), 127.6 (C7), 127.3 (C5), 125.5 (C9), 115.9 (q, $\left.{ }^{1} J_{\mathrm{CF}}=287.8 \mathrm{~Hz}, \mathrm{C} 11\right), 46.3$ (C1), $39.3(\mathrm{C} 2), 18.9$ (C3) ppm; ${ }^{19} \mathrm{~F}-\left\{{ }^{1} \mathrm{H}\right\}-\mathrm{NMR}$ (564 MHz, $\left.\mathrm{CDCl}_{3}\right): \delta=-76.01(\mathrm{~s})$ ppm; IR (ATR): $\tilde{v}=3305(\mathrm{w}), 3105(\mathrm{w}), 2972(\mathrm{w}), 1702(\mathrm{~s})$, $1598(w), 1558(w), 1479(w), 1457(w), 1433(w), 1384(w), 1365(w), 1338(w), 1205(m), 1154$ (s), 1084 (w), 999 (w), 931 (w), 880 (w), $836(w), 784(\mathrm{~m}), 725(\mathrm{~m}), 696(\mathrm{~s}) \mathrm{cm}^{-1}$; HR-ESI-MS: $\mathrm{m} / \mathrm{z}$ : $[\mathrm{M}+\mathrm{Na}]^{+}$calcd. for $\mathrm{C}_{11} \mathrm{H}_{11} \mathrm{NOClF}_{3} \mathrm{Na}^{+}: 288.0378$; found: 288.0380 . The analytical data are in agreement with the literature. ${ }^{[7]}$

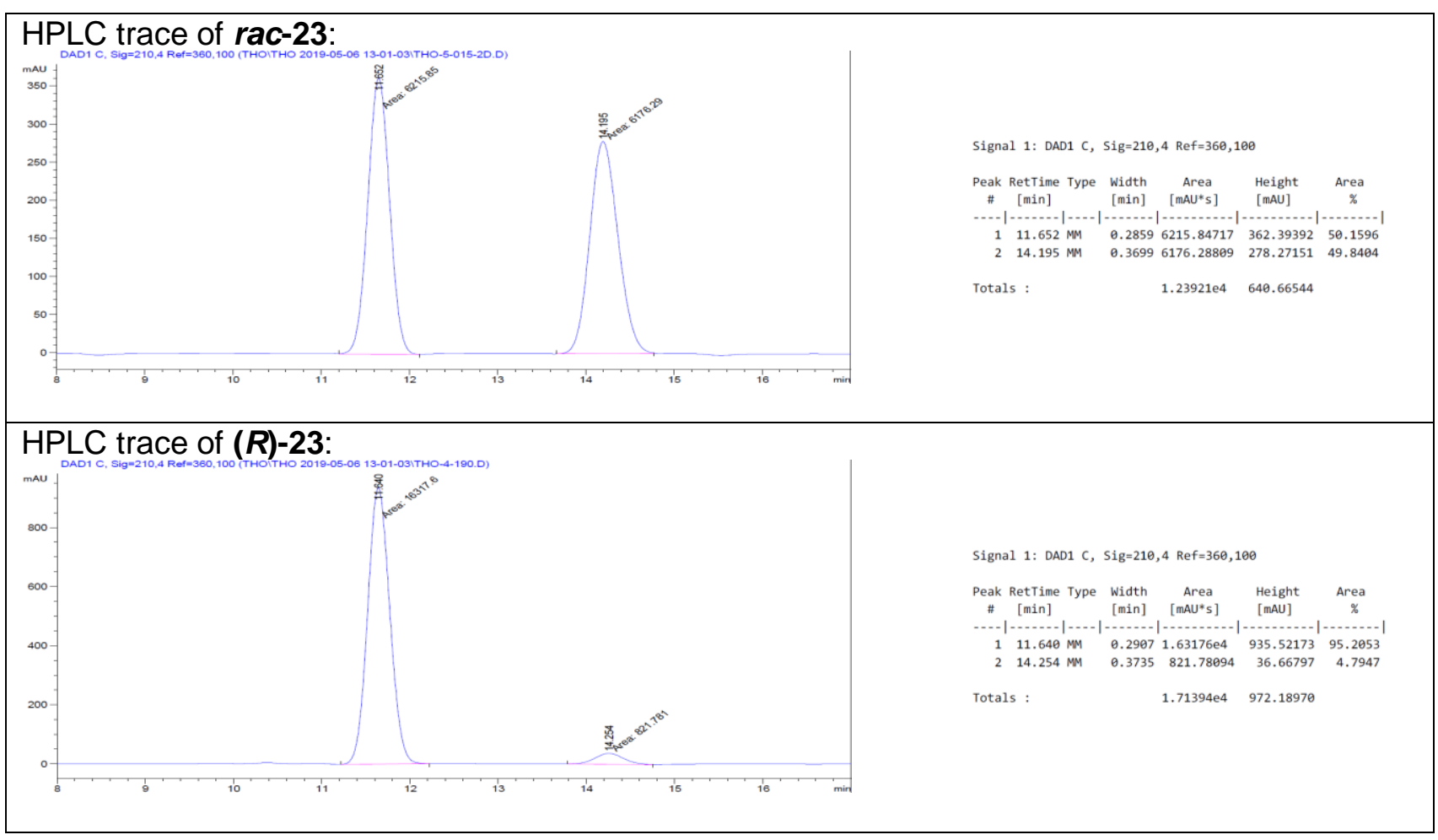

Figure 16: HPLC traces of 23; top: rac-23; bottom: $(\boldsymbol{R}) \mathbf{- 2 3}$. 
General procedure I for the synthesis of 6-chloro-4-methyl-1,2,3,4-tetrahydroisoquinoline (S7)

Following an adapted literature procedure, ${ }^{[7]} \mathrm{AcOH}(0.23 \mathrm{M})$ and $\mathrm{H}_{2} \mathrm{SO}_{4}(0.35 \mathrm{M})$ were mixed at $0{ }^{\circ} \mathrm{C}$ before 23 ( 1 eq.) and paraformaldehyde (2 eq.) were added sequentially. The reaction mixture was stirred at room temperature over night and then poured onto $\mathrm{H}_{2} \mathrm{O}$. After extraction with $\mathrm{EtOAc}(3 \mathrm{x})$, the combined organic layers were washed with a sat. aq. $\mathrm{NaHCO}_{3}$ solution, $\mathrm{H}_{2} \mathrm{O}$ and brine, dried over $\mathrm{Na}_{2} \mathrm{SO}_{4}$, filtered and concentrated in vacuo. The crude trifluoroacetate protected tetrahydroisoquinoline was used without further analysis and purification for the next step. The crude trifluoroacetate protected tetrahydro-isoquinoline $(1.0$ eq.) from the previous step was dissolved in $\mathrm{MeOH}(0.1 \mathrm{M})$ and an aqueous $\mathrm{K}_{2} \mathrm{CO}_{3}$ solution $(0.44 \mathrm{M}, 3$ eq.) was added. The reaction mixture was stirred at room temperature overnight before being acidified to $\mathrm{pH} 8$ with $\mathrm{HCl}(1 \mathrm{M})$. This mixture was extracted with EtOAc $(3 \mathrm{x})$ and the combined organic layers were washed with $\mathrm{H}_{2} \mathrm{O}(2 \mathrm{x})$, dried over $\mathrm{Na}_{2} \mathrm{SO}_{4}$, filtered and concentrated in vacuo. Purification by column chromatography $\left(\mathrm{SiO}_{2}, \mathrm{MeOH}: \mathrm{EtOAc}\right)$ yielded $\mathbf{S 7}$.

\section{rac-6-chloro-4-methyl-1,2,3,4-tetrahydroisoquinoline (rac-S7)}

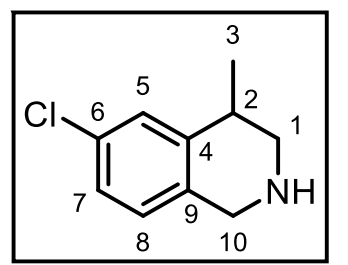

Prepared according to general procedure I, rac-23 $(133 \mathrm{mg}, 0.50 \mathrm{mmol}$, 1 eq.) and paraform-aldehyde (30 $\mathrm{mg}, 1.00 \mathrm{mmol}, 2$ eq.) were reacted in $\mathrm{AcOH}(2.2 \mathrm{~mL})$ and $\mathrm{H}_{2} \mathrm{SO}_{4}(1.4 \mathrm{~mL})$ to the crude trifluoroacetate protected tetrahydroisoquinoline, a light yellow oil $(94.1 \mathrm{mg}, 0.34 \mathrm{mmol}, 68 \%)$. This trifluoroacetate protected tetrahydroisoquinoline $(77 \mathrm{mg}, 0.28 \mathrm{mmol}$, 1 eq.) was deprotected using an aqueous $\mathrm{K}_{2} \mathrm{CO}_{3}$ solution $(0.44 \mathrm{M}, 1.9 \mathrm{~mL}$, $0.84 \mathrm{mmol}, 3$ eq.) and $\mathrm{MeOH}(3.3 \mathrm{~mL})$. Purification by column chromatography $\left(\mathrm{SiO}_{2}, \mathrm{MeOH}\right.$ :EtOAc $\left.0: 1-1: 1\right)$ yielded rac-24 as a yellow solid $(16.0 \mathrm{mg}$, $0.09 \mathrm{mmol}, 18 \%)$.

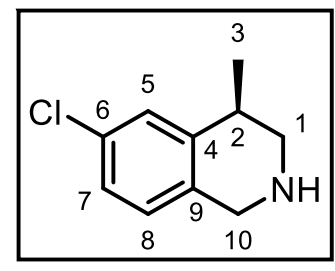

(R)-6-chloro-4-methyl-1,2,3,4-tetrahydroisoquinoline ((R)-S7)

Prepared according to general procedure I, $(\boldsymbol{R})-\mathbf{2 3}$ (er 95:05, $310 \mathrm{mg}$, $1.17 \mathrm{mmol}, 1$ eq.) and paraformaldehyde $(21 \mathrm{mg}, 0.70 \mathrm{mmol}, 2$ eq.) were reacted in $\mathrm{AcOH}(1.5 \mathrm{~mL})$ and $\mathrm{H}_{2} \mathrm{SO}_{4}(1 \mathrm{~mL})$ to the crude trifluoroacetate protected tetrahydroisoquinoline, a light yellow oil $(320.7 \mathrm{mg}, 1.15 \mathrm{mmol}$, $99 \%$ ). This crude tetrafluoroacetate protected tetrahydroisoquinoline (320 mg, $1.15 \mathrm{mmol}$, 1 eq.) was deprotected using an aqueous $\mathrm{K}_{2} \mathrm{CO}_{3}$ solution ( $0.44 \mathrm{M}, 8 \mathrm{~mL}, 3.51 \mathrm{mmol}, 3 \mathrm{eq}$.) and $\mathrm{MeOH}(14 \mathrm{~mL})$. Purification by column chromatography $\left(\mathrm{SiO}_{2}, \mathrm{MeOH}\right.$ :EtOAc $\left.0: 1-1: 1\right)$ yielded $(\boldsymbol{R})$-S7 as viscuous yellow oil $(90.1 \mathrm{mg}, 0.50 \mathrm{mmol}, 43 \%)$.

$(\boldsymbol{R})-23:[\alpha]_{D}{ }^{25}=+35.5\left(\mathrm{c}=0.5\right.$ in $\left.\mathrm{CHCl}_{3}\right) ; \mathbf{R}_{\boldsymbol{f}}=0.27\left(\mathrm{MeOH}:\right.$ EtOAc 20:80); ${ }^{1} \mathrm{H}-\mathrm{NMR}(600 \mathrm{MHz}$, $\left.\mathrm{CDCl}_{3}\right): \delta=7.22(\mathrm{~s}, 1 \mathrm{H}, \mathrm{H} 5), 7.14\left(\mathrm{~d},{ }^{3} \mathrm{JHH}_{\mathrm{HH}}=8.1 \mathrm{~Hz}, 1 \mathrm{H}, \mathrm{H} 7\right), 6.98\left(\mathrm{~d},{ }^{3} \mathrm{~J}_{\mathrm{HH}}=8.1 \mathrm{~Hz}, 1 \mathrm{H}, \mathrm{H} 8\right)$, 5.53 (br s, 1H, NH), 4.10 (s, 2H, H10), $3.33\left(\mathrm{~d},{ }^{2} J_{\mathrm{HH}}=12.5 \mathrm{~Hz}, 1 \mathrm{H}, \mathrm{H} 1 / 1{ }^{\prime}\right), 3.11-3.03(\mathrm{~m}, 1 \mathrm{H}$, $\mathrm{H} 2), 2.88-2.82\left(\mathrm{~m}, 1 \mathrm{H}, \mathrm{H} 1 / 1{ }^{\prime}\right), 1.33\left(\mathrm{~d},{ }^{3} \mathrm{JHH}_{\mathrm{HH}}=7.2 \mathrm{~Hz}, 3 \mathrm{H}, \mathrm{H} 3\right) \mathrm{ppm} ;{ }^{13} \mathrm{C}\left\{{ }^{1} \mathrm{H}\right\}-\mathrm{NMR}(151 \mathrm{MHz}$, $\left.\mathrm{CDCl}_{3}\right): \delta=140.6$ (C4), 133.0 (C6), 130.3 (C9), 127.9 (C8), 127.9 (C5), 126.8 (C7), 49.2 (C1), 46.4 (C10), 31.0 (C2), 19.7 (C3) ppm; IR (ATR): $\tilde{v}=2932$ (w), $2862(w), 2749$ (w), $2670(w)$, 2590 (w), 2470 (w), 2429 (w), $1679(w), 1601(w), 1573(w), 1483(m), 1448(s), 1405(w), 1383$ $(w), 1351(w), 1332(w), 1271(w), 1222(w), 1190(w), 1132(w), 1096(w), 1082(w), 1028(w)$, $971(w), 961(w), 917(w), 880(w), 857(w), 829(s), 808(w), 779(w), 730(w), 698(w), 662$ (m) $\mathrm{cm}^{-1}$; HR-ESI-MS: $\mathrm{m} / \mathrm{z}$ : $[\mathrm{M}+\mathrm{H}]^{+}$calcd. for $\mathrm{C}_{10} \mathrm{H}_{12} \mathrm{NClH}^{+}: 182.0731$; found: 182.0739 .

\section{General procedure $\mathrm{J}$ for the synthesis of tert-butyl-6-chloro-4-methyl-3,4-dihydro- isoquinoline-2(1H)-carboxylate (S8)}

Following an adapted literature procedure, ${ }^{[7]} \mathbf{S 7}$ (1.0 eq.) was dissolved in $\mathrm{CH}_{2} \mathrm{Cl}_{2}(0.03 \mathrm{M}$ ) and $\mathrm{Boc}_{2} \mathrm{O}$ (1.1 eq.) and $\mathrm{NEt}_{3}$ (1.2 eq.) were added. The reaction mixture was stirred at room temperature over night, before $\mathrm{H}_{2} \mathrm{O}$ was added. The aqueous layer was extracted with $\mathrm{CH}_{2} \mathrm{Cl}_{2}$ $(3 \mathrm{x})$ and the combined organic layers were washed with an aq. $\mathrm{NaOH}$ solution $(1 \mathrm{M})$, brine, dried over $\mathrm{Na}_{2} \mathrm{SO}_{4}$, filtered and concentrated in vacuo. The carboxylate was used for the next step without further purification. 
rac-tert-Butyl-6-chloro-4-methyl-3,4-dihydroisoquinoline-2(1H)-carboxylate (rac-S8)

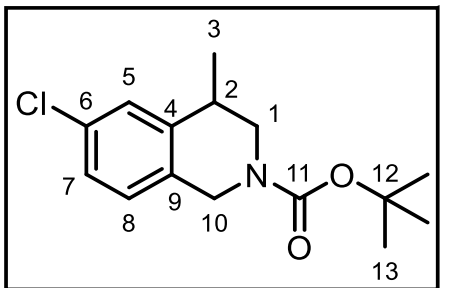

Prepared according to general procedure J, rac-S7 $(15 \mathrm{mg}$, $0.08 \mathrm{mmol}, 1.0$ eq.) was Boc-protected using $\mathrm{Boc}_{2} \mathrm{O}(25 \mathrm{mg}$, $0.09 \mathrm{mmol}, 1.1$ eq.) and $\mathrm{NEt}_{3}$ (3 drops) in $\mathrm{CH}_{2} \mathrm{Cl}_{2}(3 \mathrm{~mL})$. The carboxylate was obtained as a yellow oil $(21.0 \mathrm{mg}, 0.08 \mathrm{mmol}$, $93 \%$ ) and used without further purification.

tert-Butyl (R)-6-chloro-4-methyl-3,4-dihydroisoquinoline-2(1H)-carboxylate $((R)$-S8)

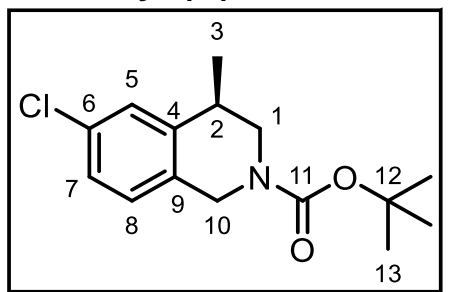

Prepared according to general procedure $\mathbf{J},(\boldsymbol{R})-\mathbf{S} 7$ (70 mg, $0.38 \mathrm{mmol}, 1.0$ eq.) was Boc-protected using $\mathrm{Boc}_{2} \mathrm{O}$ (119 mg, $0.42 \mathrm{mmol}, 1.1$ eq.) and $\mathrm{NEt}_{3}(0.06 \mathrm{~mL}, 0.46 \mathrm{mmol}, 1.2$ eq.) in $\mathrm{CH}_{2} \mathrm{Cl}_{2}(15 \mathrm{~mL})$. The carboxylate was obtained as a yellow oil $(63.7 \mathrm{mg}, \quad 0.23 \mathrm{mmol}, 59 \%)$ after purification by column chromatography $\quad\left(\mathrm{SiO}_{2}\right.$, EtOAc:cyclohexane 5:95). The enantiomeric ratio was found to be 95:05 by chiral HPLC (Reprosil Chiral-OM, $n$-hexane: $: P r O H$ 99.3:0.7, $1.0 \mathrm{~mL} / \mathrm{min}, \lambda=210 \mathrm{~nm}, \mathrm{t}_{\mathrm{R}}$ (major): $7.022 \mathrm{~min}, \mathrm{t}_{\mathrm{R}}$ (minor): $7.452 \mathrm{~min})$.

$(\boldsymbol{R})-25$ (er 95:05): [a $]_{\mathrm{D}}{ }^{25}=-18\left(\mathrm{c}=0.1\right.$ in $\left.\mathrm{CHCl}_{3}\right) ; \mathbf{R}_{f}=0.34$ (EtOAc:cyclohexane 5:95); ${ }^{\mathbf{1}} \mathbf{H}-\mathrm{NMR}$ $\left(500 \mathrm{MHz}, \mathrm{CDCl}_{3}\right): \delta=7.18(\mathrm{~s}, 1 \mathrm{H}, \mathrm{H} 5), 7.16-7.13(\mathrm{~m}, 1 \mathrm{H}, \mathrm{H} 7), 7.02\left(\mathrm{~d},{ }^{3} \mathrm{~J}_{\mathrm{HH}}=8.1 \mathrm{~Hz}, 1 \mathrm{H}\right.$, H8), 4.69 (br s, 1H, H10), $4.48-4.31\left(\mathrm{~m}, 1 \mathrm{H}, \mathrm{H} 10^{\prime}\right), 3.52$ (br s, 2H, H1), $3.00-2.86(\mathrm{~m}, 1 \mathrm{H}$, $\mathrm{H} 2), 1.49(\mathrm{~s}, 9 \mathrm{H}, \mathrm{H} 13), 1.26\left(\mathrm{~d},{ }^{3} \mathrm{~J}_{\mathrm{HH}}=7.0 \mathrm{~Hz}, 3 \mathrm{H}, \mathrm{H} 3\right) \mathrm{ppm} ;{ }^{13} \mathbf{C}\left\{{ }^{1} \mathrm{H}\right\}-\mathrm{NMR}\left(126 \mathrm{MHz}, \mathrm{CDCl}_{3}\right):$ $\delta=155.2$ (C11), 142.0 (C4), 132.3 (C6), 131.7 (C9), 127.7 (C5), 127.6 (C8), 126.5 (C7), 80.1 (C12), 47.6 (C1), 45.5 (C10), 33.2 (C2), 28.6 (C13), 19.5 (C3) ppm; IR (ATR): $\tilde{v}=2978(w)$, 2933 (w), 1807 (w), $1757(w), 1694(m), 1600(w), 1573(w), 1478(w), 1458(w), 1419(w), 1369$ (w), $1327(w), 1307(w), 1236(w), 1211(m), 1162(m), 1114(s), 1065(s), 1012(w), 940(w)$, $914(\mathrm{w}), 874(\mathrm{w}), 844(\mathrm{~m}), 818(\mathrm{w}), 774(\mathrm{w}), 706(\mathrm{w}) \mathrm{cm}^{-1}$; HR-ESI-MS: $\mathrm{m} / \mathrm{z}$ : [M+Na] ${ }^{+}$calcd. for $\mathrm{C}_{15} \mathrm{H}_{20} \mathrm{NO}_{2} \mathrm{CINa}^{+}$: 304.1075; found: 304.1089. The analytical data are in agreement with the literature. ${ }^{[7]}$

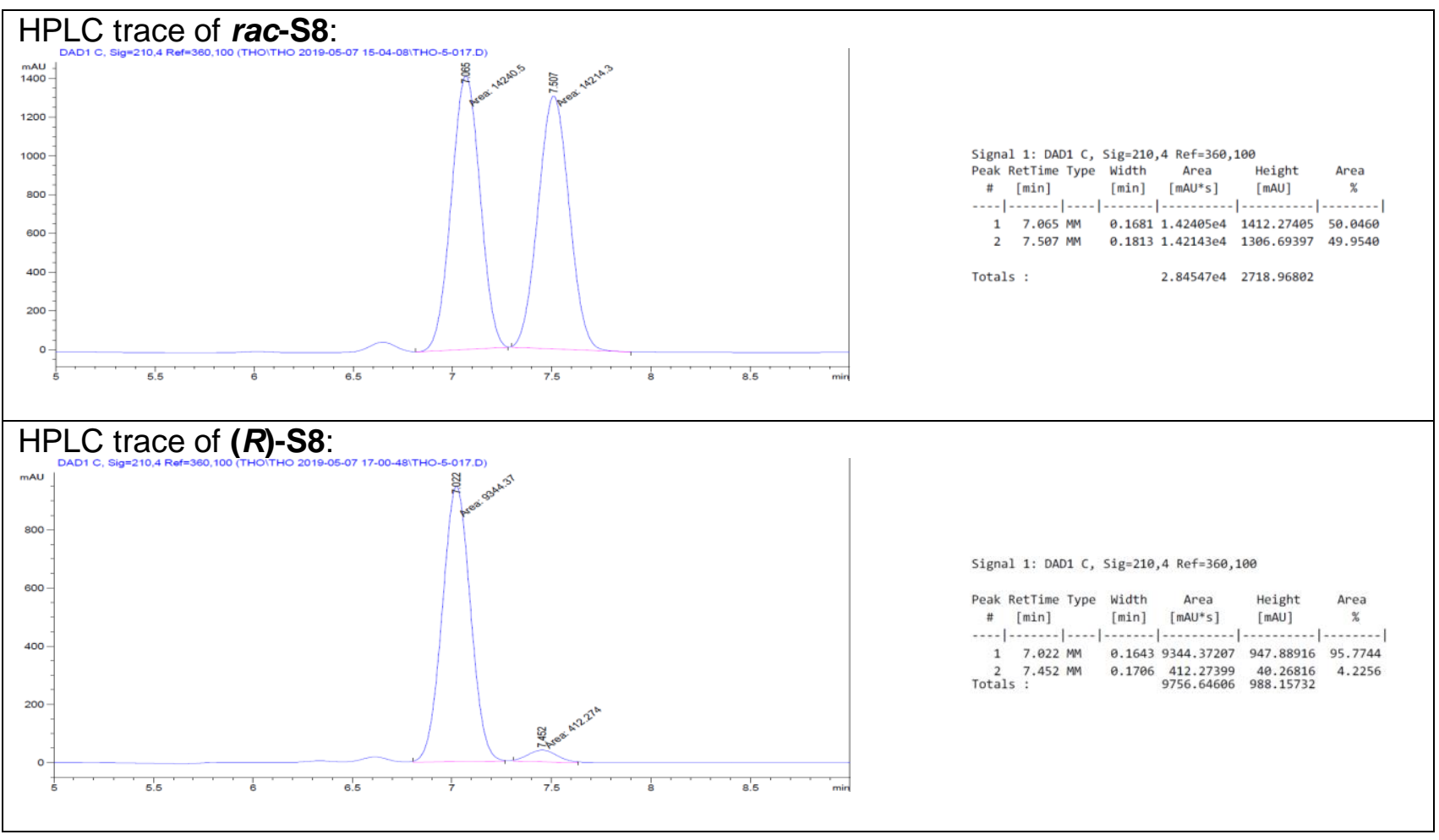

Figure 17: HPLC traces of S8; top: rac-S8; bottom: $(\boldsymbol{R})-\mathbf{S 8 .}$ 
2,2,6,6-Tetramethylpiperidine-1-oxoammonium tetrafluoroborate (S9)

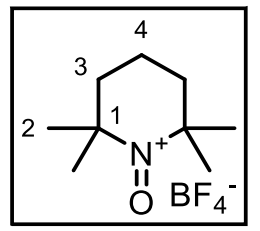

Following an adapted literature procedure, ${ }^{[19]}$ TEMPO $(1.78 \mathrm{~g}, 11.4 \mathrm{mmol}$, 1.0 eq.) was suspended in $\mathrm{H}_{2} \mathrm{O}(6 \mathrm{~mL})$ and $\mathrm{HBF}_{4}\left(48 \%\right.$ in $\mathrm{H}_{2} \mathrm{O}, 1.75 \mathrm{~mL}$, $13.1 \mathrm{mmol}, 1.15$ eq.) was added over $15 \mathrm{~min}$ via addition funnel. The mixture was stirred at room temperature for $30 \mathrm{~min}$ before being cooled to $0{ }^{\circ} \mathrm{C}$. At that temperature $\mathrm{NaOCl}\left(11-15 \%\right.$ in $\mathrm{H}_{2} \mathrm{O}, 3.9 \mathrm{~mL}, 5.7 \mathrm{mmol}, 0.5$ eq.) was added via an addition funnel over 30 min and the resulting mixture was stirred for further $60 \mathrm{~min}$. The resulting yellow suspension was filtered and the yellow solid was washed with ice cold $\mathrm{H}_{2} \mathrm{O}(4 \times 5 \mathrm{~mL})$ and $\mathrm{CH}_{2} \mathrm{Cl}_{2}(3 \times 5 \mathrm{~mL})$. After drying at the lyophiliser over night $\mathbf{S 9}$ was obtained as a yellow powder (1.31 g, $5.37 \mathrm{mmol}, 47 \%)$.

${ }^{1} \mathrm{H}-\mathrm{NMR}\left(500 \mathrm{MHz}, \mathrm{CD}_{3} \mathrm{CN}\right): \delta=2.50$ (br s, 4H, H3), 2.41 (br s, 2H, H4), 1.66 (s, 12H, H2) ppm; ${ }^{13} \mathrm{C}\left\{{ }^{1} \mathrm{H}\right\}-N M R\left(126 \mathrm{MHz}, \mathrm{CD}_{3} \mathrm{CN}\right)$ : no signals; ${ }^{19} \mathrm{~F}\left\{{ }^{1} \mathrm{H}\right\}-\mathrm{NMR}\left(470 \mathrm{MHz}, \mathrm{CD}_{3} \mathrm{CN}\right): \delta=-151.59$ (s), -151.64 (s) ppm; ${ }^{11} \mathbf{B}-\left\{{ }^{1} \mathbf{H}\right\}-N M R(96 \mathrm{MHz}): \delta=-1.21 \mathrm{ppm}$; IR (ATR): $\tilde{v}=2943(\mathrm{w}), 1627(\mathrm{~m})$, 1473 (w), 1400 (w), 1382 (w), 1290 (w), 1242 (w), 1212 (w), 1098 (m), 1062 (s), 975 (m), 899 (w), 878 (w), 858 (w), 765 (w), 705 (w) cm ${ }^{-1}$; HR-ESI-MS: $\mathrm{m} / \mathrm{z}$ : [M-BF$\left.{ }_{4}^{-}\right]^{+}$calcd. for $\mathrm{C}_{9} \mathrm{H}_{18} \mathrm{NO}^{+}$: 156.13829; found: 156.13816 ; m.p.: $=147-148^{\circ} \mathrm{C}$ (decomposition). The analytical data are in agreement with the literature. ${ }^{[19]}$

General procedure $\mathrm{K}$ for the synthesis of tert-butyl-8-chloro-1-methyl-1,2-dihydro-3Hbenzo[d]azepine-3-carboxylate (S10)

Following an adapted literature procedure, ${ }^{[7]} \mathbf{S 8}$ (1 eq.) was dissolved in dry $\mathrm{CH}_{2} \mathrm{Cl}_{2}(0.1 \mathrm{M})$ in a flame dried Schlenk pressure tube under an argon atmosphere. S9 (1.5 eq.) was added followed by dropwise addition of $\mathrm{TMSCHN}_{2}\left(2 \mathrm{M}\right.$ in $\mathrm{Et}_{2} \mathrm{O}, 2.4$ eq.). The reaction mixture was stirred at $80^{\circ} \mathrm{C}$ for $1 \mathrm{~h}$. After removal of the solvent and purification by column chromatography $\left(\mathrm{SiO}_{2}, \mathrm{Et}_{2} \mathrm{O}: n\right.$-pentane) $\mathbf{S 1 0}$ was obtained as a yellow oil.

rac-tert-Butyl-8-chloro-1-methyl-1,2-dihydro-3H-benzo[d]azepine-3-carboxylate

(racS10)

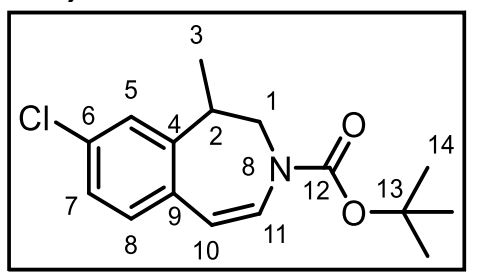

Prepared according to general procedure K, rac-S8 $(52 \mathrm{mg}$, $0.18 \mathrm{mmol}, \quad 1.0$ eq.), $\mathbf{S 9}(67 \mathrm{mg}, 0.28 \mathrm{mmol}, 1.5$ eq.) and $\mathrm{TMSCHN}_{2}$ (2 $\mathrm{M}$ in $\mathrm{Et}_{2} \mathrm{O}, 0.22 \mathrm{~mL}, 0.44 \mathrm{mmol}, 2.4$ eq.) were reacted in $\mathrm{CH}_{2} \mathrm{Cl}_{2}(1.8 \mathrm{~mL})$ to the crude benzo[d]azepine carboxylate. Purification by column chromatography $\left(\mathrm{SiO}_{2}\right.$, $\mathrm{Et}_{2} \mathrm{O}: n$-pentane $\left.3: 97\right)$ yielded rac-S10 as a yellow oil $(16.6 \mathrm{mg}$, $0.06 \mathrm{mmol}, 31 \%)$.

\section{tert-Butyl $(R)$-8-chloro-1-methyl-1,2-dihydro-3H-benzo[d]-azepine-3-carboxylate}

$((R)-\mathrm{S} 10)$

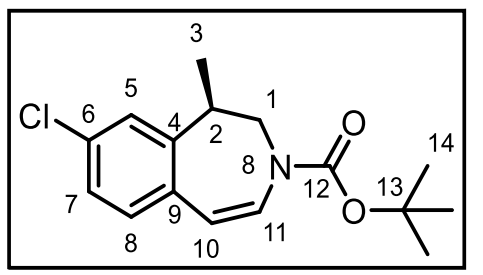

Prepared according to general procedure K, $(\boldsymbol{R})$-S8 (er 95:05, $56 \mathrm{mg}, 0.20 \mathrm{mmol}, 1 \mathrm{eq}.), \mathbf{S 9}$ (78 $\mathrm{mg}, 0.30 \mathrm{mmol}, 1.5$ eq.) and $\mathrm{TMSCHN}_{2}\left(2 \mathrm{M}\right.$ in $\mathrm{Et}_{2} \mathrm{O}, 0.24 \mathrm{~mL}, 0.48 \mathrm{mmol}, 2.4$ eq.) were reacted in $\mathrm{CH}_{2} \mathrm{Cl}_{2}(2.0 \mathrm{~mL})$ to the crude benzo[d]azepine carboxylate. Purification by column chromatography $\left(\mathrm{SiO}_{2}\right.$, $\mathrm{Et}_{2} \mathrm{O}: n$-pentane $\left.1: 99\right)$ yielded $(\boldsymbol{R}) \mathbf{- S 1 0}$ as a yellow oil $(17.3 \mathrm{mg}$, $0.06 \mathrm{mmol}, 33 \%)$.

$\mathbf{R}_{\boldsymbol{f}}=0.54\left(\mathrm{Et}_{2} \mathrm{O}: n\right.$-pentane, 3:97); ${ }^{1} \mathrm{H}-\mathrm{NMR}\left(600 \mathrm{MHz}, \mathrm{CDCl}_{3}\right): \delta=7.13-7.07(\mathrm{~m}, 3 \mathrm{H}, \mathrm{H} 7, \mathrm{H} 8$, H5), 6.92 (br s, $1 \mathrm{H}, \mathrm{H} 11$ ), 5.58 (br s, $1 \mathrm{H}, \mathrm{H} 10), 4.59$ (br s, $\left.1 \mathrm{H}, \mathrm{H} 1 / 1^{\prime}\right), 3.28\left(\mathrm{p},{ }^{3} J_{\mathrm{HH}}=7.1 \mathrm{~Hz}, 1 \mathrm{H}\right.$, $\mathrm{H} 2), 3.11\left(\mathrm{br} \mathrm{s}, 1 \mathrm{H}, \mathrm{H} 1 / 1{ }^{\prime}\right), 1.53(\mathrm{~s}, 9 \mathrm{H}, \mathrm{H} 14), 1.10\left(\mathrm{~d},{ }^{3} \mathrm{~J}_{\mathrm{HH}}=7.1 \mathrm{~Hz}, 3 \mathrm{H}, \mathrm{H} 3\right) \mathrm{ppm} ;{ }^{13} \mathrm{C}\left\{{ }^{1} \mathrm{H}\right\}-\mathrm{NMR}$ (151 MHz, CDCl 3 ): $\delta=183.8$ (C12), 145.9 (C4), 132.5 (C9), 132.4 (C5), 131.3 (C6), 128.5 (C8), 127.6 (C11), 126.5 (C7), 107.3 (C10), 81.9 (C13), 47.3 (C1), 40.9 (C2), 28.4 (C14), 16.6 (C3) ppm; IR (ATR): $\tilde{v}=2928$ (w), $1811(w), 1754(w), 1711(w), 1638$ (w), $1595(w), 1459(w)$, $1434(\mathrm{w}), 1369(\mathrm{~m}), 1354(\mathrm{w}), 1305(\mathrm{w}), 1244(\mathrm{~m}), 1211(\mathrm{w}), 1154(\mathrm{~m}), 1116(\mathrm{~s}), 1067(\mathrm{~m}), 1011$ (w), 955 (w), 926 (w), 835 (s), 758 (w), 689 (w), 656 (w) cm $\mathrm{cm}^{-1}$ HR-ESI-MS: $\mathrm{m} / \mathrm{z}$ : [M+Na] ${ }^{+}$calcd. 
for $\mathrm{C}_{16} \mathrm{H}_{20} \mathrm{NO}_{2} \mathrm{CINa}^{+}: 316.1075$; found: 316.1082 . The analytical data are in agreement with the literature. ${ }^{[7]}$

\section{Synthesis of 8-chloro-1-methyl-2,3,4,5-tetrahydro-1 $\mathrm{H}$-benzo[d]azepine hydrochloric acid; Lorcaserin (24)}

The racemic sample of racemic Lorcaserin was synthesized via the following different two step procedure. ${ }^{[20]}$

\section{$\mathrm{N}$-(4-Chlorophenethyl)prop-2-en-1-amine hydrochloric acid (S11)}

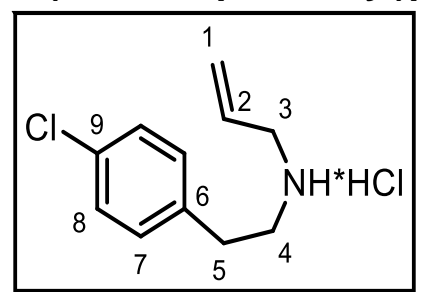

2-(4-Chlorophenyl)ethan-1-ol (5 mL, $36.9 \mathrm{~mL}, \quad 1.0$ eq.) and powdered $\mathrm{KOH}(8.29 \mathrm{~g}, 147.8 \mathrm{mmol}, 4.0 \mathrm{eq}$.) were dissolved in dry THF $(65 \mathrm{~mL})$ and cooled to $10^{\circ} \mathrm{C}$. 4-Toluenesulfonylchloride $(9.16 \mathrm{~g}$, $48.0 \mathrm{mmol}, 1.3$ eq.) was added and the reaction mixture was stirred over night while warming up to room temperature. Filtration over Celite ${ }^{\circledR}$ and concentration in vacuo yielded an orange oil which was dissolved in allylamine ( $11.1 \mathrm{~mL}, 147.8 \mathrm{mmol}, 4.0$ eq.) stirred at $50^{\circ} \mathrm{C}$ over night. The volatiles were removed in vacuo and the residue was dissolved in toluene before an aq. $\mathrm{NaOH}$ solution (2 $\mathrm{M}$ ) was added. The separated aqueous layer was extracted with toluene $(2 \mathrm{x})$ and the combined organic layers were washed with $\mathrm{HCl}(1 \mathrm{M}, 3 \mathrm{x})$. Extraction of the aqueous layer with $\mathrm{CH}_{2} \mathrm{Cl}_{2}(3 \mathrm{x})$, drying over $\mathrm{MgSO}_{4}$, filtration and concentration in vacuo yielded a the crude product as off-white solid. This crude product was suspended in $\mathrm{PrOH}$ and stirred over night. Filtration yielded $\mathbf{S} 11$ as a colorless solid $(3.76 \mathrm{~g}, 16.17 \mathrm{mmol}, 44 \%)$.

${ }^{1} \mathrm{H}-\mathrm{NMR}\left(600 \mathrm{MHz}, \mathrm{CDCl}_{3}\right): \delta=9.91\left(\mathrm{~s}, 2 \mathrm{H}, \mathrm{NH}_{2}\right), 7.27\left(\mathrm{~d},{ }^{3} \mathrm{~J}_{\mathrm{HH}}=8.4 \mathrm{~Hz}, 2 \mathrm{H}, \mathrm{H} 8\right), 7.17\left(\mathrm{~d},{ }^{3} \mathrm{~J}_{\mathrm{HH}}=\right.$ $8.4 \mathrm{~Hz}, 2 \mathrm{H}, \mathrm{H} 7), 6.14-6.06(\mathrm{~m}, 1 \mathrm{H}, \mathrm{H} 2), 5.50\left(\mathrm{~d},{ }^{3} \mathrm{~J}_{\mathrm{HH}}=17.1 \mathrm{~Hz}, 1 \mathrm{H}, \mathrm{H} 1-\mathrm{cis}\right), 5.46\left(\mathrm{~d},{ }^{3} \mathrm{~J}_{\mathrm{HH}}=\right.$ $10.2 \mathrm{~Hz}, 1 \mathrm{H}, \mathrm{H} 1$-trans), $3.63\left(\mathrm{q},{ }^{3} \mathrm{~J}_{\mathrm{HH}}=4.8 \mathrm{~Hz}, 2 \mathrm{H}, \mathrm{H} 3\right), 3.25-3.20(\mathrm{~m}, 2 \mathrm{H}, \mathrm{H} 5), 3.15-3.08(\mathrm{~m}$, $3 \mathrm{H}, \mathrm{H} 4)$ ppm; ${ }^{13} \mathrm{C}\left\{{ }^{1} \mathrm{H}\right\}-N M R\left(151 \mathrm{MHz}, \mathrm{CDCl}_{3}\right): \delta=135.0$ (C6), 133.2 (C9), 130.2 (C7), 129.2 (C8), 127.7 (C2), 124.5 (C1), 49.8 (C3), 47.6 (C4), 31.7 (C5) ppm; IR (ATR): $\tilde{v}=2940$ (w), 2761 (w), $2705(w), 2642(w), 2427(w), 1894(w), 1640(w), 1598(w), 1493(m), 1475(w), 1439(m)$, 1424 (w), $1412(\mathrm{w}), 1385(\mathrm{w}), 1341(\mathrm{w}), 1286(\mathrm{w}), 1219(\mathrm{~m}), 1164(\mathrm{~m}), 1125(\mathrm{w}), 1112(\mathrm{w}), 1092$ $(\mathrm{m}), 1036(\mathrm{~m}), 1011(\mathrm{~m}), 992(\mathrm{~m}), 964(\mathrm{w}), 942(\mathrm{~s}), 869(\mathrm{w}), 835(\mathrm{~m}), 823(\mathrm{~m}), 806(\mathrm{~s}), 782(\mathrm{w})$, 712 (w), 685 (m), 672 (s) cm ${ }^{-1}$; HR-ESI-MS: $\mathrm{m} / \mathrm{z}$ : [M-Cl] $]^{+}$calcd. for $\mathrm{C}_{11} \mathrm{H}_{15} \mathrm{NCl}^{+}$: 196.0888; found: 196.0902; m.p.: $172-173^{\circ} \mathrm{C}$ (decomposition). The analytical data are in agreement with the litarature. ${ }^{[20]}$

rac-8-Chloro-1-methyl-2,3,4,5-tetrahydro-1H-benzo[d]azepine $(R, S)$-Lorcaserin (rac-24)

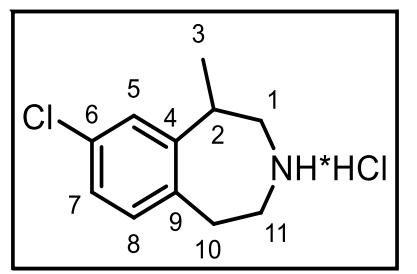

hydrochloric acid;

S11 (1.00 g, $4.30 \mathrm{mmol}, 1.0$ eq.) and $\mathrm{AlCl}_{3}(1.00 \mathrm{~g}, 7.54 \mathrm{mmol}$, 1.75 eq.) were combined in a dry Schlenk pressure tube and stirred at $125^{\circ} \mathrm{C}$ for $4 \mathrm{~h}$. The hot, molten reaction mixture was poured into a $-5^{\circ} \mathrm{C}$ cooled brine solution and the reaction tube was rinsed with $\mathrm{H}_{2} \mathrm{O}$ and $\mathrm{CH}_{2} \mathrm{Cl}_{2}$ (cave: $\mathrm{HCl}$ gas evolution). This mixture was stirred overnight before being filtered over Celite ${ }^{\circledR}$. The layers were separated and the aqueous layer was extracted with $\mathrm{CH}_{2} \mathrm{Cl}_{2}(3 \mathrm{x})$. The combined organic layers were dried over MgSO4, filtered and concentrated in vacuo, The residue was suspended in acetone and stirred for $30 \mathrm{~min}$ at room temperature. The solid was filtrated and washed with acetone to yield rac-24 as a colorless solid (426 mg, $1.84 \mathrm{mmol}, 43 \%$ ).

\section{(R)-8-Chloro-1-methyl-2,3,4,5-tetrahydro-1H-benzo[d]azepine, $(R)$-Lorcaserin $((R)$-24)}

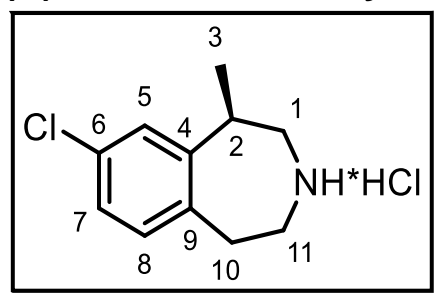

Following an adapted literature procedure, ${ }^{[7]}(\boldsymbol{R})-\mathrm{S} 10$ (20 mg, $0.068 \mathrm{mmol}$, 1.0 eq.) was dissolved in dry $\mathrm{CH}_{2} \mathrm{Cl}_{2}(662 \mu \mathrm{L})$ in a flame dried Schlenk pressure tube under an argon atmosphere and $\mathrm{HSiEt}_{3}(22 \mu \mathrm{L}, 0.136 \mathrm{mmol}, 2 \mathrm{eq}$.) and trifluoroacetic acid $(49 \mu \mathrm{L}$, $7 \% \mathrm{v} / \mathrm{v}$ ) were added. The reaction mixture was stirred at room temperature for $2 \mathrm{~h}$ before a sat. aq. solution of $\mathrm{NaHCO}_{3}$ was added 
slowly until gas evolution stopped. The $\mathrm{pH}$ was adjusted to $\mathrm{pH} 10$ by addition of solid $\mathrm{NaOH}$ and the resulting mixture was vigurously stirred for $20 \mathrm{~min}$. The mixture was extracted with EtOAc $(3 \mathrm{x})$ and the combined organic layers were washed with $\mathrm{H}_{2} \mathrm{O}$, brine, dired over $\mathrm{Na}_{2} \mathrm{SO}_{4}$, filtered and concentrated in vacuo. The crude product was dissolved in $\mathrm{HCl}(4 \mathrm{M}$ in 1,4-dioxane, $0.5 \mathrm{~mL}$, $2 \mathrm{mmol}, 30$ eq.) at $0{ }^{\circ} \mathrm{C}$ and stirred for $30 \mathrm{~min}$ at room temperature. The solvent was removed in vacuo and the remaining solid was washed with $\mathrm{Et}_{2} \mathrm{O}$ to yield $(\boldsymbol{R})-\mathbf{2 4}$ as colorless solid $(1.2 \mathrm{mg}, 0.005 \mathrm{mmol}, 8 \%)$. The enantiomeric ratio was found to be $81: 19$ by chiral HPLC (Chiralpac ${ }^{\circledR} \mathrm{IA}, n$-hexane:EtOH:MeOH:diethylamine 95:2.5:2.5:0.1, $1.0 \mathrm{~mL} / \mathrm{min}, \lambda=220 \mathrm{~nm}$, $t_{R}$ (major): 7.056 min, $t_{R}$ (minor): $8.436 \mathrm{~min}$ ).

$\mathbf{R}_{\boldsymbol{f}}=\left(\right.$ EtOAc:cyclohexane); ${ }^{1} \mathrm{H}-\mathrm{NMR}\left(600 \mathrm{MHz}, \mathrm{CDCl}_{3}\right): \delta=10.21\left(\mathrm{br} \mathrm{s}, \mathrm{NH}_{2}\right), 9.93\left(\mathrm{br} \mathrm{s}, \mathrm{NH}_{2}\right)$, $7.20\left(\mathrm{~d},{ }^{4} \mathrm{~J}_{\mathrm{HH}}=2.1 \mathrm{~Hz}, 1 \mathrm{H}, \mathrm{H} 5\right), 7.18\left(\mathrm{dd},{ }^{3} \mathrm{JHH}_{\mathrm{HH}}=8.1 \mathrm{~Hz},{ }^{4} \mathrm{~J}_{\mathrm{HH}}=2.1 \mathrm{~Hz}, 1 \mathrm{H}, \mathrm{H} 7\right), 7.09\left(\mathrm{~d},{ }^{3} \mathrm{~J}_{\mathrm{HH}}=\right.$ $8.1 \mathrm{~Hz}, 1 \mathrm{H}, \mathrm{H} 8), 3.66-3.40$ (m, 4H, H2, H1, H10, H11), $3.10-2.82$ (m, 3H, H1', H10', H11'), $1.50\left(\mathrm{~d},{ }^{3} \mathrm{~J}_{\mathrm{HH}}=7.1 \mathrm{~Hz}, \mathrm{H} 3\right) \mathrm{ppm} ;{ }^{13} \mathrm{C}\left\{{ }^{1} \mathrm{H}\right\}-\mathrm{NMR}\left(126 \mathrm{MHz}, \mathrm{CDCl}_{3}\right): \delta=144.5(\mathrm{C} 4), 137.0(\mathrm{C} 9)$, 133.6 (C6), 131.3 (C8), 127.4 (C7), 126.5 (C5), 51.6 (C1), 45.7 (C11), 35.0 (C2), 32.2 (C10), 18.1 (C3) ppm; IR (ATR): $\tilde{v}=2979$ (w), 2685 (s), 2644 (s), 2600 (m), 2499 (m), 2430 (w), 1580 (s), $1485(\mathrm{~m}), 1427(\mathrm{w}), 1403(\mathrm{~m}), 1390(\mathrm{~m}), 1377(\mathrm{w}), 1331(\mathrm{w}), 1316(\mathrm{w}), 1287(\mathrm{w}), 1263(\mathrm{w})$, 1231 (w), $1185(w), 1172(w), 1127(w), 1105$ (w), $1081(w), 1059(w), 1024(w), 1008$ (w), 932 (s), $876(\mathrm{~m}), 831(\mathrm{~m}), 747(\mathrm{w}), 681(\mathrm{w}) \mathrm{cm}^{-1}$; HR-ESI-MS: $\mathrm{m} / \mathrm{z}$ : $[\mathrm{M}+\mathrm{H}]^{+}$calcd. for $\mathrm{C}_{11} \mathrm{H}_{15} \mathrm{NCl}^{+}$: 196.0888; found: 196.0896 ; m.p.: $207-209^{\circ} \mathrm{C}$ (decomposition). The analytical data are in agreement with the literature..$^{[7,21]}$

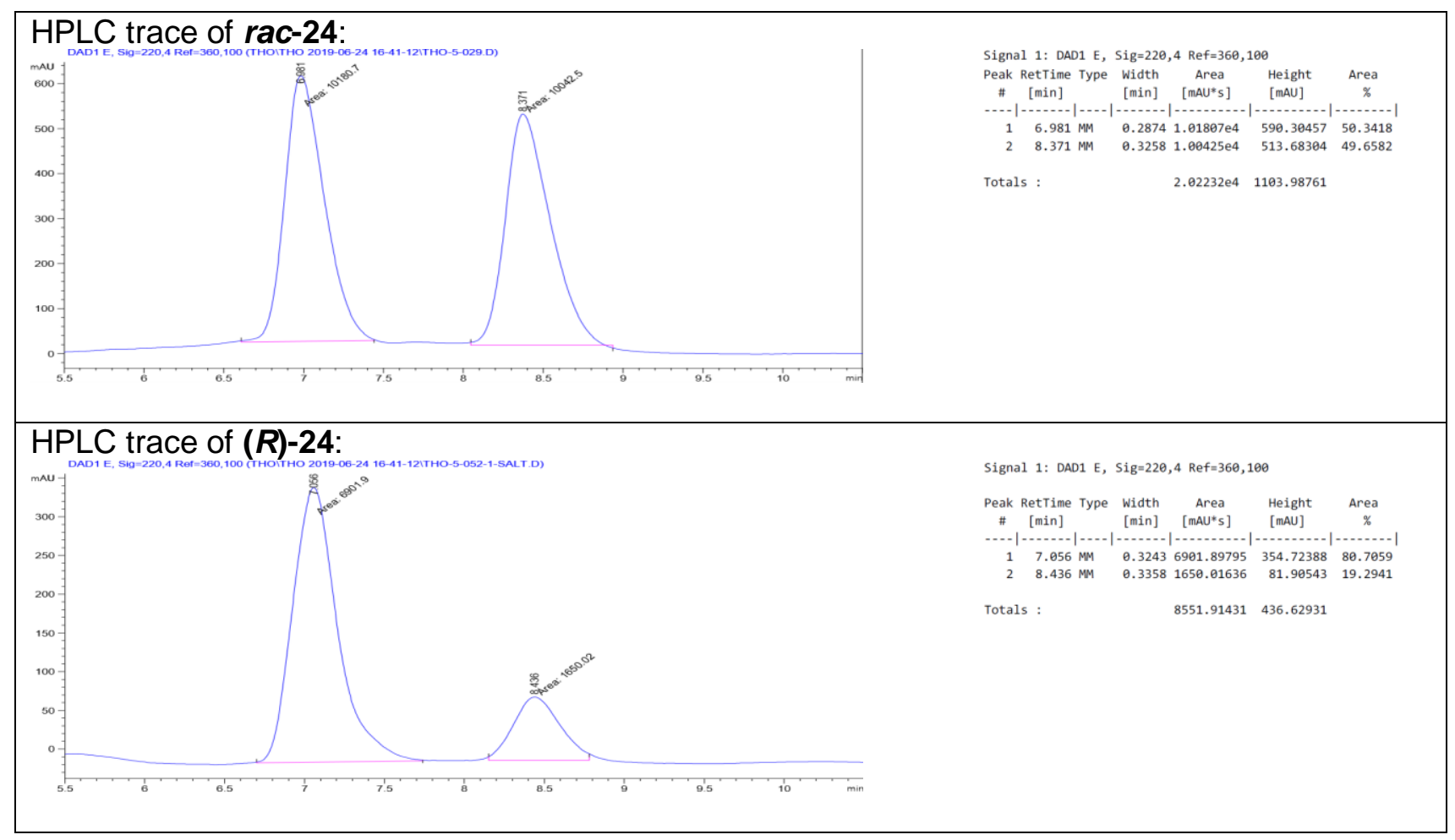

Figure 18: HPLC traces of 24; top: rac-24; bottom: $(\boldsymbol{R}) \mathbf{- 2 4}$. 


\section{References}

[1] Martin, Nolwenn J. A.; Ozores, L.; List, B. J. Am. Chem. Soc. 2007, 129, 8976-8977

[2] Gärtner, D.; Stein, A. L.; Gruppe, S.; Arp, J.; Jacobi von Wagelin, A. Angew. Chem. Int. Ed. 2015, 54, 10545-10549.

[3] Wu, X.; Riedel, J.; Dong, V. M. Angew. Chem. Int. Ed. 2017, 56, 11589-11593.

${ }^{[4]}$ Schneider, J. F.; Lauber, M. B.; Muhr, V.; Kratzer, D.; Paradies, J. Org. Biomol. Chem. 2011, 9, 43234327.

[5] Li, S.; Huang, K.; Cao, B.; Zhang, J.; Wu, W.; Zhang, X. Angew. Chem. Int. Ed. 2012, 51, 8573-8576.

[6] Sim, J. H.; Song, C. E. Angew. Chem. Int. Ed. 2017, 34, 1861-1865.

[7] Gini, A.; Bamberger, J.; Luis-Barrera, J.; Zurro, M.; Mas-Ballesté, R.; Alemán, J.; García Mancheño, O. Adv. Synth. Catal. 2016, 358, 4049-4056.

${ }^{[8]}$ Li, S.; Xu, S.; Cui,, S.; Wu, F.; Zhang, Y.; Su, M.; Gong, Y.; Quu, S.; Jiao, Q.; Qin, C.; Shan, J.; Zhang, M.; Wang, J.; Yin, Q.; Xu, M.; Liu, X.; Wang, R.; Zhu, L.; Li, J.; Xu, Y.; Jiang, H.; Zhao, Z.; Li, J.; Li, H. J. Med. Chem. 2016, 59, 6772-6790.

[9] Zhang, M.; Hu, P.; Zhou, J.; Wu, G.; Huang, S.; Su, W. Org. Lett. 2013, 15, 1718-1721.

[10] Toogood, H. S.; Fryszkowska, A.; Hare, V.; Fisher, K.; Roujeinikova, A.; Leys, D.; Gardiner, J.;

Stephens, G.; Scrutton, N. Adv. Synth. Catal. 2008, 350, 2789-2803.

[11] Fryszkowska, A.; Fisher, K.; Gardiner, J. M.; Stephens, G. M. J. Org. Chem. 2008, 73, 4295-5298.

[12] Vuagnoux-d'Augustin, M.; Alexakis, A. Eur. J. Org. Chem. 2007, 35, 5852-5860.

[13] Liu, M.; Kong, D.; Li, M.; Zi, G.; Hou, G. Adv. Synth. Catal. 2015, 357, 3875-3879.

[14] Mampreian, D. M.; Hoveyda, A. H. Org. Lett. 2004, 6, 2829-2832.

[15] Soltani, O.; Ariger, M. A.; Carreira, E. M. Org. Lett. 2009, 11, 4196-4198.

[16] Christoffers, J.; Mann, A. Chem. Eur. J. 2001, 7, 1014-1027.

[17] Massolo, E.; Benaglia, M.; Orlandi, M.; Rossi, S.; Celentano, G. C. Chem. Eur. J. 2015, 21, 35893595.

[18] Klausen, R. S.; Kennedy, C. R.; Hyde, A. M.; Jacobsen, E. N. J. Am. Chem. Soc. 2017, 139, 1229912309.

[19] Vogler, T.; Studer, A. Adv. Synth. Catal. 2008, 350, 1963-1967.

[20] Cluzeau, J.; Stavber, G. Bioorg. Med. Chem. 2018, 26, 977-983.

[21] Wani, D. V.; Rane, V. P.; Mokale, S. N. Chirality 2018, 30, 284-292. 


\section{UV/Vis Absorption spectra}

Spectra was recorded on an Agilent Cary60 in acetonitrile at ambient temperature, with a pathlength of $1 \mathrm{~cm}$.

Molar absorption coeffients for $\boldsymbol{E}-\mathbf{1}$ and $\boldsymbol{Z}-1$ were calculated at $402 \mathrm{~nm}$ for concentrations of $1 \mathrm{mM}$ and $10 \mathrm{mM}$. E-1 $=85 \mathrm{dm}^{3} \mathrm{~mol}^{-1} \mathrm{~cm}^{-1} ; Z-1=42 \mathrm{dm}^{3} \mathrm{~mol}^{-1} \mathrm{~cm}^{-1}$.

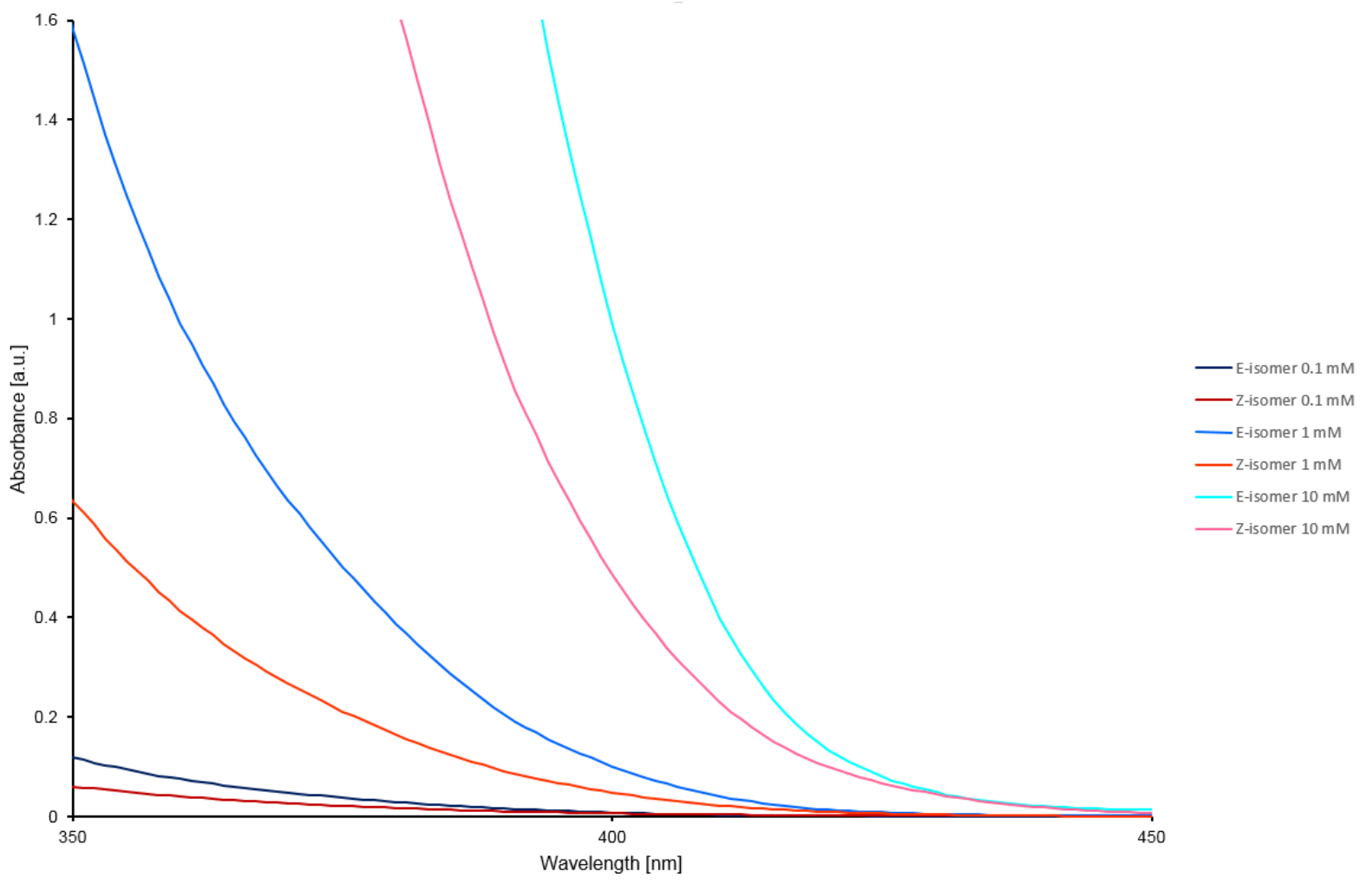

Figure 19: UV/Vis spectra (350-450 $\mathrm{nm})$ of $\boldsymbol{E}-\mathbf{1}$ and $\mathbf{Z}-\mathbf{1}$ at varying concentrations. 
NMR spectra of key compounds

(E)-1-(Cyclopropyl-2-nitrovinyl)benzene (E-10)

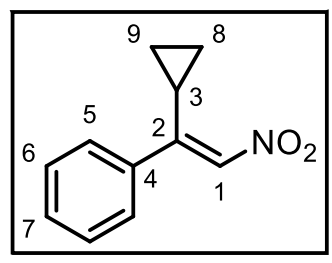

${ }^{1} \mathrm{H}-\mathrm{NMR}\left(500 \mathrm{MHz}, \mathrm{CDCl}_{3}\right)$

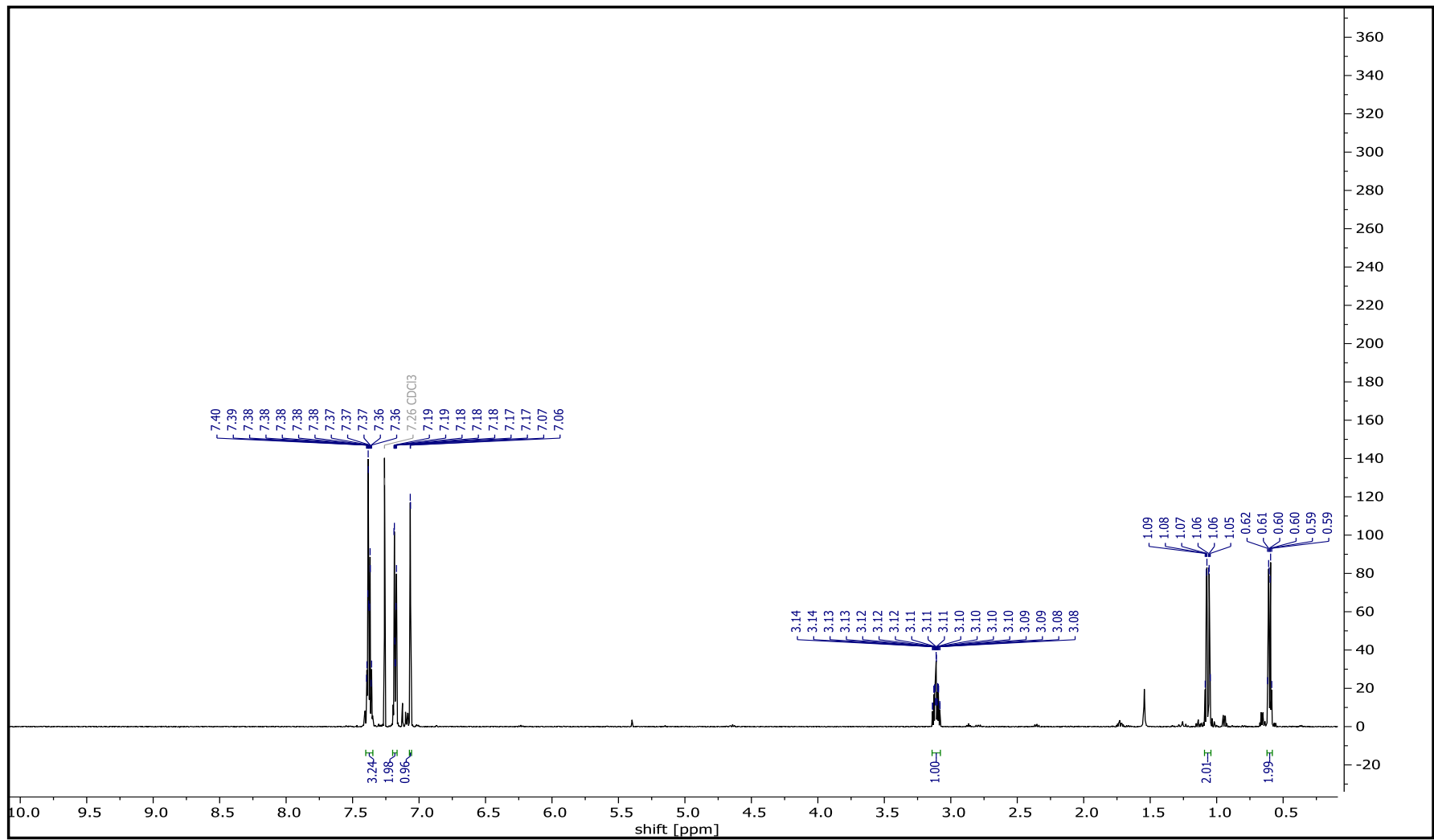

${ }^{13} \mathrm{C}-\mathrm{NMR}\left(126 \mathrm{MHz}^{\mathrm{CDCl}}{ }_{3}\right)$

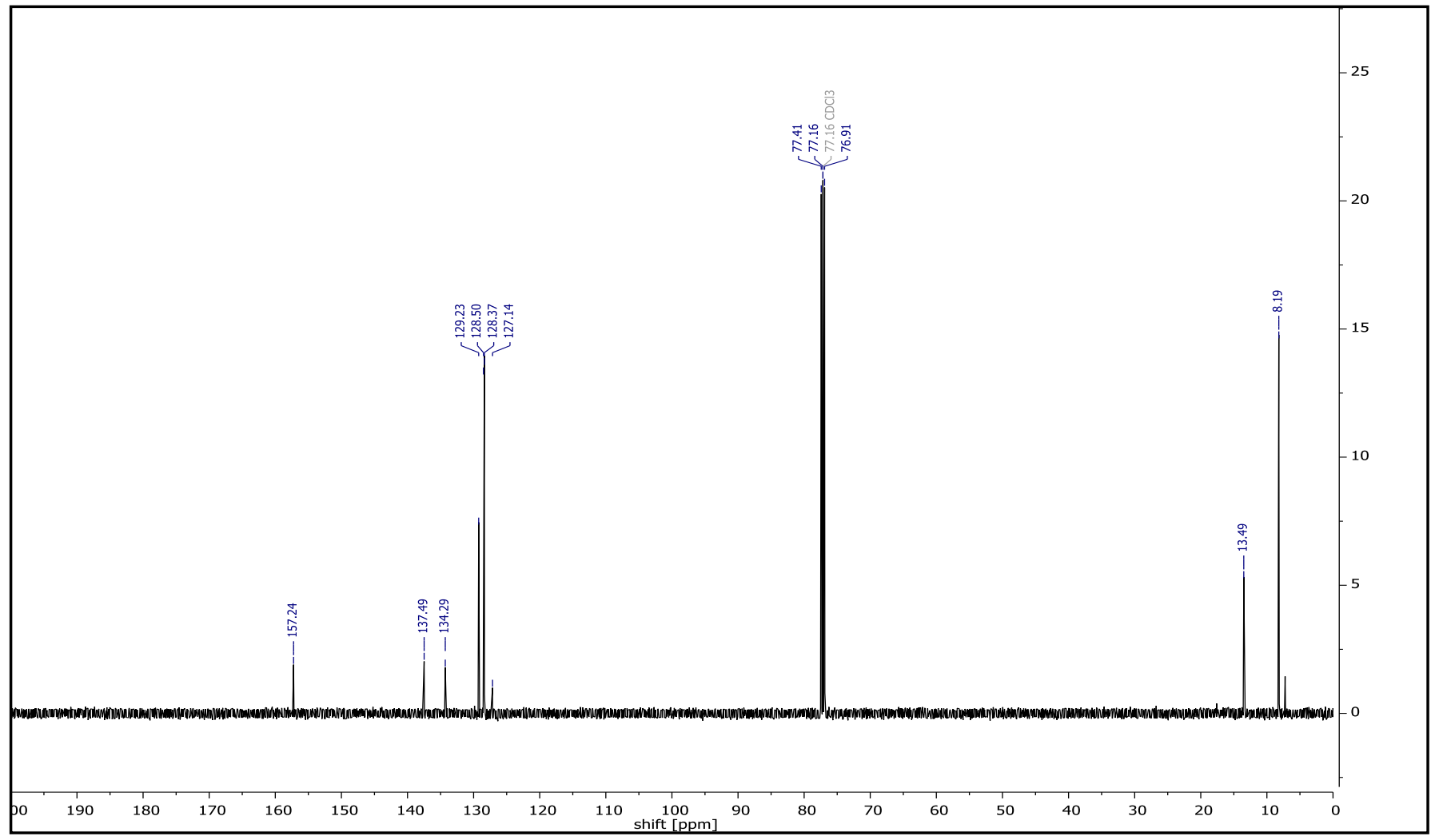


(Z)-(1-Nitroprop-1-en-2-yl)benzene (Z-1)

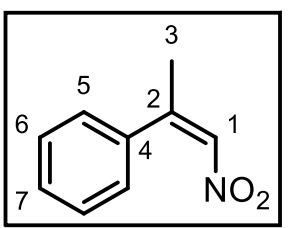

${ }^{1} \mathrm{H}-\mathrm{NMR}\left(600 \mathrm{MHz}, \mathrm{CDCl}_{3}\right)$

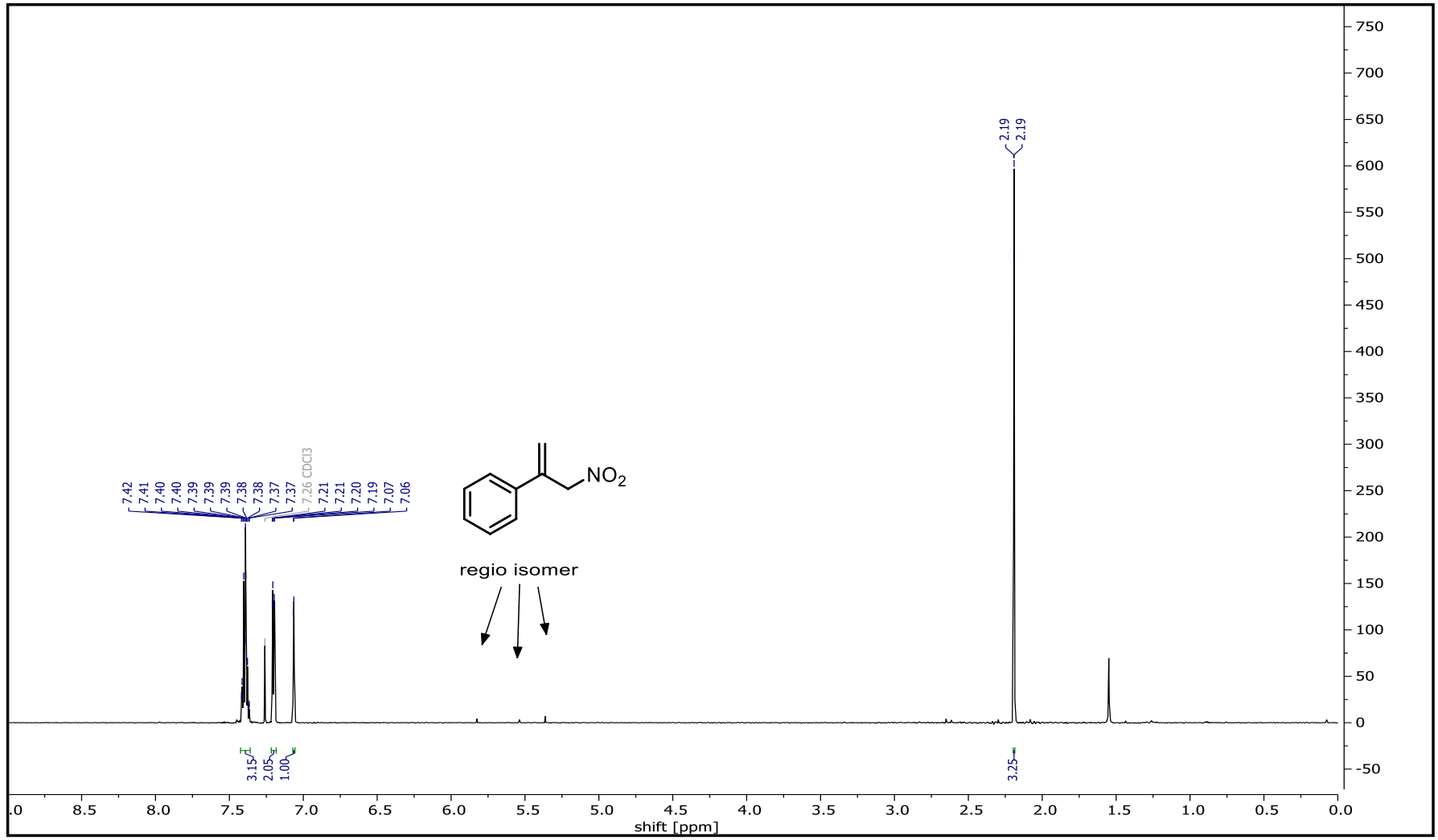

${ }^{13} \mathrm{C}-\mathrm{NMR}\left(151 \mathrm{MHz}, \mathrm{CDCl}_{3}\right)$

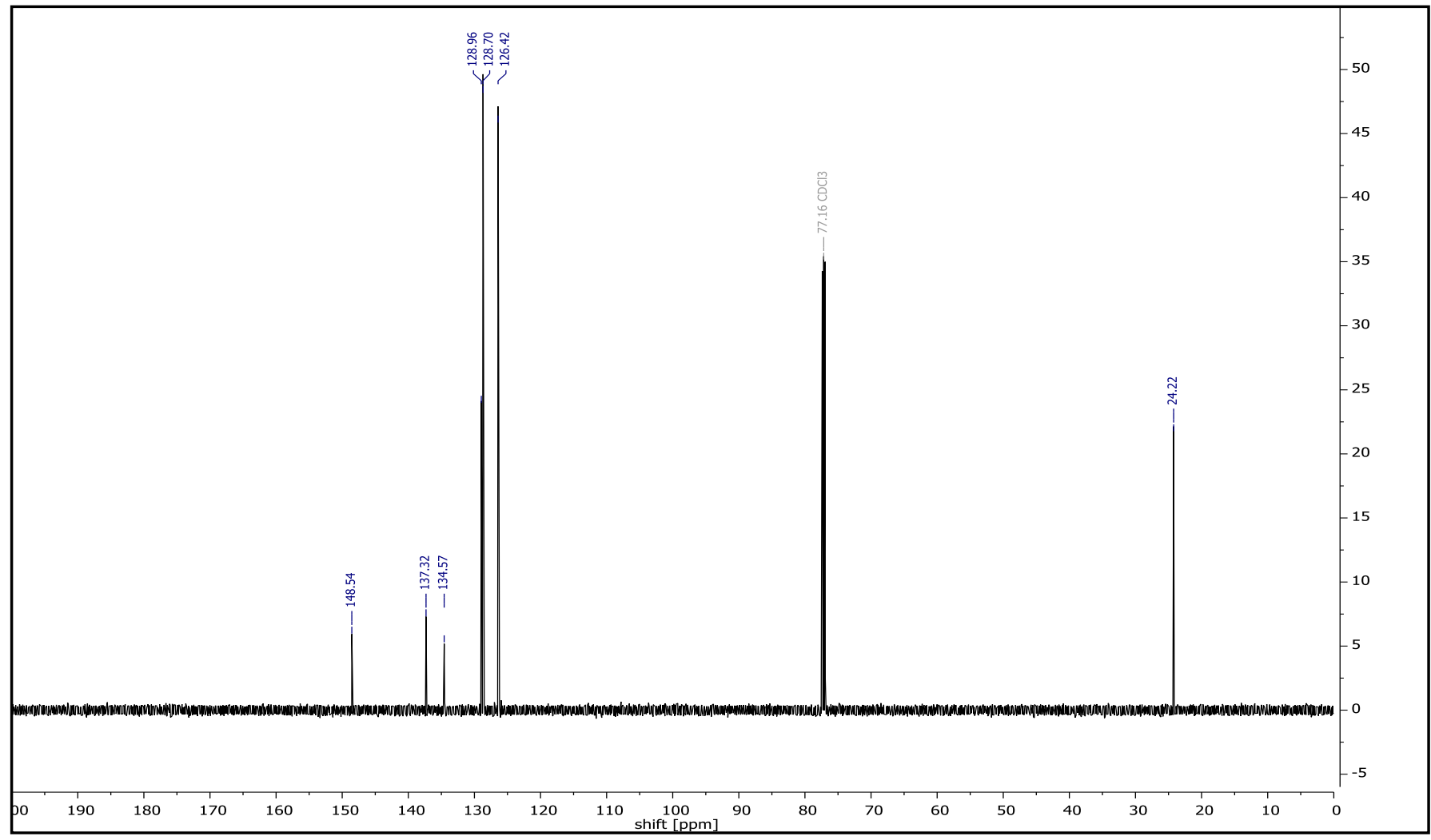


(Z)-1-Methyl-4-(1-nitroprop-1-en-2-yl)benzene (Z-2)

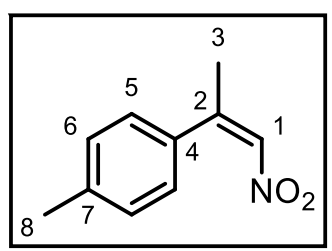

${ }^{1} \mathrm{H}-\mathrm{NMR}\left(600 \mathrm{MHz}, \mathrm{CDCl}_{3}\right)$

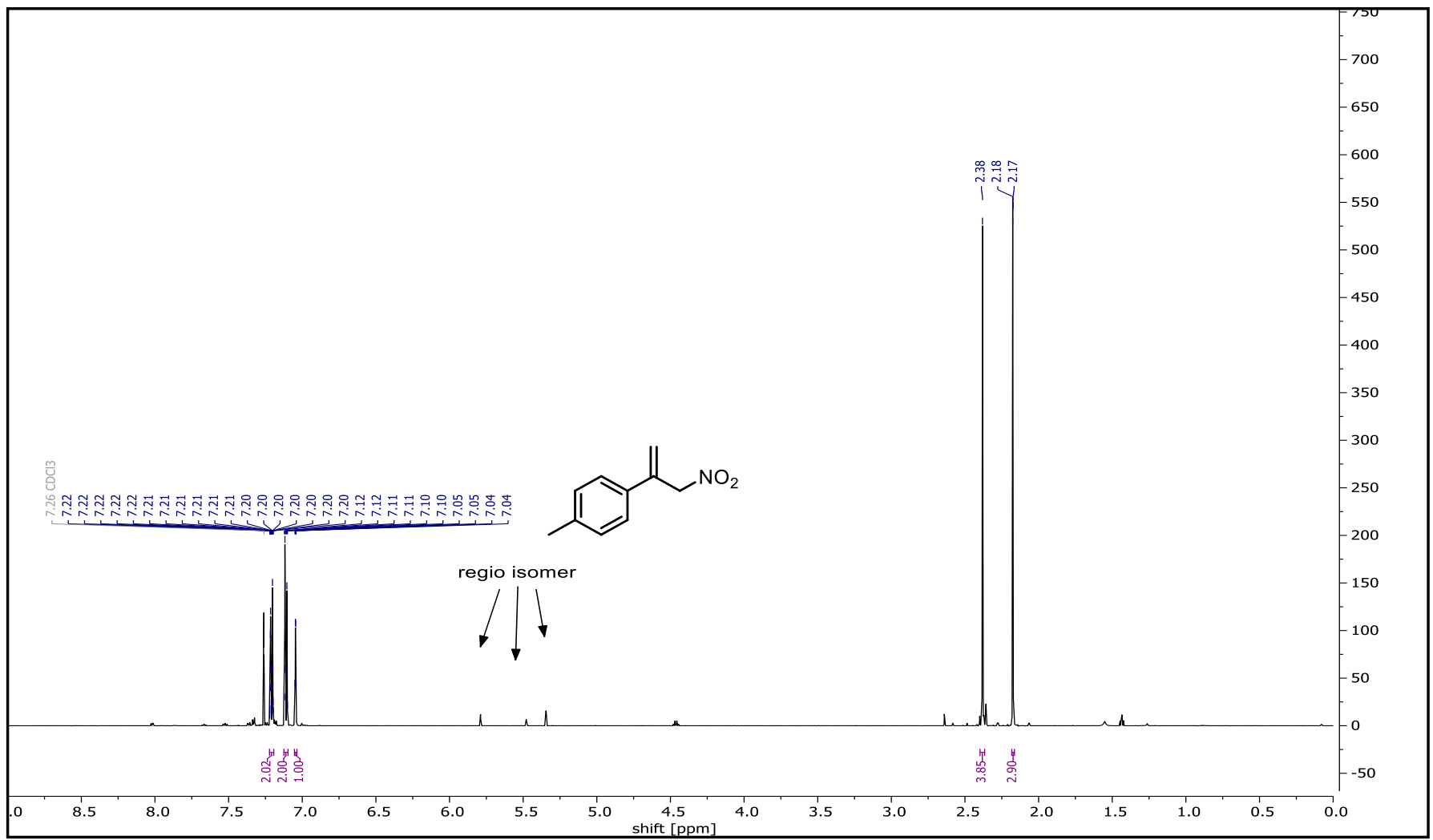

${ }^{13} \mathrm{C}-\mathrm{NMR}\left(151 \mathrm{MHz}, \mathrm{CDCl}_{3}\right)$

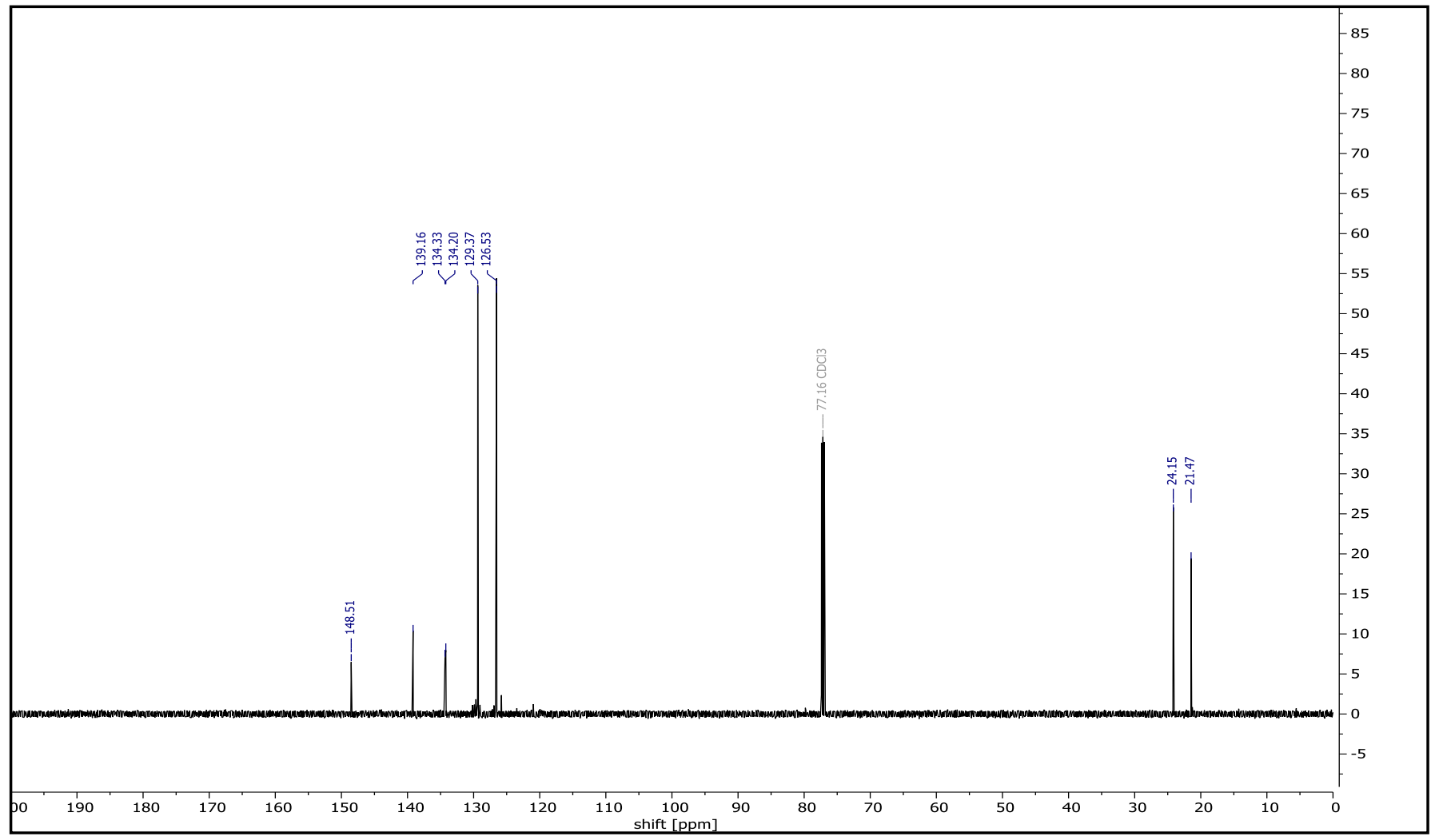


(Z)-1-Methoxy-4-(1-nitroprop-1-en-2-yl)benzene (Z-3)

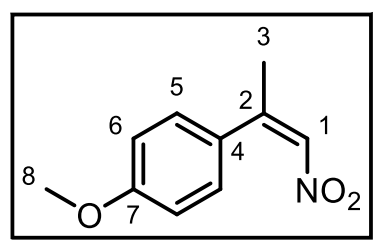

${ }^{1} \mathrm{H}-\mathrm{NMR}\left(500 \mathrm{MHz}, \mathrm{CDCl}_{3}\right)$

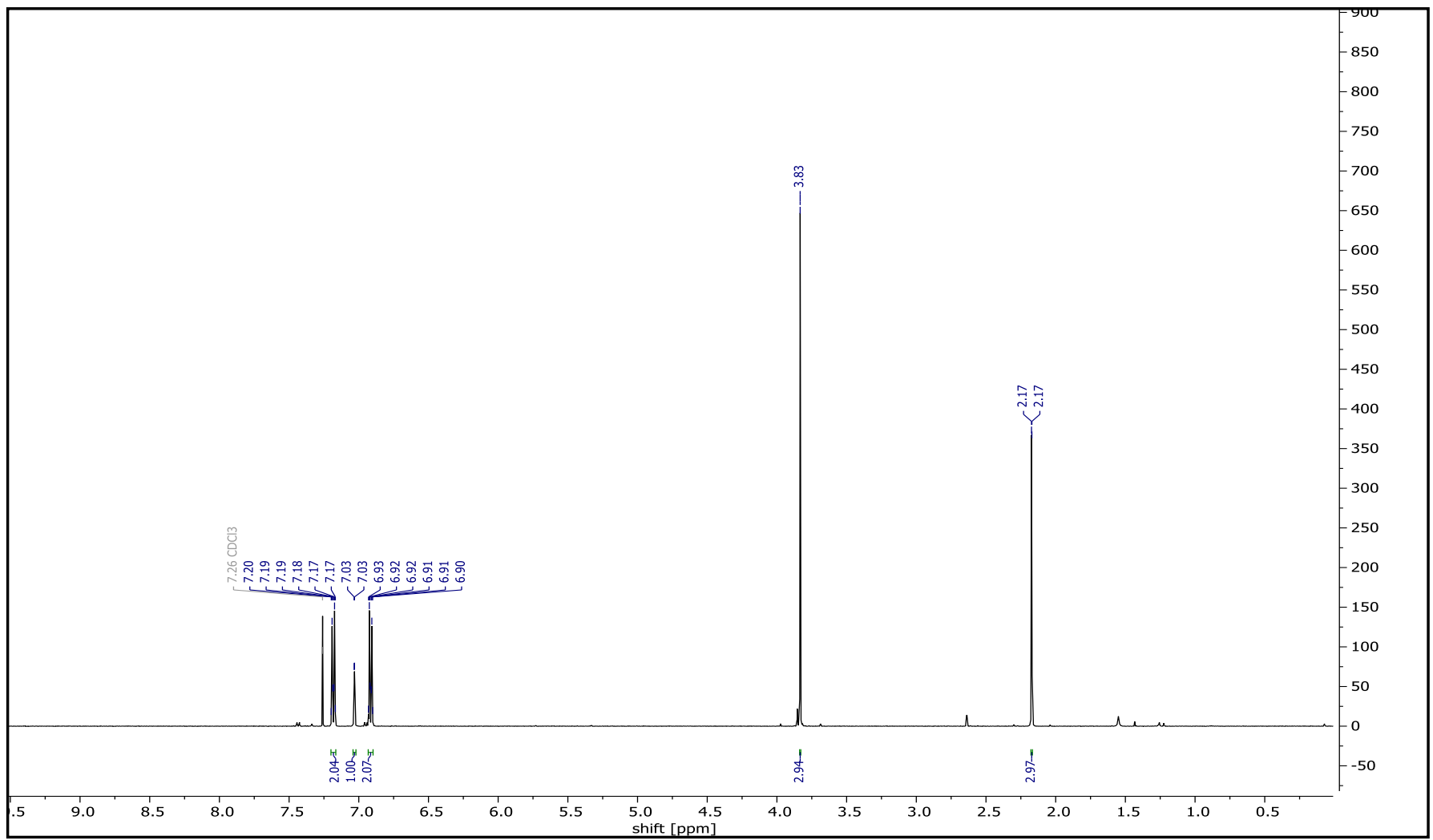

${ }^{13} \mathrm{C}-\mathrm{NMR}\left(126 \mathrm{MHz}_{\mathrm{CDCl}}\right)$

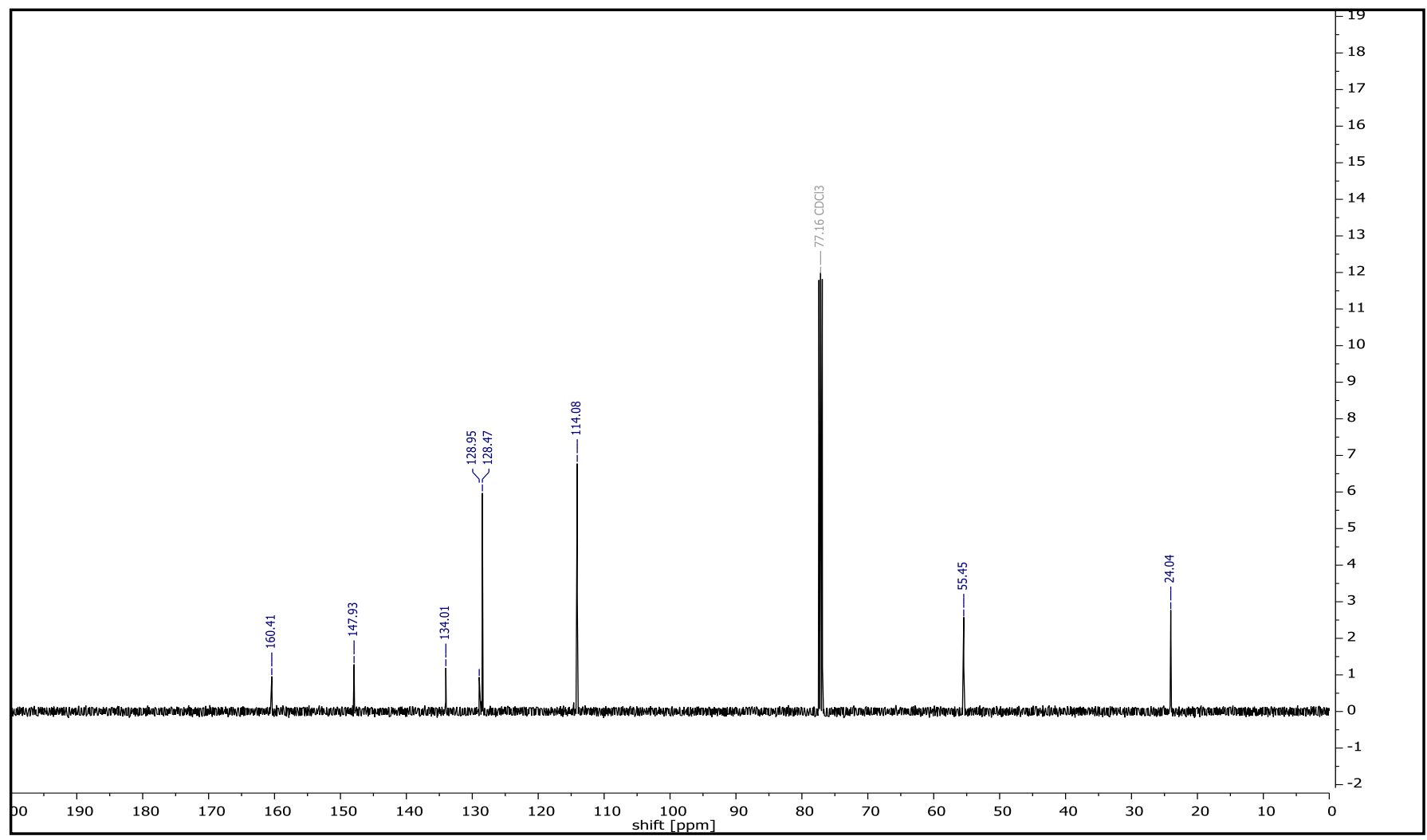


(Z)-1-Fluoro-4-(1-nitroprop-1-en-2-yl)benzene (Z-4)

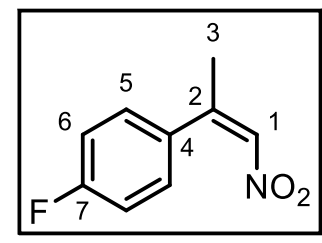

${ }^{1} \mathrm{H}-\mathrm{NMR}\left(500 \mathrm{MHz}, \mathrm{CDCl}_{3}\right)$

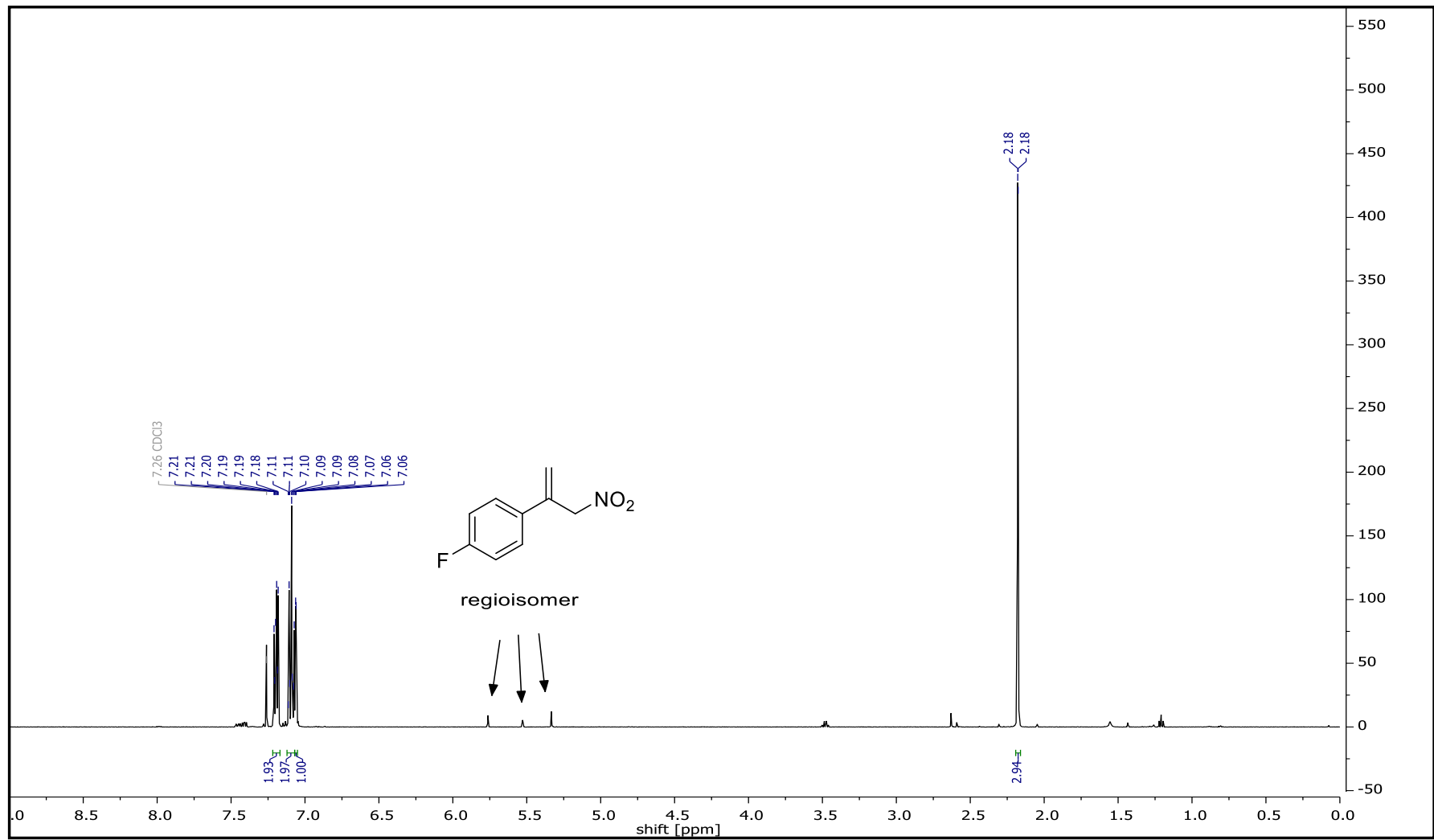

${ }^{13} \mathrm{C}-\mathrm{NMR}\left(126 \mathrm{MHz}_{\mathrm{CDCl}}\right)$

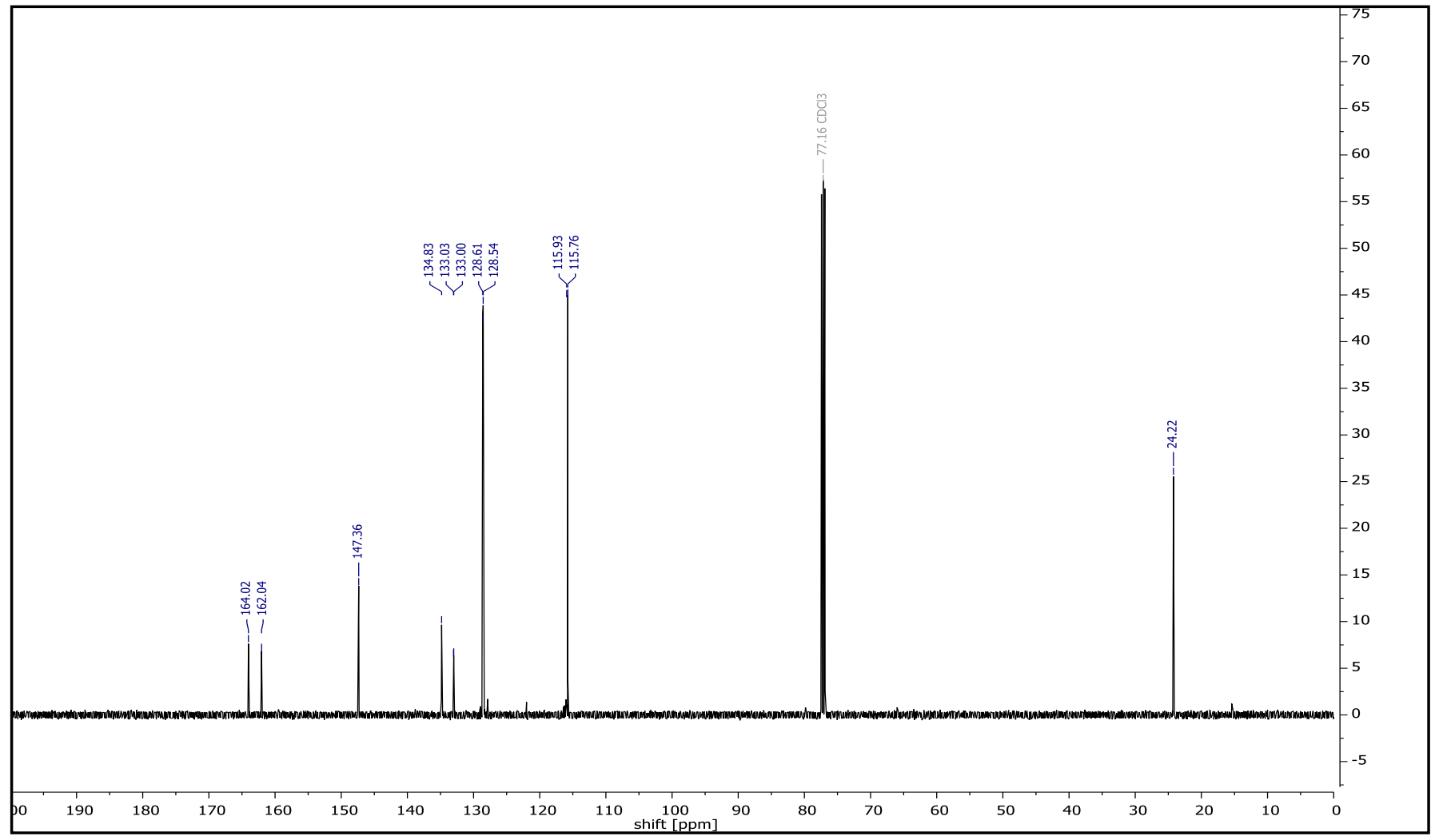


(Z)-1-Fluoro-4-(1-nitroprop-1-en-2-yl)benzene (Z-4)

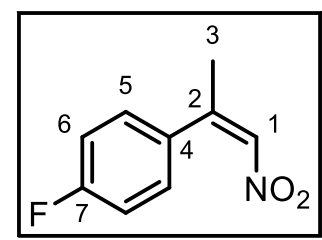

${ }^{19} \mathrm{~F}-\mathrm{NMR}\left(470 \mathrm{MHz}, \mathrm{CDCl}_{3}\right)$

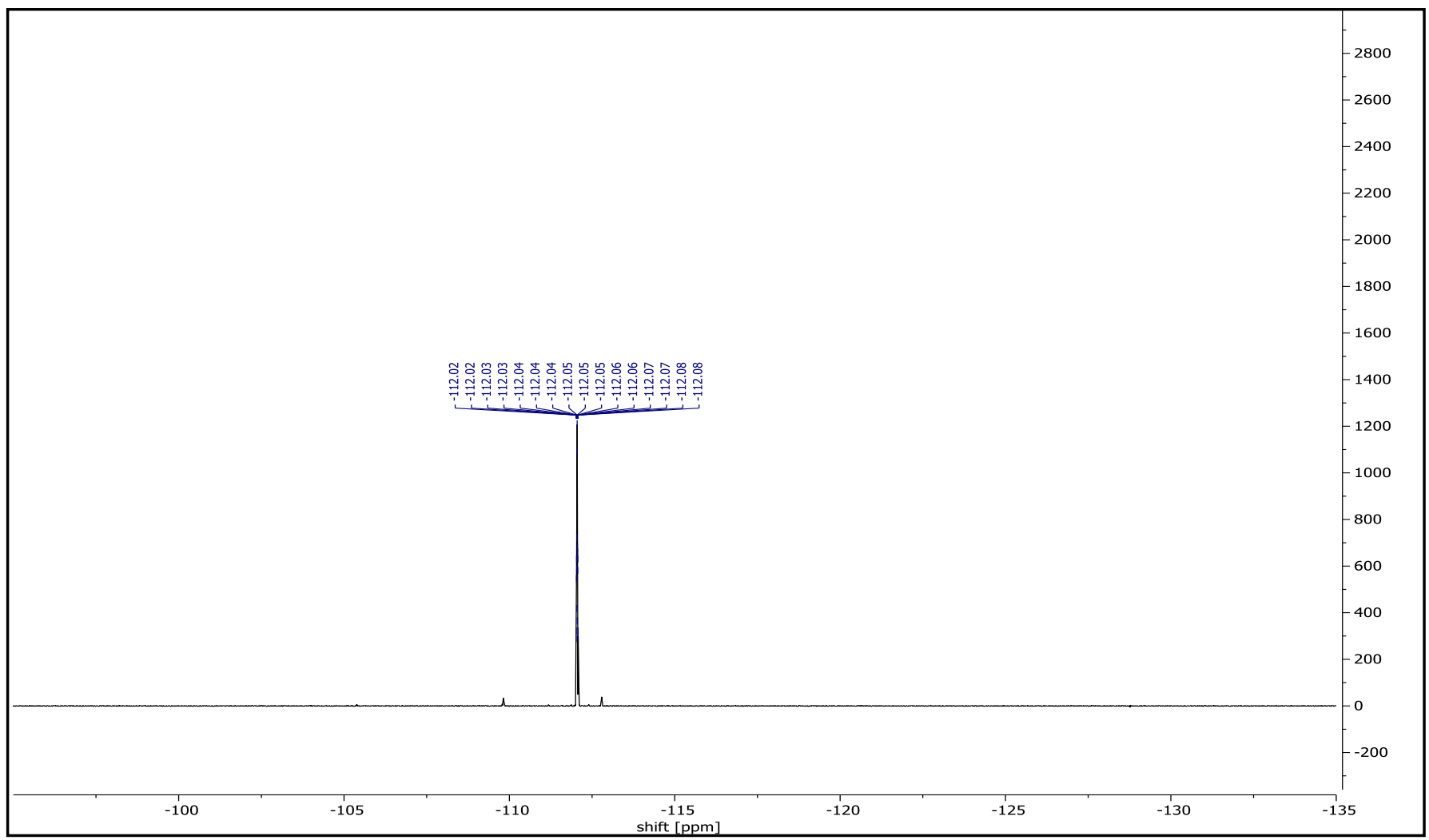


(Z)-1-(1-nitroprop-1-en-2-yl)-4-trifluoromethyl)benzene (Z-6)

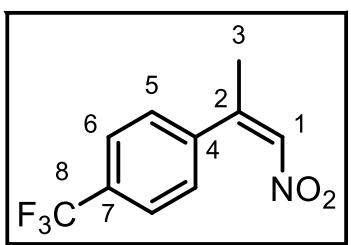

${ }^{1} \mathrm{H}-\mathrm{NMR}\left(500 \mathrm{MHz}, \mathrm{CDCl}_{3}\right)$

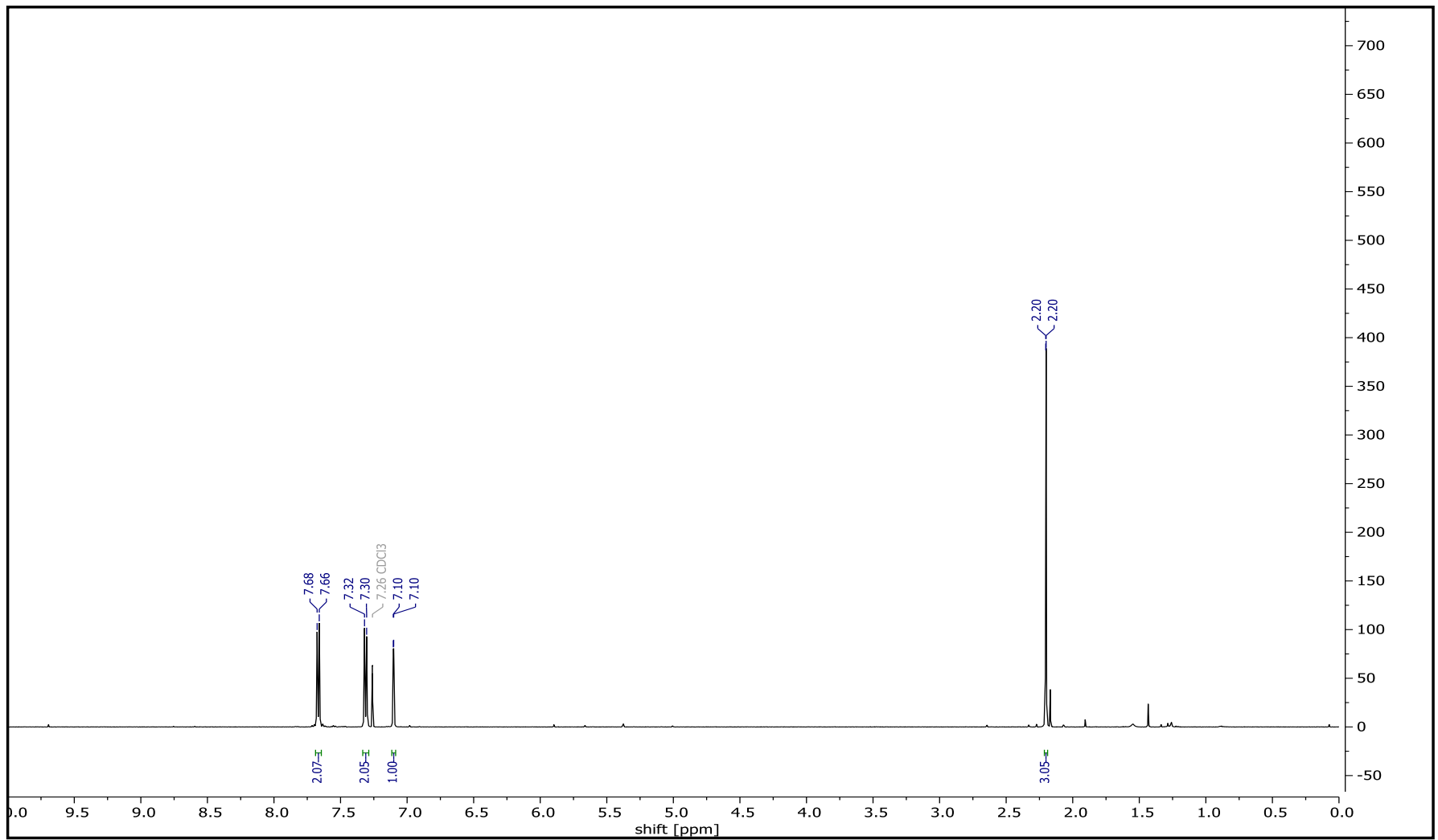

${ }^{13} \mathrm{C}-\mathrm{NMR}\left(126 \mathrm{MHz} \mathrm{CDCl}_{3}\right)$

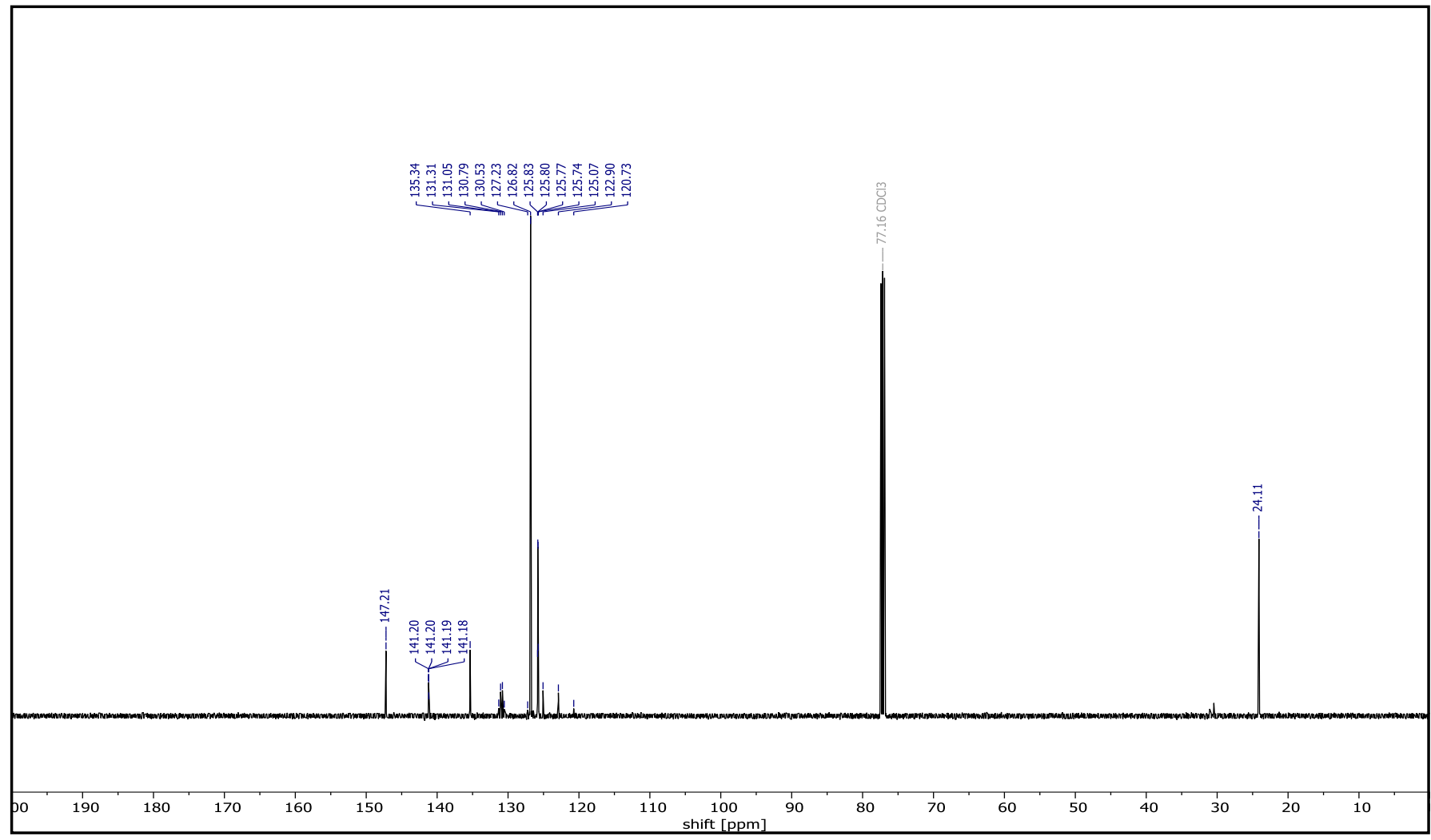


(Z)-1-(1-nitroprop-1-en-2-yl)-4-trifluoromethyl)benzene (Z-6)

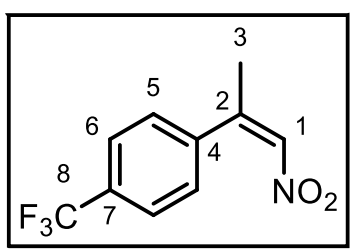

${ }^{19} \mathrm{~F}-\mathrm{NMR}\left(470 \mathrm{MHz}, \mathrm{CDCl}_{3}\right)$

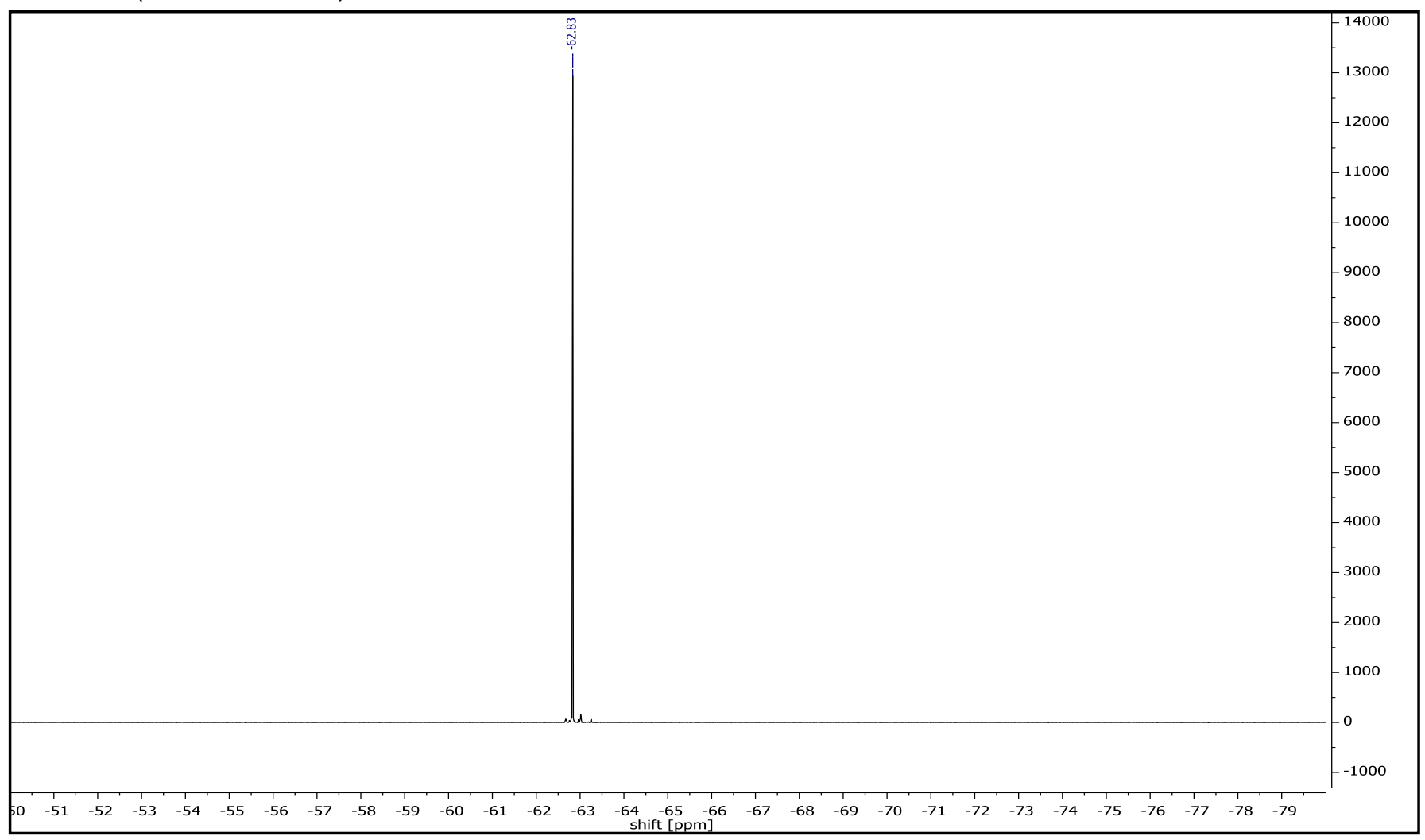


(Z)-1-Methyl-2-(1-nitroprop-1-en-2-yl)benzene (Z-7)

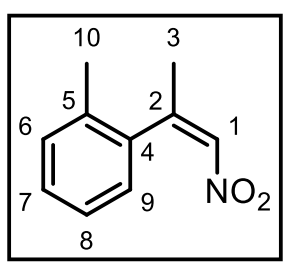

${ }^{1} \mathrm{H}-\mathrm{NMR}\left(500 \mathrm{MHz}^{\mathrm{CDCl}}{ }_{3}\right)$

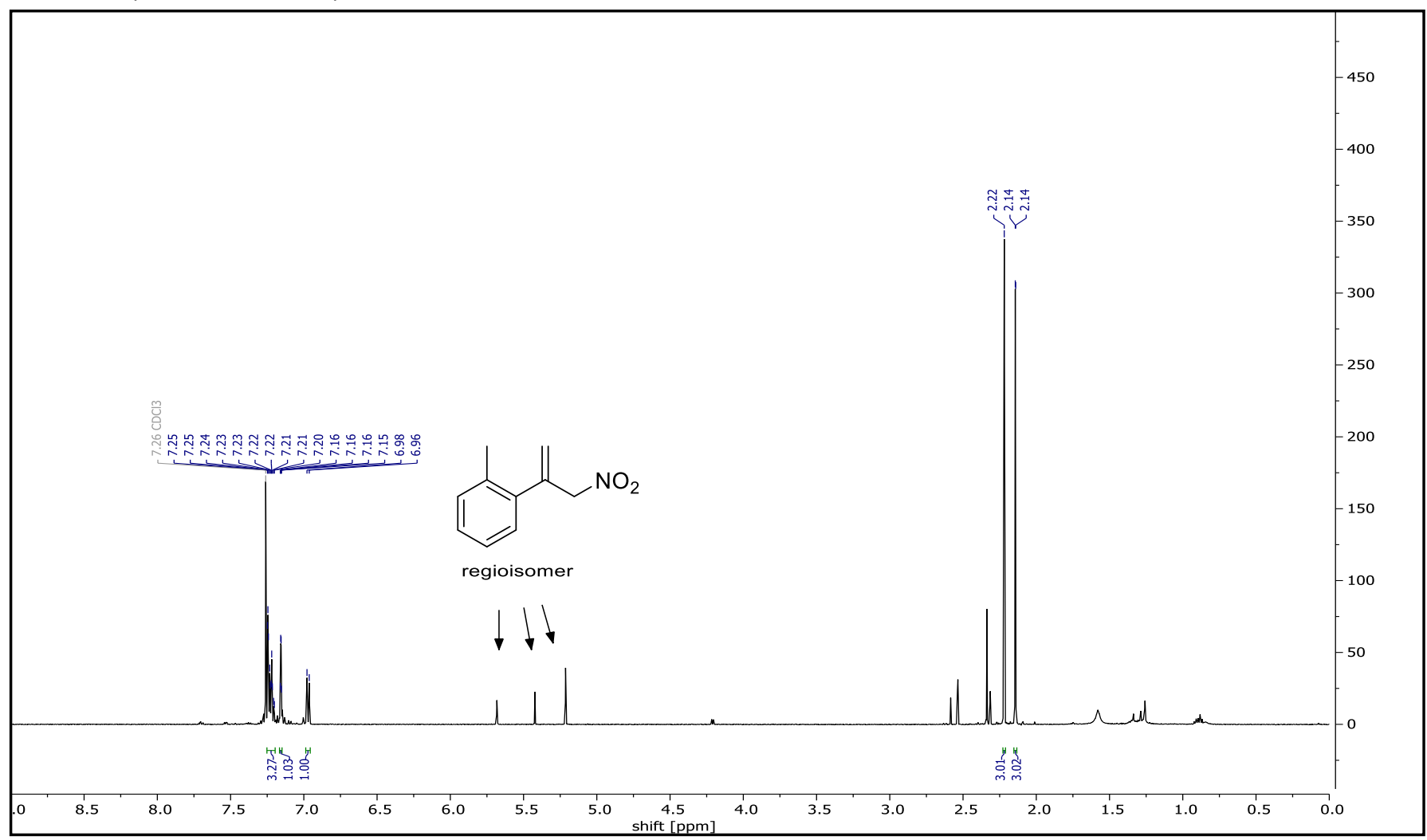

${ }^{13} \mathrm{C}-\mathrm{NMR}\left(126 \mathrm{MHz} \mathrm{CDCl}_{3}\right)$

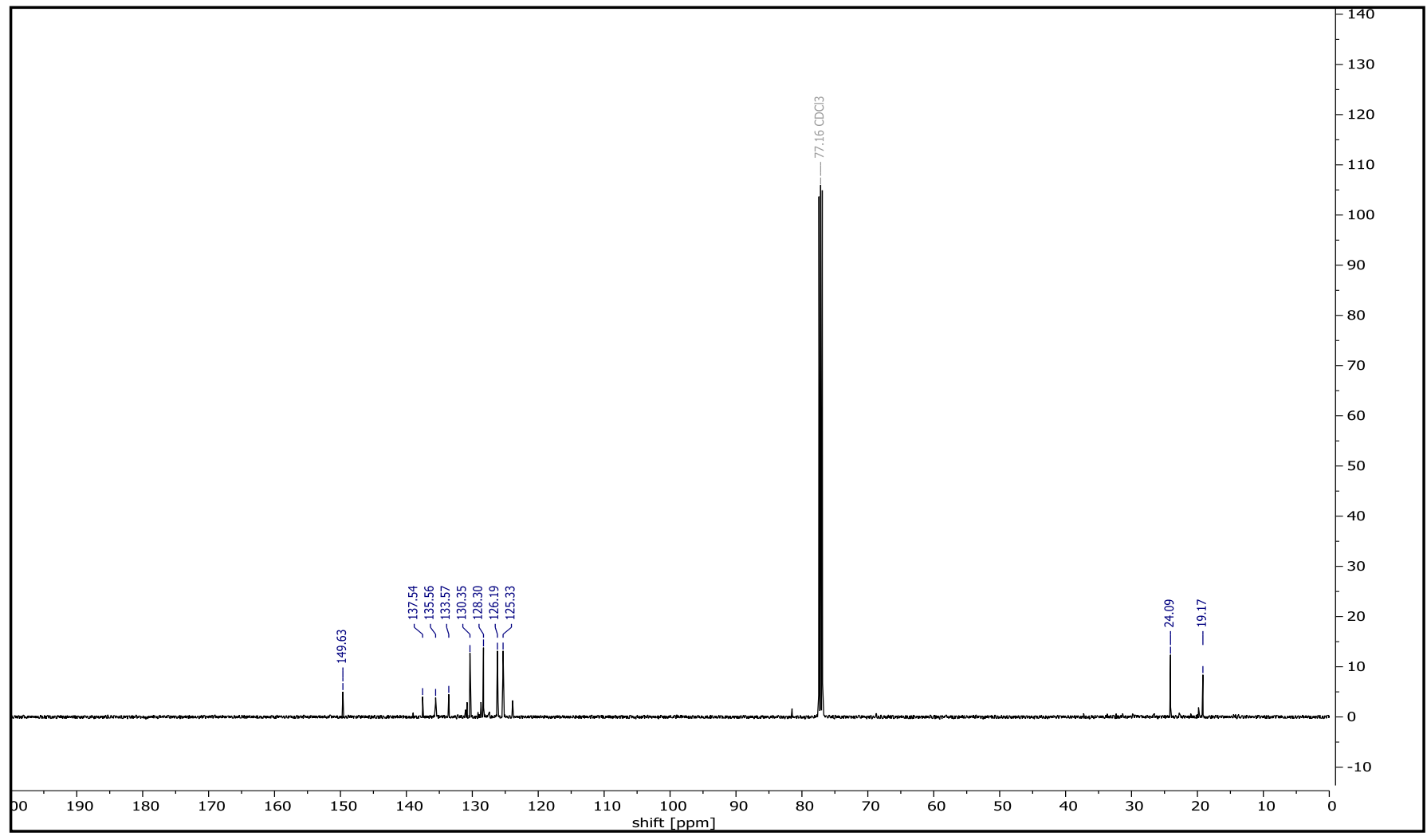


(Z)-1-Methyl-3-(1-nitroprop-1-en-2-yl)benzene (Z-8)

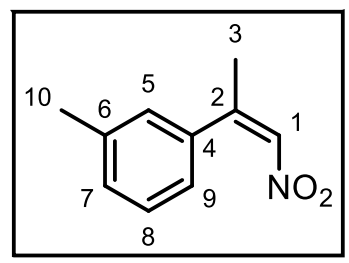

${ }^{1} \mathrm{H}-\mathrm{NMR}\left(600 \mathrm{MHz}, \mathrm{CDCl}_{3}\right)$

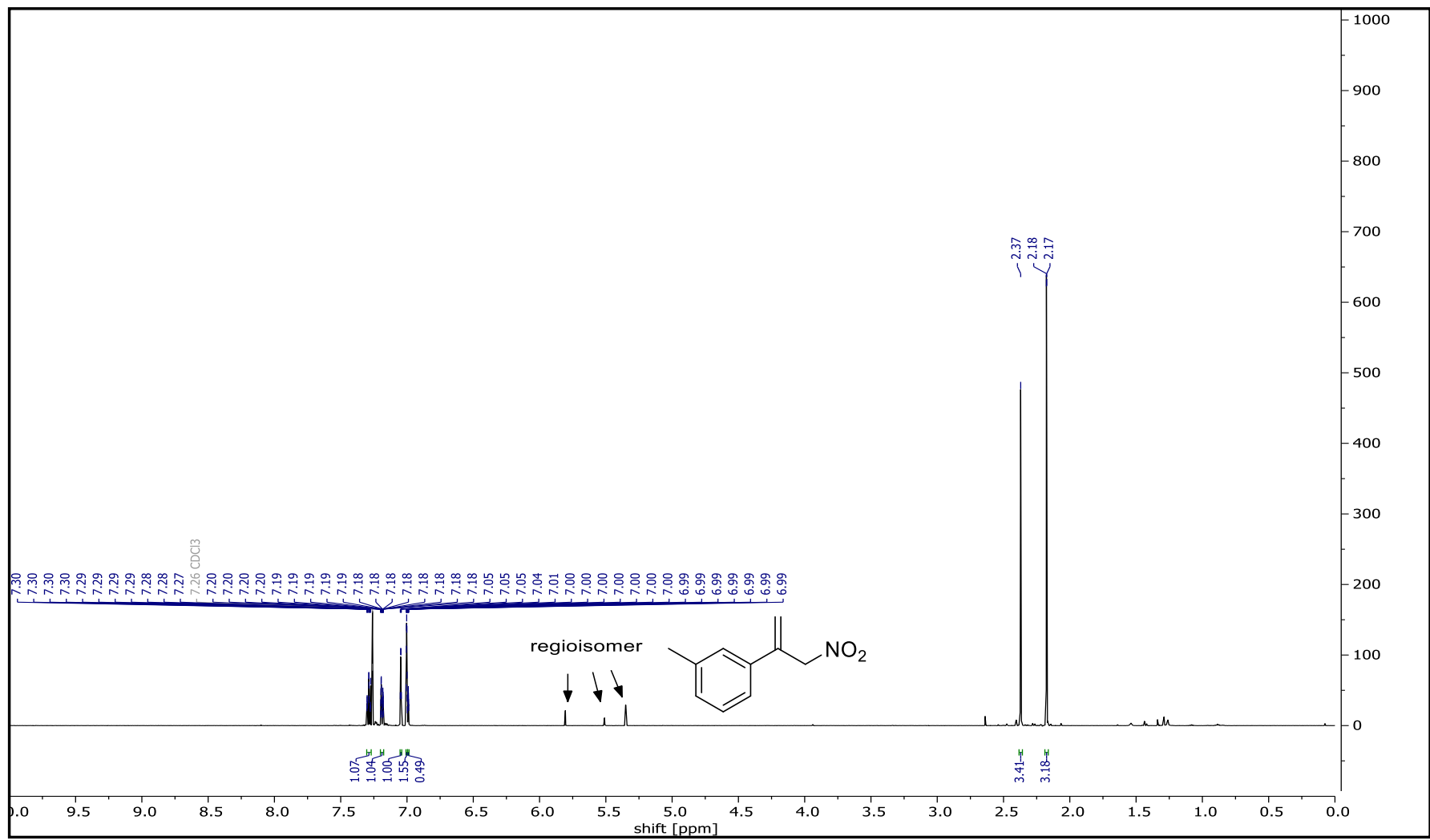

${ }^{13} \mathrm{C}-\mathrm{NMR}\left(151 \mathrm{MHz}, \mathrm{CDCl}_{3}\right)$

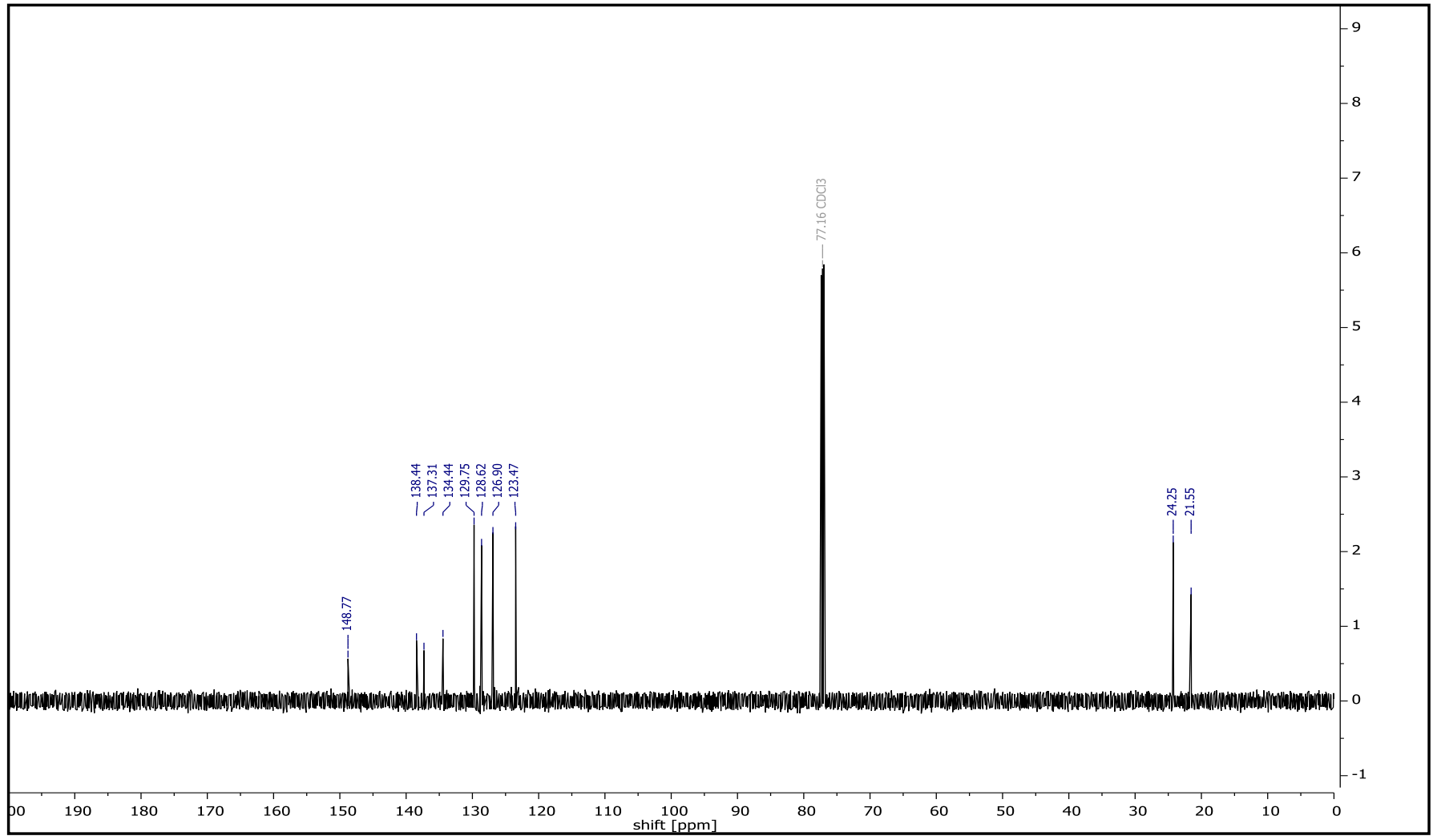


(Z)-1-Chloro-3-(1-nitroprop-1-en-2-yl)benzene (Z-9)

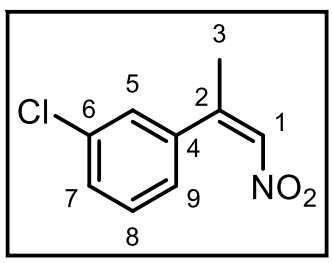

${ }^{1} \mathrm{H}-\mathrm{NMR}\left(300 \mathrm{MHz}, \mathrm{CDCl}_{3}\right)$

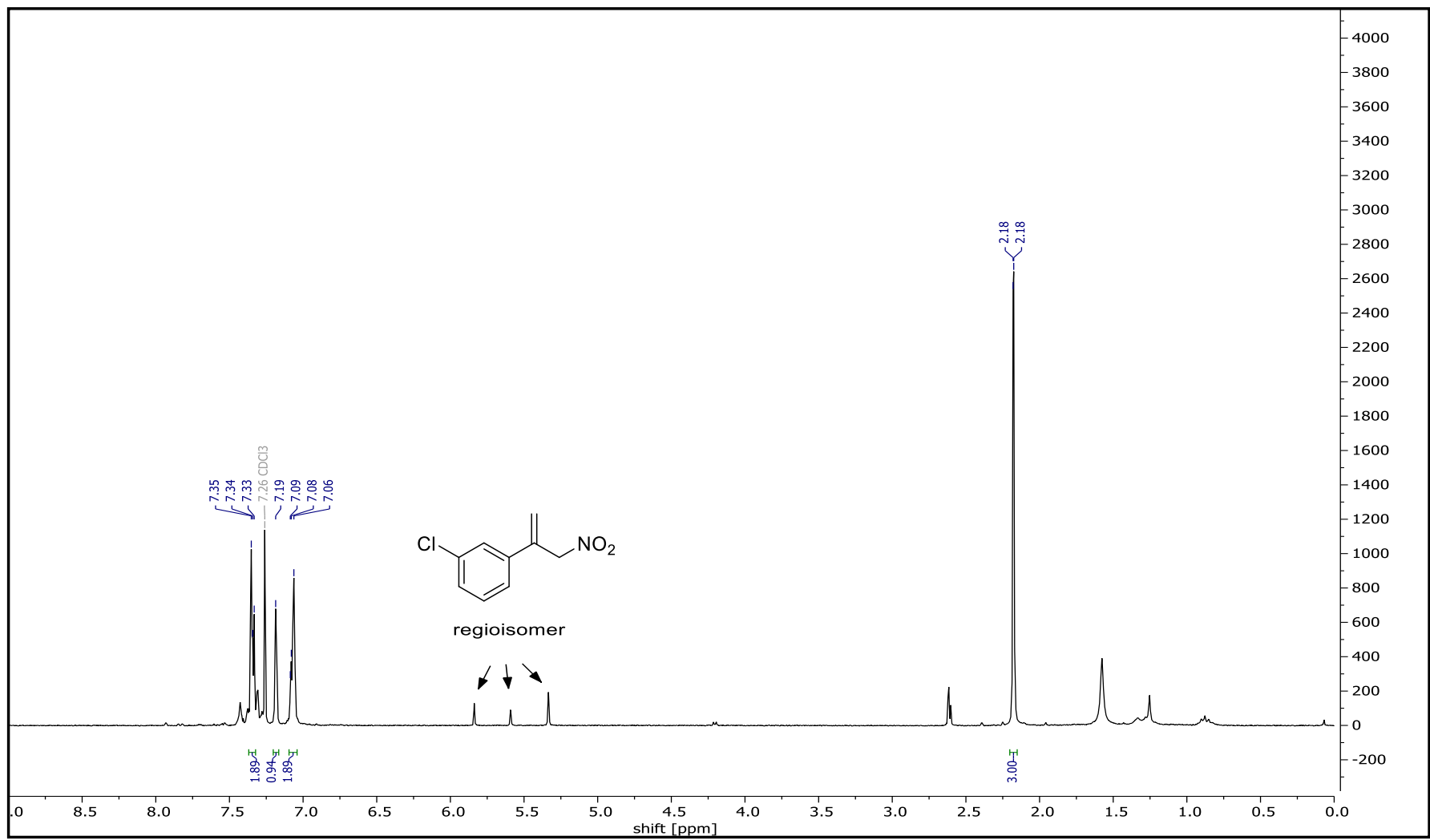

${ }^{13} \mathrm{C}-\mathrm{NMR}\left(101 \mathrm{MHz}, \mathrm{CDCl}_{3}\right)$

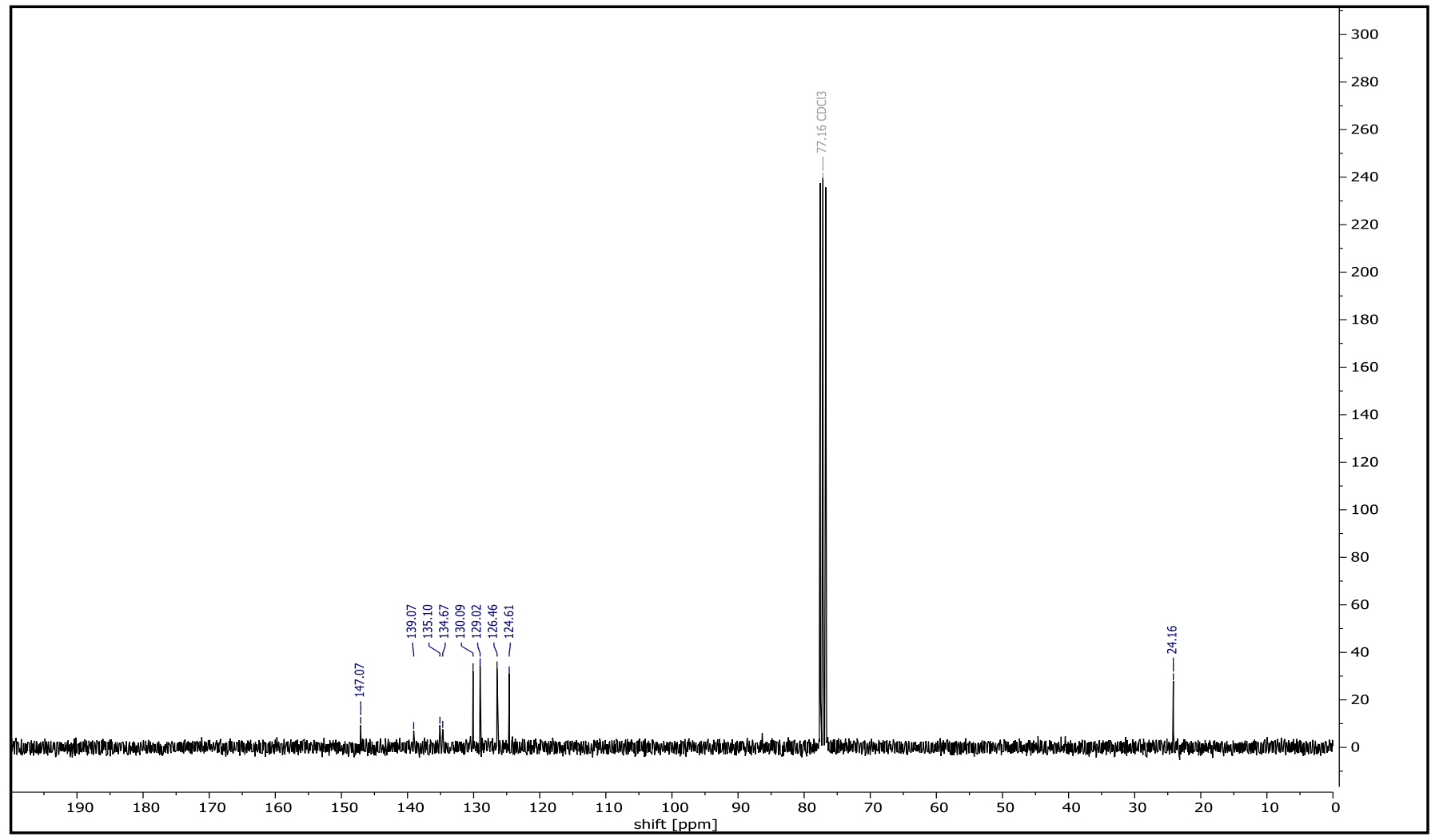


(Z)-1-(Cyclopropyl-2-nitrovinyl)benzene (Z-10)

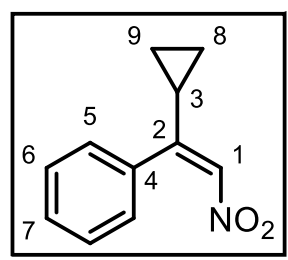

${ }^{1} \mathrm{H}-\mathrm{NMR}\left(500 \mathrm{MHz}, \mathrm{CDCl}_{3}\right)$

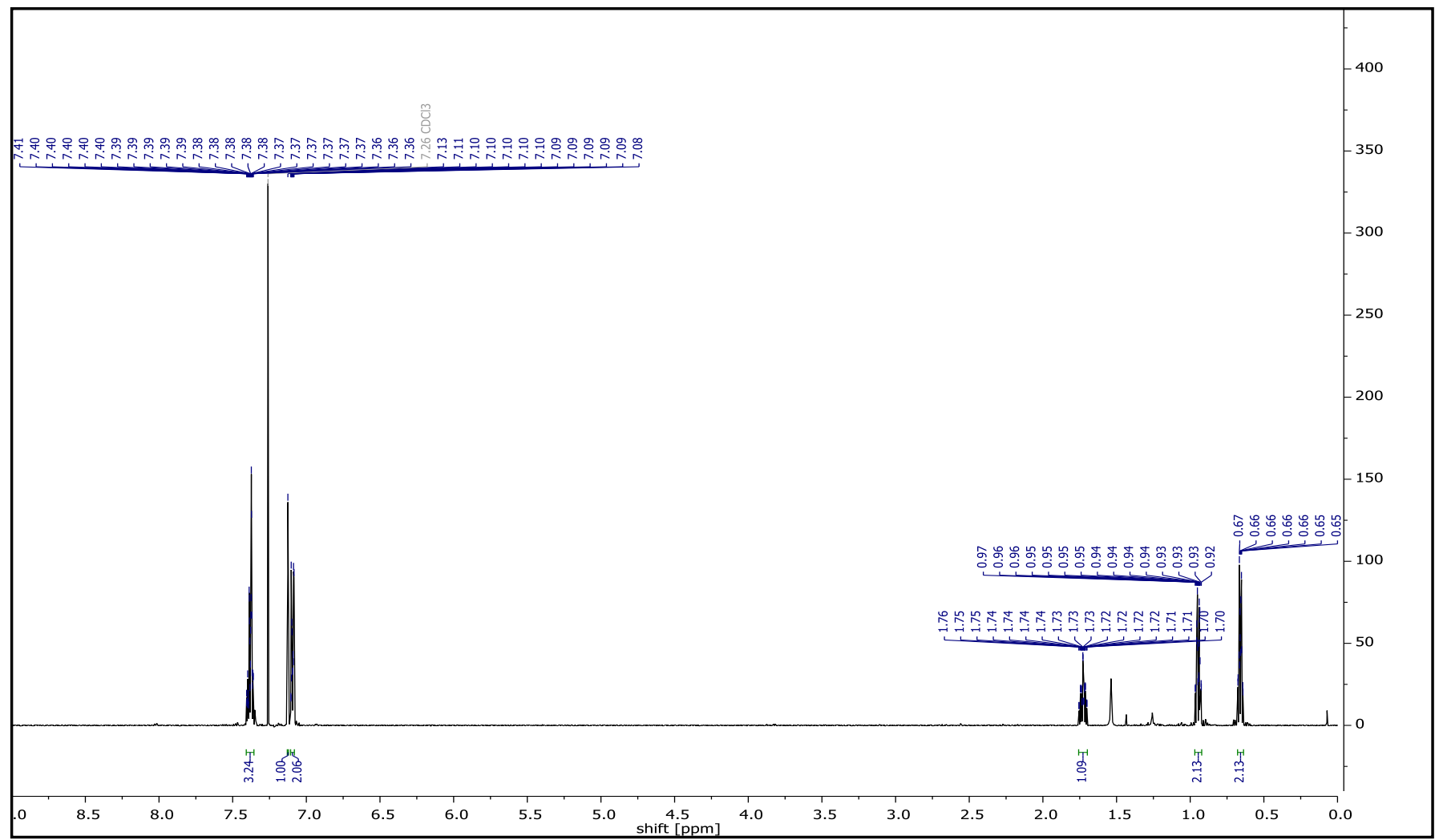

${ }^{13} \mathrm{C}-\mathrm{NMR}\left(101 \mathrm{MHz}, \mathrm{CDCl}_{3}\right)$

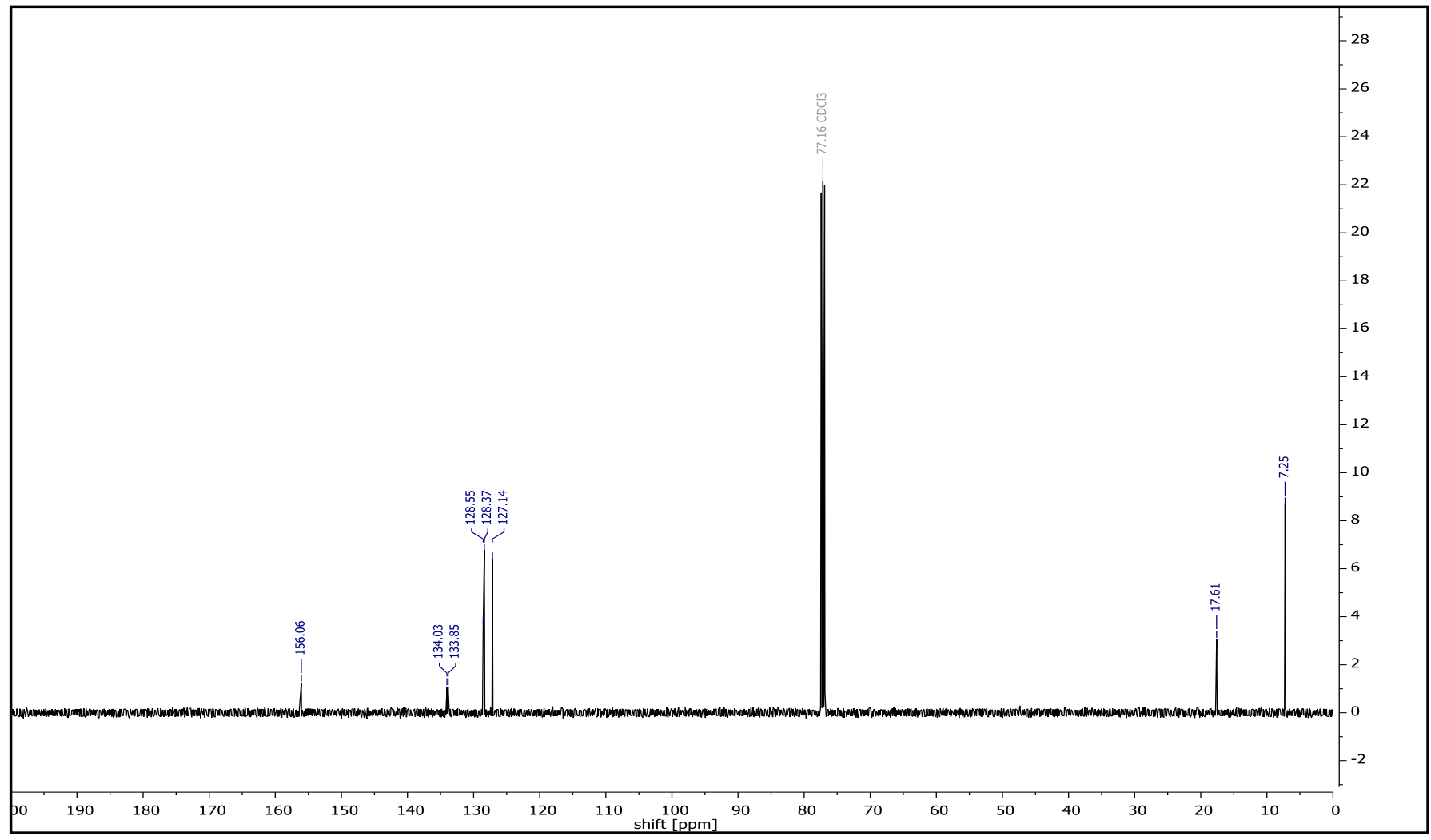


( $Z$ and $E)$-(2-Nitrovinyl)benzene ( $Z$ and $E-11)$

Isomerization led to an inseparable mixture of $Z$ and $E$ isomers after column chromatography as indicated by ${ }^{1} \mathrm{H}-\mathrm{NMR}$

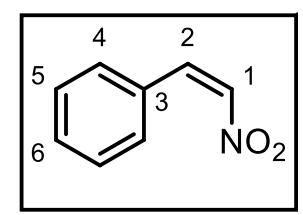

${ }^{1} \mathrm{H}-\mathrm{NMR}\left(400 \mathrm{MHz}, \mathrm{CDCl}_{3}\right)$

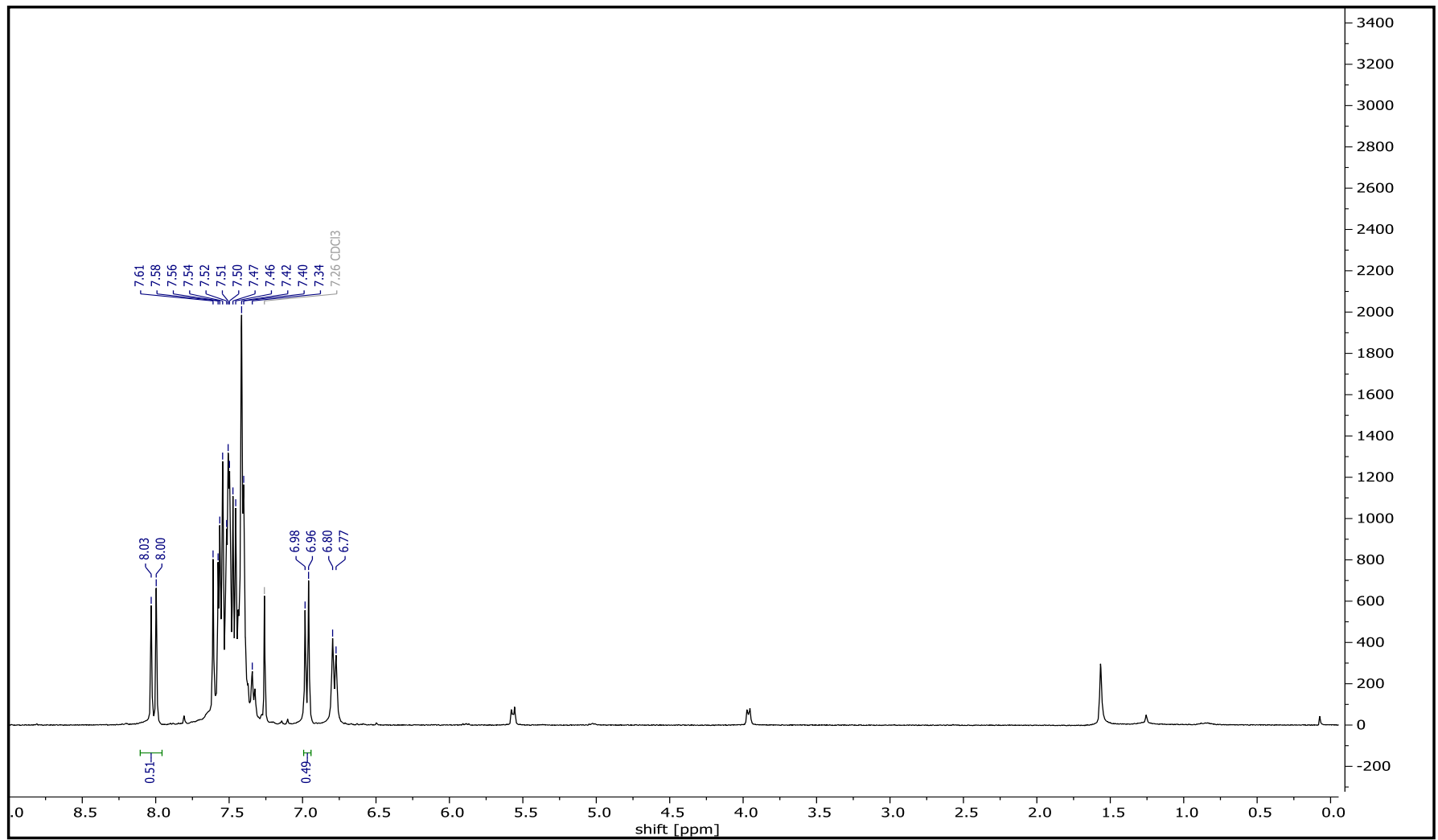


( $Z$ and $E$ )-1-Fluoro-2-(2-nitrovinyl)benzene ( $Z$ - and $E$-12)

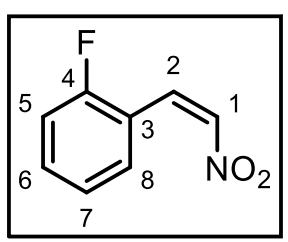

${ }^{1} \mathrm{H}-\mathrm{NMR}\left(600 \mathrm{MHz}, \mathrm{CDCl}_{3}\right)$

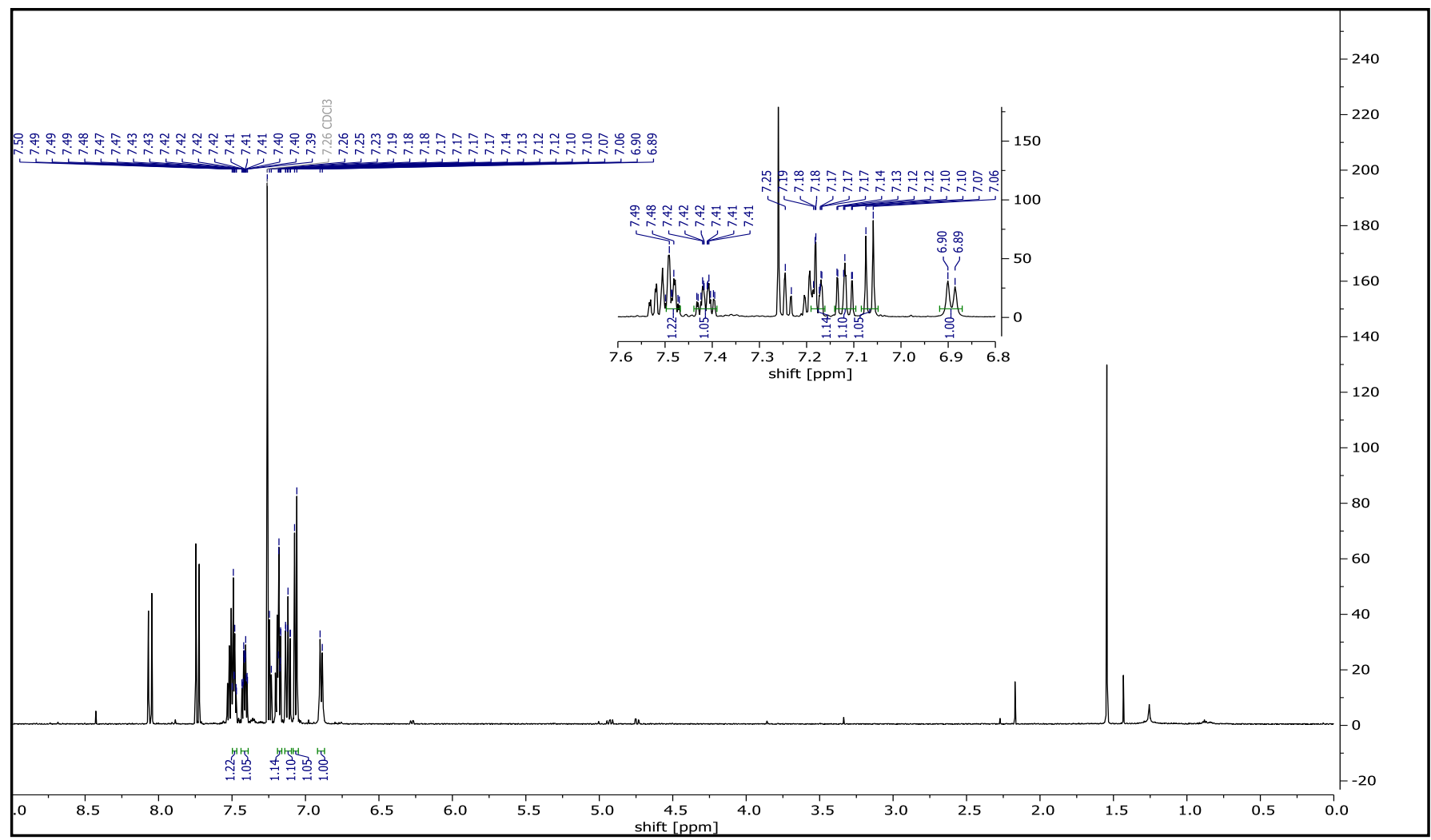

${ }^{13} \mathrm{C}-\mathrm{NMR}\left(151 \mathrm{MHz}, \mathrm{CDCl}_{3}\right)$

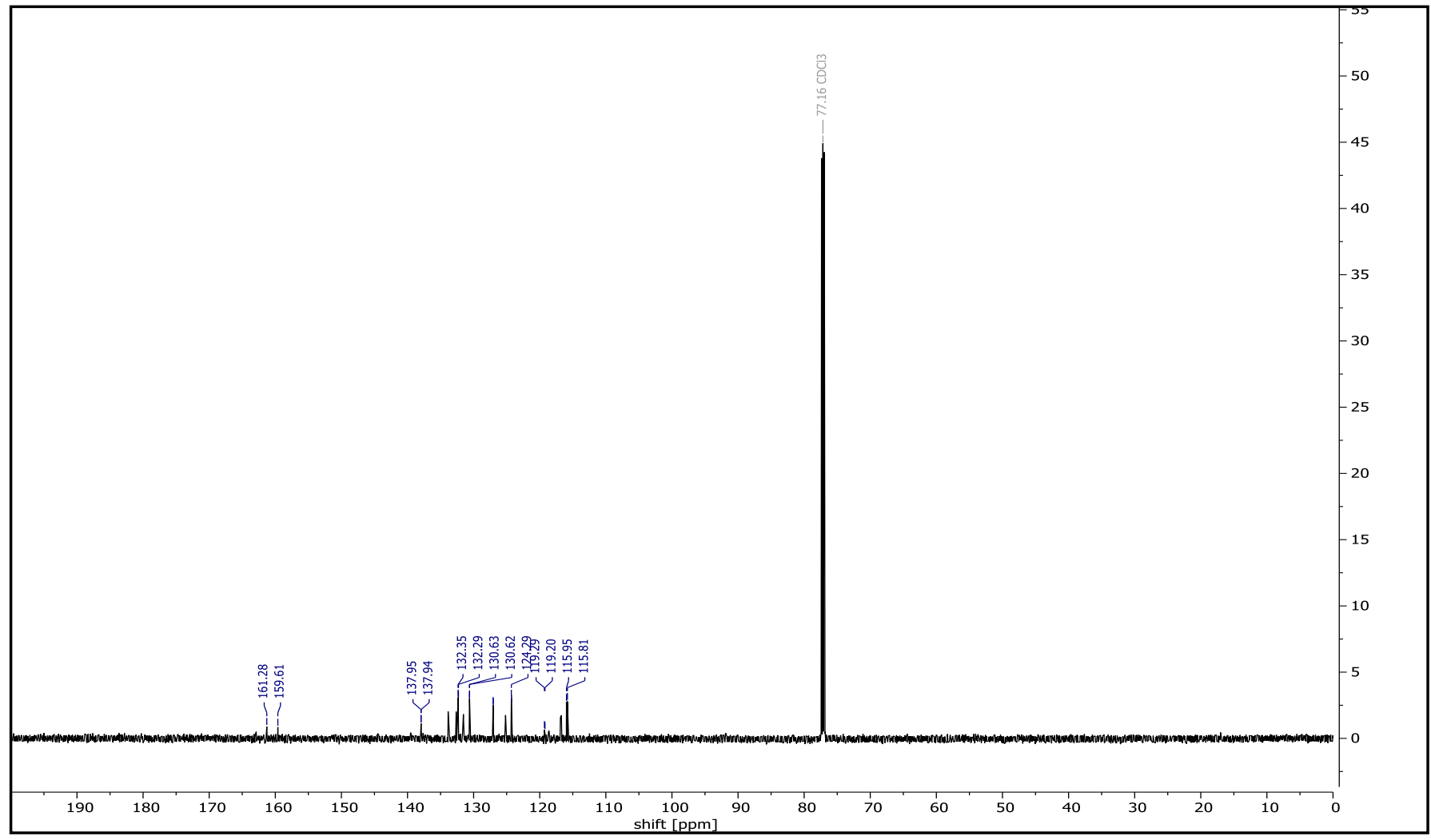


( $Z$ and $E$ )-1-Fluoro-2-(2-nitrovinyl)benzene ( $Z$ - and $E$-12)

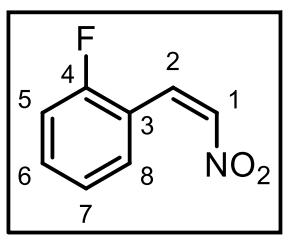

${ }^{19} \mathrm{~F}-\mathrm{NMR}\left(564 \mathrm{MHz}, \mathrm{CDCl}_{3}\right)$

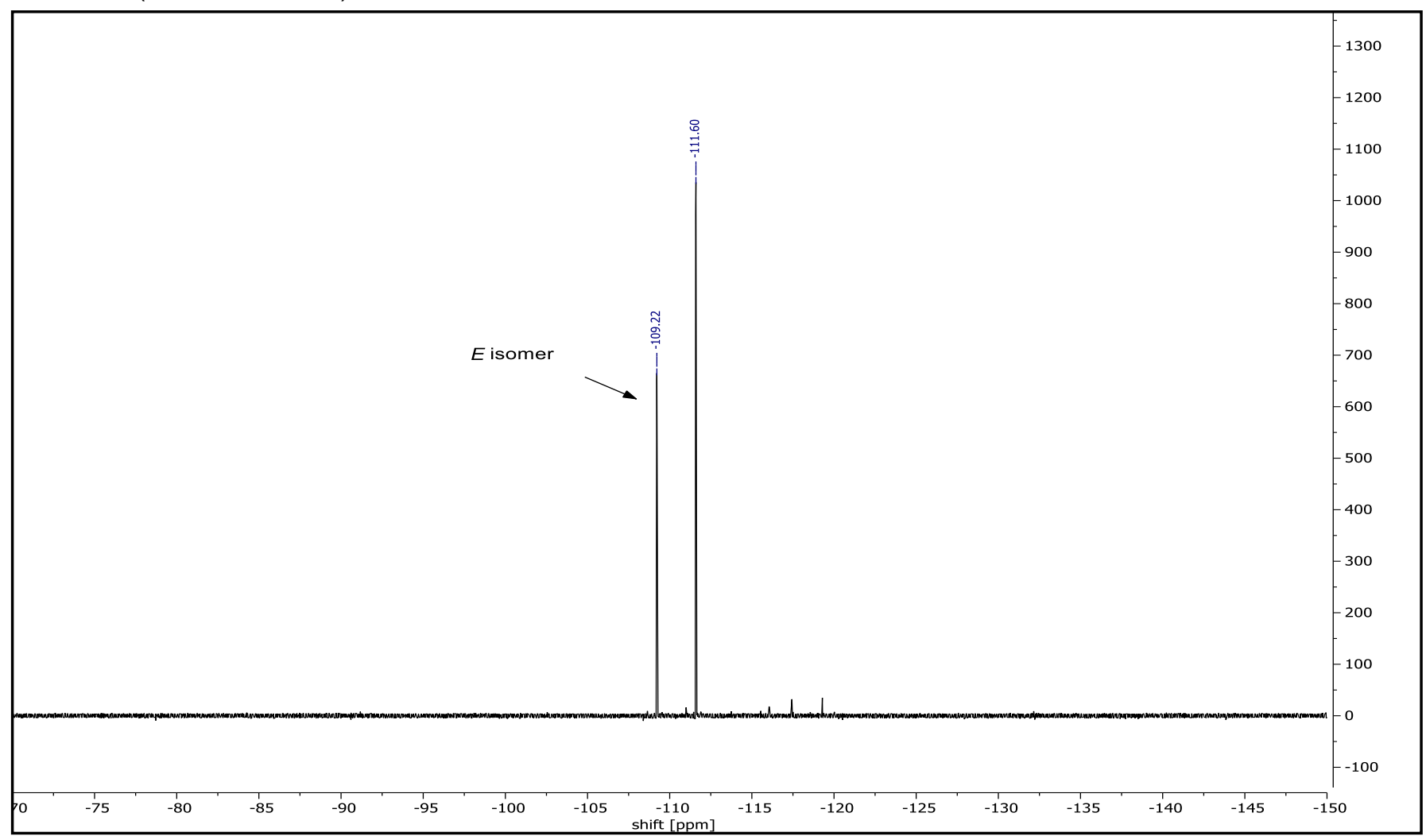


(1-Nitropropan-2-yl)benzene (13)

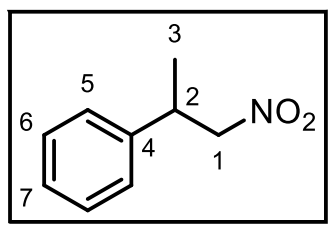

${ }^{1} \mathrm{H}-\mathrm{NMR}\left(400 \mathrm{MHz}, \mathrm{CDCl}_{3}\right)$

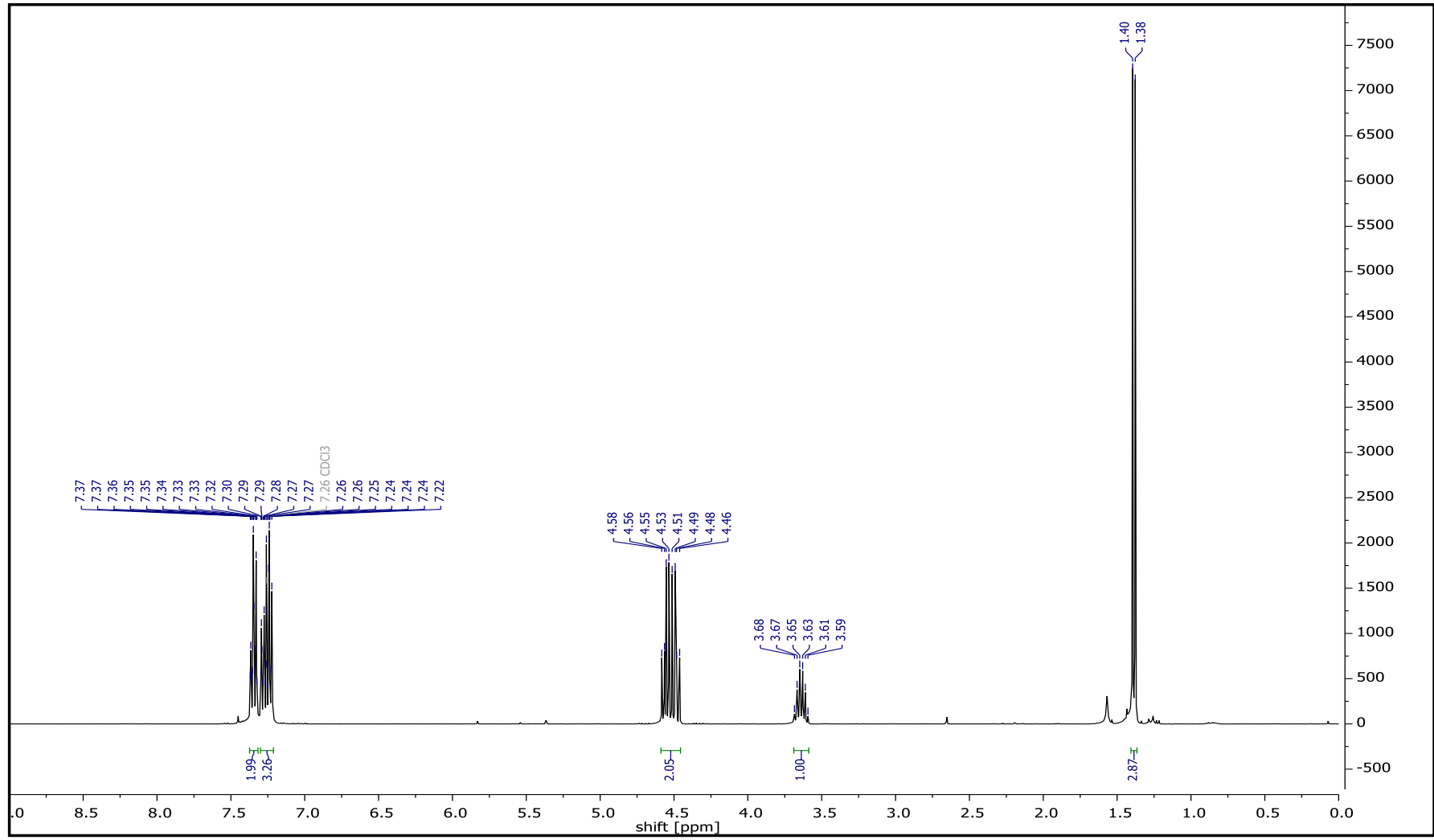

${ }^{13} \mathrm{C}-\mathrm{NMR}\left(101 \mathrm{MHz}, \mathrm{CDCl}_{3}\right)$

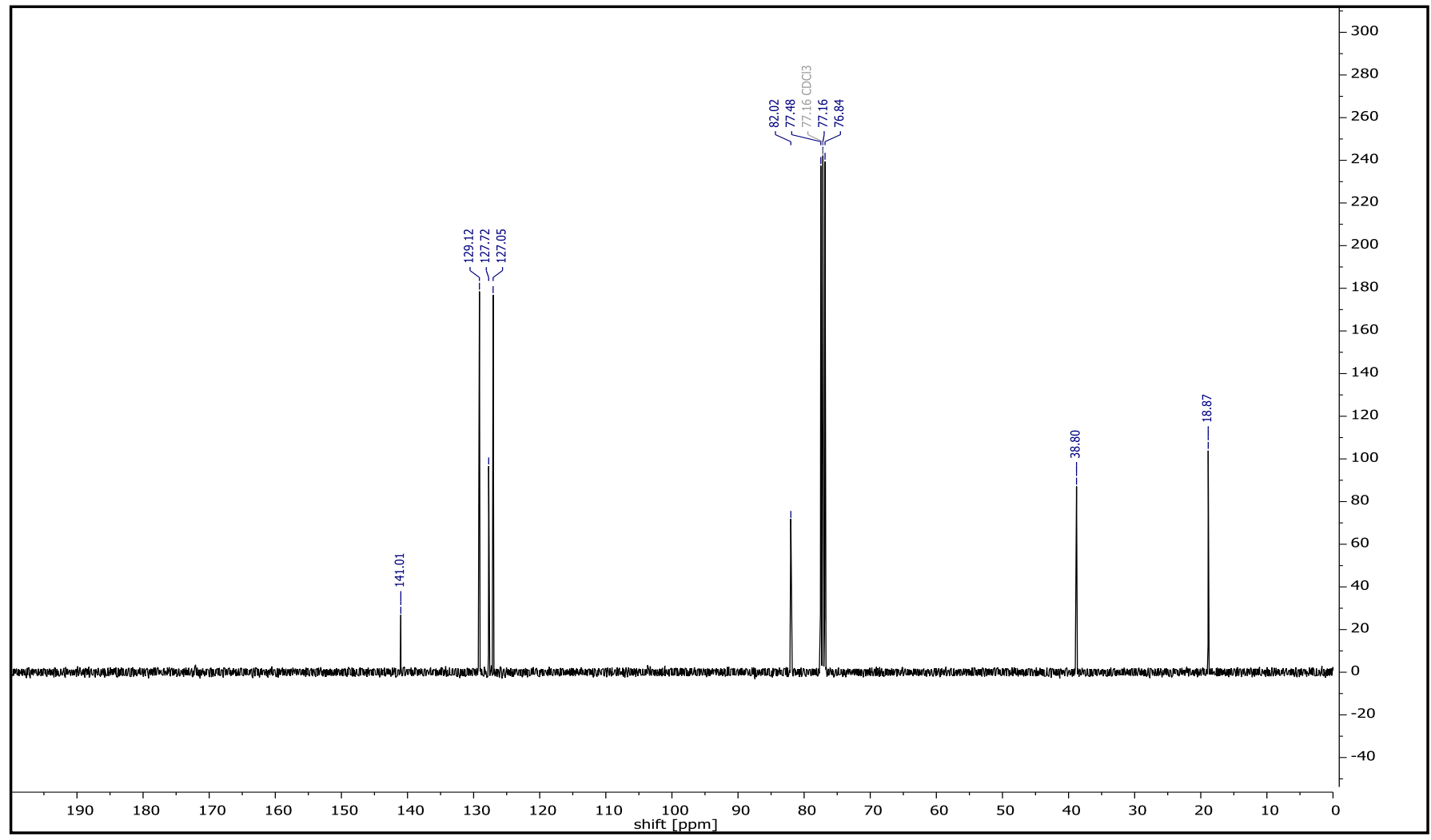


1-Methyl-4-(1-nitropropan-2-yl)benzene (14)

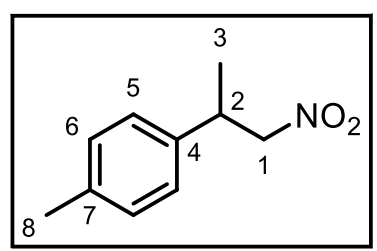

${ }^{1} \mathrm{H}-\mathrm{NMR}\left(400 \mathrm{MHz}, \mathrm{CDCl}_{3}\right)$

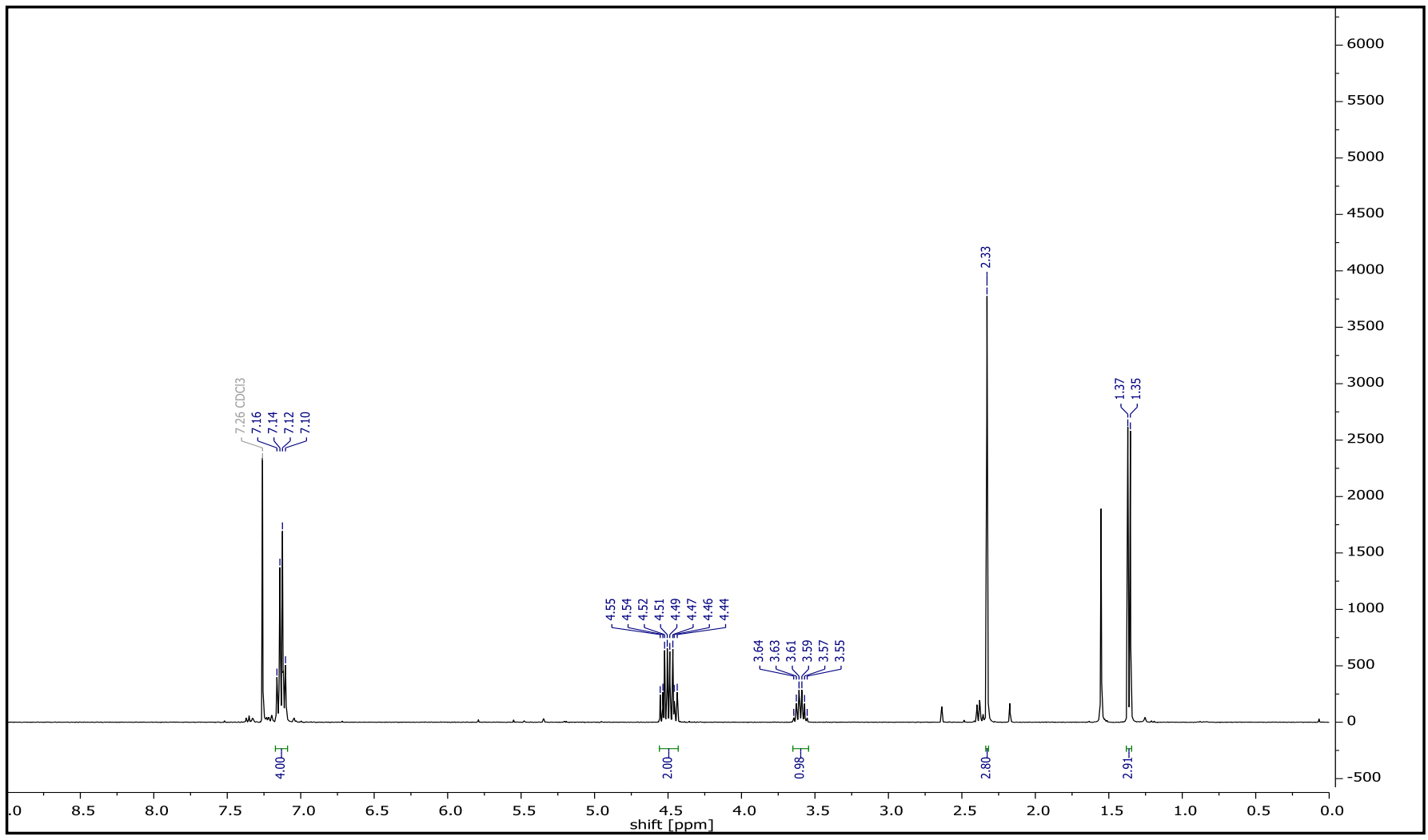

${ }^{13} \mathrm{C}-\mathrm{NMR}\left(101 \mathrm{MHz}, \mathrm{CDCl}_{3}\right)$

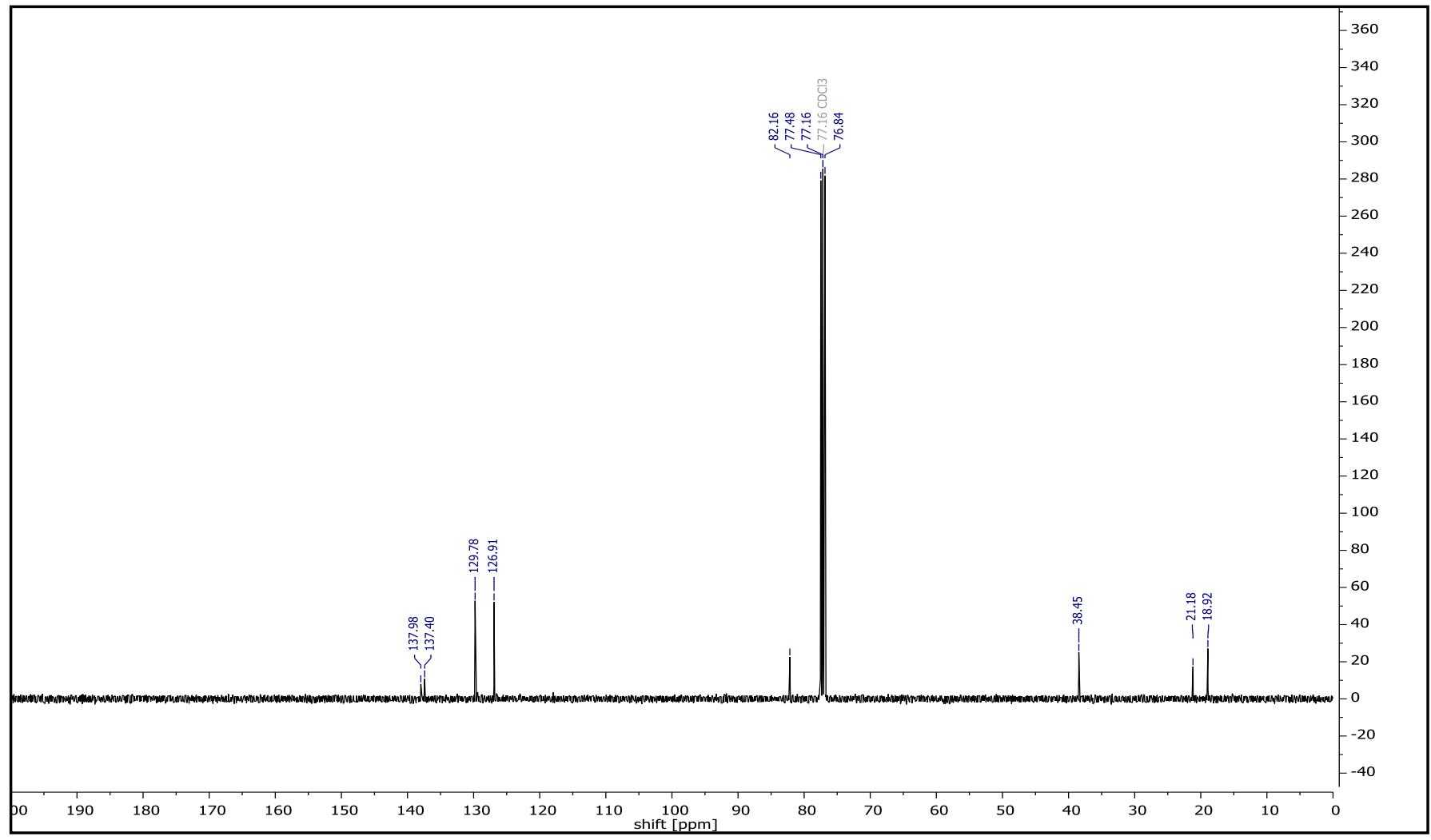


1-Methoxy-4-(1-nitropropan-2-yl)benzene (15)

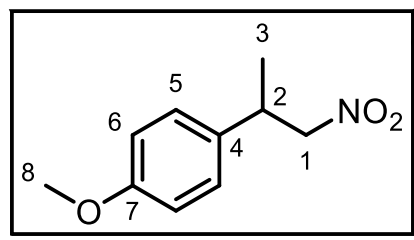

${ }^{1} \mathrm{H}-\mathrm{NMR}\left(400 \mathrm{MHz}, \mathrm{CDCl}_{3}\right)$

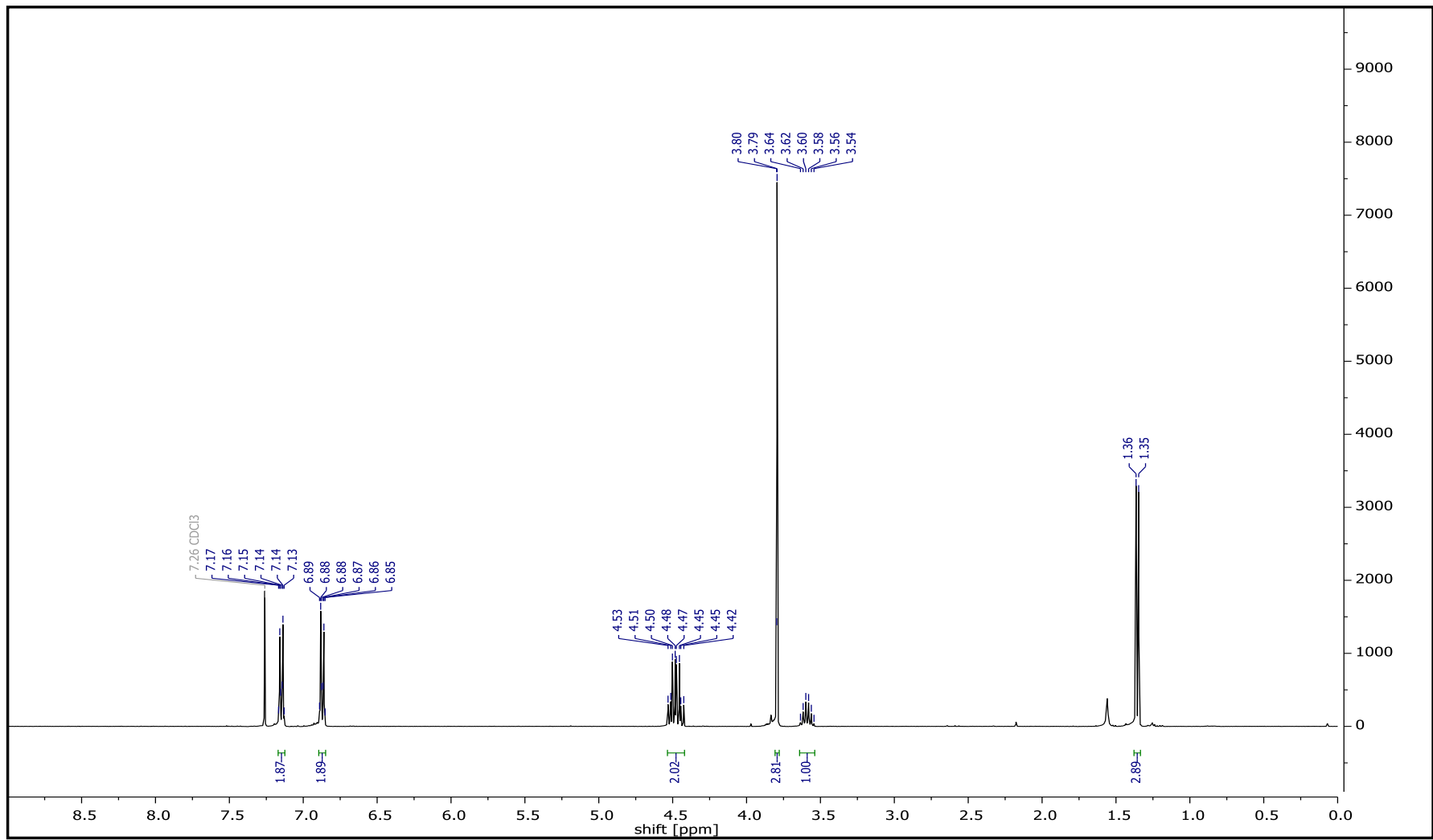

${ }^{13} \mathrm{C}-\mathrm{NMR}\left(101 \mathrm{MHz}, \mathrm{CDCl}_{3}\right)$

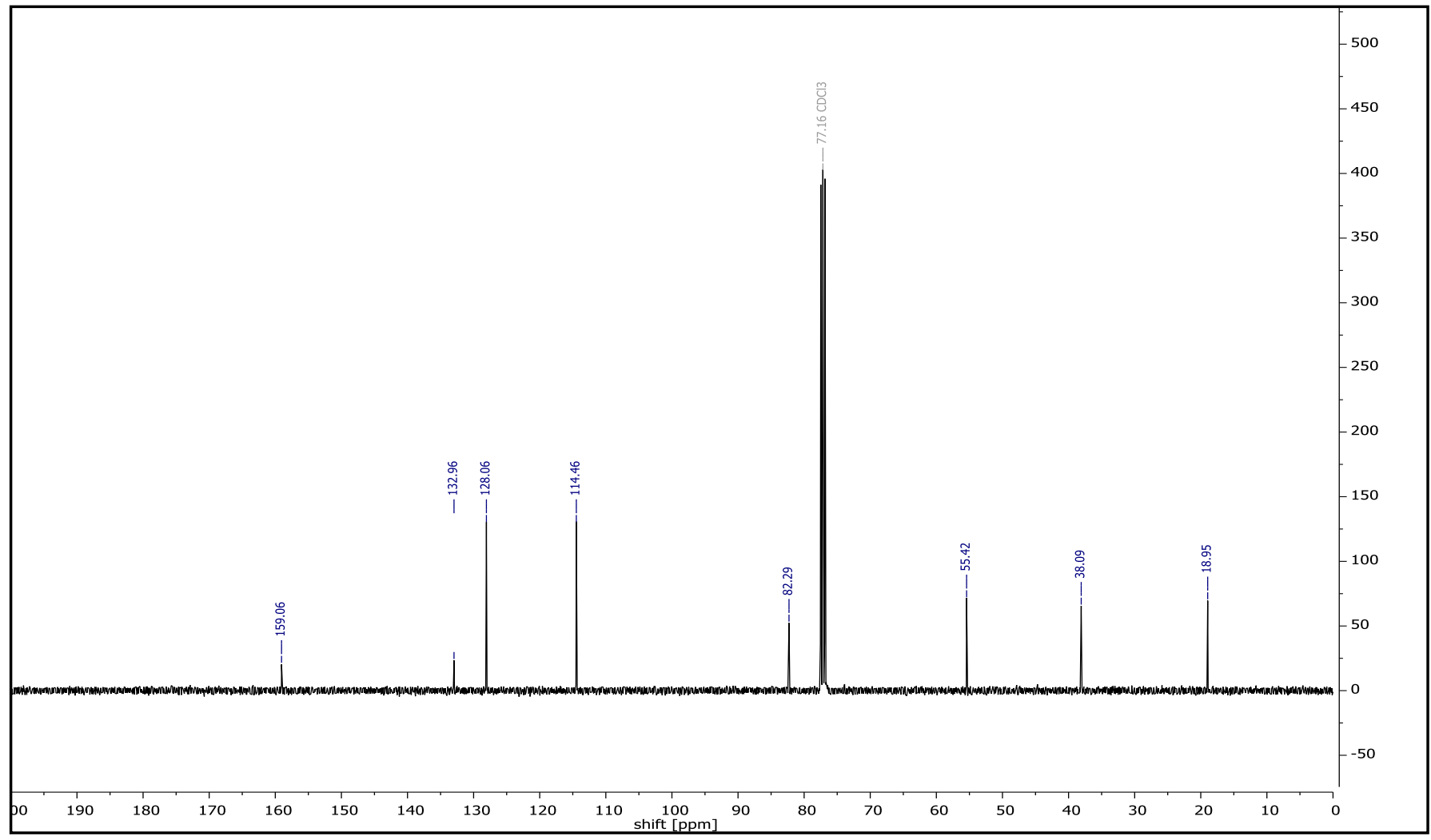


1-Fluoro-4-(1-nitropropan-2-yl)benzene (16)

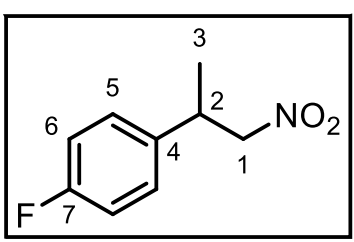

${ }^{1} \mathrm{H}-\mathrm{NMR}\left(400 \mathrm{MHz}, \mathrm{CDCl}_{3}\right)$

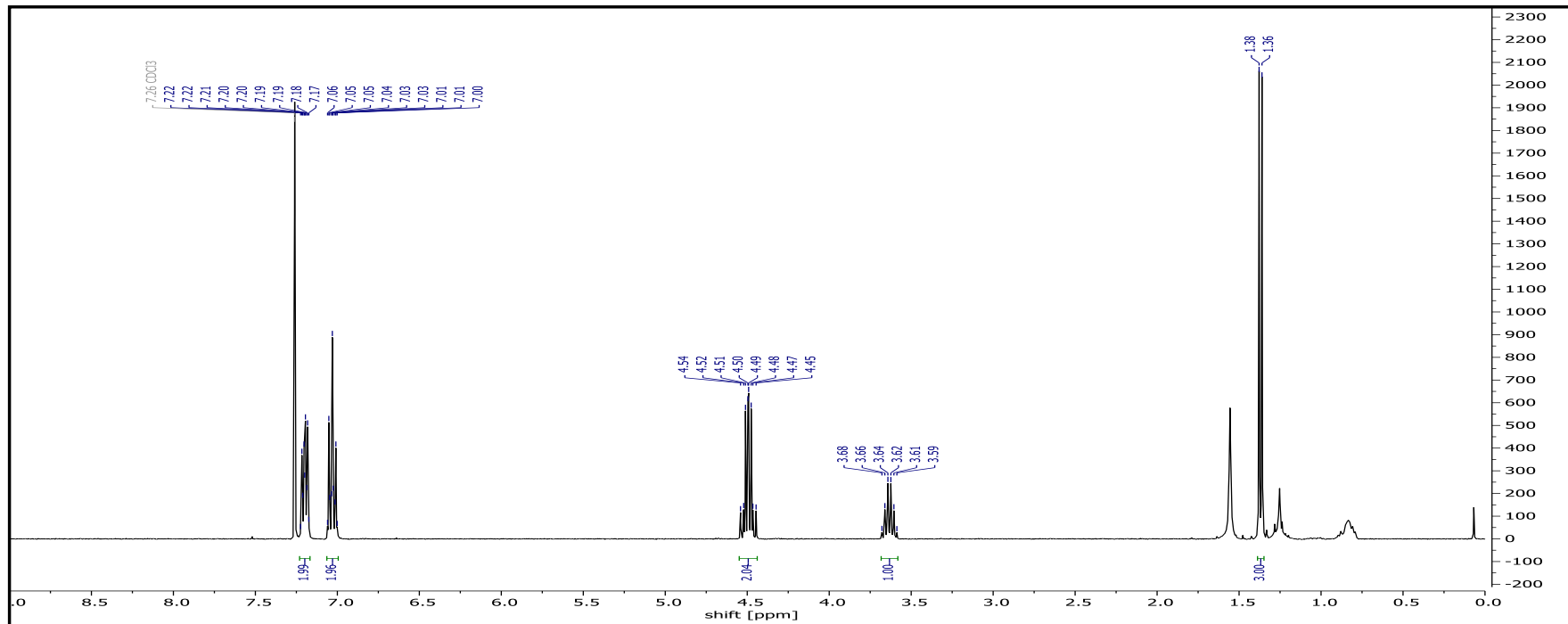

${ }^{13} \mathrm{C}-\mathrm{NMR}\left(101 \mathrm{MHz}, \mathrm{CDCl}_{3}\right)$

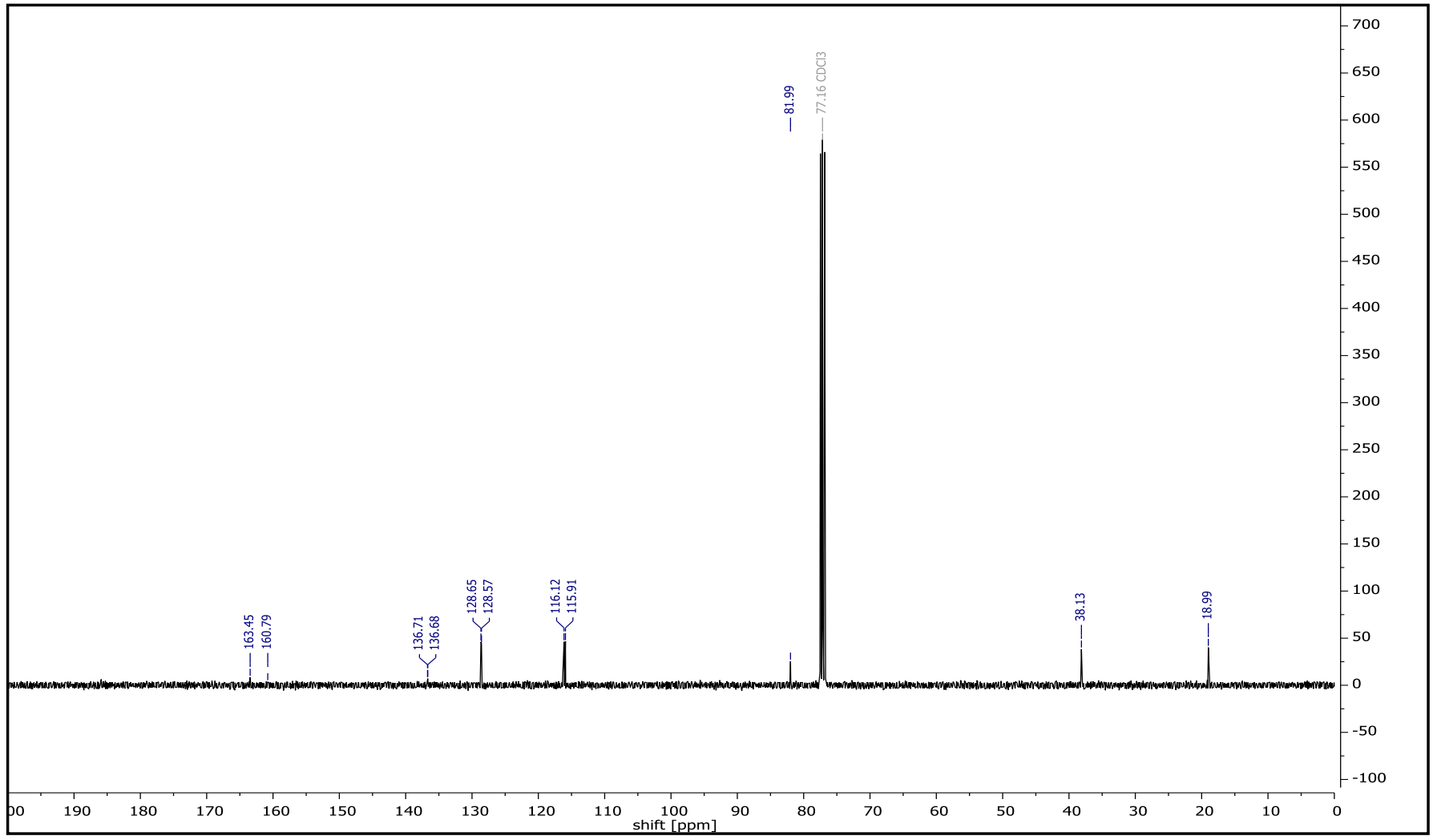


1-Fluoro-4-(1-nitropropan-2-yl)benzene (16)

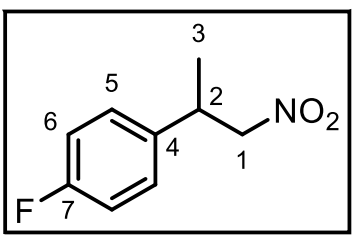

${ }^{19} \mathrm{~F}-\mathrm{NMR}\left(376 \mathrm{MHz}, \mathrm{CDCl}_{3}\right)$

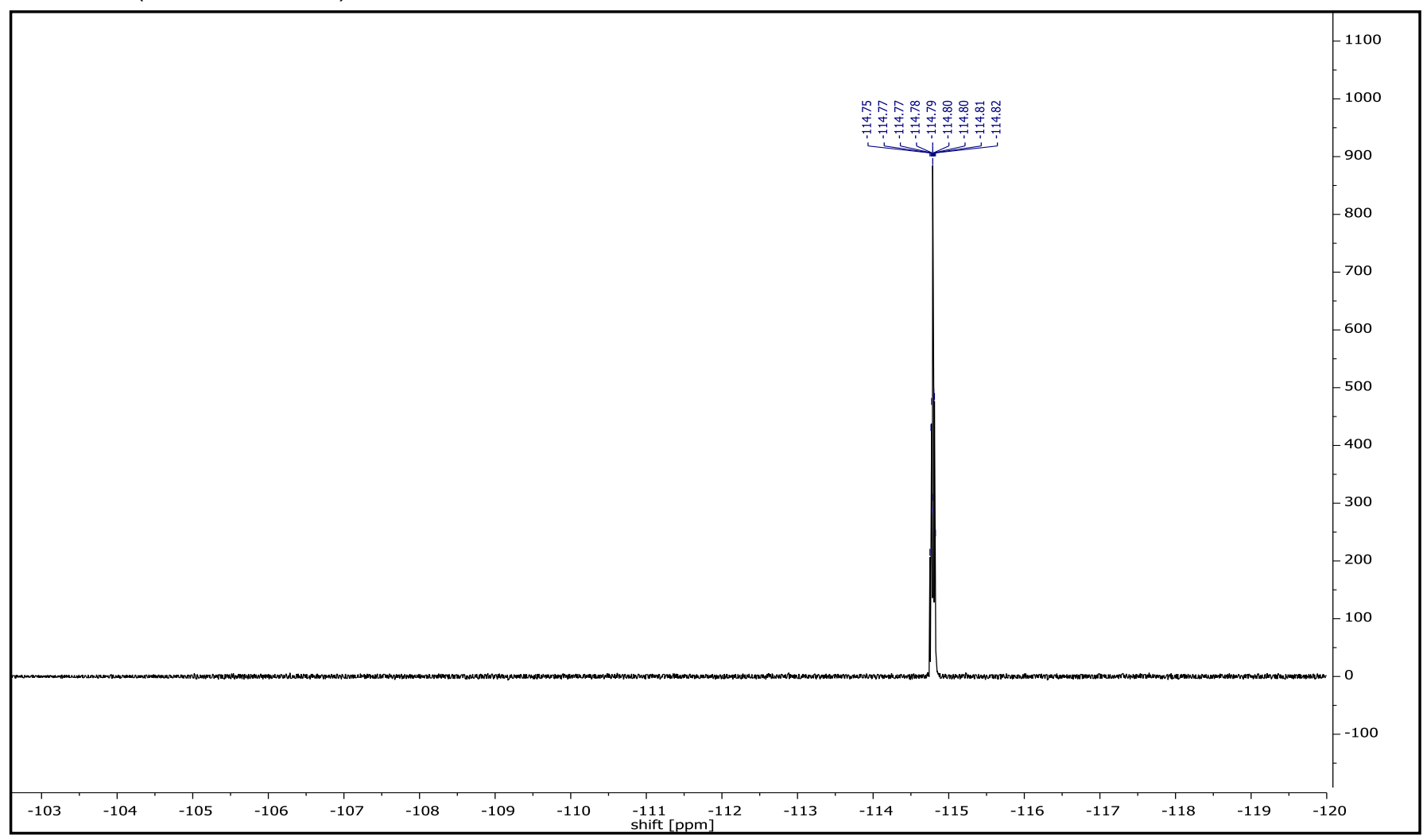




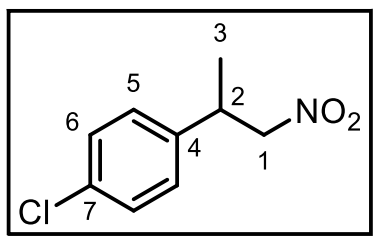

${ }^{1} \mathrm{H}-\mathrm{NMR}\left(400 \mathrm{MHz}, \mathrm{CDCl}_{3}\right)$

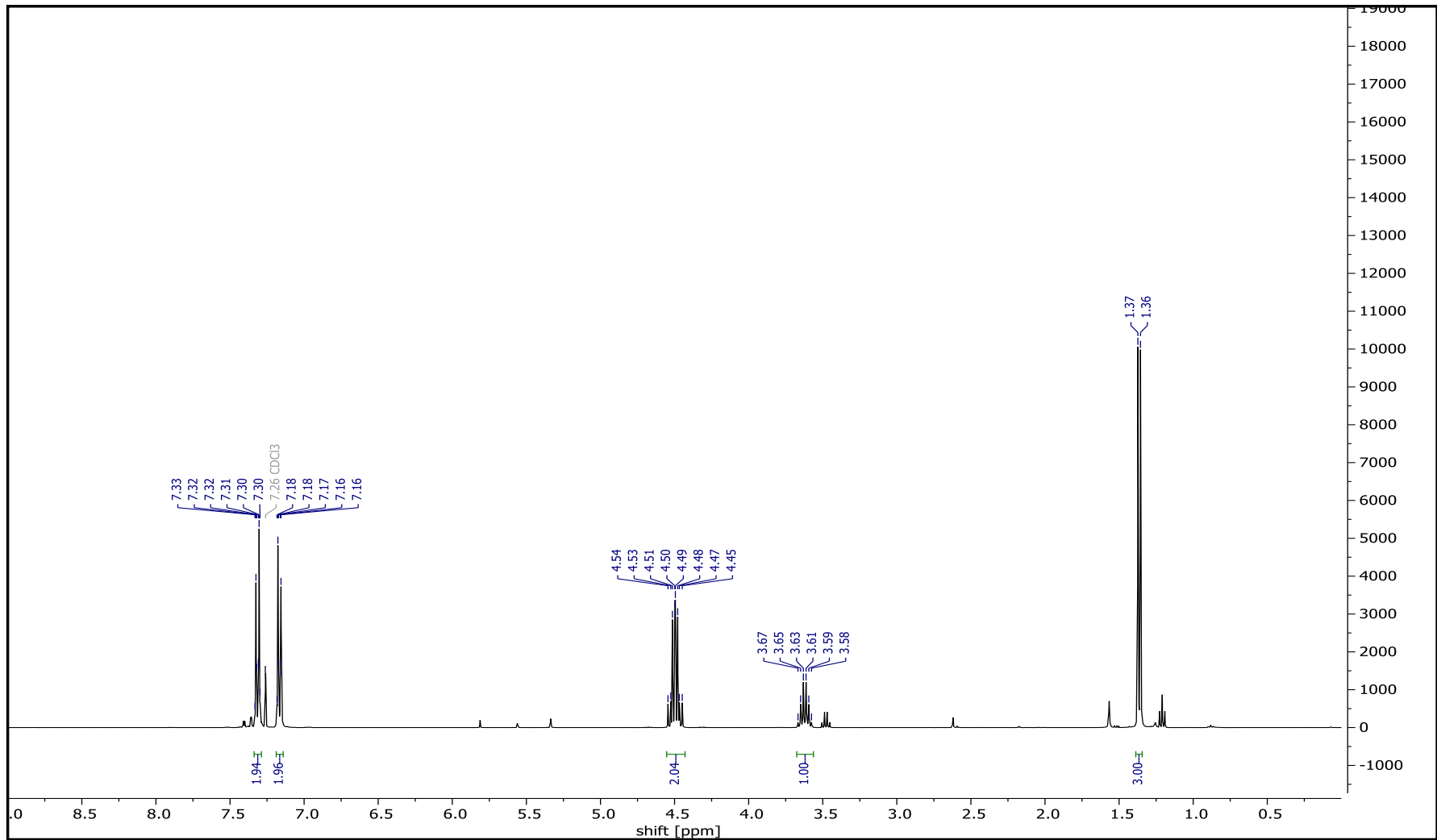

${ }^{13} \mathrm{C}-\mathrm{NMR}\left(101 \mathrm{MHz}, \mathrm{CDCl}_{3}\right)$

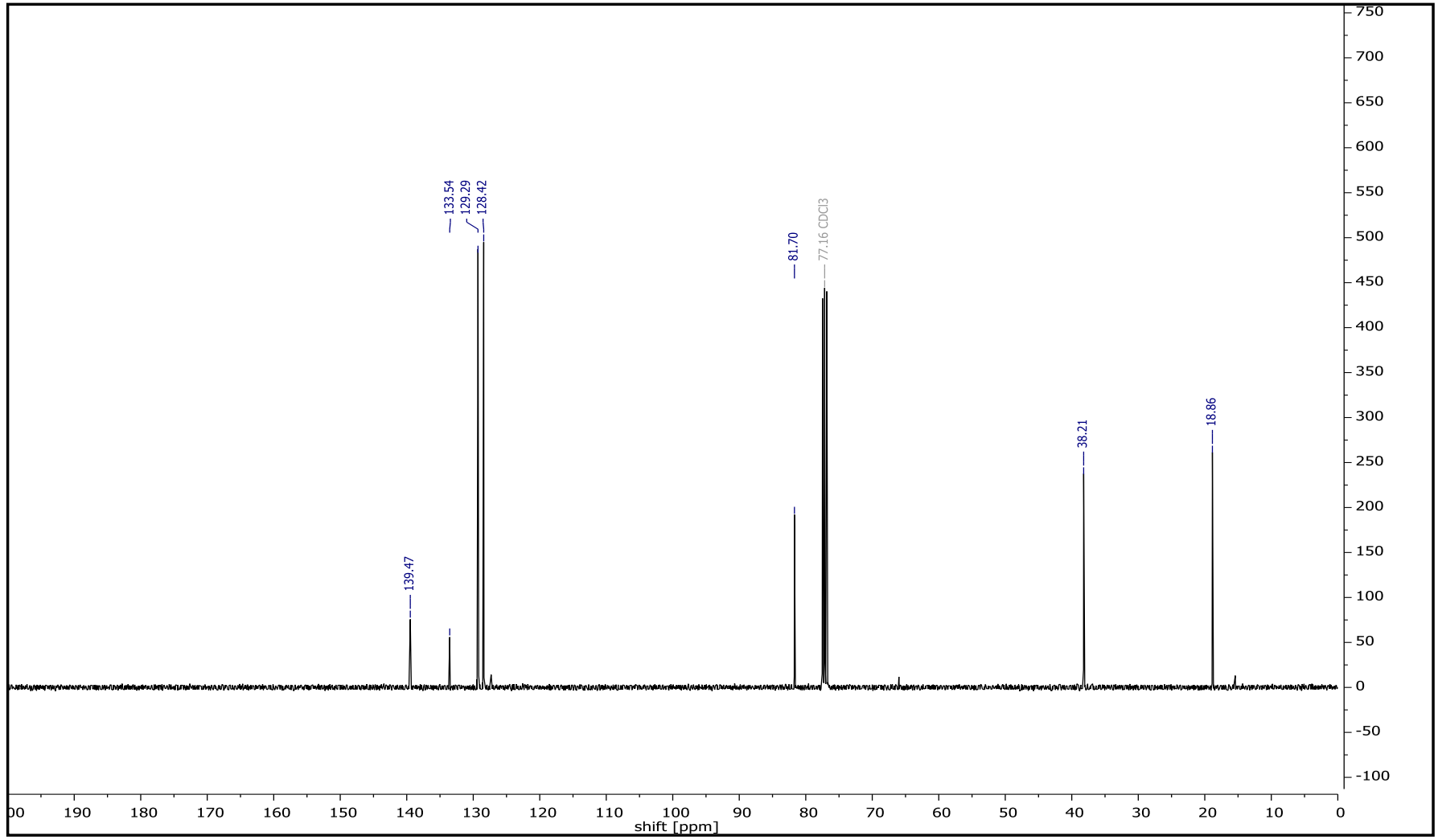


1-(1-Nitropropan-2-yl)-4-(trifluoromethyl)benzene (18)

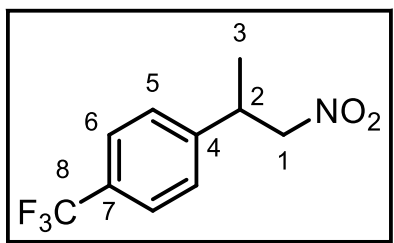

${ }^{1} \mathrm{H}-\mathrm{NMR}\left(400 \mathrm{MHz}, \mathrm{CDCl}_{3}\right)$

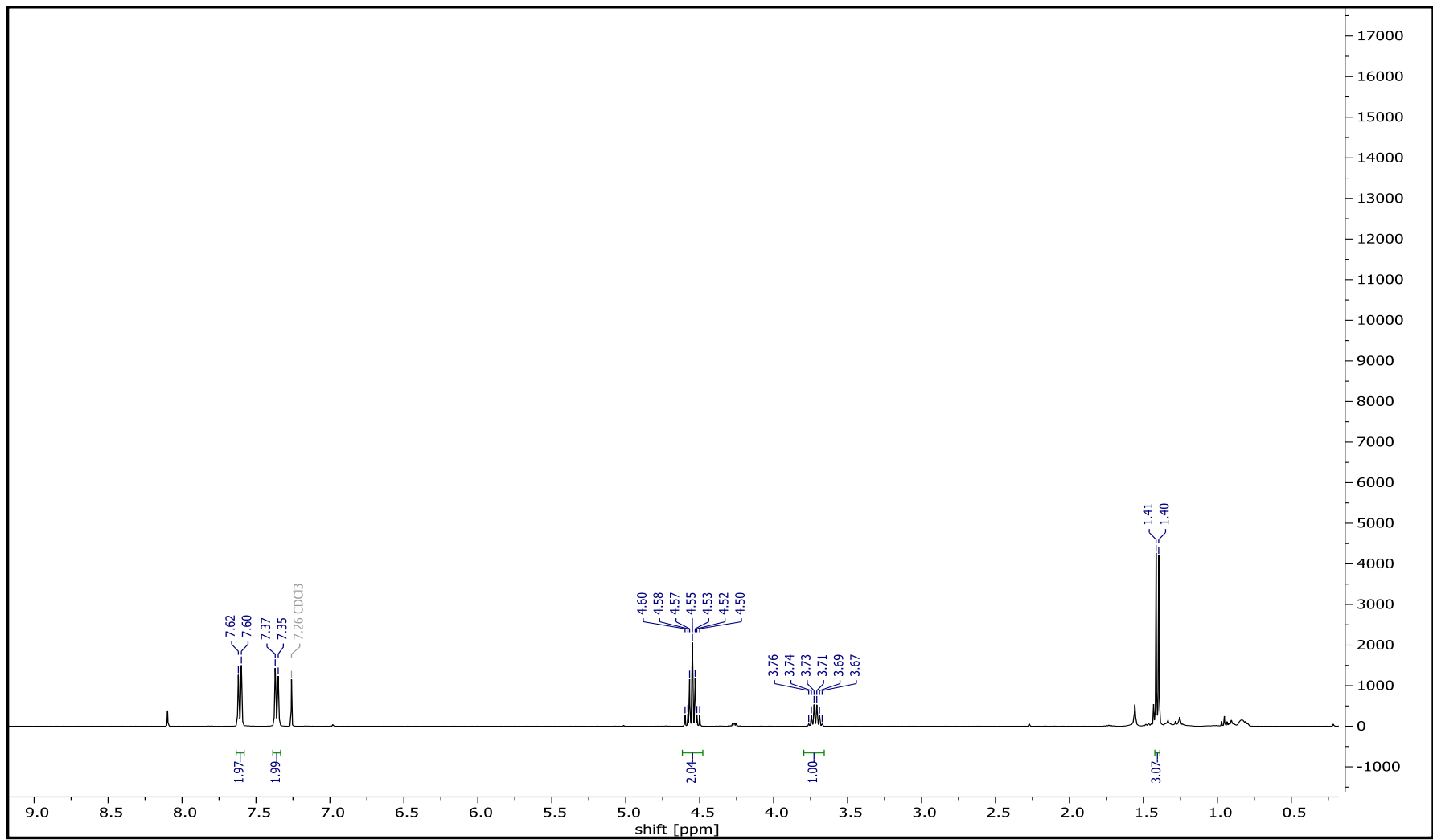

${ }^{13} \mathrm{C}-\mathrm{NMR}\left(101 \mathrm{MHz}, \mathrm{CDCl}_{3}\right)$

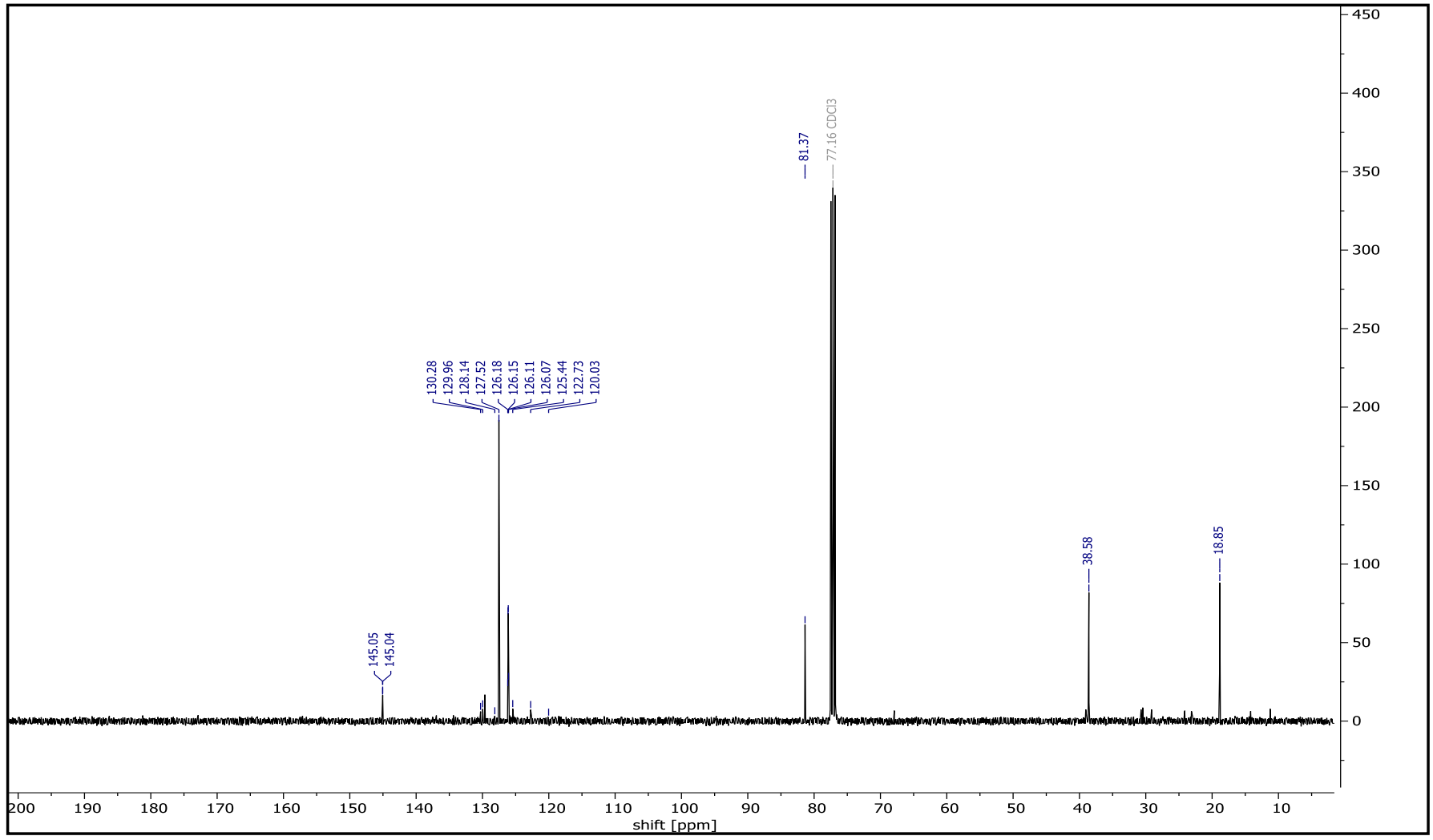


1-(1-Nitropropan-2-yl)-4-(trifluoromethyl)benzene (18)

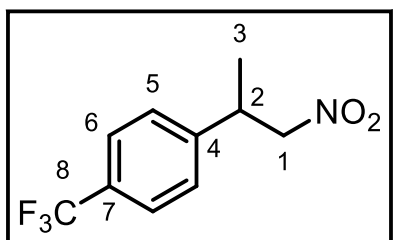

${ }^{19} \mathrm{~F}-\mathrm{NMR}\left(376 \mathrm{MHz}, \mathrm{CDCl}_{3}\right)$

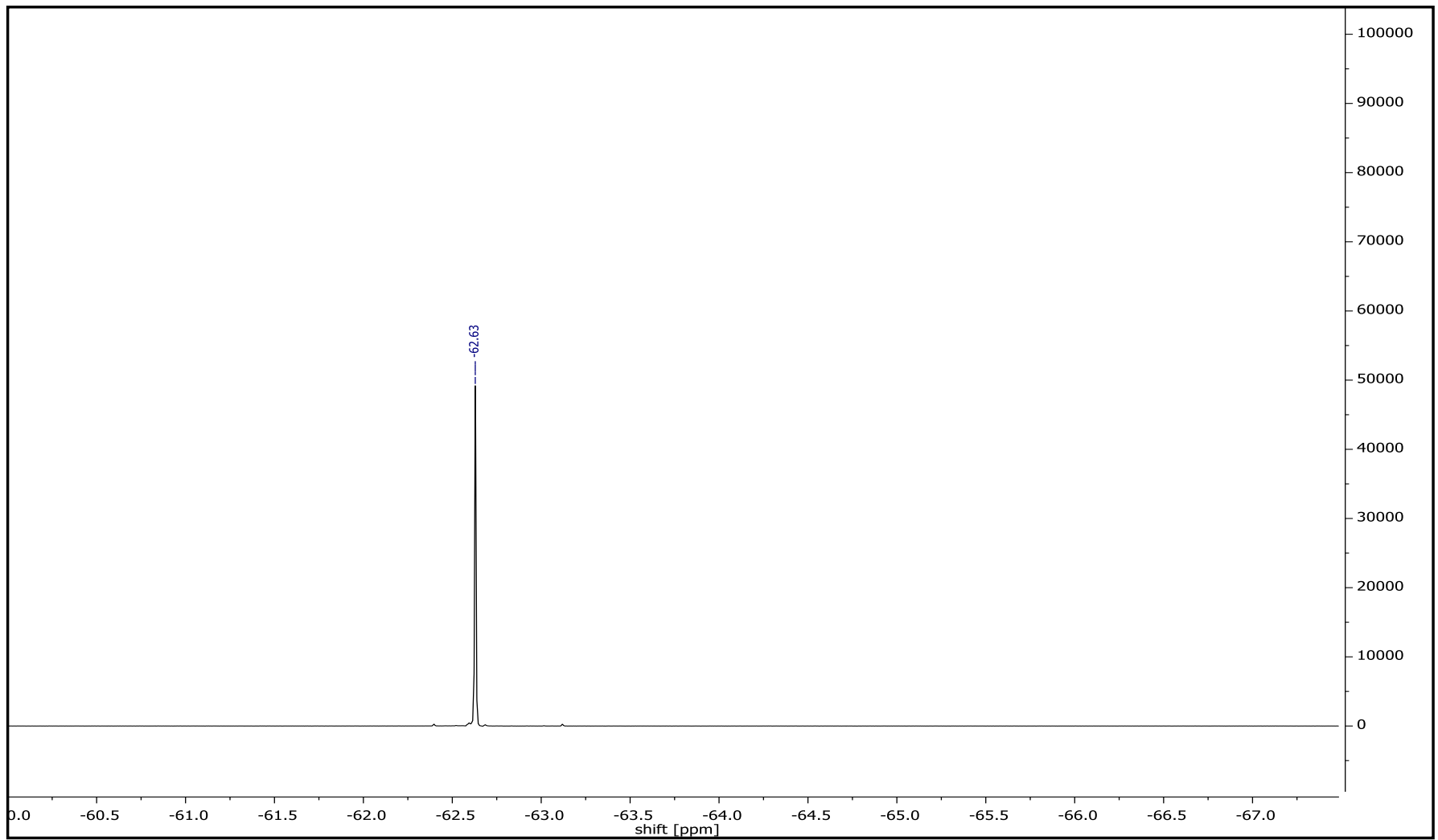


1-Methyl-3-(1-nitropropan-2-yl)benzene (19)

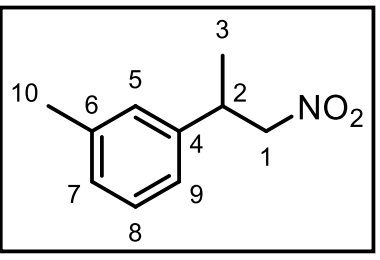

${ }^{1} \mathrm{H}-\mathrm{NMR}\left(400 \mathrm{MHz}, \mathrm{CDCl}_{3}\right)$

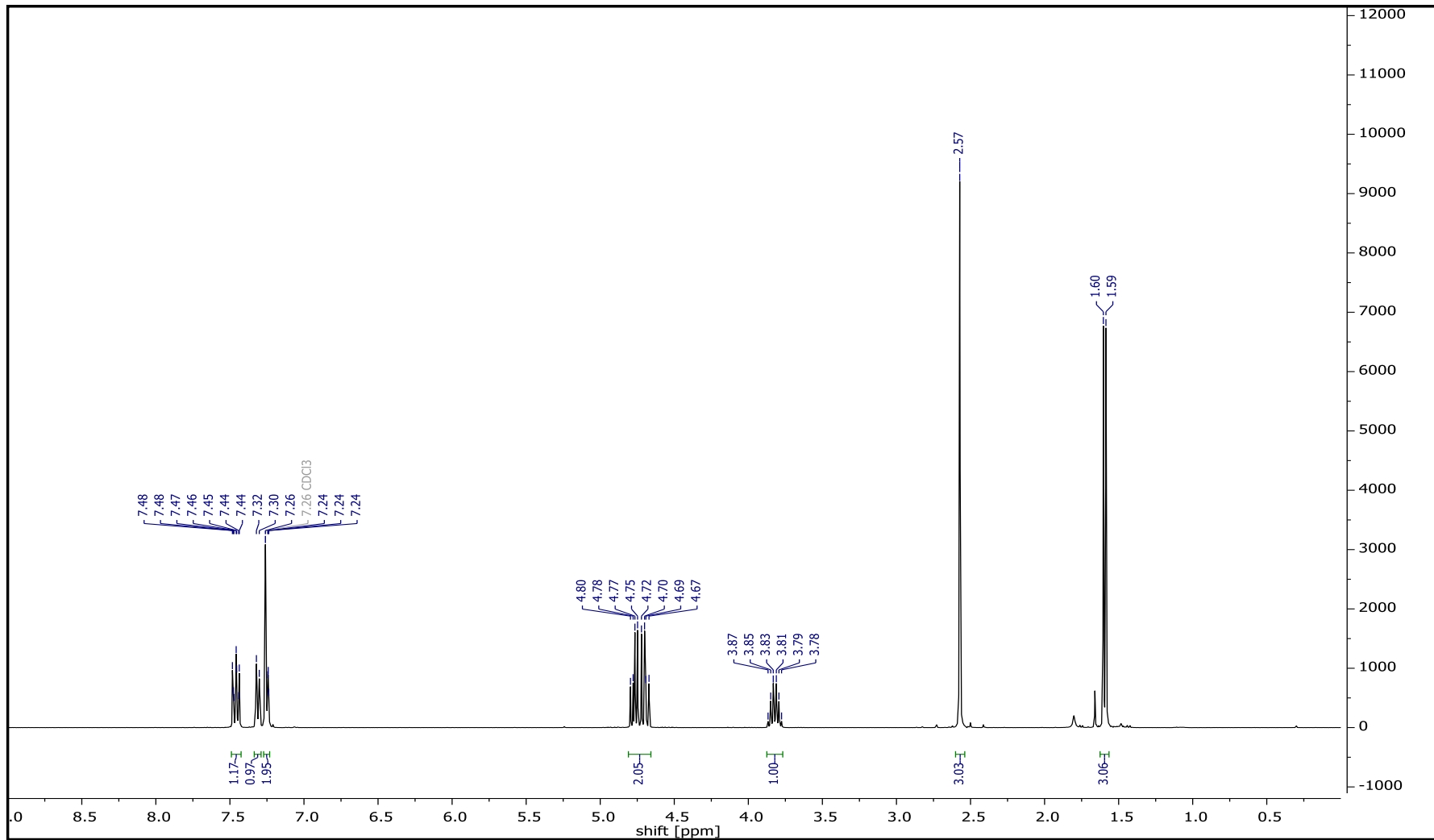

${ }^{13} \mathrm{C}-\mathrm{NMR}\left(101 \mathrm{MHz}, \mathrm{CDCl}_{3}\right)$

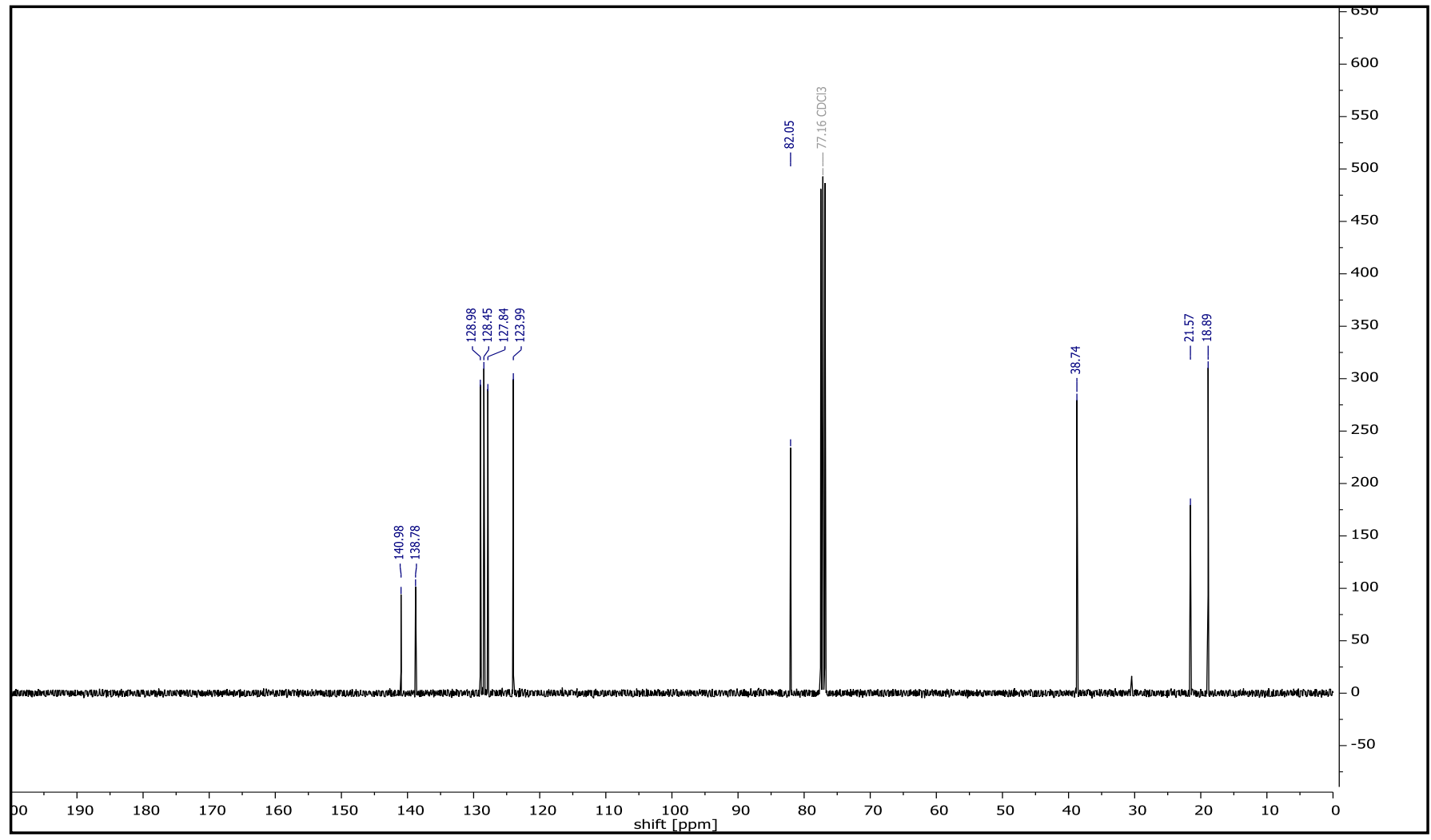


1-Chloro-3-(1-nitropropan-2-yl)benzene (20)

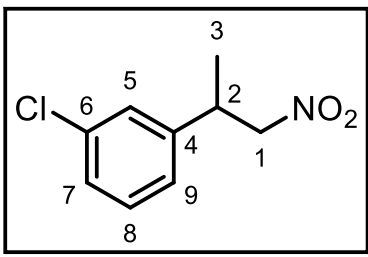

${ }^{1} \mathrm{H}-\mathrm{NMR}\left(400 \mathrm{MHz}, \mathrm{CDCl}_{3}\right)$

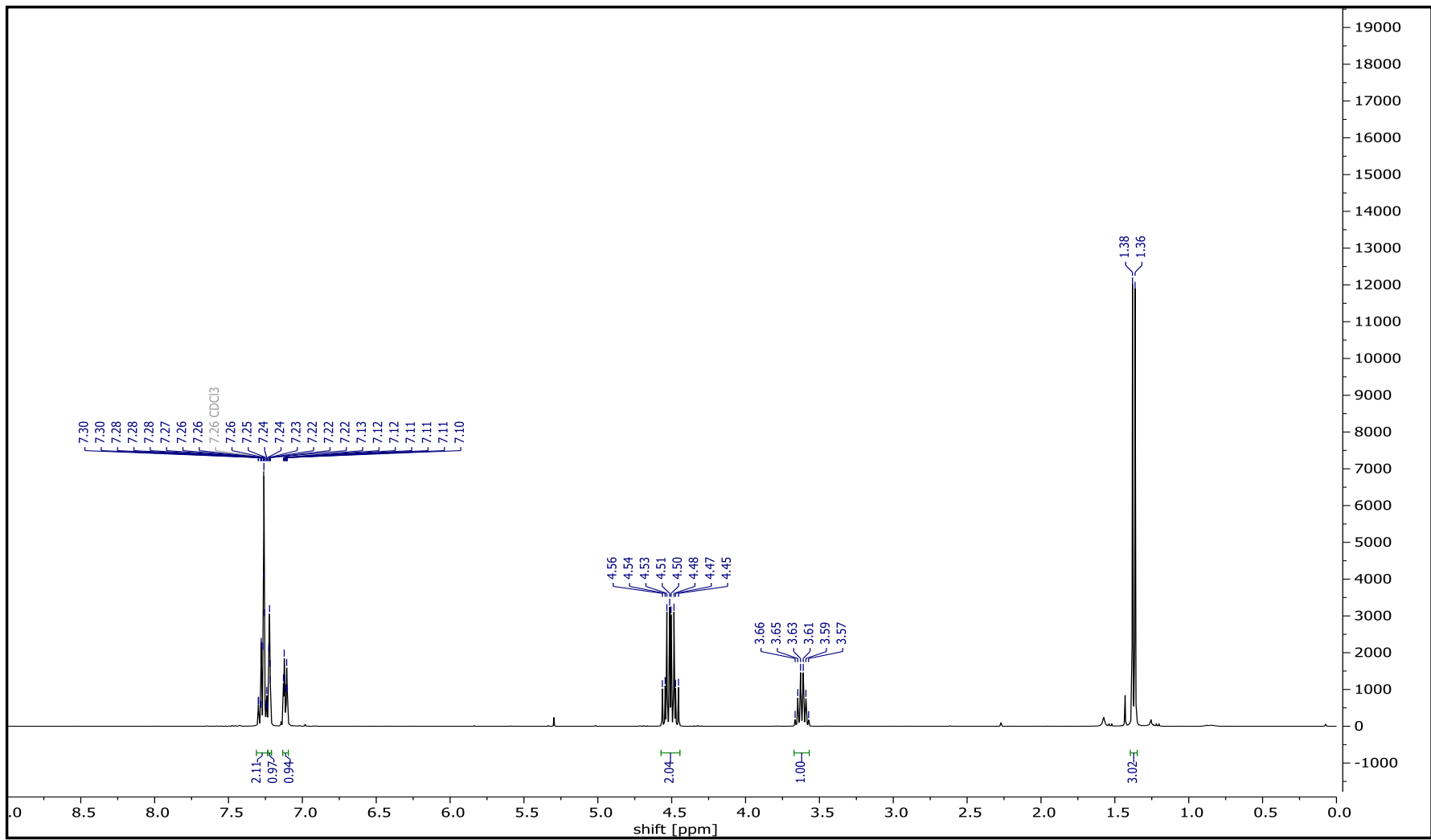

${ }^{13} \mathrm{C}-\mathrm{NMR}\left(101 \mathrm{MHz}, \mathrm{CDCl}_{3}\right)$

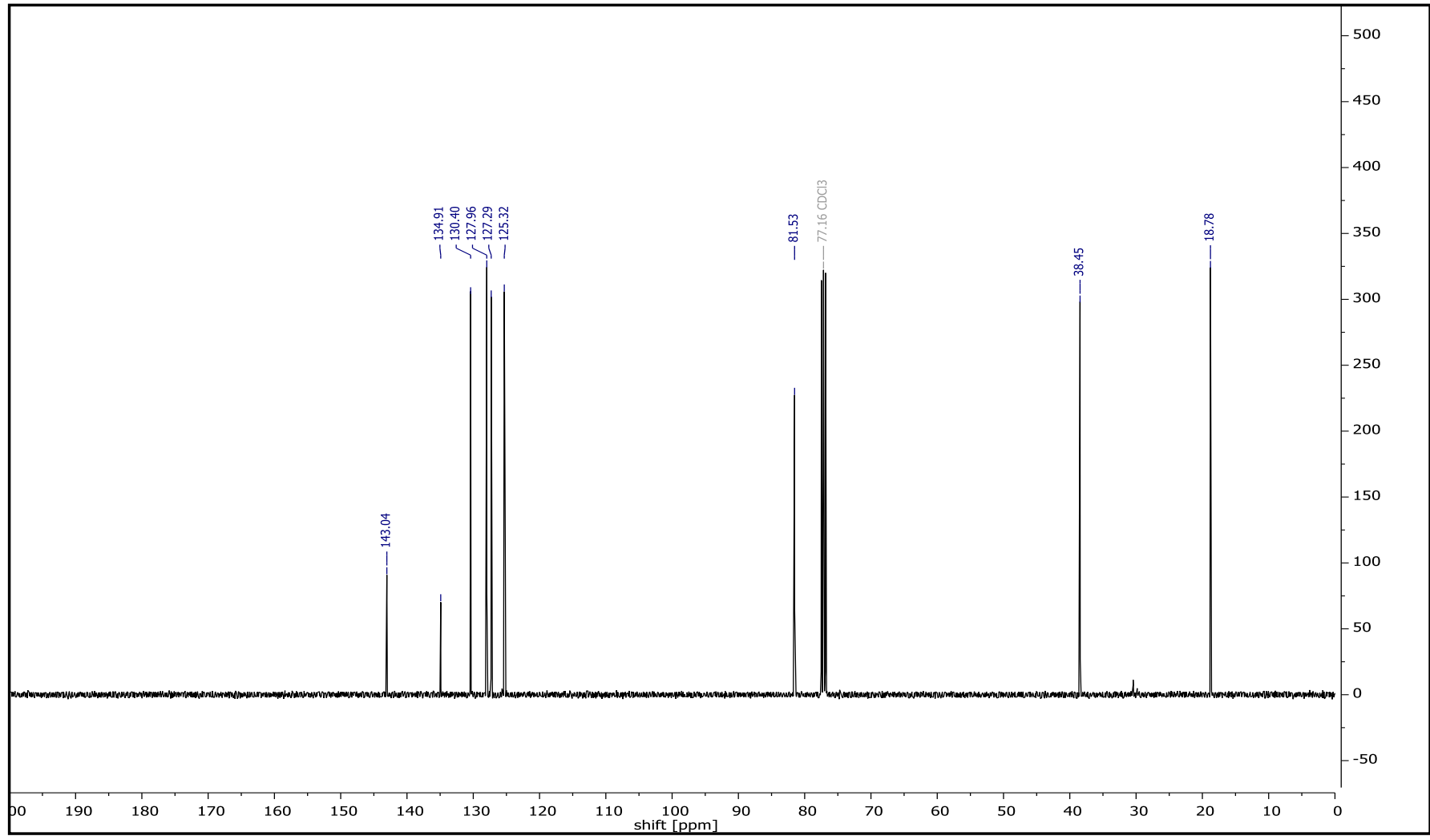




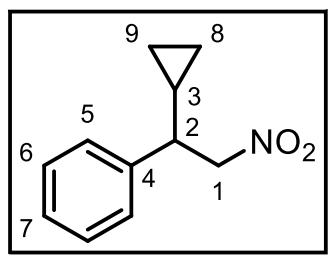

${ }^{1} \mathrm{H}-\mathrm{NMR}\left(500 \mathrm{MHz}, \mathrm{CDCl}_{3}\right)$

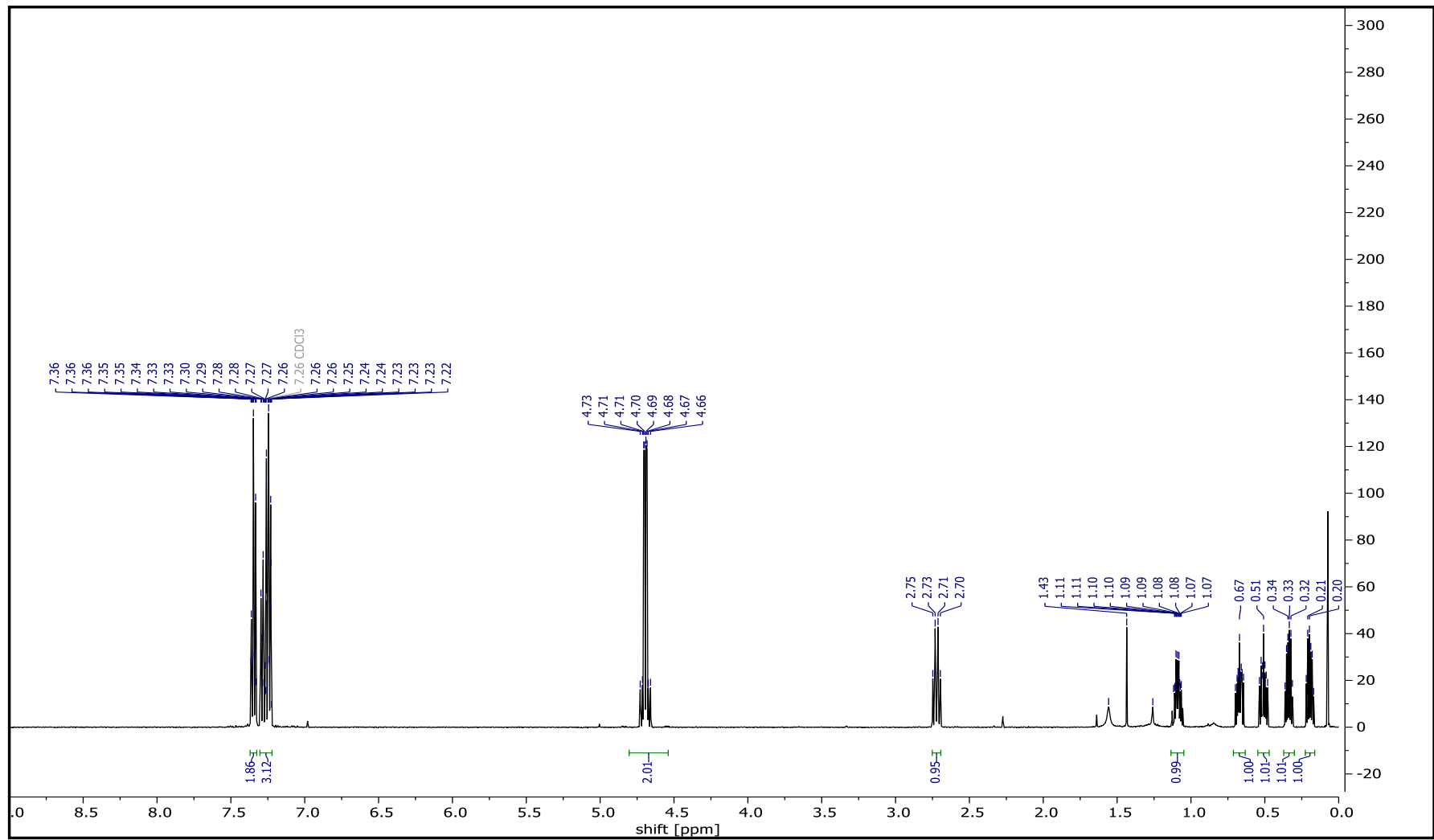

${ }^{13} \mathrm{C}-\mathrm{NMR}\left(126 \mathrm{MHz}_{\mathrm{CDCl}}\right)$

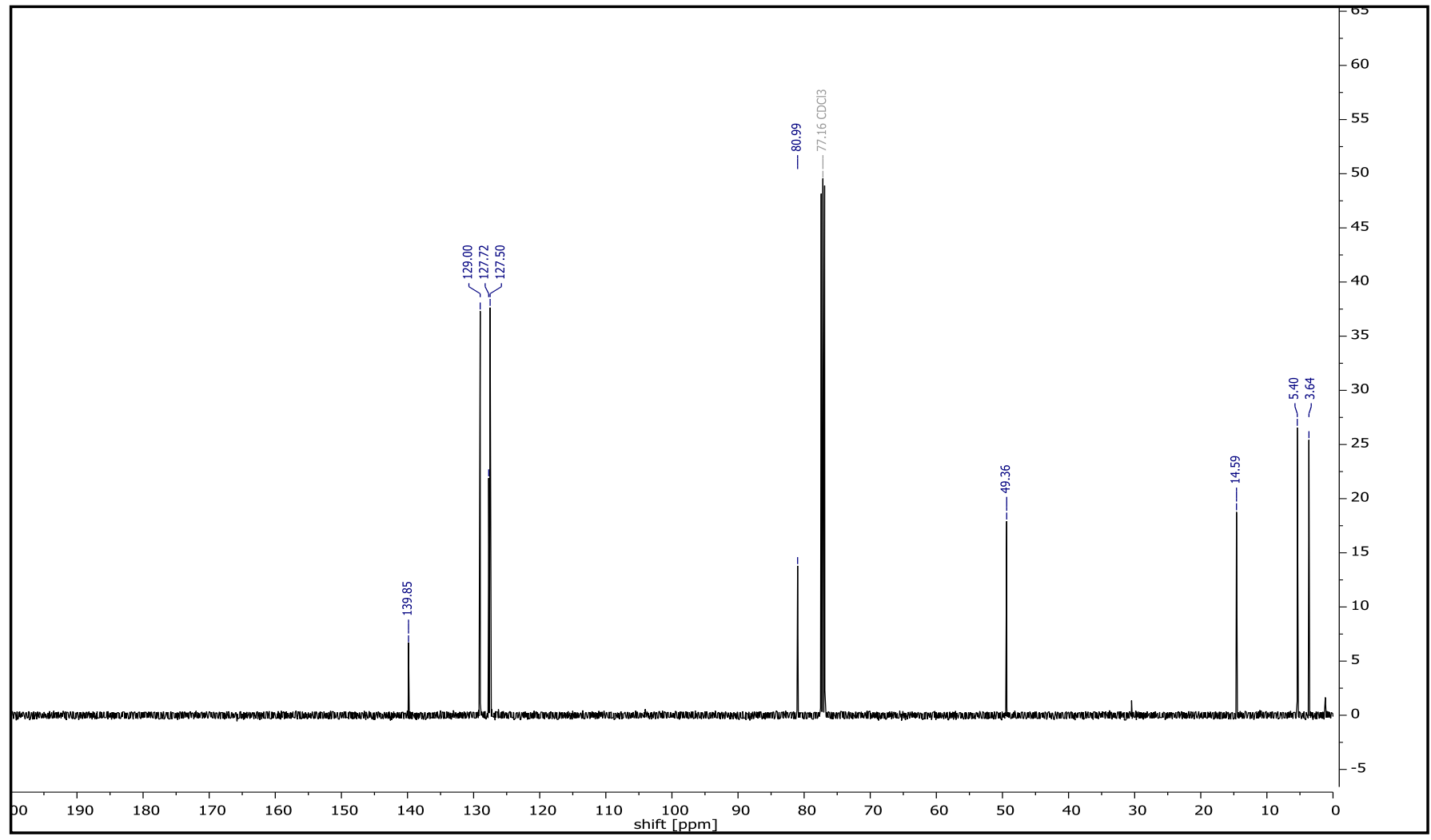


(S)-2-Isothiocyanato-N,N,3-trimethylbutanamide (S5)

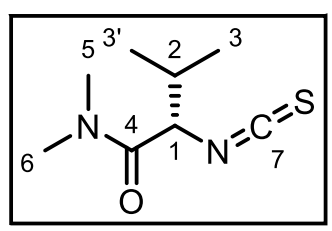

${ }^{1} \mathrm{H}-\mathrm{NMR}\left(500 \mathrm{MHz}^{\mathrm{CDCl}}{ }_{3}\right)$

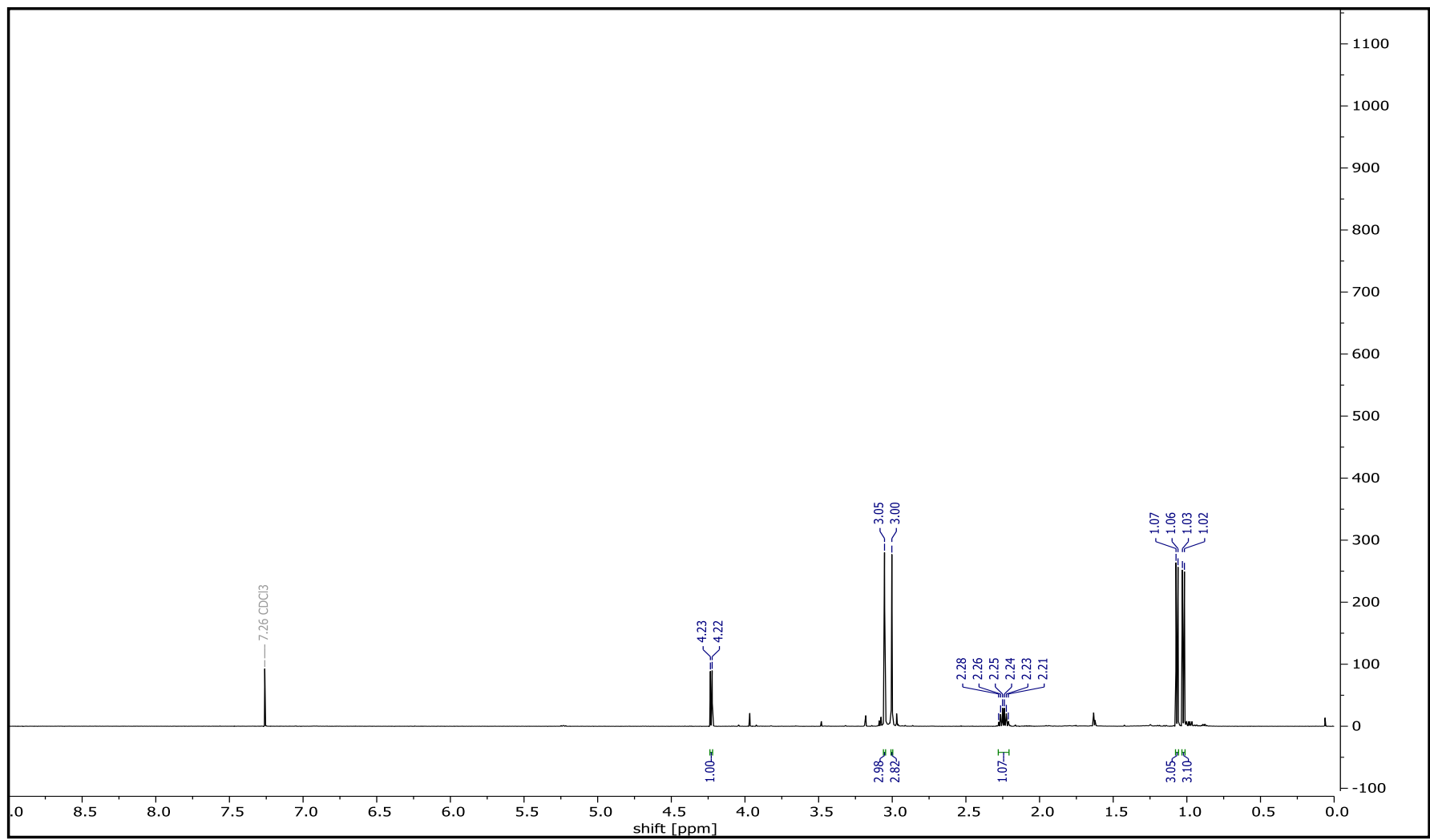

${ }^{13} \mathrm{C}-\mathrm{NMR}\left(300 \mathrm{MHz} \mathrm{CDCl}_{3}\right)$

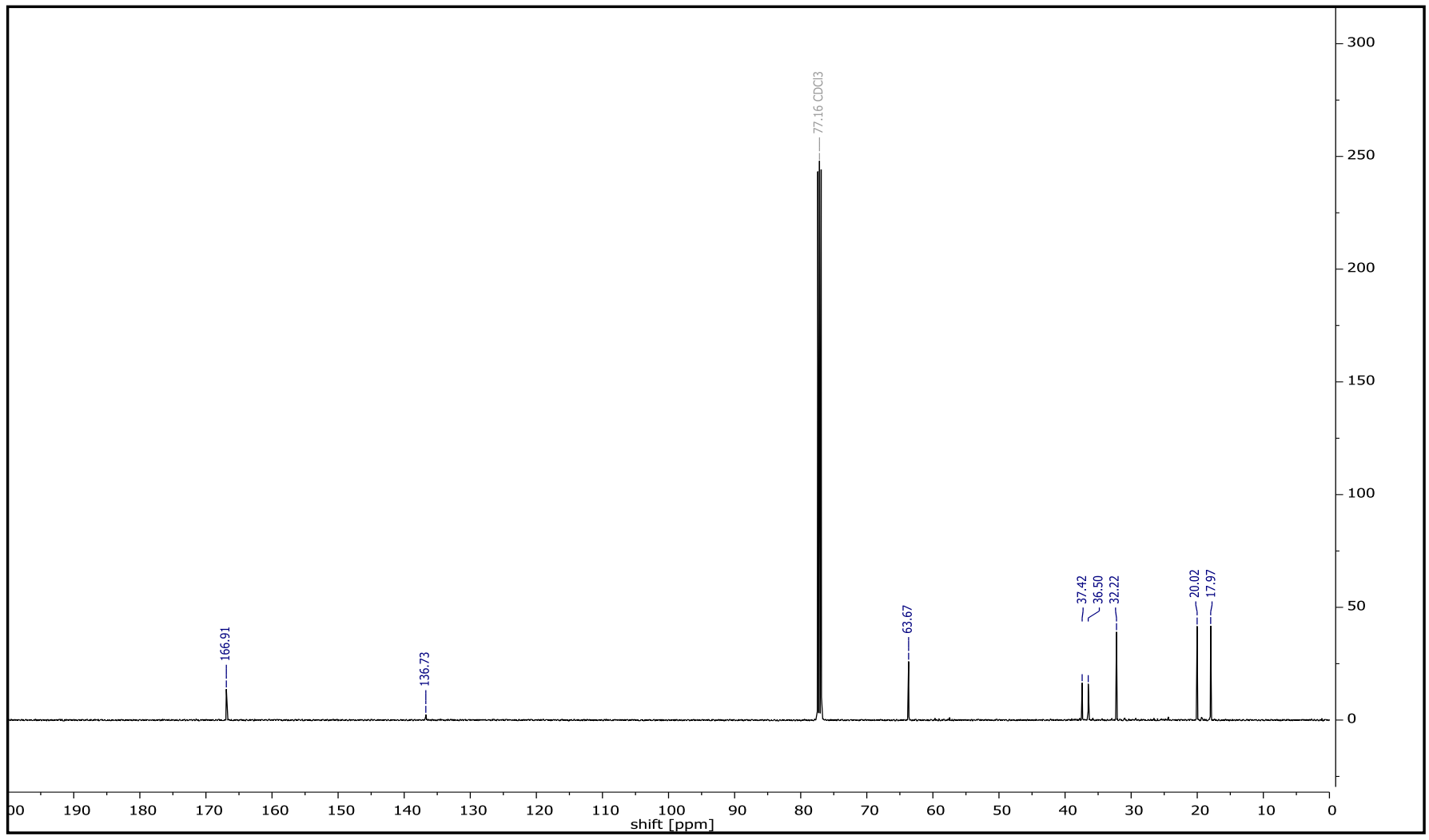


(S)-2-(3-((1R,2R)-2-(2,5-Dimethyl-1H-pyrrol-1-yl)cyclohexyl) thioureido)-N,N,3-trimethylbutanamide (A)

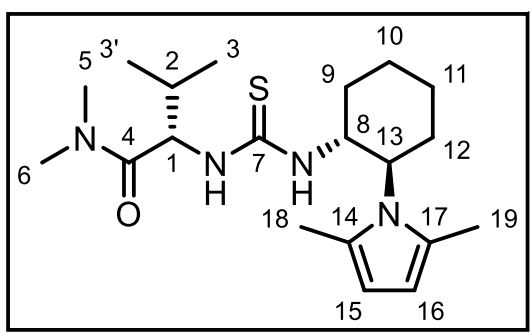

${ }^{1} \mathrm{H}-\mathrm{NMR}\left(500 \mathrm{MHz}, \mathrm{CDCl}_{3}\right)$

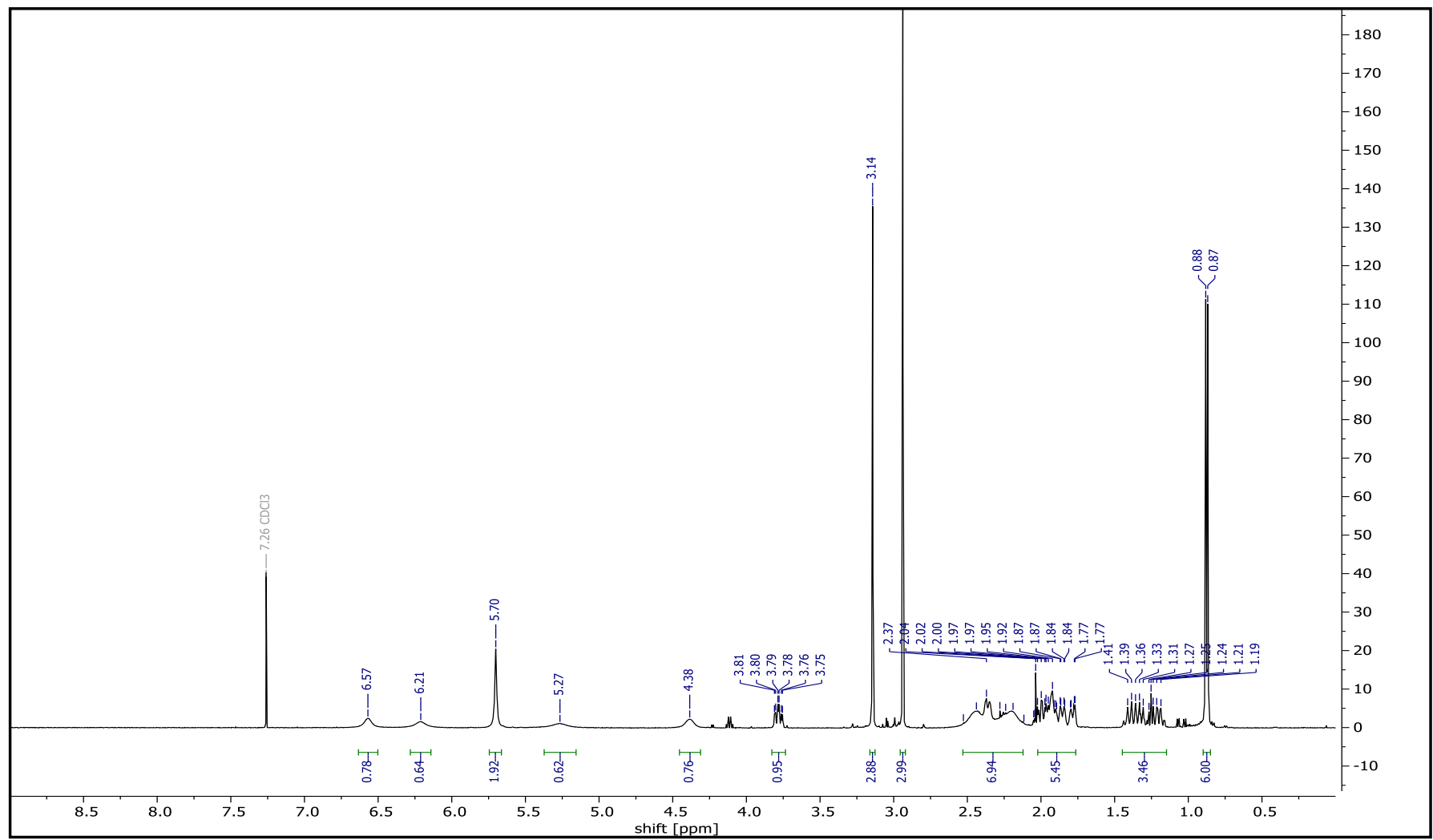

${ }^{13} \mathrm{C}-\mathrm{NMR}\left(126 \mathrm{MHz}, \mathrm{CDCl}_{3}\right)$

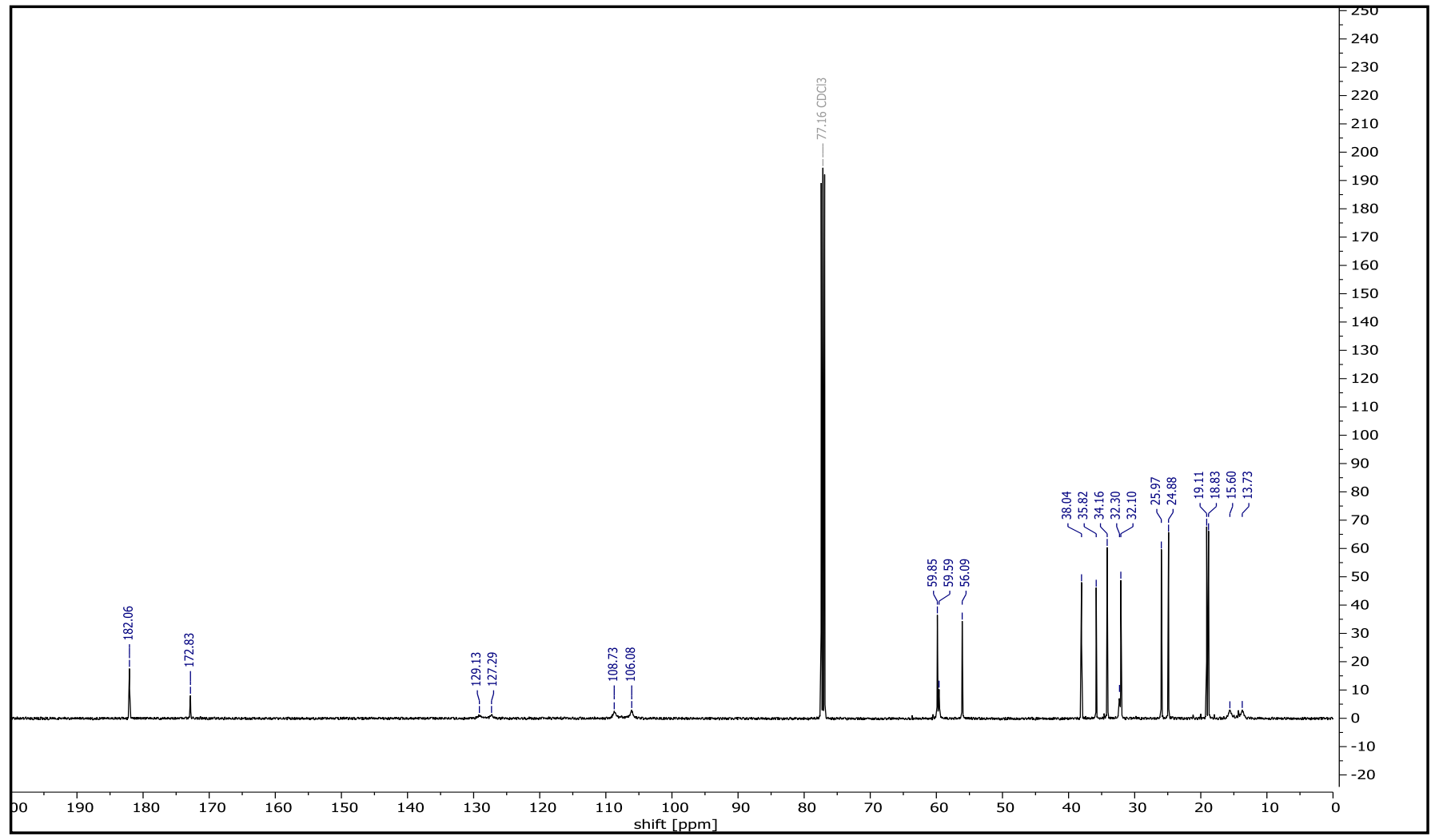


(S)-2-(3-((1R,2R)-2-(2,5-Dimethyl-1H-pyrrol-1-yl) cyclohexyl)thioureido)- $N, N, 3-$ trimethylbutanamide (B)

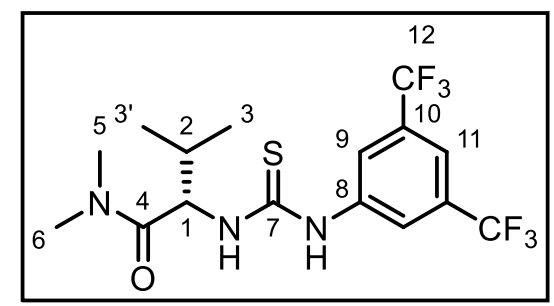

${ }^{1} \mathrm{H}-\mathrm{NMR}\left(600 \mathrm{MHz}, \mathrm{CDCl}_{3}\right)$

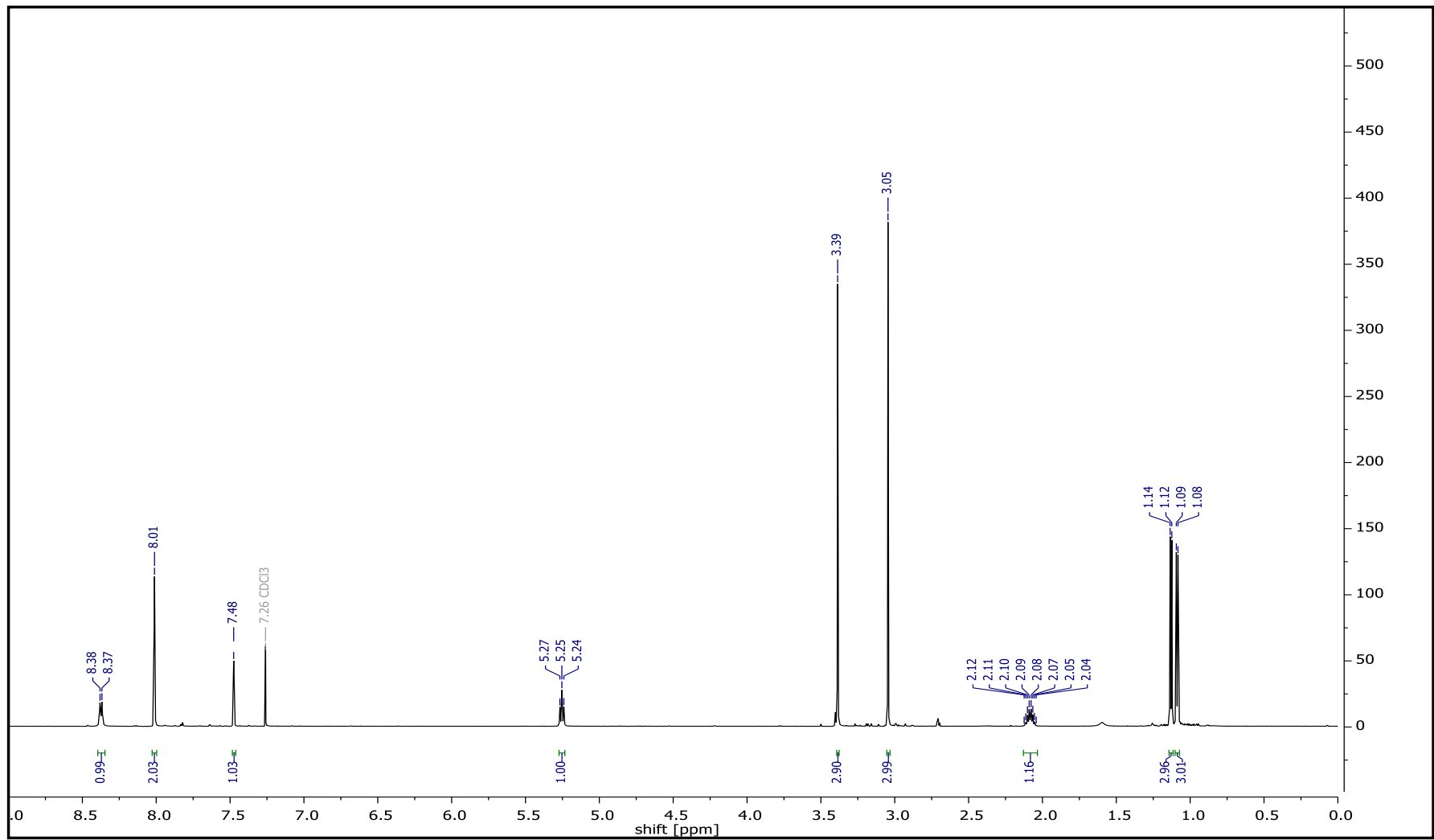

${ }^{13} \mathrm{C}-\mathrm{NMR}\left(151 \mathrm{MHz}, \mathrm{CDCl}_{3}\right)$

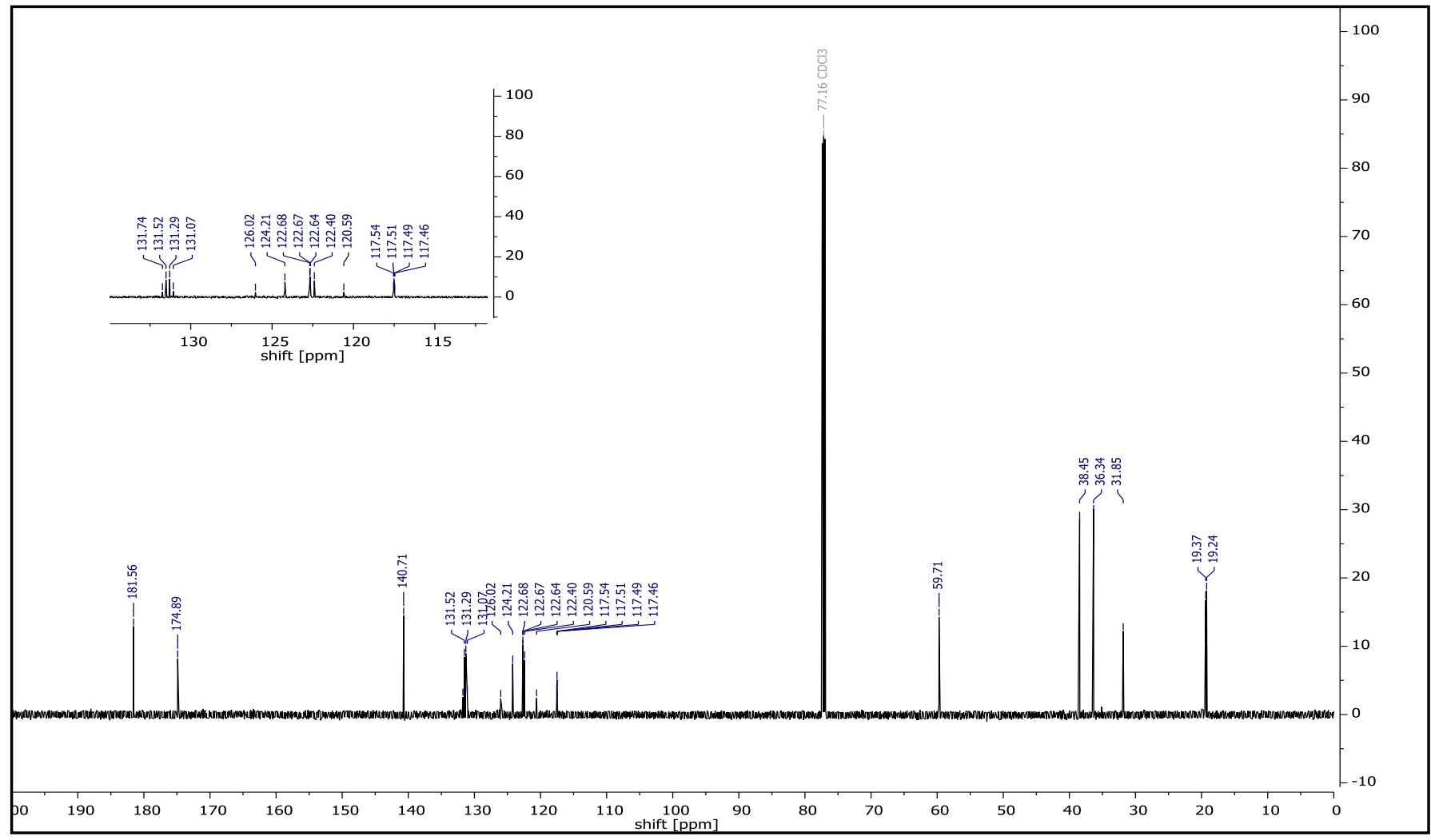


(S)-2-(3-((1R,2R)-2-(2,5-Dimethyl-1H-pyrrol-1-yl) cyclohexyl)thioureido)-N,N,3-trimethylbutanamide (B)

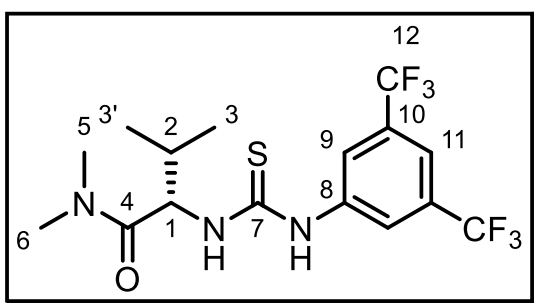

${ }^{19}$ F-NMR $\left(564 \mathrm{MHz}, \mathrm{CDCl}_{3}\right)$

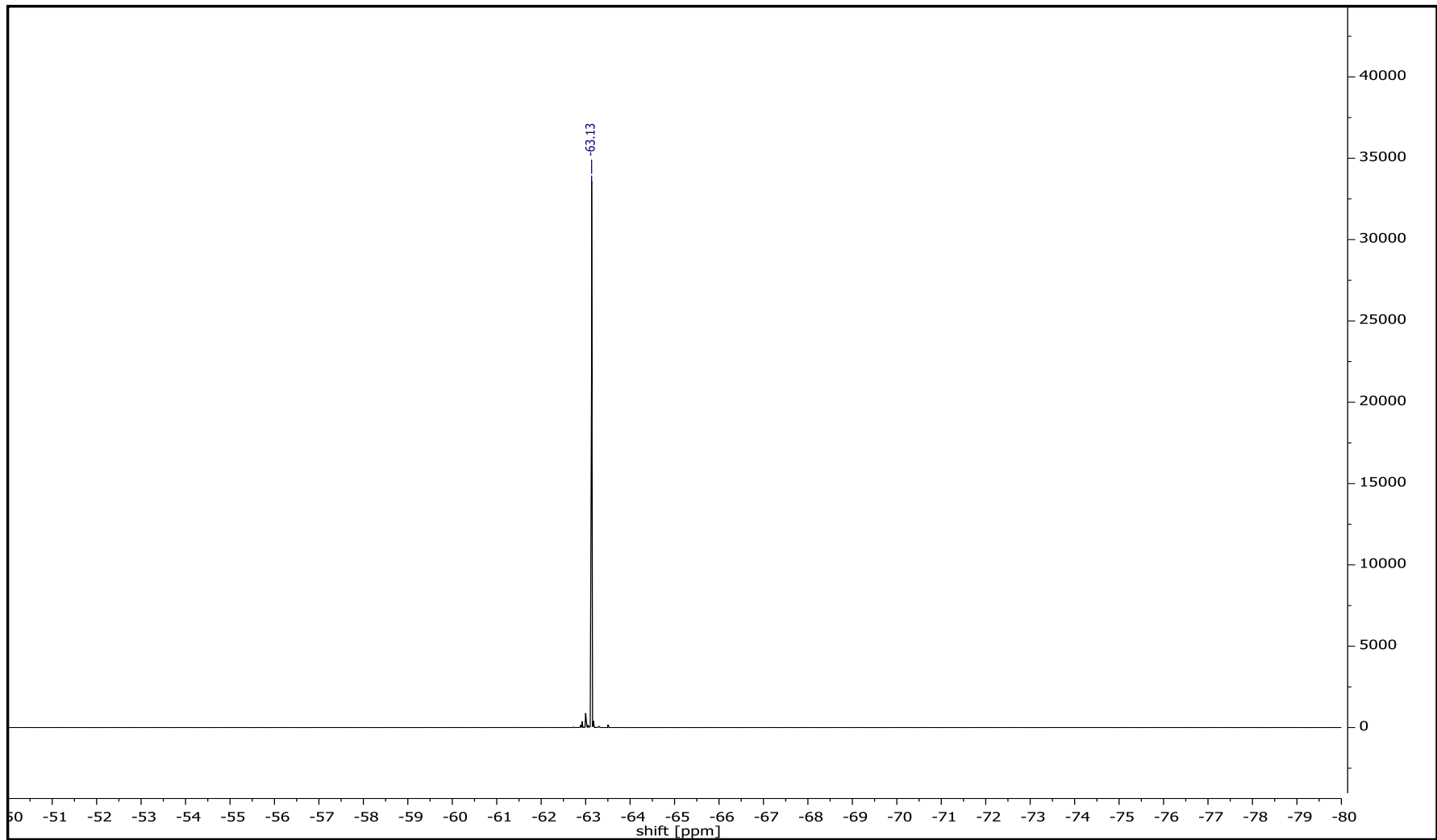




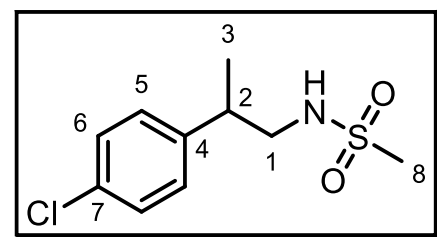

${ }^{1} \mathrm{H}-\mathrm{NMR}\left(300 \mathrm{MHz}, \mathrm{CDCl}_{3}\right)$

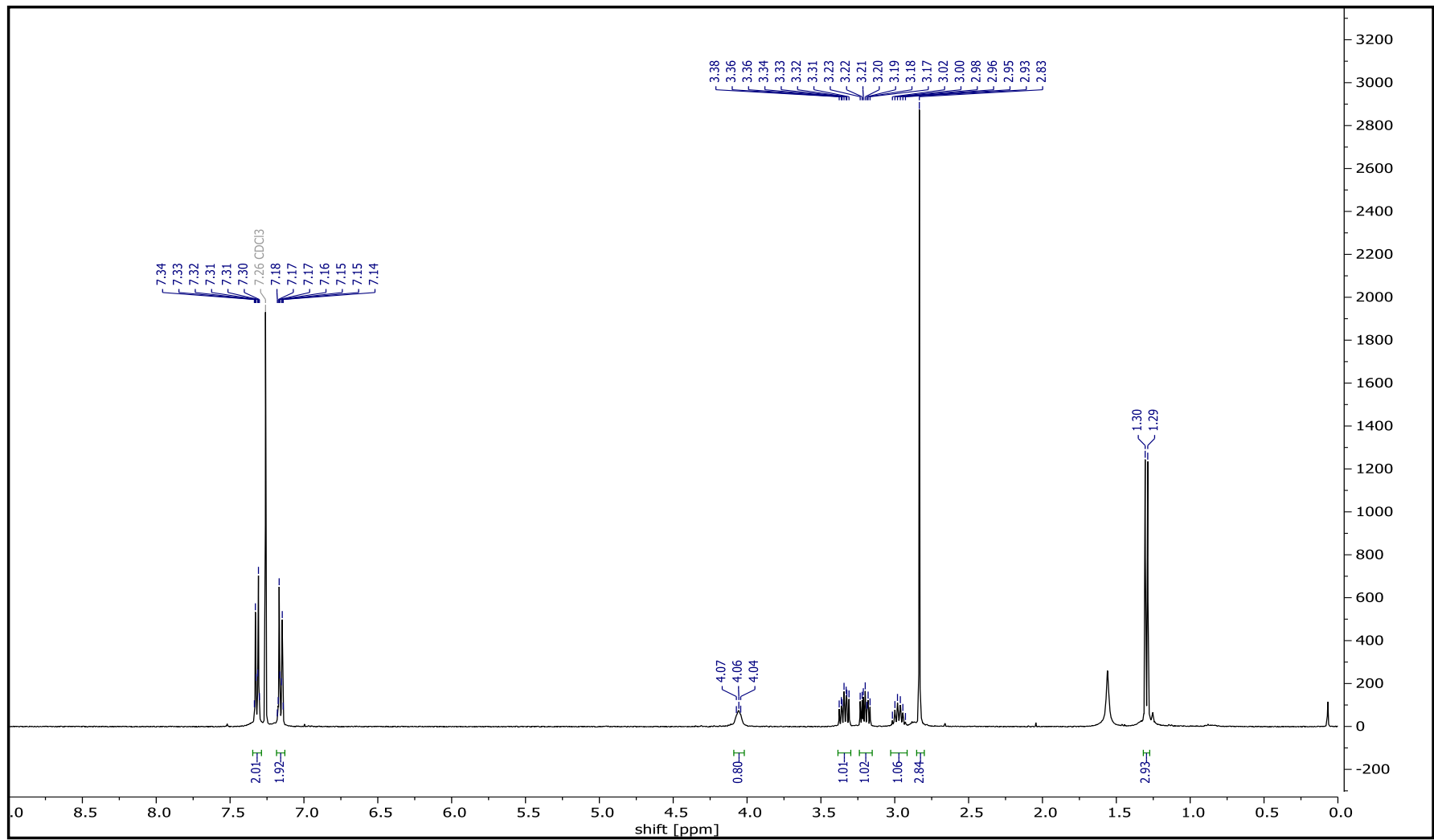

${ }^{13} \mathrm{C}-\mathrm{NMR}\left(300 \mathrm{MHz}, \mathrm{CDCl}_{3}\right)$

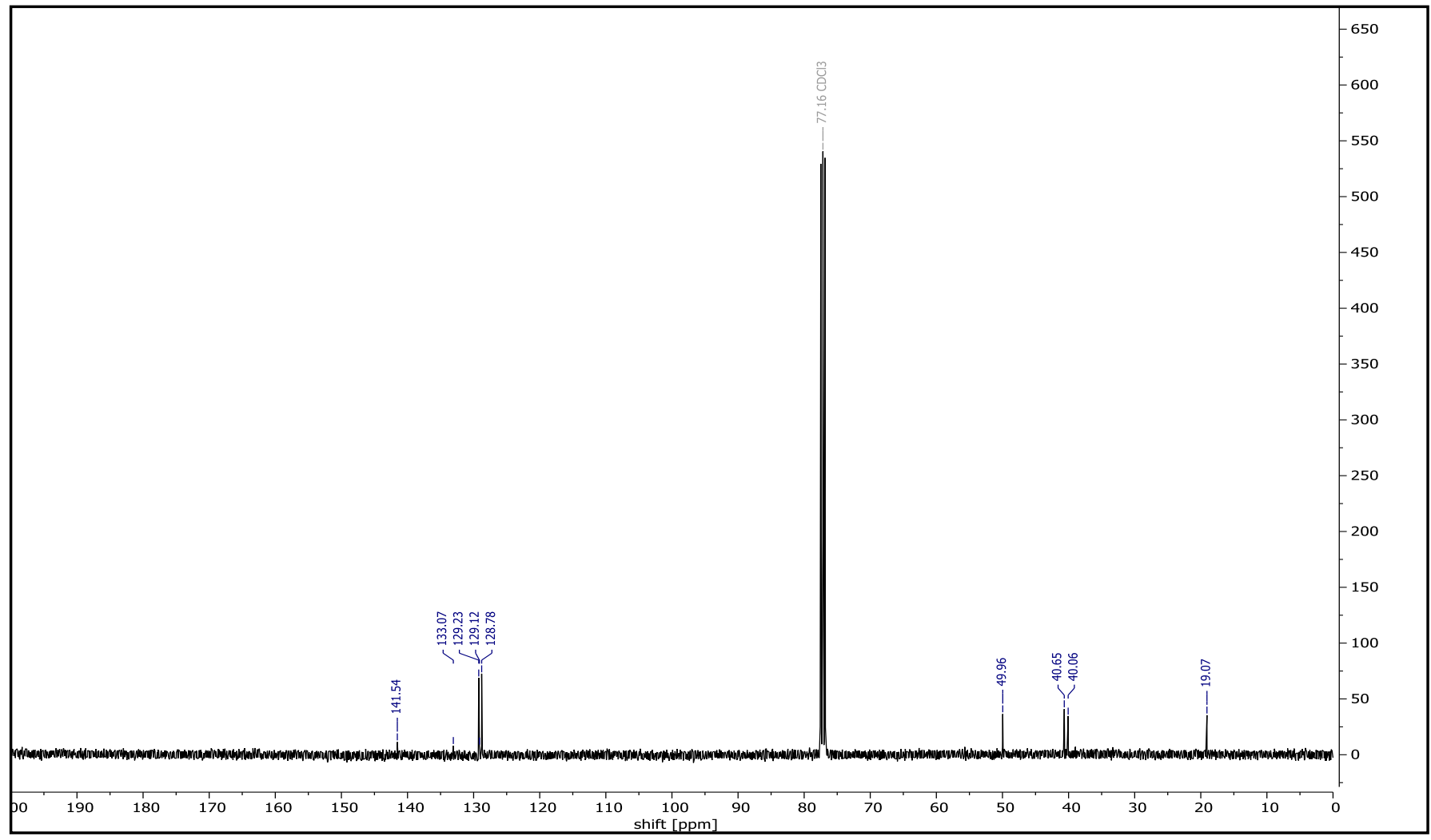


8-Chloro-1-methyl-2,3,4,5-tetrahydro-1H-benzo[d]azepine hydrochloric acid (24)

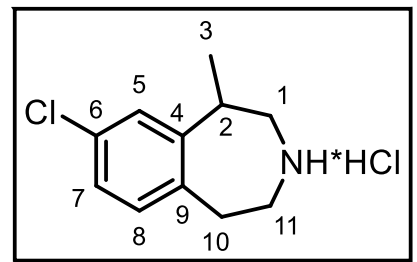

${ }^{1} \mathrm{H}-\mathrm{NMR}\left(600 \mathrm{MHz}, \mathrm{CDCl}_{3}\right)$

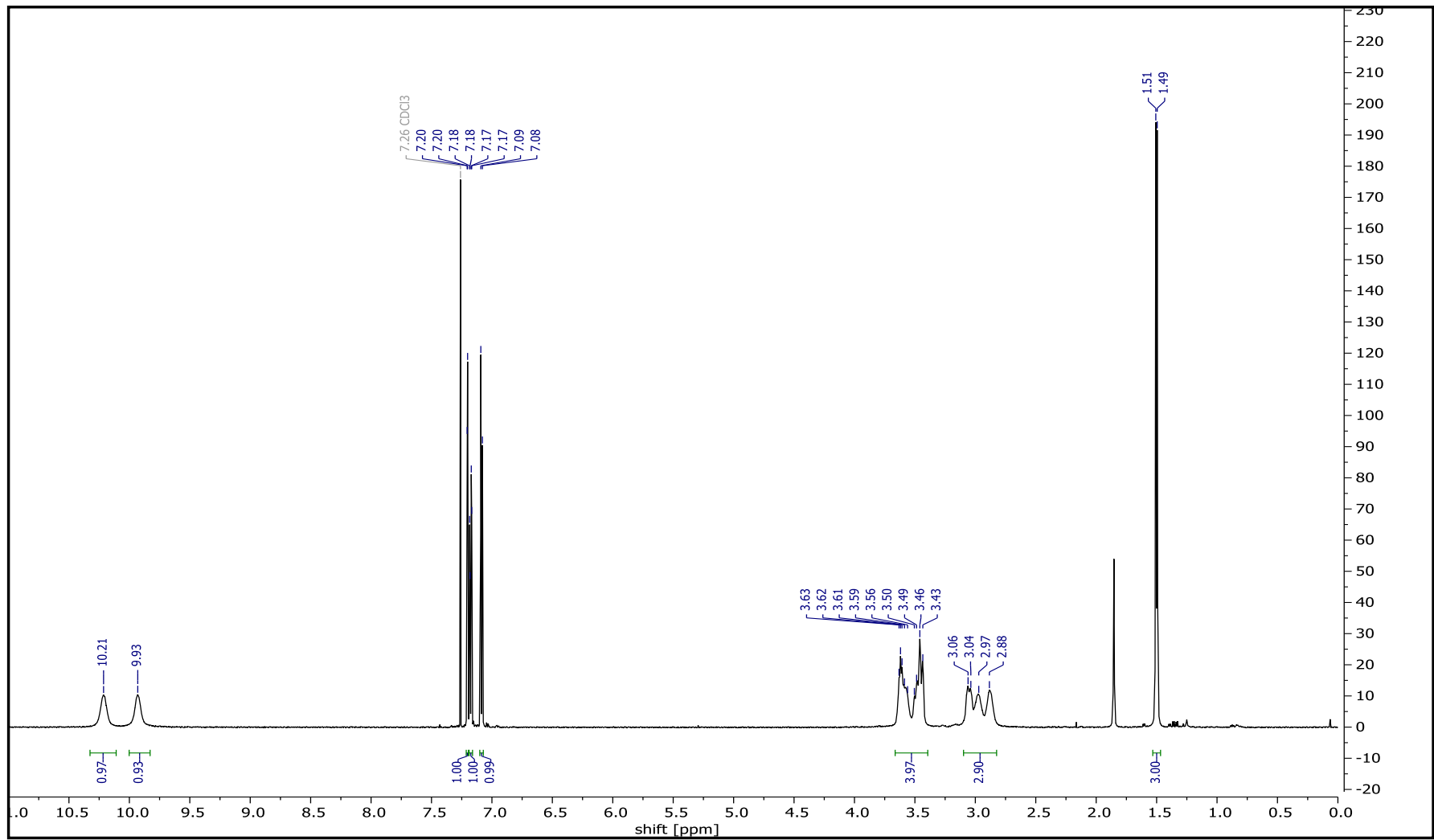

${ }^{13} \mathrm{C}-\mathrm{NMR}\left(151 \mathrm{MHz}, \mathrm{CDCl}_{3}\right)$

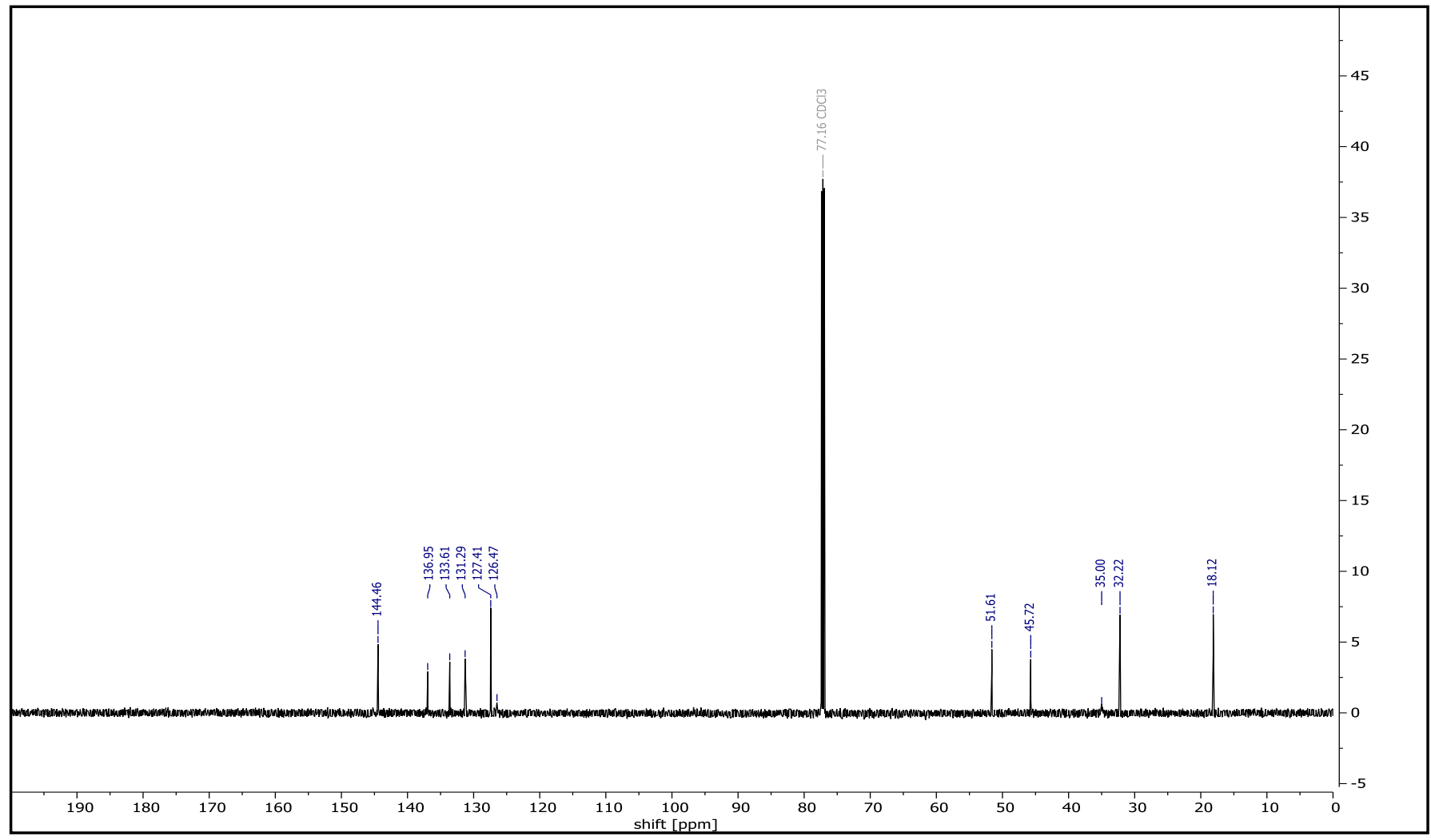

